\title{
Testing the Predictability of Stock Returns
}

\author{
Michel Fuksa \\ Thesis submitted to the \\ Faculty of Public Affairs and Management \\ in partial fulfilment of the requirements of the degree \\ Doctor of Philosophy in Management \\ Eric Sprott School of Business \\ Carleton University \\ Ottawa, Ontario
}

Copyright (C) Michel Fuksa 2002

September 17, 2002 
National Library

of Canada

Acquisitions and Bibliographic Services

395 Wellington Street Ottawa ON KIA ON4 Canada
Bibliothèque nationale

du Canada

Acquisitions et services bibliographiques

395. Jue Wellington

Ottawa ON KIA ON4

Canada
The author has granted a nonexclusive licence allowing the National Library of Canada to reproduce, loan, distribute or sell copies of this thesis in microform, paper or electronic formats.

The author retains ownership of the copyright in this thesis. Neither the thesis nor substantial extracts from it may be printed or otherwise reproduced without the author's permission.
L'auteur a accordé une licence non exclusive permettant à la Bibliothèque nationale du Canada de reproduire, prêter, distribuer ou vendre des copies de cette thèse sous la forme de microfiche/film, de reproduction sur papier ou sur format électronique.

L'auteur conserve la propriété du droit d'auteur qui protège cette thèse. Ni la thèse ni des extraits substantiels de celle-ci ne doivent être imprimés ou autrement reproduits sans son autorisation. 
The undersigned recommend to

The Faculty of Graduate Studies and Research acceptance of the thesis

\section{"TESTING THE PREDICTABILITY OF STOCK RETURNS"}

submitted by MICHEL FUKSA, B.Com., M.M.S.

in partial fulfillment of the requirements for the degree of Doctor of Philosophy, Management.

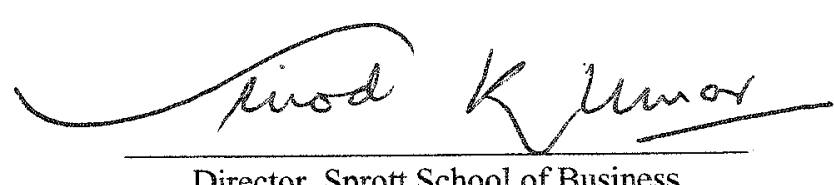

Director, Sprott School of Business
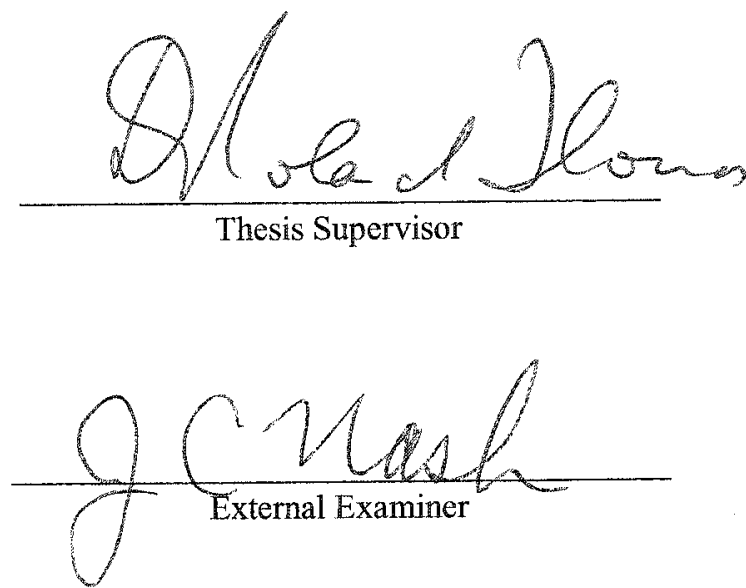

CARLETON UNIVERSITY

Tuesday, September 17, 2002 


\begin{abstract}
The predictability of stock prices is surely the oldest topic in financial research. Started early in the century, the empirical and theoretical quest for explaining the behaviour of financial markets continues today. During this time, many discoveries about the nature of stock prices and returns have been made, and different theories of behaviour have been proposed. These developments, in turn, have led to the creation of models for the prediction of returns.

While the literature has shown that there exists a certain level of predictability in stock returns, it is believed that the models that have been proposed to date lack explanatory power. The work proposes a new approach to forecasting stock returns based on emulating investor behaviour in processing information.

The work proposes that investors analyze information by weighing it according to its perceived accuracy. Investors give more importance to data they think is accurate, and discount information they perceive to be erroneous. The result of this analysis is an estimate of the condition, or "state", of the potential investments which balances available information according to its relevance.

This process can be compared to the concept of statistical signal filtering. The study uses the Kalman filter to emulate the investors' analytical process and implements several empirical models to test the predictability of stock returns. The results show that while it is difficult to predict the behaviour of returns, the principal cause lies in the models' inability to explain returns' volatility.
\end{abstract}




\section{Acknowledgement}

During the time of my studies I have received support and encouragement from many people. I would like to take the time to mention some of them.

First, I would like to thank very kindly my thesis supervisor, Professor Roland Thomas, for the many years he has devoted to supervising my research. He has proven to be an exceptional teacher, and has actively encouraged me to explore areas of statistics previously unknown to me, and by doing so, has helped to significantly broaden my horizons. I would also like to thank him for the generous support he has offered me during my stay at Carleton.

I would like to kindly thank Professor George Haines for encouraging me in my research and for the many valuable discussions he allowed me to have with him. His knowledge of many different fields has made him invaluable in providing new perspectives on many different topics, and has allowed me to formulate many ideas for future research.

I would like to also thank Professor Michael McIntyre for the many fruitful discussions and the many ideas he has provided for improving my research. His attention to detail has led me to greatly improve my work.

Finally, I would like to thank the School's Graduate Secretary, Ms. Jean Blair, for always keeping the required bureaucracy in check, and for the kind words of encouragement during the long years of study. 


\section{Contents}

1 Introduction 1

2 Evidence on Market Efficiency 4

2.1 Introduction ...................... 4

2.2 Theory of Market Efficiency ............. . . . . 4

2.2 .1 Bachelier, $1900 \ldots \ldots \ldots \ldots \ldots \ldots$

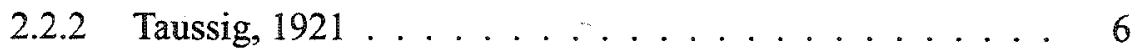

2.2 .3 Working, $1958 \ldots \ldots \ldots \ldots$

2.2 .4 Modern Developments . . . . . . . . . . . . . . 9

2.3 Distributional Properties of Returns . . . . . . . . . . . . . 11

2.4 Volatility Research . . . . . . . . . . . . . . . . . 19

2.5 Investor Behaviour . . . . . . . . . . . . 28

3 Models of Returns 36

3.1 Regression Models . . . . . . . . . . . . . . . . . . . 36

3.2 Time Series Models . . . . . . . . . . . . . . . . . . . . . . . . . . . . . . . . . 54

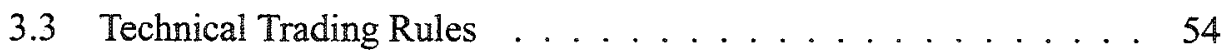

3.4 Conclusion . . . . . . . . . . . . . . . 66

4 A New Approach to Forecasting Stock Returns 68

4.1 Introduction . . . . . . . . . . . . . . 68

4.2 A Model of the Investor . . . . . . . . . . . . . . . . . 69

4.3 A Univariate Model of Stock Returns . . . . . . . . . . . . . 77

4.3.1 Measurement Equation . . . . . . . . . . . 79

4.3.2 Firm Transition Equation . . . . . . . . . . . . . . 80

4.4 The Multivariate Model . . . . . . . . . . . . . . . . . 81

4.5 The proposed models and the literature . . . . . . . . . 88

4.6 Prediction and Testing . . . . . . . . . . . . . . 89 
4.6.1 The Data . . . . . . . . . . . . . . . 89

4.6 .2 Methodology . . . . . . . . . . . . . . 90 90

4.6.3 Prediction: Univariate Model . . . . . . . . . . . . . . . 92

4.6.4 Prediction: Multivariate Model . . . . . . . . . . . . 93

4.6.5 Prediction Windows ... . . . . . . . . . . . . 94

4.6 .6 Model Testing . . . . . . . . . . . . . . 94

5 Results $\quad 97$

5.1 Introduction . . . . . . . . . . . . . . . . 97

5.2 The Data . . . . . . . . . . . . . . . . . . . . . . 98

5.3 The Estimation Method . . . . . . . . . . . . . . . . . 98

5.4 Estimation Results - Univariate Model . . . . . . . . . . . 100

5.4.1 Variance Assumptions ... . . . . . . . . . . . 101

5.4.2 Non-fundamental model component: daily effects . . . . 101

5.4.3 Fundamental model component: damped linear trend . . . 103

5.4.4 Non-fundamental model component: ARMA sub-model . 108

5.5 Estimation Results - Multivariate Model . . . . . . . . . . 108

5.6 Prediction Results: Univariate Model . . . . . . . . . . . . . . . 109

5.7 Prediction Results: Multivariate Model . . . . . . . . . . . . 110

5.7.1 Benchmark Model Comparisons . . . . . . . . . . 114

5.7.2 Simple time series benchmark models . . . . . . . . 114

5.7.3 GARCH model benchmarks . . . . . . . . . . 116

5.8 Sensitivity analysis . . . . . . . . . . . . . . . . 118

5.9 Further Sensitivity Analysis . . . . . . . . . . . . . 121

6 Time Varying Parameter Model 122

6.1 Introduction . . . . . . . . . . . . . . . . . . . . . . . . .

6.2 Variance Assumptions . . . . . . . . . . . . . . . 123

6.3 Estimation and Results . . . . . . . . . . . . . . . 123

6.4 An "average" model . . . . . . . . . . . . . . 126

$\begin{array}{lll}7 \text { Conclusion } & 128\end{array}$

$\begin{array}{ll}\text { A Data } & 135\end{array}$

B Estimation Algorithm 139

B.1 Introduction . . . . . . . . . . . . . . . . . 139

B.2 The Method . . . . . . . . . . . . . . . . 140 
B.3 The Algorithm . . . . . . . . . . . . . . . . . . . 144

B.3.1 Kalman propagation step . . . . . . . . . . . . . . . 144

B.3.2 Score equations (propagation step) . . . . . . . . 145

B.3.3 Conditional information matrix equations (propagation step) 145

B.3.4 Kalman updating step . . . . . . . . . . . . 145

B.3.5 Score equations (updating step) . . . . . . . . 146

B.3.6 Conditional information matrix equations (updating step) . 146

C Univariate Model 148

C.1 The Fundamental Component . . . . . . . . . . . . . . . . 148

C.2 The Daily Component . . . . . . . . . . . . . . . . . . 149

C.3 Non-fundamental Component . . . . . . . . . . . . . 149

C.4 The Complete Model . . . . . . . . . . . . . . . 150

D Univariate Model Results

E Multivariate Model 174

E.1 Multivariate Model: Market Submodel Estimate . . . . . . . . . . 174

E.2 Multivariate Model: Parameter Estimates . . . . . . . . . . . 179

$\begin{array}{ll}\mathbb{F} \text { Sensitivity Analysis } & 201\end{array}$

G Summary Tables $\quad 208$

H Time Varying Parameter Univariate Models 219 


\section{List of Tables}

2.1 Central moments of wheat prices (Kendall [Kendall, 1953]) . . . . 12

2.2 Engle, Lilien and Robins: ARCH-M models [Engle et al., 1987] . 35

2.3 French et al. excess return regressions on ARMA predicted volatilities [French et al., 1987] . . . . . . . . . . . . . 35

2.4 French et al. monthly excess return GARCH-M model results [French et al., 1987] . . . . . . . . . . . 35

3.1 Chan, Kacerski and Lakonishok [Chan et al., 1998] mimicking portfolio return statistics. . . . . . . . . . . . . . . . 37

3.2 Rozeff's [Rozeff, 1974] regression results . . . . . . . . . . . . . 39

3.3 Profitability of the model selection criteria based trading rules under No and High cost scenarios ([Pesaran and Timmerman, 1995] p. 1220) . . . . . . . . . . . . . . . 45

3.4 Conrad and Kaul estimates for the model: $R_{t}=\alpha+\beta_{1} \mathbb{E}_{t-1}\left(R_{t}\right)+$ $\beta_{2} R F_{t-1}+\beta_{3} E W M R_{t-1} \ldots \ldots \ldots \ldots \ldots$

3.5 Alexander's [Alexander, 1961] Filter Rule Profits . . . . . . . 56

3.6 Summary of DIIA average for 1897-1986 . . . . . . . . . . . 59

3.7 Trading rule results from Brock, Lakonishok and LeBaron [Brock et al., 1992] 60

3.8 Simulation results: average "Buy-Sell" retums for the VMA, FMA and TRB trading rules . . . . . . . . . . . 60

5.1 Dividend and split adjusted prices and returns for 10 selected stocks. 99

5.2 Results of estimation and prediction using Harvey's time varying daily effects model. . . . . . . . . . . . . . . . . . . . . 104

5.3 Results of estimation and prediction using dummy variables model. 105

5.4 Prediction results for selected univariate models. . . . . . . . . . 111

5.5 Prediction results for selected multivariate models. . . . . . . . 113

5.6 Random Walk model prediction results. . . . . . . . . . . 115

5.7 AR(1) model prediction results. . . . . . . . . . 117 
5.8 Prediction results stemming from the GARCH and GJR models for all stocks in the sample. . . . . . . . . . . . . . . 119

A.1 Selected Dow Jones Industrial Average stock components. . . . 137

A.2 Selected stock indices used in the study. . . . . . . . . . 138

A.3 Selected exchange rate data used in the study. . . . . . . . 138

D.1 Parameter estimates for the univariate model. Stock BA . . . . 154

D.2 Parameter estimates for the univariate model. Stock C . . . . . 155

D.3 Parameter estimates for the univariate model. Stock GE . . . . 156

D.4 Parameter estimates for the univariate model. Stock IBM . . . . 157

D.5 Parameter estimates for the univariate model. Stock INTC . . . 158

D.6 Parameter estimates for the univariate model. Stock JPM . . . . 159

D.7 Parameter estimates for the univariate model. Stock MMM . . . 160

D.8 Parameter estimates for the univariate model. Stock MRK . . . 161

D.9 Parameter estimates for the univariate model. Stock MSFT . . . 162

D.10 Parameter estimates for the univariate model. Stock XOM ... 163

E.1 Multivariate model parameters. Stocks BA - INTC. . . . . 179

E.2 Multivariate model parameters. Stocks JPM - XOM. . . . . . . 180

F.1 Univariate model sensitivity analysis: parameter estimates for selected values of R. Stock:BA . . . . . . . . . . . . . 201

F.2 Univariate model sensitivity analysis: parameter estimates for selected values of R. Stock:C . . . . . . . . . . . . . . 202

F.3 Univariate model sensitivity analysis: parameter estimates for selected values of R. Stock:GE . . . . . . . . . . . . . . 202

F.4 Univariate model sensitivity analysis: parameter estimates for selected values of R. Stock:IBM _. . . . . . . . . . 203

F.5 Univariate model sensitivity analysis: parameter estimates for selected values of R. Stock:INTC . . . . . . . . . . . . 203

F.6 Univariate model sensitivity analysis: parameter estimates for selected values of R. Stock:JPM . . . . . . . . . . . . 204

F.7 Univariate model sensitivity analysis: parameter estimates for selected values of R. Stock:MMM . . . . . . . . . . . . . 204

F.8 Univariate model sensitivity analysis: parameter estimates for selected values of R. Stock:MRK . . . . . . . . . . . 205

F.9 Univariate model sensitivity analysis: parameter estimates for selected values of R. Stock:MSFT . . . . . . . . . 205 
F.10 Univariate model sensitivity analysis: parameter estimates for selected values of R. Stock:XOM . . . . . . . . . . . . . 206

F.11 Univariate model sensitivity analysis: parameter estimates with simultaneous estimation of $\mathrm{Q}$ and $\mathrm{R}$. . . . . . . . 207

G.1 Model summary. Stock BA. . . . . . . . . . . . . . . . 209

G.2 Model summary. Stock C. . . . . . . . . . . . . . . . 210

G.3 Model summary. Stock GE. . . . . . . . . . . . . . 211

G.4 Model summary. Stock IBM. . . . . . . . . . . . . . . . 212

G.5 Model summary. Stock INTC. . . . . . . . . . . . . . . . . 213

G.6 Model summary. Stock JPM. . . . . . . . . . . . . . . 214

G.7 Model summary. Stock MMM. . . . . . . . . . . . 215

G.8 Model summary. Stock MRK. . . . . . . . . . . . 216

G.9 Model summary. Stock MSFT. . . . . . . . . . . . . . . . . 217

G.10 Model summary. Stock XOM. . . . . . . . . . . . . . . . 218

H.1 Prediction results from the time varying univariate model with estimation window size of 20 observations. . . . . . . . . . 219

H.2 Prediction results from the time varying univariate model with estimation window size of 30 observations. . . . . . . . . 220

H.3 Prediction results from the time varying univariate model with estimation window size of 50 observations. . . . . . . . 220

H.4 Prediction results from the time varying univariate model with estimation window size of 100 observations. . . . . . . . 221

H.5 Prediction results from the time varying univariate model with estimation window size of 200 observations. . . . . . . . 221

H.6 Prediction results from the "average" time varying univariate model. 222 


\section{List of Figures}

5.1 Realized and predicted returns, using Harvey's daily effects model.

Stock: BA. . . . . . . . . . . . . . . 106

5.2 Realized and predicted returns, using dummy variable daily effects model. Stock: BA. . . . . . . . . . . . . . . . . 107

D.1 Univariate model predictions and realized values of stock returns.

Stock: BA . . . . . . . . . . . . . . . . . . 164

D.2 Univariate model predictions and realized values of stock returns.

Stock: C . . . . . . . . . . . . . . . . 165

D.3 Univariate model predictions and realized values of stock returns.

Stock: GE . . . . . . . . . . . . . . . . . 166

D.4 Univariate model predictions and realized values of stock returns.

Stock: IBM . . . . . . . . . . . . . . . 167

D.5 Univariate model predictions and realized values of stock returns.

Stock: INTC . . . . . . . . . . . . . . . . . . 168

D.6 Univariate model predictions and realized values of stock returns.

Stock: JPM . . . . . . . . . . . . . . . . 169

D.7 Univariate model predictions and realized values of stock returns.

Stock: MMM . . . . . . . . . . . . . . . . 170

D.8 Univariate model predictions and realized values of stock returns.

Stock: MRK . . . . . . . . . . . . . . 171

D.9 Univariate model predictions and realized values of stock returns.

Stock: MSFT . . . . . . . . . . . . . . . . 172

D.10 Univariate model predictions and realized values of stock returns.

Stock: XOM . . . . . . . . . . . . . . . . . 173

E.1 Multivariate model predictions and realized values of stock returns. Stock: BA 
E.2 Multivariate model predictions and realized values of stock returns. Stock: C . . . . . . . . . . . . . . . . 182

E.3 Multivariate model predictions and realized values of stock returns. Stock: GE . . . . . . . . . . . . . . 183

E.4 Multivariate model predictions and realized values of stock returns. Stock: IBM . . . . . . . . . . . . . . . . . . . . 184

E.5 Multivariate model predictions and realized values of stock returns. Stock: INTC . . . . . . . . . . . . . . . . . 185

E.6 Multivariate model predictions and realized values of stock returns. Stock: JPM . . . . . . . . . . . . . . . . 186

E.7 Multivariate model predictions and realized values of stock returns. Stock: MMM . . . . . . . . . . . . . . . 187

E.8 Multivariate model predictions and realized values of stock returns. Stock: MRK . . . . . . . . . . . . . . . 188

E.9 Multivariate model predictions and realized values of stock returns. Stock: MSFT . . . . . . . . . . . . . . . . . 189

E.10 Multivariate model predictions and realized values of stock returns. Stock: XOM . . . . . . . . . . . . . . 190

E.11 GARCH and GJR-ARCH models stock returns forecasts. Stock: BA . . . . . . . . . . . . . . . . . 191

E.12 GARCH and GJR-ARCH models stock returns forecasts. Stock: C 192

E.13 GARCH and GJR-ARCH models stock returns forecasts. Stock: GE . . . . . . . . . . . . . . . . . . . 193

E.14 GARCH and GJR-ARCH models stock returns forecasts. Stock: IBM . . . . . . . . . . . . . . . . . . . . . . . . . . 194

E.15 GARCH and GJR-ARCH models stock returns forecasts. Stock: INTC . . . . . . . . . . . . . . . . . . . 195

E.16 GARCH and GJR-ARCH models stock returns forecasts. Stock: JPM . . . . . . . . . . . . . . . . . . . 196

E.17 GARCH and GJR-ARCH models stock retums forecasts. Stock: MMM . . . . . . . . . . . . . . . . . . . 197

E.18 GARCH and GJR-ARCH models stock returns forecasts. Stock: MRK . . . . . . . . . . . . . . . . . . . . . 198

E.19 GARCH and GJR-ARCH models stock returns forecasts. Stock: MSFT . . . . . . . . . . . . . . . . . . . . 199

E.20 GARCH and GJR-ARCH models stock returns forecasts. Stock: XOM ........................ 200 
H.1 Time varying parameter model forecasts for selected estimation window sizes. Stock: BA . . . . . . . . . . . . . . . 223

H.2 Time varying parameter model forecasts for selected estimation window sizes. Stock: C . . . . . . . . . . . . . . . . 224

H.3 Time varying parameter model forecasts for selected estimation window sizes. Stock: GE . . . . . . . . . . . . . . 225

H.4 Time varying parameter model forecasts for selected estimation window sizes. Stock: IBM . . . . . . . . . . . . . . . 226

H.5 Time varying parameter model forecasts for selected estimation window sizes. Stock: INTC . . . . . . . . . . . . . . . 227

H.6 Time varying parameter model forecasts for selected estimation window sizes. Stock: JPM . . . . . . . . . . . . . . 228

H.7 Time varying parameter model forecasts for selected estimation window sizes. Stock: MMM . . . . . . . . . . . . . . 229

H.8 Time varying parameter model forecasts for selected estimation window sizes. Stock: MRK . . . . . . . . . . . . . . . 230

H.9 Time varying parameter model forecasts for selected estimation window sizes. Stock: MSFT

H.10 Time varying parameter model forecasts for selected estimation window sizes. Stock: XOM . . . . . . . . . . . . . . 232

H.11 Time varying parameter univariate model: parameter evolution. Stock: BA . . . . . . . . . . . . . . . . 233

H.12 Time varying parameter univariate model: parameter evolution. Stock: C . . . . . . . . . . . . . . . . . . 234

H.13 Time varying parameter univariate model: parameter evolution. Stock: GE . . . . . . . . . . . . . . . . . 235

H.14 Time varying parameter univariate model: parameter evolution. Stock: IBM . . . . . . . . . . . . . . . . . 236

H.15 Time varying parameter univariate model: parameter evolution. Stock: INTC . . . . . . . . . . . . . . . . . . . 237

H.16 Time varying parameter univariate model: parameter evolution. Stock: JPM . . . . . . . . . . . . . . . . . . 238

H.17 Time varying parameter univariate model: parameter evolution. Stock: MMM . . . . . . . . . . . . . . . . . . . . . 239

H.18 Time varying parameter univariate model: parameter evolution. Stock: MRK . . . . . . . . . . . . . . . . . . 240

H.19 Time varying parameter univariate model: parameter evolution. Stock: MSFT . . . . . . . . . . . . . . . . . . 241 
H.20 Time varying parameter univariate model: parameter evolution. Stock: XOM . . . . . . . . . . . . . . . . . . . . . 242

H.21 Time varying $Q$ estimate vs. GARCH and GJR-ARCH variance forecasts. Stock: BA . . . . . . . . . . . . . . . 243

H.22 Time varying $Q$ estimate vs. GARCH and GJR-ARCH variance forecasts. Stock: C . . . . . . . . . . . . . . . . . 244

H.23 Time varying $Q$ estimate vs. GARCH and GJR-ARCH variance forecasts. Stock: GE . . . . . . . . . . . . . . 245

H.24 Time varying $Q$ estimate vs. GARCH and GJR-ARCH variance forecasts. Stock: IBM . . . . . . . . . . . . . . . . . . 246

H.25 Time varying $Q$ estimate vs. GARCH and GJR-ARCH variance forecasts. Stock: INTC . . . . . . . . . . . . . . . . 247

H.26 Time varying $Q$ estimate vs. GARCH and GJR-ARCH variance forecasts. Stock: JPM . . . . . . . . . . . . . . . . . 248

H.27 Time varying $Q$ estimate vs. GARCH and GJR-ARCH variance forecasts. Stock: MMM . . . . . . . . . . . . . . . . . 249

H.28 Time varying $Q$ estimate vs. GARCH and GJR-ARCH variance forecasts. Stock: MRK . . . . . . . . . . . . . . 250

H.29 Time varying $Q$ estimate vs. GARCH and GJR-ARCH variance forecasts. Stock: MSFT . . . . . . . . . . . . . . . 251

H.30 Time varying $Q$ estimate vs. GARCH and GJR-ARCH variance forecasts. Stock: XOM . . . . . . . . . . . . . 252 


\section{Chapter 1}

\section{Introduction}

Fama, in his 1991 review "Efficient Markets 2" [Fama, 1991], calls market efficiency "to be the simple statement that security prices fully reflect all available information", and defines an efficient market as one in which "prices reflect information to the point where the marginal benefits of acting on information do not exceed the marginal costs".

Evidence on market efficiency has been mixed over the years. The best evidence of market efficiency seems to be given by event studies: a large amount of research has found that prices adjust very quickly to new information. Strong form efficiency also seems to hold quite well: there is no clear indication on the ability of fund managers to outperform the market (even with the help of inside information). On the other hand, the legendary ability of the Value Line index to outperform the market remains a mystery ([Shelton, 1967, Copeland and Mayers, 1982, Stickel, 1985]).

The weak form hypothesis is unresolved as well. Theory argues that all relevant information is already reflected in prices at any point in time. Evidence suggests, however, that the volatility and variability of return series is inconsistent with the amount of new information arrival (or, more precisely, with what researchers think information is): stock prices move more than information arrival alone would suggest [Shiller, 1981, LeRoy, 1989]. Thus, even though event studies find that prices react quickly to new information, the "events" studied in event studies do not appear to occur frequently enough to explain the large variability observed in stock prices.

This evidence points to the possibility that other factors account for some of the variability in stock prices. In the case that unknown variables affect security prices and explain the large variance in them, two possibilities emerge: first, that 
these factors represent event-studies like factors, and thus prices adjust to them very quickly in which case markets could be considered relatively efficient, and second, that these factors are not events, but rather market guesses as to the value of the stock at any given point in time.

In order to make securities prices include "all relevant information", market participants, in aggregate, must first understand what the new information means for the security, and then, must incorporate it into prices. Information is defined here, as data encoded into a symbol or symbols for use in decisionmaking. Understanding the information is a complex process: to fully incorporate the information into security prices, not only must market participants understand the implications the information has for the firm now, but also in the future. This requires from the market participants the ability to foresee the consequences of information, and thus in fact, predict the market. The market participant can't, however, be satisfied with predicting the consequences of new information: he or she must combine the immediate effect of new information with the foreseen effect of new information on the future of the economic entity in question, into a present estimate of the state of the firm - the price of the security. Only when the market participant completes this task correctly and rationally will the price of the security be right.

If one or more of the steps in the process of pricing are processed incorrectly, the prices will not completely reflect new information, and the market will cease to be efficient: in this case arbitrage or quasi-arbitrage opportunities will exist and allow investors to profit from mis-pricing. The arbitrage activities of certain investors will then drive the price of the security back to the level consistent with efficiency.

The pricing process is complex and it can be expected that all market participants, including the arbitrageurs, will not be able to weed out all the inefficiency in the market at one time. If this is the case, the market will present consistent asset mis-pricing and statistical methods could be formulated to detect it. In the case when such mis-pricing is present, and a statistical method is able to detect it, profit opportunities should exist as long as "marginal benefits of acting on information exceed the marginal costs" [Fama, 1991], including transaction costs. It is possible, however, that only certain market participants that either have low trading costs, or that are able to transfer trading costs to others, would be able to profit from such irregularities. This issue is open to debate.

Pricing and arbitraging involve the ability to forecast the market, and thus require investment into methods designed to weed out market mis-pricing and inefficiency. One of the primary goals of a market participant is to process the information as quickly as possible to obtain meaning from the information. With the 
information revolution and globalization, market participants are presented with the problem of sifting through large amounts of data to find relevant information. It is possible that because of the large amount of information, certain information elements are discarded or overlooked, thus causing systematic mis-pricing. In order to avoid this accidental loss of relevant information, large information technology investments have been conducted in recent years to assist investors in pricing securities. However it is possible that the computer programs which have been written overlook important data, or that they include irrelevant data in pricing securities. Either type of mistake can cause mis-pricing, and thus make markets inefficient.

Based on these premises, this work proposes a new methodology and new models to predict the behaviour of stock returns. While the financial literature has focused almost exclusively on the predictability of stock market indices, the current work, in order to better predict the market, models the behaviour of individual stocks. The models test in turn the weak and semi-strong efficient market hypotheses.

The work is divided as follows. The next chapter presents a review of evidence on market efficiency gathered from the literature. In particular it talks about the theory of efficient markets, the distribution of returns, the ARCH literature and some findings from the behavioural finance literature. Chapter 2 presents a summary of the returns prediction literature and talks about regression approaches for predicting stock returns, as well as time series, and trading rule methods. Chapter 4 proposes a new approach to the problem of stock returns prediction. Finally, chapters 5 and 6 present the results of the empirical test of the new approach. The work is concluded in chapter 7. 


\section{Chapter 2}

\section{Evidence on Market Efficiency}

\subsection{Introduction}

This chapter reviews research examining the nature of financial markets to better understand the behaviour of returns. At first, the development of the theory of market efficiency is presented. This section discusses the thinking about the nature of financial markets from the beginning of empirical market research until today. Finally, to better understand the nature of financial markets, a review of the behaviour of stock returns from the point of view of returns distributions is presented, and a short review of ARCH literature is given.

\subsection{Theory of Market Efficiency}

The goal of the current work is to explore the efficiency of capital markets by examining the predictability of stock market prices. To achieve this goal, one has to ask what is efficiency and how should efficient markets function? In order to answer this question, this section reviews the development of theoretical work on efficient financial markets from an historical perspective.

\subsubsection{Bachelier, 1900}

In his work "Theory of Speculation", L. Bachelier [Bachelier, 1900], developed the first rigorous theory of speculative behaviour on the stock exchange and tested the model against behaviour observed on the stock market. His study distin- 
guished itself at the time by the highly rigorous mathematical treatment of proposed ideas.

Bachelier's goal was to establish a model of option prices on the French stock market. In order to achieve this goal, he first needed to develop a thorough theory of price behaviour. His insight into the functioning of financial markets has had an impact on the the theory and practice of finance that continues to this day.

Bachelier's first observation about financial markets and the stock market in particular was that exact modelling and predicting the behaviour of financial prices seemed impossible because of the quantity of influences that affect prices. He noticed that prices were affected not only by past, current and discounted future events but also by the trends and rumours in the market in such a way that no single event had any observable influence on price changes. He concluded that at any given moment, the meaning of the available information was unclear to investors, and that there were divergent opinions as to the future movement of the market, such that investors could expect a market rise or fall at any given moment in time.

Bachelier observed, however, that investors could estimate the probability of a given change in price, even though they could not predict the exact price change itself. It was thus possible to establish "a law of probability consistent with the market at any given time" ([Cootner, 1964] p 26), or to describe the "static state" of the market by determining the probabilities of price changes at any given moment in time, even though it was impossible to construct a precise model of market dynamics.

Bachelier recognized two types of probabilities of stock price changes: a mathematical (or a priori) probability, and a subjective probability which every speculator established given his beliefs and information. The subjective probability is the investor's expectation of market movement given all information available to him or her. This expectation involves the analysis of current news and announcements and the discounting of future events. The result of this process is such that buyers and sellers have opposing views of future market movement, and "at a given instant, the market believes in neither a rise nor a fall of true prices". The mathematical probability is an a priori measure of price movement. Since the market can move in any direction at any moment, Bachelier concludes that, a-priori, "the mathematical expectation of a speculator is zero".

Based on the assumption of zero expectation, Bachelier then derived the theoretical behaviour of prices in the stock market. He argued that the probability of a price occurring is a function of the price itself, and that the price with the greatest probability of occurrence is the current price. He bypassed the issue of limited 
liability and assumed that prices can vary in $(-\infty, \infty)$, by arguing that the probability that the price would drop to less than zero was negligible, and assumed that the probability distribution function was symmetric around the current price.

He defined the probability that the price is in the interval $(x, x+\mathrm{d} x)$ at time $t$ as $p_{x, t} \mathrm{~d} x$. He then found the conditional probability, that, at time $t_{1}+t_{2}$, the price will be equal to $z$ given that it was $x$ at time $t_{1}$ to be $p_{x, t_{1}} p_{z-x, t_{2}} \mathrm{~d} x \mathrm{~d} z$. Because the price at time $t_{1}$ can be anywhere in the range $(-\infty, \infty)$, the probability that the price will be $z$ at time $t_{1}+t_{2}$ is:

$$
p_{z, t_{1}+t_{2}} \mathrm{~d} z=\int_{-\infty}^{+\infty} p_{x, t_{1}} p_{z-x, t_{2}} \mathrm{~d} x \mathrm{~d} z, \quad \text { or, } p_{z, t_{1}+t_{2}}=\int_{-\infty}^{+\infty} p_{x, t_{1}} p_{z-x, t_{2}} \mathrm{~d} x
$$

The function that satisfies this equation is: $p=A \exp \left[-B^{2} x^{2}\right]$. Bachelier noted that $\int_{-\infty}^{+\infty} p \mathrm{~d} x=\int_{-\infty}^{+\infty} A \exp \left[-B^{2} x^{2}\right]=A \int_{-\infty}^{+\infty} \exp \left[-B^{2} x^{2}\right]=1$ since the area under a probability distribution function is unity. This integral yields a value of $\sqrt{\pi} / B$, and thus, $A \sqrt{\pi} / B=1$ which gives $B=A \sqrt{\pi}$, which substituting in the original function yields $p=A \exp \left[-\pi A^{2} x^{2}\right]$. If one sets $x=0$, one obtains the probability of the current price $p_{0}=A$, and substituting it into the original formula gives the desired probability function $p=p_{0} \exp \left[-\pi p_{o}^{2} x^{2}\right]$. This is a form of the Normal probability distribution, where $p_{0}$ depends on time. After further manipulation, one can prove that $p_{0}=H / \sqrt{t}$, where $H$ is a constant. Substituting this result yields $p=H / \sqrt{t} \exp \left[-\pi H^{2} x^{2} / t\right]$, and computing the expectation of this function yields $\int_{-\infty}^{+\infty} x \cdot H / \sqrt{t} \exp \left[-\pi H^{2} x^{2} / t\right] \mathrm{d} x=\sqrt{t} /(2 \pi H)$. Evaluating this expectation at $t=1$ and substituting, yields $k=1 /(2 \pi H)$, or, $H=1 /(2 \pi k)$, and gives the final form of the probability:

$$
p=\frac{1}{2 \pi k \sqrt{t}} \exp \left(-\frac{x^{2}}{4 \pi k^{2} t}\right)
$$

Finally, the expectation, $\int_{-\infty}^{+\infty} p x \mathrm{~d} x=k \sqrt{t}$, implies that the expected price $z$ at time $t_{1}+t_{2}$ is a function of the time interval and not of the starting price. This important result meant that the future prices did not depend on past prices, but only on time, and implied that one could not predict the behaviour of future prices from looking at past prices.

\subsubsection{Taussig, 1921}

Taussig [Taussig, 1921] considered buyers and sellers in an organized market to be members of the same group all behaving in the same manner by arguing that 
a "potential buyer who is likely to be brought into the market by a lower price is also a potential seller likely to be brought in by a higher price". He disputed the basic assumption in economics that lower price incites higher demand, and argued that, as price falls, less can be demanded not more. He illustrated this point by the actions of speculators on commodity markets who hope for a price decline by selling commodities short, and thus inciting a "bearish" run.

He advocated the concept of "penumbra", a range of prices in which the workings of demand and supply are not known, where prices can change independently of the supply and demand of goods, and may be moved by fads, trends and rumours. The idea that Taussig proposed is based on the observation that prediction of the supply and demand of goods is very difficult, and thus so is the establishment of a price. Taussig illustrated his point with examples of markets for goods such as eggs, potatoes, wheat, and cotton. He argued that in such a market, the demand and supply cannot be known exactly, but only estimated, and thus prices cannot be determined with precision. The higher the uncertainty about the amount of supply and demand, the greater will be the uncertainty about the price, and the wider the penumbra for possible price variation. Speculators and suppliers on the market will gamble on the uncertainties and influence price movement, possibly, irrationally, that is, without regard to fundamental factors affecting the value of the commodity. While the speculator's actions may move the price, the price will not be able to leave the penumbra. Taussig argued that even though there was uncertainty about the supply and demand, enough information was available for all the market participants to agree about a general price level. If the price of goods exited outside of the penumbra, the speculators' actions would push it back into it.

From this point of view, the equilibrium price is no longer a point estimate, but can belong anywhere inside a price interval, the penumbra. Within the penumbra, speculative pressures can move the price up and down, but within certain limits: the lower and upper edges of the price interval - once either interval is exceeded, market forces will push the price back into the penumbra.

Taussig further argued that in the stock market, there are no clear limits on the fluctuation of prices, unlike for commodities (such as wheat, cotton or steel) which serve directly human needs. The interval of price uncertainty is thus much wider in the stock market. Stock speculation is also different from speculation on commodities in the sense that, instead of acting on tangible objects, it facilitates the development of new technologies and improvement of old ones by dividing risk and limiting liabilities. The limits to price fluctuation are established by the future eamings power, but since these are sometimes vague, the penumbra for 
stocks can be very large.

In summary, Taussig argues that "market equilibrium seems to be as far from stability as from predictability". He says that prices on the stock and commodities markets are not known with precision, but rather are estimated to exist in a certain price range, which can expand or contract depending on the amount of information flowing to the market. Within this interval, speculative pressures independent of the value of the asset drive its price.

\subsubsection{Working, 1958}

Working (1958) [Working, 1958] proposed an alternative model of price formation, a model based on expectations. In his model market participants anticipate future events by gathering and analyzing "available and useful information" (Taussig, p. 193).

Working's assumptions result in a realistic and treatable model. First, he assumed that although it is plentiful, information is never perfect nor exact. He also assumed that traders cannot grasp all the information available to them, and that they engage in an "informal division of labour" - each trader analysing only particular types of information. Additionally, no trader on his own can affect the prices on the market. In order to be even more realistic, Working postulated the existence of two types of participants: competent and "inept" ones. Inept traders don't analyse the data thoroughly and often "go with the market".

The nature of the anticipatory model requires traders to search for information to make better decisions, and to gain understanding of the effects of the information on future events. The traders that make good use of this information will profit. Prices in this model behave randomly because the nature of new information is unpredictable, but the price changes will be gradual, not instantaneous, thus inducing predictability in prices. Working argued for the graduality of price changes on the basis of the precautions that regulators take in announcing official crop estimates ([Working, 1958] p. 195), the goal of these precautions being to allow all traders to learn the news and to assess the impact of the new information on stock prices so that the prices can reflect it immediately. Since normal news announcements lack such characteristics, Working proposed that prices will respond gradually to new information.

In Working's model the competent traders profit by gathering information and incorporating it in prices. If a type of "useful information" is not being priced, the neglect will be discovered and a market participant will profit by incorporating it in prices. The model also allows for uninformed participants by assuming 
that prices do not respond immediately to information, in which case uninformed traders can exist by simply investing with the market even though their profits will be small.

\subsubsection{Modern Developments}

So what exactly constitutes an efficient market? Ball [Ball, 1988] attributes the first use of the term "efficient market" to the paper by Fama, Fisher, Jensen and Roll [Fama et al., 1969] - "The Adjustment of Stock Prices to New Information", in which an efficient market was defined as one where "prices adjust rapidly to new information". The problem is, however, that a speed of adjustment consistent with the concept of an efficient market has not been defined, and as the review by Fama [Fama, 1991] shows, certain studies find surprisingly slow price adjustment speeds in the order of days or even months.

In 1970, Fama, in his "Review of Theory and Empirical Work" [Fama, 1970], the first review of the state of the market efficiency hypothesis, gave a more precise definition of an efficient market as one in which "prices always 'fully reflect' available information". This definition is not representative, however, of real capital markets: it applies to a perfect capital market - one which is allocationally and operationally efficient. In such a market four conditions are met: 1) absence of transaction costs, taxes and regulation; 2) no one individual has influence on the price of securities sold; 3 ) all market participants have perfect access to free information and 4) all individuals take actions as to maximize their expected utility (Copeland and Weston [Copeland and Weston, 1992]).

In the same article Fama formalized the concept of market efficiency by identifying three types of efficiency - weak, semi-strong and strong defined with respect to three sets of information: weak form efficiency stipulates that prices cannot be predicted using their historical values alone, semi-strong efficiency further adds that prices remain unpredictable even after using other publicly available information and finally strong-form efficiency stipulates that investors cannot gain abnormal profits using inside information, apart from some investors who have the opportunity to trade at greatly reduced costs.

This operationalized categorization of market efficiency is the result of a review of early empirical studies concerned with identifying the behaviour of stock prices. Fama noticed that early literature could be classified into three compact classes: weak form tests which were mainly concerned with whether prices followed a random walk, semi-strong form tests which were concerned with the speed of adjustment to announcements, and finally strong-form tests concerned 
with trading based on private information. Fama further noted that these classes were not definitive, but were only a convenient device to find "the level of information at which the hypothesis [of market efficiency] breaks down". The classification into these exact categories is thus not necessary, and can be changed (but is rather useful).

Later, Jensen [Jensen, 1978] provided a more precise definition of market effciency: "a market is efficient with respect to an information set $\theta_{t}$ if it is impossible to make economic profits (risk adjusted returns, net of all costs) by trading on the basis of the information set $\theta_{t}$ ".

In his 1991 article, Efficient Markets 2, Fama [Fama, 1991] redefined the categories he proposed in 1970 . The first category, tests for return predictability, enlarged the scope covered by weak-form efficiency. While weak-form tests were previously concerned with the prediction of future returns based solely on past returns, the new category covers the use of fundamental and economic data on top of past returns in predicting future returns, and includes cross-sectional equilibrium pricing models, seasonality research, financial market volatility research and trading rules studies. Fama did not, however change the second and third categories of tests, but has renamed them with more descriptive titles: event studies research has replaced semi-strong form hypothesis tests, and strong form tests are now called tests for private information.

The concept of market efficiency is inherently untestable. In order to be tested, it first needs to be expressed in a model which then will be subjected to a test [Fama, 1991]. Because efficiency is not a quantitative concept, and thus cannot be expressed easily in a model, no model of market efficiency is truly perfect. The best model of efficiency is the one which captures the most of the essence of efficiency and translates it into mathematical terms. However it is not possible to create a perfect model, and thus even the best model will not refiect all the ideas of efficiency. This results in the joint hypothesis problem [Fama, 1991]. This problem appears when testing a model of retums against observed market behaviour. When the model cannot explain all encountered behaviour the source of the unexplained behaviour - market inefficiencies or a bad model - is never clear. Since a model can never express the concept of efficiency perfectly, some of the bad fit can be attributed to it, and some to market inefficiencies - it is not clear, however, in which proportion. The joint hypothesis problem stands as the most difficult issue encountered in tests of market efficiency.

All theories assume that investors are rational profit maximizers who use information to exploit profit opportunities in the stock market. The disagreements relate to the manner in which markets incorporate the information, or on how ef- 
ficient the markets are and how they achieve efficiency. According to Fama's definition, a market is efficient if it reflects all relevant information in prices. Clearly this definition is too vague.

Fama's classification of market efficiency into three classes is an attempt to facilitate the analysis of market behaviour. With this attempt, Fama formalized the testing of market behaviour by allowing researchers to look for specific deviations from theory, and for confirming theories. It is thanks to this formalization that progress in the quest for understanding and explaining the behaviour of investors and the stock market has occurred in the last thirty years.

\subsection{Distributional Properties of Returns}

When first presented with a sample of data, a researcher's first instinct is to graph it, look at it, compute its mean, mode, median, standard deviation and statistics which measure skewness and kurtosis, or in other words, attempt to describe its distribution. In some cases, the researcher has a preconceived idea of the behaviour of a random variable, and through statistical testing needs to determine whether the data agrees with this hypothesis. Such a process has been followed in the analysis of numerous natural phenomena, including that of stock prices and returns.

Research on the distribution of stock returns started with Bachelier's [Bachelier, 1900] work "The Theory of Speculation". He realized that an exact deterministic description of the behaviour of stocks could not be performed, but that instead, a probabilistic description could be achieved, and proposed that one could establish "a law of probability consistent with the market at any given time" ([Cootner, 1964] p. 26). Bachelier then proceeded with a careful analysis of speculator behaviour to show that stock prices will follow the normal probability distribution ([Cootner, 1964] p. 30).

The proof that returns, in a financial market populated by rational speculators, are distributed normally, has entrenched the use of the normal probability distribution function in financial research. Two additional reasons have contributed to the popularity of the normal: first, normality facilitates mathematical modeling and second, numerous statistical methods require the assumption of normality [Kon, 1984].

Early on, the literature presented a rich portrait of the behaviour of stock returns. In one of the first economic studies using the computer Kendall

[Kendall, 1953] analyzed time series of stock indices and commodity prices. Con- 
structing bivariate distributions of first differences of prices of wheat, cotton, and stock indices for consecutive time periods, he found the series to be "approximately" normally distributed. The stock prices told a varied story - it was found that the bivariate distribution of some indices corresponded to the normal. Some other indices, however, in particular the index of "Investment Trusts", showed a significant amount of dependence. Wheat prices, on the other hand, presented a perfect image of normality. A subsample analysis of weekly wheat prices for two separate periods, 1883-1914 and 1921-1934, indicated that, for both periods, the bivariate distribution of first differences was close to normal. A closer analysis suggested a different picture - subsample analysis of the higher moments of the empirical distribution uncovered a pattern of change, in particular for the variance (see Table 2.1): an increase in variance of wheat prices occurred after World War 1. Kendall concluded: "We have here an interesting and rather unusual case of a time series for which the mean remains constant but the variance appears to be increasing". Monthly wheat prices, however, seemed to approximate normality better. The ratio of monthly to weekly variances yielded a value of $2.82 \mathrm{vs}$. a theoretical value of 2.75 , suggesting that the series follows a random walk. Similar conclusions were reached by Working [Working, 1934]. After attempting to reproduce a wheat price series by summing normally distributed random variables, Working concluded that they would be better approximated by a process generated from a skewed distribution with changing variance rather than the normal law.

\begin{tabular}{lcccc}
\hline Moment & $\begin{array}{c}1883-1914 \\
\text { weekly }\end{array}$ & $\begin{array}{c}1921-1943 \\
\text { weekly }\end{array}$ & $\begin{array}{c}1883-1934 \\
\text { weekly }\end{array}$ & $\begin{array}{c}1883-1934 \\
\text { monthly }\end{array}$ \\
\hline$\eta_{1}$ & 0 & 0.0964 & 0.0289 & 0.0504 \\
$\eta_{2}$ & 7.75 & 22.77 & 12.32 & 34.83 \\
$\eta_{3}$ & 12.67 & 34.58 & 20.27 & 6.42 \\
$\eta_{4}$ & 657.3 & 2754.9 & 1293 & 6914 \\
Skewness & 0.34 & 0.101 & 0.219 & 0.00151 \\
Kurtosis & 10.856 & 5.3 & 8.5 & 5.07 \\
\hline \hline
\end{tabular}

Table 2.1: Central moments of wheat prices (Kendall [Kendall, 1953])

In an innovative study of the behaviour of stock market prices, Osborne [Osborne, 1959] derived the behaviour of prices in an ideal market, and was one of the first to compare it to observed behaviour. His analysis first focused on the nature of prices. Plots of one-day closing prices for 1000 NYSE stocks revealed 
that prices were skewed to the right and censored at 0 , suggesting a log-normal price distribution. A histogram of log-prices revealed the normal curve, and an analysis of monthly and yearly log-price differences showed them to be quasinormally distributed. Like Bachelier, he analyzed the hypothetical behaviour of rational investors, and found that the stream of returns generated in a rational market will be distributed normally with mean zero, and variance proportional to the square root of the investment horizon.

Faced with this evidence, Mandelbrot [Mandelbrot, 1963] identified four problems with the Gaussian description of stock returns: 1) normality cannot explain the large frequency of big price changes, or thick tails, nor the high peaks of the empirical distribution of returns, 2) sudden price changes are common, 3) prices appear to be predictable, and 4) stationarity does not provide an appropriate description of price behaviour. Unsatisfied with the Gaussian model's fit to the distribution of financial returns, he proposed a generalization of Bachelier's model to explain the excess kurtosis observed in the data.

Mandelbrot's contribution was to propose the stable Paretian family for the distribution of stock returns while, at the same time, maintaining the independence of returns hypothesis. The logarithm of the characteristic function of the stable Paretian distribution is:

$$
\log \int_{-\infty}^{\infty} \exp ^{i t x} f(x) d x=i \delta t-\gamma|t|^{\alpha}\left[1+\frac{i \beta t}{|t|} \tan \left(\frac{\alpha \pi}{2}\right)\right]
$$

The function has four parameters: $\alpha \in[0,2]$, the characteristic exponent, controls the size of the tails of the distribution, $\beta \in[-1,1]$ determines the skewness of the distribution, $\gamma$ is the scale parameter, and $\delta$ represents the expectation. The distribution is symmetrical about the location parameter $\delta$ when $\beta=0$. The distribution's first moment exists when the characteristic exponent $\alpha>1$ and the second moment exists when $2 \leq \alpha<3$. The stable Paretian distribution with parameters $\alpha=2, \delta=\mu, \gamma=\sigma^{2} / 2$, corresponds to the normal distribution.

In particular, Mandelbrot's model has the following properties. First, the normal law is a particular case of the stable distribution when the characteristic exponent $\alpha=2$. Secondly, for $\alpha<2$, the area under the tails increases, but at the same time the variance of the distribution becomes infinite. Two immediate consequences of the new model are that the markets will be much more volatile, and that new statistical tools will be needed to analyze the behaviour of stocks. Methods assuming normality are seen as obsolete. This implies that the large body of knowledge accumulated with the help of the assumption of the normal distribution function may be invalid. In particular, the application of the least squares 
method will yield non-robust results, and forecasting will be made difficult by the unavailability of a prediction error measure based on the existence of a second moment. However, Mandelbrot suggested that the absolute value error may be a solution to this problem ([Mandelbrot, 1963] p. 323). Spectral methods also become unusable in this new framework.

The behaviour of a market under the stable Paretian hypothesis is very different from that stipulated by the Gaussian model. The new model stipulates that variance can be infinite, which implies that the market will be much more volatile and risky: in a stable Paretian market, price changes will not be gradual but rather sudden. The increased volatility will affect the behaviour of stock prices in several ways. One implication directly affects the existence of trends. Trends can be defined as prolonged periods during which the price slowly rises or falls: in a stable Paretian market, trends will not be caused by small changes in prices, but rather by a few large movements, therefore becoming an illusion. Another implication of the hypothesis is that trading rules which rely on smooth price changes, such as those of Alexander [Alexander, 1961], will not be profitable.

Mandelbrot [Mandelbrot, 1963, Mandelbrot, 1967] examined the behaviour of cotton, wheat, and railroad stock prices, and interest and exchange rates. Using daily cotton prices for 1900-1905 and 1944-1958 and monthly data for 18801940 , assuming the distribution of returns to be symmetrical with $\beta=0$, mean 0 , and, $\delta=0$, and, using a graphical estimation procedure, he found that the prices were best described by a stable Paretian distribution with characteristic parameter $\alpha=1.7$. Examining daily data in more detail, Mandelbrot also found that the $\gamma$ parameter decreased between 1900 and 1950 indicating that prices became less "volatile" during the period. When examining the series of monthly returns over the period 1816-1940, Mandelbrot also found that the Paretian law describes the returns well, however, he noticed that after the civil war the distribution of returns changed suggesting mixtures of Paretian distributions would provide a better fit to the data. Examination of the wheat, railroad, and rates series yielded similar results.

Blattberg and Gonedes [Blattberg and Gonedes, 1974] attempted to describe the fat tails of the distribution of returns using two distribution functions having this ability: the stable Paretian and Student distributions. Their goal was to identify the model providing the best fit.

Model selection from among the two contender distributions has important implications for the behaviour of returns. If the Student distribution with degrees of freedom parameter $\nu>2$ fits the distribution of returns adequately, then the central limit theorem dictates that the cumulative return will tend to be distributed 
normally as the compounding time grows. This is not the case if data are distributed according to the stable Paretian distribution with characteristic exponent $\alpha<2$ : in this case the cumulative return will also be distributed according to the stable Paretian law. For example, if daily data are t-distributed, monthly data will likely follow the normal distribution, while this will not be the case under the stable Paretian law. The selection of a distribution function thus has implications for analysis tools. In the case data are t-distributed, standard methods can be used to model longer term returns, but this will not be the case under the stable Paretian model.

Blattberg and Gonedes estimated the Student model with the help of maximum likelihood to find the parameters $m, H$ and $\nu$, the degree of freedom parameter. The form of the likelihood used was:

$$
L\left(m, H, \nu ; x_{1}, \ldots, x_{n}\right)=\prod_{i=1}^{n} \frac{\nu^{\frac{1}{2} \nu}}{\beta\left(\frac{1}{2}, \frac{1}{2} \nu\right)} \cdot\left[\nu+H\left(x_{i}-m\right)^{2}\right]^{-\frac{1}{2}(\nu+1)} \sqrt{H}
$$

To estimate the stable Paretian model, they used the Fama and Roll estimator [Fama and Roll, 1968, Fama and Roll, 1971] to avoid the computational difficulties associated with the maximum likelihood estimator for the stable Paretian laws.

The data for the study consisted of daily returns for the 30 Dow Jones stocks for the period 1957-1962. The estimated degrees of freedom parameter, $\nu$, for the Student model ranged from 2.53 to 13.26 over the 30 stocks, with a mean of 4.79 , while the stable Paretian model characteristic exponent, $\alpha$, ranged over the interval 1.45 to 1.87 , with mean 1.65 . When the returns are summed over a period of 5 days, the average value of $\nu$ increased to 11.22 , and the average value of $\alpha$ to 1.72. The log-likelihood ratio statistic sided with the Student model, ratios for all stocks being greater than 0 .

Using the fact that the $\nu$ parameter increased significantly when one increased the return compounding period, that the $\alpha$ parameter behaved in the same manner, and that the likelihood ratio always gave preference to the Student model, Blattberg and Gonedes concluded that the data is best described by the Student model. The strength of this conclusion is questionable, however, since the authors used a likelihood statistic to evaluate a non-maximum-likelihood estimate (the Fama and Roll estimator), a criteria which may have biased the test against the Paretian model.

One of the other popular models for stock returns is the mixture of normal distributions hypothesis. This hypothesis proposes that stocks returns are distributed normally but with parameters shifting between a finite set of alternatives. In this 
model there exist two types of parameter shifts: 1) shifts associated with structural changes, and, 2) cyclical shifts [Kon, 1984]. Structural shifts are associated with major events affecting the stock, such as mergers, capital structure changes etc. Cyclical changes on the other hand occur when the stock shifts systematically between sets of parameters, such as in the case of the day of the week effect and other seasonal announcements.

Hsu, Miller and Wichem [Hsu et al., 1974] argued that stable Paretian distributions do not provide a proper description of stock market returns, and that their good fit is simply an illusion. They proposed that instead, stock market returns could be adequately characterized by a normal distribution with varying parameters, and in particular a changing variance.

In order to prove their hypothesis, Hsu, Miller and Wichern conducted tests of fit, non-stationarity and stability of the stable Paretian distribution on daily returns data from 4 major companies (AT\&T, Boeing, GM and RCA) for the period January 1963 to December 1970.

The authors used the minimum $\chi^{2}$ procedure to estimate the parameters $\alpha$ and $\gamma$ for the stable Paretian distribution for each of the four stocks. To facilitate the computations, they used the Fama-Roll estimate for the parameter $\delta$, and assumed that the distribution is symmetric with parameter $\beta=0$. The parameter values were chosen so as to minimize the $X^{2}$ criterion: $X^{2}=\sum_{i=1}^{i=13}\left(n_{i}-n_{i}^{*}\right)^{2} / n_{i}^{*}$, where $n_{i}$ and $n_{i}^{*}$ are the observed and expected frequencies of returns in class $i$.

The comparison of $X^{2}$ with the theoretical distribution did not yield favorable results. While the estimated values of $\alpha$ are less than 2 (on average 1.7) for the four companies, the $\chi^{2}$ significance test indicated that "symmetric stable Paretian distributions are not consistent with the data". The cause of the bad fit was the inability of the stable Paretian distribution to fit the central area of the distribution of returns and the over-fitting of the tails.

Hsu, Miller and Wichern next examined the stable Paretian distribution with respect to 420 monthly returns for 20 major corporations, again using the $\chi^{2}$ procedure. The results for the monthly data, unlike for daily data, were consistent with the stable Paretian distribution.

To test the stable Paretian fit of the monthly data in more depth, the authors test the stability of the fit over $1926--1941$ and $1942--1960$. The results were intriguing. The estimates of the parameters $\gamma$ and $\alpha$ were found to be very different in the two periods: for the pre-war period, the characteristic exponent never exceeded 1.6 for any of the twenty companies considered, however for the post-war period, the characteristic exponent increased for all the companies by 0.27 . Further tests suggested that the stable Paretian distributions provided a good 
fit for the pre-war period, but that the normal distribution was more adequate for post-war data.

The authors concluded that the fit of the stable Paretian functions is "misleading", and does not describe appropriately the nature of the data. They suggested that instead, researchers should look at the data as non-stationary in nature, and model the data accordingly.

Boness, Chen and Jatusipitak [Boness et al., 1974] argued that stock returns may be affected by changes in the capital structure of the firm. They believed that stock returns follow a random walk, but that capital structure changes affect the parameters of the random walk which results in a "contaminated distribution" for stock returns. A contaminated distribution is a distribution similar to the normal distribution but with thicker tails, due to the data being drawn from distinct populations. The author's hypothesis was that the distribution of returns believed to be stable Paretian due to the long tails, is in fact normal with non-stationary parameters, in particular the return's variance.

The authors used an event-study type analysis to examine the evolution of the distribution of 33 electric utilities which have had capital structure changes by issuing debt in the period 1961-1968. The data consisting of 130 Friday closing prices, spanning 2 years before and 2 years after the capital structure change for each firm, was analyzed in three steps: the whole period, and the before and after capital structure change periods.

Several $\chi^{2}$ tests of normality, skewness and kurtosis tests were carried out on the three data periods for all stocks. The three tests showed different results for the combined time period and for the two sub-periods. For the whole time period, tests tended to reject the hypothesis of normality and standard skewness and kurtosis, while for each of the sub-periods, the tests tended to find less evidence of deviations from normality. For example, the $\chi^{2}$ test rejected normality for 15 stocks for the whole period, while for the pre-change period it rejected normality for only 8 stocks, and 11 stocks for the post-change period, with a similar story being told by skewness and kurtosis tests. In order to examine this phenomenon more closely, Boness, Chen and Jatusipitak computed the $\alpha$ coefficient of the stable Paretian distribution for every stock, for the whole, and before and after change periods. The results for the whole period showed $\alpha<2$ for every stock, and indicated that data was stable Paretian. Sub-period results found however the $\alpha$ coefficient equal to 2 for 11 stocks for the pre-change and 9 for the post-change periods, indicating agreement with normality. Further tests indicated that normality may be the real underlying generating process for the distribution of returns: tests of stability of the mean and variance for the pre and post change periods 
found that for a majority of stocks, the two parameters were significantly different. This result mirrored those of Hsu, Miller and Wichern who found that while the stable Paretian distribution described well their whole data sample, it failed to do so for the post-war sample. These results seem to show that, while the stable Paretian distribution may fit a data set, it may not explain it if the parameters of the underlying distribution change in time.

To further examine stock behaviour, the authors fitted the market model to the three periods of data. Using the Chow test for stability of the regression coefficients between the pre and post periods, the authors found that the coefficients are significantly different for 29 of the 33 stocks. Next, they examined the regression residuals for changes in mean and variance. The cumulative average abnormal returns (CAR) over all stocks were found to have a different behaviour before and after the capital structure change: before the change, the CAR did not show any abnormal trends, however post-change the CAR showed a definite upward trend caused by positive regression residuals. Sorting stocks by the size of the capital structure change, the authors found that the cumulative returns were the highest for the group for which leverage increased the most, and were negative for the small change group. Examining squared residuals, the authors found a similar pattern: post-change the variability of the residuals displayed an increasing trend, while pre-change there was almost no variability in residuals.

These results seem to confirm those of Hsu, Miller and Wichern who concluded that returns may be non-stationary. The results of Boness, Chen and Jatusipitak showed that as the capital structure changed so did the distribution of the returns for stocks. This indicates that changes in other aspects of the firm may also provoke changes in the return generating process and the parameters of the returns distribution.

Kon's [Kon, 1984] study compared Gaussian mixture and Student models of stock returns. Kon [Kon, 1984], similarly to Boness, Chen and Jatusipitak

[Boness et al., 1974], proposed that the true distribution of stock returns could be normal with it's parameters shifting between a finite set of alternatives. He identified two types of events causing shifts in parameters: time-ordered shifts associated with capital structure changes, acquisitions and exogenous events, and cyclical shifts between sets of parameters - such as day of week and seasonal announcements.

The analysis was performed on 18.5 years of daily data on the 30 DJIA stocks, and the SP500, CRSP Equally Weighted and Value Weighted indices. In order to capture structural and cyclical effects in the data, the author sorted it by year - for time ordered events, by day of week - for cyclical events, and by year and day of 
week.

Preliminary analysis showed that the original data presented signs of high skewness and kurtosis. The author hypothesized that skewness in returns was caused by shifts in the mean of returns, while kurtosis by the shift in the variance. Results using data sorted by year, day of week, and year and day of week were close to the normal distribution. Additionally, small firms were found to have the most deviations from normality.

The authors next fitted the data with mixtures of normal distributions, starting with mixtures of two normal distributions, and augmenting the model successively to 3, 4 and 5 normal distributions. The models showed that out of the 30 DIIA stocks, 7 could be described with a mixture of 4 normal distributions, 11 with a mixture of 3 , and 12 with a mixture of 2 normal distributions. The results also revealed an intriguing fact: each stock model contained one normal distribution with a negative mean parameter: the author explained the negative mean as being consistent with the Monday effect. Stability tests rejected the hypothesis of a stationary mean and variance for 27 of 30 DJIA stocks and 2 out of the 3 indices considered with the constant variance hypothesis being rejected the most often.

Kon next fitted the Student model to the data, and compared its fit to the mixture model. He found that for 23 stocks and the 3 indices, the mixtures model explain the behaviour of returns better as long the the number of normal distributions in the mixture were equal to or exceeded 2. This result was to be expected since a single normal distribution could not explain the data as well as the Student distribution, because of the thick tails. Overall the study concluded that the normal mixture model is a better descriptor of stock returns than the Student model because returns are not stationary in time.

\subsection{Volatility Research}

One of the most interesting and intriguing behaviors exhibited by stock prices is the variability of stock returns. In the early literature, Kendall had already remarked that the variance of the first differences of wheat prices has increased in the post-First World War period as compared to the pre-war years. Kendall concluded that the series in question was peculiar in the sense that "the mean remains constant but the variance appears to be increasing" [Kendall, 1953].

Curiously, the theory of financial markets, starting with Bachelier, is silent on the subject. One of the oldest theories for the presence of volatility is the work by Taussig [Taussig, 1921] in which the author suggests that prices are not 
determined exactly, but rather exist in a "penumbra" the size of which can vary over time together with uncertainty about the underlying value of the asset being priced.

In an influential article, Black [Black, 1976] attempted to measure the relationship between stock retums and return volatility as measured by the variance of stock returns over a period of time. Using daily data, Black first regressed the stock returns on the future one period change in the variance of returns, and found a negative relationship between the two variables - positive changes in prices seem to be associated with significantly reduced volatility: "a $1 \%$ summed return seems to imply a more than $1 \%$ volatility change". Additionally, stocks with low volatility seem to be affected by changes in prices more than high volatility stocks. The most interesting results showed high volatility to persist over a period of time, and then diminish gradually.

ARCH models represent a methodological development in the quest to explain and model the heteroscedastic behaviour of some economic phenomena, and in particular that of financial returns. The ARCH models introduced by Engle [Engle, 1982] allow for modeling of time series with time-varying variances, such as stock returns. While conventional time series models assume the variance of the time series to be constant in time, autoregressive conditional heteroscedastic models allow the variance of the time series to be influenced by it's own past. Letting $y_{t}$ represent a random variable, and $\psi_{t}$ a set of explanatory variables, Bollerslev's [Bollerslev, 1986] GARCH(p,q) model can be written as:

$$
\begin{aligned}
y_{t} \mid \psi_{t} & \sim N\left(x_{t} \beta, h_{t}\right) \\
\epsilon_{t} & =y_{t}-x_{t} \beta \\
h_{t} & =\alpha_{0}+\sum_{i=1}^{p} \alpha_{i} \epsilon_{t-i}^{2}+\sum_{i=1}^{q} \beta_{i} h_{t-i}
\end{aligned}
$$

where, $h_{t}$ represents the time varying variance. The GARCH(p,q) model reduces to the $\mathrm{ARCH}(\mathrm{p})$ model for $q=0$, and for $p=q=0$, it reduces to a white noise process with variance $h_{t}=\alpha_{0}$.

Nelson [Nelson, 1991] extended the ARCH framework in order to better describe the behaviour of return volatilities. Essentially, three main issues were identified. The first issue with the ARCH model concerns its ability to correctly describe the relation between stock returns and stock returns variance. The ARCH models describe the conditional variance as a function of lagged $\epsilon_{t}^{2}$ : this means that the conditional variance is only a function of the magnitude of returns. Unfortunately, the variance behaviour is more complex, and it responds in a different 
manner to positive and negative returns - this type of behaviour is missed by the ARCH models for whom only the magnitude, and not the sign of returns matters. The positivity constraints on ARCH model parameters represent another issue since they effectively restrict the behaviour of the variance. The solution proposed by Nelson to remedy these problems is a reformulation of the conditional variance equation into:

$$
\ln \left(h_{t}^{2}\right)=\alpha_{0}+\sum_{i=1}^{\infty} \beta_{i}\left[\theta \epsilon_{t-i}+\gamma\left(\left|\epsilon_{t-i}\right|-\mathbb{E}\left|\epsilon_{t-i}\right|\right)\right] .
$$

This representation has the advantage of forcing the conditional variance to remain positive, while allowing it to be a function of the size as well as the sign of retums, and thus permitting the variance to respond asymmetrically to positive and negative shocks. Moreover, this representation does not require bounds on the parameter space since the positivity requirement for volatility is always maintained.

Nelson proceeded to fit the model to daily value-weighted CRSP index data for the period July 1962 to December 1987. The model proposed an ARMA structure to the conditional variance, and used the following model for the excess returns:

$$
R_{t}=a+b R_{t-1}+c h_{t}^{2}+\xi_{t}
$$

Assuming a Generalized Error distribution (GED) for the error term, Nelson used the Schwartz criterion to select the appropriate order for the ARMA conditional variance process: the $\operatorname{ARMA}(2,1)$ model was selected. The model yielded the following results. First, the model found a weakly significant negative relation between returns and return variance with $c=-3.4$. Secondly, there was found to be an important difference between the effects of positive and negative excess returns on variance: negative excess returns tended to increase variance more than positive excess returns. The study of estimated GED parameters also found the conditional distribution of the model's error term to be thick tailed. Nelson also found that non-trading days' variance was approximately five times lower than on a normal trading day.

Nelson's study is important due to the fact that it expanded the ARCH methodology in a new direction, breaking the rigidness of the G/ARCH specification. The most important contribution was to propose a model to test the hypothesis that the variance of returns was influenced differently by positive and negative excess returns. His study found that not only was that statement true, but also that excess returns were negatively related to stock market variance. 
Engle and $\mathrm{Ng}$ [Engle and $\mathrm{Ng}, 1993$ ] further explored the subject of asymmetric impact of news on the volatility of stock returns. The literature (see [Black, 1976, French et al., 1987, Nelson, 1991]) has reported widely on the fact that good and bad news hold different consequences for the behaviour of return volatilities: not only does the magnitude of news affect the volatility of returns, but markets seem to respond differently to good and bad news. Nelson's EGARCH sought to model this effect by allowing positive and negative excess returns to affect volatility differently. The work of Engle and $\mathrm{Ng}$ further contributed to the literature by examining the ability of the various volatility models to capture the asymmetric relation between news and return variance.

The principal diagnostic tool used in the study is a "news impact curve". It is a function relating conditional volatility $h_{t}$ to unexpected returns $\epsilon_{t-1}$ which allows one to observe the manner in which the magnitude and sign of returns is associated with the behaviour of return volatilities. The ability of several commonly used ARCH models to model the complex behaviour of volatilities on the Japanese stock market for the years 1980 to 1988 has been examined in this way. In order to compare the models' performance Engle and $\mathrm{Ng}$ constructed a nonparametric news impact curve for daily returns from the TOPIX index to represent the "real" impact of news on volatility. The news impact curve consisted of a simple piecewise linear function representing the response of stock returns volatility to news. Subsequently, a series of commonly used parametric volatility models was estimated on the same data, and their performance in modeling volatility was measured with respect to the baseline non-parametric model via the news impact curve. In total 6 common parametric models were estimated: $\operatorname{GARCH}(1,1)$, $\operatorname{EGARCH}(1,1)$, Asymmetric GARCH(1,1), VGARCH(1,1), Asymmetric Nonlinear GARCH( 1,1$)$, and a Glosten-Jagannathan-Runkle model. For details on these models please refer to Table 7-1 in Engle and $\mathrm{Ng}$ [Engle and $\mathrm{Ng}, 1993$ ].

Results from the non-parametric model show asymmetry: negative news causes more volatility than positive news, and "bigger" news, in general, is associated with higher volatility. The comparison of the parametric and the baseline models for different "sizes" of news $\left(\epsilon_{t-1}\right)$ yields interesting results. First, every model apart from $\operatorname{GARCH}(1,1)$ treats positive and negative news differently, the reason being that GARCH does not distinguish between the two. For "medium" size news the parametric models correctly identify the asymmetric effect between positive and negative news. The parametric models, however, seem to overestimate the effect of positive news on volatility and underestimate the effect of bad news as compared to the "true" non-parametric model. The EGARCH and GlostenJagannathan-Runkle models, however, stand out from the other models, yielding 
results which are the closest to the baseline non-parametric model.

The study raises some interesting questions. First, the use of a standard procedure to test the performance of various volatility models is a major step forwards. The use of a non-parametric technique as a baseline model for comparing parametric models, however, poses a problem: it assumes that the non-parametric model represents the "true" behaviour of volatilities. While a spline model may be more flexible, it is arbitrary, and its validity depends greatly on the number of datapoints available: caution is thus needed when comparing parametric and nonparametric models. It is interesting nonetheless to see that the EGARCH and GJR models are able to capture the more interesting aspects of volatility behaviour.

Engle, Lilien and Robins [Engle et al., 1987] examined the relationship between excess holding yields on bonds and their risk premium, measured as the excess yield's variance. The ARCH in mean model (ARCH-M) was introduced to test the hypothesis by allowing the conditional variance term to influence the mean of the process:

$$
\begin{aligned}
y_{t} & =\beta+\delta h_{t}+\epsilon_{t} \\
h_{t}^{2} & =\gamma+\alpha \sum_{\tau=1}^{4} w_{\tau} \epsilon_{t-\tau}^{2}
\end{aligned}
$$

where $\beta$ represents the mean of the process, $\delta h_{t}$ represents the time varying risk premium which affects the mean of the process through the conditional variance, and the terms $w_{\tau}$ weight past residuals.

Using quarterly bond data from 1960 to the second quarter of 1984, the authors computed the excess yield as the difference between yields on a 6 and 3 month t-bills: $y_{t}=\left[\left(1+r_{6, t}\right)^{2} /\left(1+r_{3, t+1}\right)\right]-\left(1+r_{3, t}\right)$. Different models were specified and estimated in order to test the validity of the relationship between excess yields and their conditional variance - Table 2.2 presents the results of the regressions.

The first regression performed a fit of the excess yield $y_{t}$ on a constant, giving an estimate of the average yield for the period of $0.142 \%$ per quarter. The second regression was similar for the first, with the difference that the regression errors were allowed to to follow an ARCH process of order one. The results show that the estimated average yield dropped to $0.048 \%$ once non-constant variance was allowed for. Indeed, the time series shows two distinct periods, one relatively calm, and the other with high swings in excess holding yield - allowing ARCH residuals in the model has the effect of giving the large variance observations less weight in the regression, effectively reducing the estimate of average yield. The larger log-likelihood confirms that this model is a better fit. 
Regression three represents the first attempt at fitting an ARCH-M model to excess yield data. The conditional variance variable in the estimated model has been found to be highly significant with the parameter $\delta=0.687$ expressing the time varying risk premium. The importance of this variable in the regression signifies that excess yields are indeed affected by their own past volatility. The value of the parameter $\alpha=1.64$ in the conditional variance equation implies that the unconditional variance of the excess yield is in fact infinite. The increased likelihood of this model over previous specifications is proof of its good fit. Model four is a slight modification of model three, where the conditional variance term in the mean equation is replaced with a term in the log of the variance as follows: $y_{t}=\beta+\delta \log \left(h_{t}\right)+\epsilon_{t}$. This specification has been selected through a trial-anderror process where several alternative formulations were attempted. Table 2.2 shows the results of the regression, and shows $\delta=0.135$ to be significant, together with an increased likelihood over the previous model. Finally, regression five extends the model further by adding the yield spread between the six and three month t-bills as an explanatory variable for the behaviour of excess yield. The new model mean equation is: $y_{t}=\beta+\delta \log \left(h_{t}\right)+\phi\left(r_{6, t}-r_{3, t}\right)+\epsilon_{t}$. The model again yields a higher likelihood, finding the yield spread variable to be significant with $\phi=0.392$.

While the results of the regressions seem to indicate a good fit and a fair explanatory power, there seem to be several problems with the results. Starting with model three, the unconditional variance was found to be infinite leading to a conclusion that the excess yield is generated from a fat tailed distribution. As for the fourth model, the parameter $\alpha=-1.48$ in the conditional variance equation is reported to be negative. If this is indeed true, then the model is invalid since for the variance to retain positivity the conditional variance parameters are required to be positive as well. Additionally, the mean excess yield seems to change from -0.241 to 0.355 in this model leading to further questions about the model's real performance. Finally, with respect to model five, all parameters of the model are reported to be significant, however, regression five reports $\alpha=1.64$ while regression four reports $\alpha=-1.48$. Such a sudden jump in this parameter because of the inclusion of an additional variable in the mean equation seems unlikely, particularly given the parameter's negativity in the previous regression. The increase in the likelihood for model five is also relatively small as compared to model four. The large jumps in parameter values between models three, four and five, given the rather small increases in the corresponding log-likelihoods, seem to suggest a problem with the results as such even though the evidence that excess yields are influenced by risk premia is intriguing. 
French, Schwert and Stambaugh [French et al., 1987] examined the relationship between expected stock returns and stock market volatility, and in particular the predictive power of the ex-ante measures of stock market volatility on risk premiums. The study uses two methodologies to estimate the hypothesised relationship: the first one proposes the use of ARMA like techniques to model the behaviour of stock market volatility while the second one proposes the use of the Engle, Lilien and Robins' [Engle et al., 1987] GARCH-M model.

The first methodology consists of modeling the standard volatility of the stock market with the ARMA methodology. Using daily data for the SP500 index from January 1928 to December 1984, French, Schwert and Stambaugh computed "real" monthly values of stock market volatility by estimating intra-month variance of returns for every month in the sample. An ARMA fit to this artificial dataset resulted in a time series model for the behaviour of the stock market variance. Denoting the computed monthly market volatility by $\sigma_{m, t}$, the ARMA model fit to the data is:

$$
(1-L) \ln \sigma_{m, t}=\theta_{0}+\left(1-\theta_{1} L-\theta_{2} L^{2}-\theta_{3} L^{3}\right) u_{t},
$$

where $L$ represents the "lag" operator. The fit for the whole period of data resulted in a rather poor $R^{2}=0.238$.

The core test of the article is performed by regressing excess returns on the NYSE CRSP stock market index on the expected future variance computed from the previously estimated ARMA model. The excess retum is defined as the difference between the monthly NYSE-CRSP return and the one month t-bill: $r_{m, t}-r_{f, t}$. Predicted values of market volatility $\hat{\sigma}_{m, t}^{p}$ and the values unexpected or residual volatility $\hat{\sigma}_{m, t}^{u}$ (the difference between real and predicted volatility) are computed for each month in the dataset and two regressions are computed: 1) a regression of excess returns on predicted volatility alone, and, 2) a regression of expected returns on predicted and unpredicted volatilities:

$$
\begin{aligned}
& \left(r_{m, t}-r_{f, t}\right)=\alpha+\beta \hat{\sigma}_{m, t}^{p}+\epsilon_{t}, \\
& \left(r_{m, t}-r_{f, t}\right)=\alpha+\beta \hat{\sigma}_{m, t}^{p}+\gamma \hat{\sigma}_{m, t}^{u}+\epsilon_{t} .
\end{aligned}
$$

The results of the regressions are presented in Table 2.3. The results from regression 1 suggest that there is little relation between excess returns and the predicted component of stock market variance, the parameters $\beta$ being close to zero and non-significant. On the other hand, the second regression shows there to be a significant relation between the excess returns and the unpredicted component of stock market volatility. The authors also performed similar regressions with 
variance terms instead of standard deviations, however, those yielded even poorer results.

The second methodology calls for the use of GARCH-M models in order to measure the relationship between excess returns and stock market volatility, the advantage of this technique being that it estimates the variance process at the same time as the mean process. The GARCH-M model is estimated on monthly data and has the following form:

$$
\begin{aligned}
\left(r_{m, t}-r_{f, t}\right) & =\alpha+\beta \sigma_{t}+\epsilon_{t}-\theta \epsilon_{t-1} \\
\sigma_{t}^{2} & =a+b \sigma_{t-1}^{2}+c_{1} \epsilon_{t-1}^{2}+c_{2} \epsilon_{t-2}^{2} .
\end{aligned}
$$

The model suggests that there exists a good significant and positive relationship between the standard deviation of returns and excess stock returns much more so than in the previously reported ARMA model. This discrepancy has been explained by the authors as a problem of statistical specification. Table 2.4 shows the results of the GARCH-M model.

The result of the study, that is the positive relation between the predicted component of market volatility and the excess returns, push the authors to conclude that this positive dependence of excess returns on predicted volatility results in a negative relation between expected returns and the unexpected component of volatility. The authors explain this phenomenon by the fact that "a positive unexpected change in volatility increases future expected risk premiums and lowers current stock prices".

The article's interesting results tend, however, to hide several problems. First, the ARMA models use a monthly dataset manufactured from intra-month observations. Such artificial datasets may create synthetic artifacts in the data, such as autocorrelation. Second, the study is not really predictive in nature since all computations and "predictions" are performed in-sample. This yields two important observations: 1) since the study is "in-sample" the results of the study are surely over-inflated (the fit and predictions are exaggerated), and, 2) since the study results as expressed in $R^{2}$ measures are relatively weak as it is ( $R^{2}$ values under 0.3 ), then potential "out-of-sample" benefits of the study may be even more limited. Thirdly, the weakness in the methodology and the contradictory results between the two methods used in the article seem to indicate that the results should be interpreted with caution - after all the authors add at the end of the article a comment about some additional results: "We have done some work along these lines, but the results are so ambiguous [...] that they are not worth reporting in detail". 
Bollerslev, Engle and Woolridge [Bollerslev et al., 1988] apply the GARCHM framework to the Capital Asset Pricing Model. The CAPM specifies that an asset's returns are proportional to its non-diversifiable risk which is assumed constant over time - the GARCH-M model, through its ability to model variances, makes it possible to dispense with this assumption. Using quarterly t-bill, $t$-bond and SP500 returns for the period 1959-1984, the authors proposed to model the time varying conditional variances and covariances to better explain the behaviour of stock returns.

The results show that the covariance matrix for asset returns is not constant over time and tends to vary quite significantly. As expected, the beta estimates for the market index tend to oscillate around 1 , and returns are quite well explained by the risk premia. While these results are interesting, the study seems exploratory in nature and does not attempt to explain the behaviour of stock returns with the help of other explanatory variables. A more complete study could have been conducted in order to find more exactly the determinants of stock returns.

The results from the ARCH literature show that returns have an even more complex behaviour than previously thought. The volatility of returns has been found to evolve through time, and is now known to respond positively with respect to the magnitude of "news". Additionally, volatility responds in an asymmetric manner to bad and good news, "bad" news causing greater volatility than "good" news. These results, in hindsight, are intuitive, and have a certain elegance. Unfortunately, while these general results seem sensible, questions should be asked about the reliability of individual empirical results. The reason behind this caution is the reliability of software used to implement ARCH type models. Recently, Brooks, Burke and Persand [Brooks et al., 2001] tested the ability of nine commercial software packages to estimate the simple $\operatorname{GARCH}(1,1)$.

General results showed that no two packages yielded similar model parameter estimates. Test statistics results also varied across packages, the greatest differences being observed with respect to t-statistics: a given model parameter could be construed to be significant using one software package and insignificant using another. Such a variety of results stemming from important commercial packages puts in doubt the empirical results from many volatility studies and since most studies do not report the software or algorithms used in obtaining empirical results, the absolute correctness of results should be questioned. 


\subsection{Investor Behaviour}

Historically, the finance literature has concentrated on analyzing and explaining the behaviour of financial prices. Recently, however, researchers have broadened their investigations to include analysis of investor behaviour. In particular, the efforts have centered on studying investor behaviour in order to better explain some of the anomalies present in stock prices.

The literature features numerous examples of phenomena which seem inconsistent with the concept of market efficiency and investor rationality. Some studies have found speculative market returns to be correlated through time, and some others have found that investors tend to over-react to information. The traditional finance framework has a great deal of trouble in explaining these findings.

In order to explain the various anomalies a new branch of finance has appeared which combines economic theory with insights from psychology. Thaler [Thaler, 1999] proposes that behavioral finance is a necessary step in the evolution of financial thought because it can "enrich our understanding of financial markets by adding a human element". More particularly, the new direction is needed because studying the manner in which financial markets aggregate investor behaviour can give new answers concerning market efficiency.

The theory of stock market prices, starting with Bachelier [Bachelier, 1900], states that stock prices follow random walks and are unpredictable (see Samuelson [Samuelson, 1973]). Samuelson [Samuelson, 1965] further proved that if investors use all information available to them in formulating prices, then stock market prices will follow a martingale process. Various empirical investigations of stock prices and returns have found, however, that price behaviour may diverge from this ideal.

Stock return dependence is one of the most interesting and important issues in finance. The efficient markets hypothesis stipulates that it is impossible to predict the future behaviour of returns using using historical returns data. It says that, if markets are efficient, then prices should follow a martingale process, and thus should be unpredictable. The literature features numerous dependence tests, but the results are often contradictory and open to discussion.

In one of the earliest dependence tests, Fama [Fama, 1965] computed the serial correlation of daily stock returns (for lags up to 30 ) as well as the first order serial correlation for 4,9 and 16 day retums for the 30 biggest firms at the time. While the results indicated that 11 out of 30 of the first order correlations for daily returns were significant the correlations were smaller than 0.123 in absolute value. Most one day correlations were also found to be positive. For multi-day returns, 
Fama found that, the 4-day and 9-day returns correlation was mostly negative and not significant, while the 16-day return correlation was mostly positive. Based on these results, Fama concluded that while the returns seem to display some dependence, the significance of that anomaly was weak and did not represent a profit making opportunity for the potential investor.

More recently, Lo and MacKinlay [Lo and MacKinlay, 1988] have performed another daily stock returns serial dependence test. Their goal was to provide a definite answer to the question of stock returns dependence. Compared to other studies of the kind, the strength of their analysis lay in the use of a random walk test robust to both heteroscedasticity and non-normality. Results show that the weekly returns on the equal and value weighted CRSP indices are positively and significantly correlated (correlations 0.30 and 0.08 respectively) while 4-week return correlations are not significant. Furthermore, size portfolio returns were shown to be significantly correlated with one-week returns on the small-size-firm portfolio having the largest autocorrelation $(0.42)$ and the large-size-firm portfolio returns having the smallest $(0.14)$. On the other hand, results for individual stocks show them to be negatively, but weakly correlated. These results have led Lo and MacKinlay to reject the random walk hypothesis for stock returns.

Cutler, Poterba and Summers [Cutler et al., 1991] have examined the predictability of several groups of market traded assets such as international stock indices, bonds, currency, gold, houses, and collectibles with the goal of finding whether market traded goods display similar behaviour. Using monthly data, the authors find that the serial correlation of returns on the vast majority of assets is positive and significant at horizons of less than 12 months. On the other hand, the longer lag serial correlations (between 13 and 24 months) are, on the average, negative. For example, the average first lag autocorrelation of the international stock indices is 0.1 , while for bonds it is 0.4 , and for exchange rates the correlation is 0.067 . Concerning housing, farms, and collectibles, the authors find yearly returns on these assets to be positively correlated as well $(0.2,0.727$, and 0.365 respectively). The authors conclude that the common pattern in returns displayed by the various assets can only be a result of the speculative process resulting from an open market.

The correlation trends in stock returns have lead some researchers to propose the "mean reversion hypothesis". The short-term positive and long-term negative correlations suggest that prices tend to diverge from fundamental values, but come back to them after a certain period of time. This phenomenon in effect says that the market makes errors in estimates of value, but tends to correct them over time.

Poterba and Summers [Poterba and Summers, 1988], using CRSP monthly 
returns, for the period 1926-1985 have found that variance ratios indicate positive autocorrelations for up to 24-month returns, and negative autocorrelations for longer period returns. Similarly, using an extended dataset ranging from $1872-$ 1985, the authors find a slightly weaker pattern. Further enhancing the study, the authors examined index returns for 17 foreign countries: while the patterns for the different markets were similar to those observed in the US market, the test lacked power to reject the random walk hypothesis.

Fama and French [Fama and French, 1988a] have also examined the phenomenon, by studying correlation patterns of CRSP size and industry sorted portfolios for the period 1926-1985. According to their model, autocorrelations should display a u-shape with respect to the returns horizon: for short term returns, correlations should be close to 0 , for medium term returns, they should be negative, and for longer terms, they should converge back to 0 . The results have shown industry portfolios to display the hypothesized $u$-shape, correlations being greatest and negative for horizons of 3,4 and 5 years: on average, $-0.38,-0.45$ and -0.45 respectively. Size decile results have shown a similar pattern, however with smallsize firms displaying higher autocorrelations at medium-term intervals. Fama and French concluded that this observed pattern could potentially suggest market inefficiencies.

Kim, Nelson and Startz [Kim et al., 1991] found that the evidence in support of mean reversion varies over time. The authors have found that the correlation of returns has a different structure before and after the Second World War period. The familiar mean reversion characteristics of positive short-term correlations and negative long-term return correlations seems only to exist before 1947 and disappears after 1947. The u-shape pattern of correlations proposed by Fama and French [Fama and French, 1988a] seems to be characteristic of the period 19261946 only. While the full period 1926-1986 also displays this pattern, it seems to be a statistical artifact of the pre-war period. An analysis of post-war data indicates that the pattern is not present. Instead, the authors have found correlations to persist over time in the post-war period: correlations appear to be positive at all lags for the years 1947-1986.

The puzzle of positive first order autocorrelation of stock index returns and the negative first order autocorrelation of individual stock returns has intrigued many researchers. Lo and MacKinlay [Lo and Mackinlay, 1990] have proposed a simple but elegant answer to this question. They proposed that, since an index is simply a collection individual stocks, positive index correlation can be explained by studying the nature of the auto-covariance function. The indice's auto-covariance is a sum of individual stock auto-covariances and the individ- 
ual stocks' cross-auto-covariances: empirical results have shown that index autocovariances are positive, and stock auto-covariances are negative - therefore the cross-auto-covariances between individual securities must be relatively large and positive for index correlations to be positive. Based on this deduction the authors have posited that the so-called contrarian strategies owe their success to the correlation across securities, and not to predictable patterns in individual securities' returns. Lo and MacKinlay have found stocks to be positively cross-correlated in time and that around $50 \%$ of profits from contrarian strategies can be attributable to cross-correlation.

The psychology literature has discovered that in making decisions individuals tend to over-react by giving recent information too much weight and discounting past information too quickly. DeBondt and Thaler [DeBondt and Thaler, 1985] proposed to test the hypothesis of whether this finding also applies to the financial markets. In their study the authors examined the behaviour of badly performing stocks versus that of well performing stocks. If the over-reaction hypothesis were to hold, the authors would find that a strategy of selling short the well performing stocks and buying badly performing stocks would yield positive abnormal returns. The reason behind this is that investors' over-reactions would push the prices of well performing stocks above, and that of badly performing stocks well below, fundamental values forcing the prices to reverse their course towards fundamental values after a certain time. The study found that the "loser" portfolio recorded an average profit of $19.6 \%$ and that the "winner" portfolio recorded a loss of $5 \%$ for a total return of $24.6 \%$. Furthermore, the study found that most returns were generated in January. The findings thus confirmed the hypothesis and demonstrated the fact that investors have a tendency to over-react to incoming information.

The question whether investors are rational centers on the issue of whether they are able to correctly interpret information. Daniel and Titman

[Daniel and Titman, 1999] say that investors cannot process the vast quantities of information available to them, and that the information is often too imprecise for them to form good quality estimates. The scarcity of quality data causes individuals to give too much emphasis to their own sources of information and thus overestimate its accuracy. One implication is that investors will be overconfident about firms on which only subjective information is available, and will tend to be more on the mark with respect to firms for which plenty of clear information is available (for example new-economy Internet stocks vs. old-economy stocks).

Such over-confidence will generate momentum in stock prices. In order to verify their assertion the authors tested the existence of the momentum effect by examining stocks on the basis of their book-to-market ratio. Investors are hypothe- 
sized to be more overconfident about stocks with low $B / M$ ratios because the value of such stocks is more difficult to evaluate since such companies are more dependent on intangible assets and growth (for example high-tech companies). The results suggest that the momentum effect is greatest for low B/M stocks. Trading rule tests showed that the average excess profit from exploiting the momentum effect was $1.04 \%$ per month, or $12.64 \%$ per year.

Because it is difficult to interpret and explain some of these findings in the standard rational investor framework, a new branch of finance, "behavioural innance", has appeared [Thaler, 1999] to attempt to explain these phenomena. The novelty of this literature consists in attempting to explain the observed price behaviours through models of investor behaviour rooted in psychology. Abandoning the "absolute investor rationality" credo, the literature instead proposes to explain the various financial anomalies through well established investor behaviours. Daniel et al [Daniel et al., 1998] say on this topic that "a good psychological finance theory will be grounded on psychological evidence about how people actually behave".

Daniel, Hirshleifer and Subrahmanyam [Daniel et al., 1998] hypothesized that the observed phenomena of over and under-reaction are caused by investor overconfidence and biased self attribution. Investors become overconfident about the accuracy of the data

Over-confidence is the erroneous assessment of the accuracy of the information the investor has access to and occurs mostly in the cases where the information is private. This applies mostly to individuals such as analysts who tend to discover information themselves, and who can potentially overrate their own expertise. Biased attribution on the other hand affects those investors who are already overconfident about their information. This phenomenon occurs when public information becomes available which confirms the investors' decisions, decisions taken on the basis of their own private information (research). Then, the arrival of information confirming previous decisions can potentially further raise investors' confidence in their own skills while the arrival of contrary information will not alter investors' self perception of their analytical abilities.

According to the model investors overreact to private information and underreact to public information. The implications are that securities tend to overreact to news in the short term, and to correct in the long term, causing short-term positive and long-term negative autocorrelations as found in the empirical literature.

Hong and Stein [Hong and Stein, 1999] proposed another approach to explain the phenomena of under and overreaction. While the Daniel, Hirshleifer and Subrahmanyam [Daniel et al., 1998] paper hypothesized that these phenomena are 
caused by innate investor characteristics, Hong and Stein proposed instead that they are the result of interaction among investors in the marketplace. The model supposes two types of traders: "news-watchers" who only trade on the basis of news, which tends to diffuse slowly, and cannot look at historical price data, and "momentum traders" who look at the history of prices exclusively.

While news-watchers trade on the basis of news, momentum traders trade on the basis of recent price changes. The slow diffusion of information among informed traders causes the prices to underreact. This enables trend-chasers to purchase stocks and thus drive the prices upwards causing them to overreact. Hong and Stein show that momentum traders in this framework can be successful and make money in the situation when they catch the rising price trend fast enough. To quote Hong and Stein "the very existence of underreaction shows the seeds for overreaction, by making it profitable for momentum traders to enter the market". When the news diffuses fully among the news-watcher population the prices are corrected. This then causes the observed negative and positive autocorrelations.

Lately, Hong, Lim and Stein [Hong et al., 2000] tested Hong and Stein's [Hong and Stein, 1999] model of under and overreaction. The question asked by the investigators was whether the gradual diffusion of information to newswatchers would generate momentum in prices. The underreaction hypothesis stated that in slower diffusing stocks momentum would be greater. In order to test the hypothesis the authors studied the behaviour of prices as a function of firm size and analyst coverage with the idea that information concerning smaller and less reviewed firms would be slower to propagate across the market causing underreaction. The results show that momentum is an inverse function of firm size: the larger the firm, the faster news is transmitted, and the smaller the momentum. The same has been discovered with respect to analyst coverage. These results seem to confirm Hong and Stein's [Hong and Stein, 1999] original model.

Informational cascades are another possible explanation for momentums and reversals. Bikhchandani, Hirshleifer and Welch [Bikhchandani et al., 1992] suggest that in cases when information is private and investors can only learn information possessed by others by observing their actions it is possible that such investors will ignore the information they themselves own and imitate the behaviour of their predecessors. In such case an investor who does not have any private information may simply imitate the action taken by another investor. If other investors follow suit an "informational cascade" will be created where individuals ignore their own information and simply imitate the behaviours of others. Such a cascade could potentially explain the momentum phenomenon. A cascade can be broken or interrupted when information concerning the value of the underlying 
asset becomes available - in such a case, a reversal could occur.

Another interesting financial mystery is the question of the large equity premium. The problem refers to the very high difference between the average returns on stocks (approx. 7\% per year) and risk-free securities (approx. 1\% per year). While investor risk aversion can explain some of the premium, it is difficult to attribute the whole difference to the risk premium alone. Benartzi and Thaler [Benartzi and Thaler, 1995] have proposed a behavioral explanation to the excessively high observed premium. The explanation centers on two psychological observations: loss aversion, and mental accounting. Individuals are loss averse when their dislike of losing wealth is greater than their preference for gaining the same amount of wealth. Mental accounting on the other hand refers to the fact that investors evaluate various investments differently.

Benartzi and Thaler proposed that myopic risk aversion can explain the observed equity premium. Myopic risk aversion refers to the fact that investors are more willing to invest into risky assets when the investment's time horizon is long, and when they do not evaluate the investment's performance frequently. The authors' goal was to find the optimal investment evaluation frequency as well as the optimal mix of stock and bonds in investor portfolios in order to explain the observed equity premium. The results showed that in order to explain the observed risk premium investors evaluate their investments approximately every twelve months, and that their allocation of stocks and bonds was about equal $(50-50)$.

The results show that investors require the high equity premium in order to justify their investments in stocks. To quote Benartzi and Thaler: "for investors who must account for near term losses, these long term results may have little significance". In other words, because investment managers may be victims of the high riskiness of equities in the short-term they require a high expected return. 


\begin{tabular}{lccccc}
\hline Model & Const. & $\delta$ & $\gamma$ & $\alpha$ & LL \\
\hline Reg. 1 & 0.142 & & & & 51.1 \\
Reg. 2 & 0.048 & & 0.004 & 1.9 & 85.17 \\
Reg. 3 & -0.241 & $0.687^{*} h_{t}$ & 0.0023 & 1.64 & 96.34 \\
Reg. 4 & 0.355 & $0.135^{*} \log \left(h_{t}\right)$ & 0.005 & -1.48 & 101.35 \\
Reg. 5 & 0.325 & $0.130^{*} \log \left(h_{t}\right)$ & 0.004 & 1.64 & 103.48 \\
\hline \hline
\end{tabular}

Table 2.2: Engle, Lilien and Robins: ARCH-M models [Engle et al., 1987]

\begin{tabular}{lcccc}
\hline Regression & $\alpha$ & $\beta$ & $\gamma$ & $R^{2}$ \\
\hline Reg. 1 & 0.0047 & 0.0023 & & $\mathrm{n} / \mathrm{a}$ \\
Reg. 2 & 0.0077 & -0.050 & -1.010 & 0.152 \\
\hline \hline
\end{tabular}

Table 2.3: French et al. excess return regressions on ARMA predicted volatilities [French et al., 1987]

\begin{tabular}{ccccccc}
\hline$\alpha$ & $\beta$ & $a \times 10^{3}$ & $b$ & $c_{1}$ & $c_{2}$ & $\theta$ \\
\hline-0.0020 & 0.224 & 0.083 & 0.814 & 0.058 & 0.104 & -0.073 \\
\hline \hline
\end{tabular}

Table 2.4: French et al. monthly excess return GARCH-M model results [French et al., 1987] 


\section{Chapter 3}

\section{Models of Returns}

Studies of market predictability can be divided into three categories: explanatory regressions, time series models and trading rules. The first two categories represent an attempt at the development of statistical models to capture the behaviour of returns and to predict their future course. Regression models mainly use economic and technical variables to predict the future behaviour of returns, while time series models attempt to model the temporal behaviour of returns to forecast their behaviour. Technical analysis, on the other hand, is a collection of ad-hoc rules developed by market participants over time designed to help them predict the course of the market. Recent studies have found that not only can technical analysis forecast returns, but that it is also related to statistical methods. The ARCH literature is not included in this section since it mostly consists of in-sample studies and is therefore not of a truly predictive nature. The chapter is divided into three parts: it starts with a review of predictive regression models, follows with the time-series literature analysis, and finally, looks at studies of technical analysis methods.

\subsection{Regression Models}

Chan, Kacerski and Lakonishok [Chan et al., 1998] examined the ability of 5 classes of factors frequently used in the literature to distinguish between well and badly performing stocks: 1) fundamental factors, or stock dependent variables such as accounting ratios, 2) technical factors, based on the technical analysis literature, 3) economic factors, 4) statistical factors extracted through principal components methods, and 5) the market factor, in the form of the equally weighted and 


\begin{tabular}{lcccc}
\hline Factor & Avg. Return & St. Dev. & January Return & St. Dev. \\
\hline Book/Market & 0.0059 & 0.0379 & 0.0646 & 0.0554 \\
Dividend Yield & 0.0008 & 0.0372 & 0.0173 & 0.0355 \\
Size & -0.0034 & 0.0511 & -0.0854 & 0.057 \\
$R_{t, t=[-7,-1]}$ & 0.0064 & 0.0416 & -0.0561 & 0.0727 \\
$R_{t, t=[-60,-12]}$ & -0.0046 & 0.0415 & -0.0640 & 0.0738 \\
$R_{t, t=[-1,0]}$ & -0.0179 & 0.0375 & -0.0722 & 0.0777 \\
Default Prem. & 0.0019 & 0.0297 & -0.0403 & 0.0497 \\
Term Prem. & 0.0005 & 0.0339 & -0.0299 & 0.0465 \\
Value wtd. Factor & -0.0020 & 0.0469 & 0.0422 & 0.0707 \\
Equal wtd. Factor & 0.0005 & 0.0570 & 0.0697 & 0.0871 \\
\hline \hline
\end{tabular}

Table 3.1: Chan, Kacerski and Lakonishok [Chan et al., 1998] mimicking portfolio return statistics.

value weighted return on the CRSP index.

The mimicking portfolio methodology was used consisting of forming quintile portfolios by sorting stocks according to their loading on each factor every April, and then computing mimicking portfolio predictive returns as the difference in returns between the first and fifth quintile portfolios for a subsequent 12 month period. Factor importance analysis was conducted by analyzing the mean and variance of the mimicking portfolio returns with the factors found to be the most important being presented in Table 3.1.

The analysis of the variance of mimicking portfolio returns uncovered that few of the fundamental or economic factors could explain the covariation in returns: only size, book to market, and dividend yield factors were found to be the best discriminators from among the fundamental factors, and the default and term yields from among macro-economic factors. The technical factors were surprisingly good descriptors of returns as were the market factors. The mimicking portfolio average returns showed that the spread between the high and low factor loading stocks were relatively small for all factors. The return spread differed significantly, however, between up and down market months: fundamental factor returns were especially interesting, being consistently positive during down market periods. Chan, Kacerski and Lakonishok interpreted this finding as the proof of a "flight to quality" among investors during bad market conditions: investors tended to prefer stocks with safe fundamentals in these cases.

Further analysis has shown the January effect to be very important. Analyzing returns from portfolios for the month of January, it has been found that the January 
returns were the highest and December retums the lowest for the whole year (see Table 3.1). The retum variance was also found to be slightly higher in January reflecting the increased mean return in that month. The authors have related this result to the end of year "window dressing" phenomenon when fund managers tend to sell under-performers and purchase safer stocks. Strategies based on technical factors tend, however, to amass negative returns at the beginning of the year, and positive returns at the end of the year.

In conclusion, the performance of most factors has been found to be very poor: the factors used in the literature are unable to explain the variations in returns. The most important finding has been the persistence of the January effect across all factors. It seems that investors like to re-balance portfolios at the end of the fiscal year, and invest in stronger companies, and then buy the weakened stocks in the new year. The dividend yield has also been found to be important as portfolios based on it have been able to perform well during economic downturns.

Rozeff [Rozeff, 1974] explored the relationship between stock returns and monetary variables, and in particular, the effects on stock returns of lagged changes in monetary policy. He elaborated two models to test market efficiency vis-a-vis monetary policy changes. The first model, the predictive model, assumed that stock returns can be predicted using monetary variables while the second model, the non-predictive model, assumed that changes in monetary variables affect only the current returns. The first model described stock returns exclusively as a function of lagged monetary variables, and the second model described them as a function of concurrent and future monetary variables arguing that investors not only incorporate all information in current prices but also anticipate future changes in monetary policy.

To test the response of returns to new monetary information Rozeff developed a univariate model describing stock movements as a function of second order changes in the money supply:

$$
R_{t}=c_{0}+\sum_{i=1}^{J} a_{t-i}\left(g_{t-i}-g_{t-i-1}\right)+\epsilon_{t}
$$

where $g_{t}$ is the change in the money supply $M_{t} / M_{t-1}$ and $c_{0}, a_{t-i}$ are parameters. The predictive and non-predictive models were distinguished by varying the range over which changes in the money supply were included in the model.

Rozeff conducted three separate tests: a purely predictive test, in which only past variables were used to forecast future returns, an explanatory test, where past and current variables were used, and finally a non-predictive test where past, 


\begin{tabular}{llcc}
\hline Type & Model & Period & $R_{a d j}^{2}$ \\
\hline predictive & $R_{t}=c_{0}+\sum_{i=1}^{I 7} a_{t-i}\left(g_{t-i}-g_{t-i-1}\right)+\epsilon_{t}$ & $1948-1970$ & 0.018 \\
& & $1948-1960$ & 0.100 \\
& & $1960-1970$ & 0.001 \\
\hline explanatory & $R_{t}=c_{0}+\sum_{i=0}^{I 7} a_{t-i}\left(g_{t-i}-g_{t-i-1}\right)+\epsilon_{t}$ & $1948-1970$ & 0.054 \\
& & $1948-1960$ & 0.207 \\
& & $1960-1970$ & -0.018 \\
\hline non-predictive & $R_{t}=c_{0}+\sum_{i=-8}^{I T} a_{t-i}\left(g_{t-i}-g_{t-i-1}\right)+\epsilon_{t}$ & $1948-1970$ & 0.154 \\
& & $1948-1960$ & 0.196 \\
& & $1960-1970$ & 0.168 \\
\hline \hline
\end{tabular}

Table 3.2: Rozeff's [Rozeff, 1974] regression results

present and current explanatory variables were included in the model. The study was conducted on monthly returns on the SP500 index for the period 1926-1970 and the M1 money supply variable from the Federal Reserve Board for the same period. Table 3.2 presents the principal results for the study:

The results indicate that the market is efficient with respect to information contained in monetary variables. The predictive models, using only past monetary variables to forecast returns is unable to yield respectable $R^{2}$ values. Interestingly, the model works rather well in the first sub-period, but its power deteriorates in the subsequent period. The explanatory model, including a concurrent monetary variable has additional power over the simple predictive models for the overall period, but its advantage is small. Non-predictive models on the other hand fare quite well with $R^{2}$ close to 0.2 , and behave more evenly during the two subperiods.

Model profitability was then tested using a trading rule. The trading rule consisted of investing in the market portfolio when the predicted return was higher than the current month's risk free rate, and in bonds in the opposite case. A rolling regression was used to update predictive model coefficients every month. The predictive model's profits over the 1956-1970 period averaged $3 \%$ per year, compared to $7 \%$ for the buy-and-hold strategy, and $12 \%$ for a model using the current month's data, after $1 \%$ transaction costs.

Fama and Schwert [Fama and Schwert, 1977] found that stocks are not good hedges against inflation. They examined, among others, the relationship between equal and value weighted portfolios of stocks from the CRSP database for the period 1953-1971. Using the one month t-bill rate, $B_{t}$, as a measure of expected 
inflation, Fama and Schwert estimated a stock's sensitivity to expected and unexpected inflation using following model:

$$
R_{t}=\alpha+\beta B_{t}+\gamma\left(\Delta_{t}-B_{t}\right)+\epsilon_{t}
$$

where, $\Delta_{t}$ is the realized inflation rate, and $\left(\Delta_{t}-B_{t}\right)$ the unexpected inflation component. Regression results reject the hypothesis that both the value and equally weighted stock portfolios are hedges against expected inflation: the $\beta$ coefficients for both assets were -5.5 and -5.7 respectively, instead of $\beta=1$ which characterizes a perfect hedge. The results also showed that stocks are not good hedges against unexpected inflation with $\gamma$ coefficients of -0.77 and -2.35 for the value and equally weighted portfolios respectively.

The authors further examined the relationship between stock and inflation through a trading rule. The trading rule, similar to the one used by Rozeff, used a rolling regression of the value weighted portfolio return on the one month $t$-bill to forecast the next month's expected return. If the predicted return was smaller than the month's t-bill rate, bonds were held, and otherwise money was invested in stocks. The trading rule returns on the value weighted portfolio were found to be $8.4 \%$ per year compared to $7.1 \%$ and $4 \%$ for the buy-and-hold and bond holding strategies respectively. After adjusting for $1 \%$ trading costs, the profitability of the trading rule fell, however, to $6.2 \%$.

Campbell [Campbell, 1987] examined the relationship between term structure variables and stock returns. Using data for 1959-1979 and 1979-1983, he regressed monthly returns on beginning of month term structure data comprised of the one month $t$-bill rate, the beginning of month and the one period lag of spread between the two and one month t-bill rates, and the six month minus one month rate. The $R^{2}$ for the two periods was 0.112 and 0.228 respectively. The one month t-bill rate was found to be the most important predictor, its negative coefficient in both periods indicating that high interest rates lead to lower returns the following month. Campbell next examined the relation of predictor variables to return variance by regressing squared residuals on the 4 predictors. The most significant variable was found to be the one month t-bill return, with a positive relation to the return variance. Campbell also found a negative relation between stock returns and their variance. This finding concurs with the evidence - a negative relation between returns and the one month bill rate at the beginning of the month, and a positive effect between the one month bill rate and return variances, yields in sum a negative relation between returns and their variance.

Keim and Stambaugh [Keim and Stambaugh, 1986] tested the univariate predictability of risk premiums on NYSE size quintiles and various bonds with re- 
spect to three factors identified on the basis of their relationship with asset prices. The three variables were: 1) UBAA: yield difference between under-BAA rated corporate bonds and one month t-bills, 2) SP: the ratio of the SP500 level to it's 45-year moving average, and 3) PQ1: minus the average of the logarithms of prices for the smallest stock quintile.

Using monthly data for the period 1928-1978, the authors estimated a linear model of the form $R_{t}=a_{0}+a_{1} X_{t-1}+\epsilon_{t}$, where $R_{t}$ was the excess stock return for month $t$ and $X_{t-1}$ one of the three regressors measured at time $t-1$, for each of the three explanatory variables. Preliminary results showed that the relationship between explanatory variables and stock returns is positive but weak, with coefficients for all portfolios and variables having t-values ranging from 1.16 to 2.27 . Results for the 1928-1952, and 1953-1978 sub-periods showed the same signifcance patterns, but regression coefficients were found to be negative for small size portfolios for the latter period. Overall, the $R^{2}$ were very low: almost all were below 0.01 indicating that the explanatory power of the regressions was small.

Tests for a January effect in the response of stocks to the PQ1 variable performed with a dummy regression showed that the variable's importance increases in the month of January: the non-January regression coefficients have been found to be insignificant, in contrast to January coefficients. Interestingly, the January coefficients were the most significant for the small stock portfolios indicating a strong January effect in small stocks. The $R^{2}$ statistics improved with the inclusion of dummy variables reaching up to 0.13 for the smallest stock portfolio for the overall 1928-78 period. Sub-period results for small stock portfolios were encouraging as well, with $R^{2}>0.12$ for the smallest stocks for both the 19281952 and 1953-1978 sub-periods. The $R^{2}$ for the large stock portfolios remained very low even with the inclusion of January dummies: the statistic never exceeded 0.006 . An additional regression of the return difference between the smallest and largest stock portfolios on January dummies and the PQ1 variable yielded a surprisingly large $R^{2}$ of 0.23 . Variable significance was, however, unchanged: only January variables were found to be significant.

Keim and Stambaugh next performed one month ahead predictions over the period 1953-1978 using a univariate model in the PQ1 variable estimated over 1928-1952. The model's forecasts were compared to a naive "historical mean" model, and reductions in MSE were computed based on this benchmark. The results are intriguing. For the month of January, the univariate forecasting model achieved a reduction in mean square error compared to the historical mean model of $10.8 \%$ for the portfolio of big stocks and an impressive $41.8 \%$ for the portfolio of small sized stocks. The medium sized portfolio of stocks registered a MSE 
decrease of $16.7 \%$. For non-January months, the situation was very different: the average MSE independent of portfolio size increased on average by $6 \%$ ! This finding seems to emphasize the importance of the January anomaly, but its cause is not resolved in this paper.

Pesaran and Timmerman ([Pesaran and Timmerman, 1994], [Pesaran and Timmerman, 1995], [Pesaran and Timmerman, 1999]) published a series of innovative studies concerning returns predictability. The studies represented an attempt at predicting the returns of US and UK stock indices from the point of view of a market participant and were characterized by an innovative methodology as well as a profitability analysis in the light of trading costs.

In their first study, Pesaran and Timmerman [Pesaran and Timmerman, 1994] conducted a regression analysis to forecast SP500 and DJIA returns using variables identified in the literature as predictors of future returns. Using annual, quarterly, and monthly data for 1954-1991 (the full data set), the authors constructed one period ahead predictive models by identifying factors providing the best fit (c.f. Tables 1, 2, and, 3 in [Pesaran and Timmerman, 1994] for the selected models). All six models have the same structure; all contain the same variables, and in particular, the dividend yield (YSP, YDJ), changes in interest rates (DI) and inflation (PI). The annual model used as a variable the difference between the 6-month commercial paper yield and the 3 month t-bill rate (TERM) while the quarterly and monthly models used the change in industrial production (DIP). The signs of some explanatory variables were consistent with previous research and theory: the signs on dividend yield were positive, and the signs on inflation and interest yield spread respectively were negative.

The annual SP500 (DJA) model using four variables achieved an $R^{2}$ explanatory power of $60 \%(55 \%)$ compared to $20 \%(20 \%)$ and $8.2 \%(8.3 \%)$ for the quarterly and monthly models respectively. The high explanatory power of the annual model was certainly caused by data scarcity since only 36 annual observations were available, and, as the number of observations increased, as in the quarterly (144 obs.) and monthly ( 432 obs.) models, the explanatory power of the model dropped significantly. The authors also found that annual excess returns were normally distributed while quarterly and monthly returns were not. This could have further weakened the predictive power of the models.

To test the effects of nonlinearities the authors computed regressions including lagged squared returns and have found them to be positively related to future returns suggesting that volatility increases with stock returns. The inclusion of the variables increased the $R^{2}$ statistic for the SP 500 portfolio from $24 \%$ to $29 \%$ for the quarterly data model, and from $8 \%$ to $11 \%$ for the monthly data model. 
The authors tested the predictive power of the models using a recursive forecasting procedure consisting of re-estimating model parameters and performing one period ahead forecasts each time a new observation became available. Forecasting ability was verified with sign and trading rule out-of-sample tests. Sign tests were designed to verify whether predictive models could identify periods of positive and negative excess returns. Trading rule tests examined the profitability of the prediction rules.

Graphical examination showed that the recursive models had an ability to predict negative excess returns on stocks for annual, quarterly and monthly data. A statistical test of predictive ability found that the proportion of correct sign predictions for the SP500 and DJA indices exceeded $55 \%$ for monthly data, and $60 \%$ for quarterly data, for the 1960-1990 period, although sub-period results were not significant. Lack of annual data invalidated the annual model tests.

The trading rule consisted of buying stocks when predicted returns were positive and bonds otherwise. The trading rules were adjusted for costs arising from an active strategy. Without trading costs all trading rules outperformed the buyand-hold strategy by $1.9 \%, 2.2 \%$ and $2.3 \%$ for the annual, quarterly and monthly predictive models respectively (for the SP500). After transaction costs only the annual and quarterly strategies performed well with $1.5 \%$, and $1.1 \%$ returns over buy-and-hold, while the monthly prediction model yielded a negative $1 \%$. An interesting result concerning variance of returns was also discovered. Trading rule returns were less volatile than the market returns for all prediction horizons. This finding was confirmed by the Sharpe, Jensen and Treynor performance measures and a short analysis which showed that this tradeoff was not attainable through a randomly switching portfolio.

The study, while diligently constructed, is basically flawed. The forecasting models have been selected based on the whole data set. As such, the study is not truly predictive, but rather explanatory in nature, and the relatively high $R^{2}$ values obtained are an illusion and do not represent the true predictive power of the models. As such the study does not say anything on the predictability of stock returns, and the encouraging results obtained through the sign and trading rule tests are misleading.

In their second study, Pesaran and Timmerman [Pesaran and Timmerman, 1995] attempted to correct the basic mistake of their first paper by assuming that the individual investor does not know the exact model of returns in advance, nor its parameter values, and, instead, must find them. The author's only assumption in the new study was that the investor used a restricted and known set of predictor variables in formulating the market forecasts. Following the literature, the authors 
identified 9 predictive variables which are presented in their catalog of variables.

Using monthly SP500 returns data for 1959-1992, the authors proposed a new methodology consisting of selecting a predictive model each time new data is received. While a forecasting rule usually consists of a single predictive equation, the authors assumed that a real investor was confronted with the problem of model uncertainty. Assuming the investor's data-set was composed of 9 variables, the authors proposed that the investor, at each point in time new data is received, was faced with $512\left(2^{9}\right)$ potential forecasting models, from among which he needed to select only one to perform the best possible forecast. The methodology thus consisted of evaluating 512 rolling regressions of the form: $R_{t+1}=\beta_{i} X_{t i}+\epsilon_{t+1, i}$, where $R$ is the return in month $t+1, \beta$ the regression parameters, $X_{t, i}$ is the variable vector, and $i \in[1,512]$ is the number of the model, every month. The monthly regressions were estimated using the incremental data-set, and one period ahead forecasts were performed for a total of 202752 regressions and predictions.

In order to help the investor identify the right model at each point in time the $R^{2}$, Akaike, Schwartz, a custom sign, recursive wealth, and recursive Sharpe ratio criteria were used to select the best models from among the 512 competitors. Every selected model was then used to form a one-period-ahead prediction, and the forecasting performance across error criteria was compared. The full model containing all the variables was also evaluated.

Tests of predictive ability have rejected the null of no predictive ability for all selection criteria except for the Schwartz measure. Sub-sample analysis has shown, however, that returns were highly significant in the 1970 s, but not so in other periods. The authors have also examined the profitability of trading rules based on the different forecasts through the switching portfolio method of Pesaran and Timmerman [Pesaran and Timmerman, 1994]. It has been found that under the no-cost hypothesis, the returns for all model selection criteria exceed those of the buy-and-hold strategy with less risk. The highest mean (st.dev.) return was registered for the strategy based on the $R^{2}$ criterion of $14.83 \%(11.37 \%)$, compared to $11.39 \%(15.71 \%)$ for the buy-and-hold return. Interestingly, this and all other strategies outperformed the buy-and-hold strategy not only with respect to mean return, but also with respect to the standard deviation of returns which were all lower for the active trading rules. As the costs of trading were raised, however, the advantages of the trading rules over the buy-and-hold diminished significantly with only the Akaike, $R^{2}$ and full models outperforming it (the trading rules still outperformed the buy-and-hold with respect to the variability of returns however). Curiously, the full model has one of the highest returns of all models, but it's standard deviation is also higher indicating that the large num- 


\begin{tabular}{llcccccc}
\hline \multirow{3}{*}{ No costs } & & B\&H & Akaike & Schwartz & $R^{2}$ & Sign & Full model \\
& Mean return & 11.39 & 14.06 & 11.68 & 14.83 & 13.69 & 14.46 \\
& St. Dev. & 15.71 & 11.16 & 10.73 & 11.37 & 9.06 & 11.61 \\
& Mean Return & 11.29 & 11.48 & 7.91 & 12.13 & 10.25 & 11.78 \\
& St. Dev. & 15.73 & 11.69 & 10.95 & 11.58 & 9.40 & 11.91 \\
\hline
\end{tabular}

Table 3.3: Profitability of the model selection criteria based trading rules under No and High cost scenarios ([Pesaran and Timmerman, 1995] p. 1220)

ber of variables somehow introduces noise into predictions. Table 3.3 presents a summary of the findings.

The differences between the no-trading-cost and trading-cost scenarios were based on the number of transactions that a trading rule has called to be executed. However, even though the high transaction costs have reduced the profitability of the trading rules, the Sharpe ratio still found the active investment strategies to be more mean-variance efficient compared to buy-and-hold. Analysis of variables has shown that the most frequently selected variables are the lagged one month t-bill rate followed by the growth in money supply, and the lagged dividend yield.

Pesaran and Timmerman [Pesaran and Timmerman, 1999], in a follow-up to this study, used a similar methodology to forecast the returns on the Financial Times All Share Index. The major innovation of their new study was to compartmentalize the explanatory variable into categories: 1) focal variables present in every regression, 2) secondary focal variables from which a subset is selected at every iteration, and 3) additional variables which are included in the search only if the regression residual exceeded $3 \sigma$ (see the Catalog of Variables). This formulation avoided the complications related to estimating $2^{n}$ models, where $n=$ number of variables, at every point in time new data was received since the model search only included secondary and additional variables.

Using the same methodology as for their 1995 study, Pesaran and Timmerman found that, like in the US case, active trading strategies on the British market could outperform the buy-and-hold strategy. Even though no trading rule could exceed the mean return attained by the buy-and-hold the Sharpe ratio has shown the trading rule performance to be superior given the level of risk it carries.

These studies present a carefully designed but flawed attempt at forecasting future returns. The first study, as has already been told, is an in sample explanatory study and not a true attempt at prediction. The remaining two studies attempted to correct the first study by introducing a recursive forecasting methodology without 
help of hindsight. The search procedure they introduced is, however, significantly flawed. In the first study it takes 512 regressions every month to find the right model while in the second this number is just a bit less. Both approaches suffer from a version of the "curse of dimensionality": incorporating additional explanatory variables will make the models impractical because of the exponential growth of potential forecasting models. While the last study has attempted to solve this problem through variable classification, incorporating enough variables will break this scheme as well. While ultimately the reasons for a model search could be understood, it is interesting to ask why this approach has not been implemented in a cleaner framework such as a state space model.

In the end, such a massive model search resembles data mining more than an attempt at explaining future returns. In result, the profits gained from this approach are not much better than those for a buy-and-hold strategy. This is because the study uses variables discovered in the past to have explanatory power over future returns while the instrument it uses to forecast them is too blunt. In the end, considering the massive computer power requirements of the methodology, the studies did not succeed in explaining financial returns, and can be regarded as a failure.

Cutler, Poterba and Summers [Cutler et al., 1989] examined stock price movements in the light of quantitative macro-economic and qualitative news. The authors studied the impact of quantitative news on stock prices by examining the explanatory power of innovations in economic variables on monthly and annual stock returns from the NYSE value weighted index for the period 1926-1985 and 1871-1986 respectively. Innovations for 7 economic variables were obtained by subtracting the predicted values of economic variables obtained through a vector auto-regression from their realized values. A regression of monthly and annual returns on the extracted innovations was then performed. The results showed that innovations were not able to fully explain the behaviour of monthly or yearly stock returns: the $R_{A d j \text {. }}^{2}$ statistics averaged $18.5 \%$ and $6 \%$ for monthly and yearly regressions respectively. Sub-period results for monthly returns, yielded even lower statistics, with $R_{A d j .}^{2}=14 \%$ for the period 1946-1985. Increasing the number of lags in the vector auto-regressions to extract even more information from past economic variables did not change the quality of fit. In all regressions, interest rates, inflation, and volatility innovations were negatively related to returns, while dividends, industrial production, and money supply were positively related, however only dividends, industrial production, inflation, and volatility were significant.

The authors further examined the series of returns by using an approach similar to one used by Rozeff [Rozeff, 1974], regressing returns on past, past and 
current, and past, present and future explanatory variables. For monthly data, using only lagged variables, the $R_{\text {Adj. }}^{2}$ statistic has values less than $5 \%$, while using lagged and contemporaneous variables the statistic ranges from $13 \%-30 \%$ depending on the number of lags in the regression. Regressions with past, present, and future explanatory variables, on the other hand, explain returns very well with

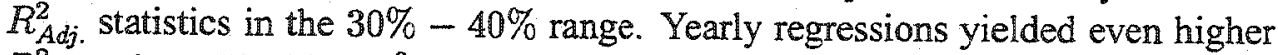

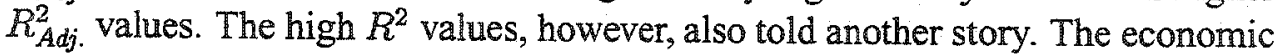
variables examined could not forecast or explain the variations in realized returns. The authors concluded that there might therefore be other, non-measured factors affecting the returns.

In order to see whether non-economic factors could affect returns, Cutler, Poterba and Summers examined the impact of national and international events on stock market returns. They collected a list of 49 important events in the period 1941-1987, and studied the stock market's reaction to these events. For example, the Pearl Harbor bombing resulted in a $4.37 \%$ drop in the S\&P index, while news of Nixon's election resulted in a $0.16 \%$ rise in the market. On average, the important events yielded a return of $1.46 \%$ compared to a normal average daily return of $0.56 \%$, and were 6.40 times as volatile. A complementary analysis examined the 50 largest price changes and news events by contrasting the market returns with stories in the New York Times. Cutler, Poterba and Summers found that the explanations for some price movements were "inadequate". The conclusion from the two analysis is that major news events are relatively rare and that they cannot explain the variation in day-to-day prices that stocks experience. The authors proposed that price movement was caused because investors did not trade "on their own assessment of values", but instead relied on others for opinions. In such a market, the authors explained, small changes in the leading investor's opinions could have great impact on stock market prices without any major events occurring.

Breen, Glosten and Jagannathan [Breen et al., 1989] proposed a model relating the excess return on stocks to the risk free rate. Using monthly data for the value and equally weighted portfolios on the NYSE, for 1954-1986, they examined the model's predictions with a switching portfolio consisting of holding stocks when the predicted excess return was positive, and holding bonds when the predicted returns were negative. The trading strategies were not very successful. For value weighted returns the strategy yielded $0.55 \%$ vs. $0.53 \%$ for the value weighted portfolio itself, and $0.64 \%$ vs. $0.79 \%$ for the equally weighted portfolio. The only redeeming quality of the trading strategy was the fact that its volatility was smaller than that of the raw value and equally weighted portfolios. The 
standard deviations of returns were 3.24 compared to 4.16 and 3.91 compared to 5.08 for the value and equally weighted portfolios respectively. The fit of the model was weak, with $R^{2}$ values of 0.02 for both types of returns. Regressing squared residuals on the interest rate variable, Breen et al. examined the relation between stock volatility and interest rates. They found that on average, volatility was higher when interest rates were high.

Breen et al examined the characteristics of the forecasts generated from the model by examining the forecasted returns during actual up and down markets. They tested the predictive ability using the regression $R_{t}=a_{0}+a_{1} I_{t-1}+v_{t}$, where $I_{t-1}=1$ if the model forecasted an up market at time $t-1$, and 0 otherwise, by examining the significance of the $a_{1}$ coefficient. They found $a_{1}=0.87$ for the value weighted and 0.46 for the equally weighted portfolios indicating that the model had forecasting ability and was able to identify periods of high and low returns. The coefficients were, however, found not to be significant, probably because of heteroscedasticity. Analyzing residuals from this regression, the authors also found that up market forecasts were characterized by lower variance than down market forecasts.

The trading strategy based on the value weighted portfolio yielded an excess return of $2 \%$ per year and the returns on the equal weighted portfolio were not significant.

\subsection{Time Series Models}

Univariate prediction models use past price information in order to predict future price behaviour - and are, in other words, extrapolative models. The goal of these models is to explain and predict the behaviour of stock returns using information about their auto-correlation structure. Two types of studies can be clearly distinguished in the literature: studies of short-term predictability of stock returns and studies of long-term predictability. As has been pointed out by Fama [Fama, 1991], short term returns show positive auto-correlations but long-term returns show negative auto-correlations: this is the puzzle the univariate models of returns are trying to explain.

One type of extrapolative model is the price-trend model which assumes that prices do not fully and instantaneously aggregate information, but instead respond to it slowly and incorrectly creating predictable trends in stock prices [Taylor, 1980].

The price trend model advocated by Taylor [Taylor, 1980] assumes that prices do not respond immediately to new information, but instead, do so progressively. 
Taylor's model expresses returns, $r_{t}$, as a function of a trend, $\mu_{t}$, representing the information progressively incorporated into prices, and an error term, $e_{t}$, as information which is understood clearly by the market and incorporated immediately. Formally:

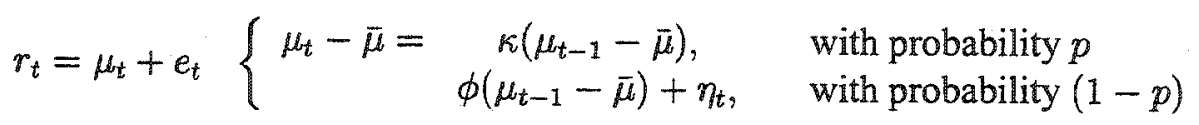

where $\bar{\mu}=\mathbb{E}(\mu), \eta_{t}$ is an error term, $\kappa, \phi$ are parameters and and $1-p$ is the proportion of trading days where new information appears. Different model configurations are possible by adjusting the parameters $\kappa$ and $\phi$ : the basic trend model corresponds to $\kappa=1, \phi=0$; the damped trend model to $\kappa<1, \phi=0$; the autoregressive trend model to $\kappa=1, \phi \neq 0$, and a continuous trend model results when $\kappa=0$ and $p=0$. These models can be generalized even further by the general trend model: $\mu_{t}-\bar{\mu}=\alpha_{t}\left(\mu_{t-1}-\bar{\mu}\right)+\eta_{t}$, where $\alpha_{t}$ is a random variable.

The autocorrelation function of the price trend model is: $\operatorname{Cov}\left(r_{t}, r_{t+i}\right)=$ $\operatorname{Cov}\left(\mu_{t}, \mu_{t+i}\right) / \operatorname{Var}\left(\mu_{t}\right)+\operatorname{Var}\left(e_{t}\right)$, and the autocovariance of the general trend model is $\operatorname{Cov}\left(\mu_{t}, \mu_{t+i}\right)=\mathbb{E}^{i}\left(\alpha_{t}\right) \operatorname{var}\left(\mu_{t}\right)$. Setting $p=\mathbb{E}\left(\alpha_{t}\right)$ yields a unique autocorrelation function for all the possible variations of the general trend model:

$$
\rho_{i}=p^{i} \frac{\operatorname{Var}\left(\mu_{t}\right)}{\operatorname{Var}\left(\mu_{t}\right) \operatorname{Var}\left(e_{t}\right)}=A p^{i}, \quad \mathrm{~A}>0 .
$$

The author uses this autocorrelation function to forecast returns for industrial and agricultural commodities as well as the dollar/pound exchange rate. The general model was estimated by finding values for $A$ and $p$ which minimized the difference between the theoretical and data autocorrelations: $S(A, p)=n \sum_{i=k_{1}}^{k_{2}}\left(A p^{i}-\right.$ $\left.\hat{\rho}_{i}\right)^{2}$, where $k_{1}, k_{2}$ represent the autocorrelations range.

A measure of improvement in the prediction MSE for the model over predictions from a random walk was developed based on the ability to forecast the future trend in prices $\mu_{t}$. The theoretical maximum improvement ranged from $0.1 \%$ to $0.2 \%$ for tin and coffee respectively.

Predictions were performed for $1,5,10$ and 20 days in the future, in-sample and out-of-sample. In-sample results show that the maximum theoretical reduction in MSE was tracked very closely for 1 day ahead forecasts with actual forecasts performing sometimes better than the theoretical limit. As the forecasting horizon lengthened, the theoretical reductions increased to $0.6 \%$ to $11 \%$, and the model reductions followed; additionally, as the forecast horizon increased, the model tended to slightly outperform the theoretical limits. Out of sample forecasts, performed only on copper, lead tin, zinc and sugar, performed acceptably: 
only the models for lead and tin were able to consistently decrease the MSE over the theoretical limit across the forecast horizons.

Conrad and Kaul [Conrad and Kaul, 1988] examined the relation between expected and real returns by modeling the relation with a state space model representing expected retums as a first order autoregressive process:

$$
\begin{aligned}
R_{t} & =\mathbb{E}_{t-1}\left(R_{t}\right)+\epsilon_{t} \\
\mathbb{E}_{t-1}\left(R_{t}\right) & =\phi \mathbb{E}_{t-1}\left(R_{t-1}\right)+u_{t-1}
\end{aligned}
$$

The behaviour of expected returns was examined for ten size portfolios using weekly data from July 1962 to December 1985. Model parameters were estimated separately for each size portfolio over the whole data period and the Kalman filter was used to extract expected returns. A separate model with a January dummy variable was also estimated.

The results showed that the estimated model parameter $\phi$ decreased with portfolio size, ranging from 0.59 for small stocks to 0.087 for the largest portfolio decile, and the January dummy variable was shown to be significant for all but the largest two portfolios.

Two models were then estimated to establish the ability of expected returns to explain the variation in real returns. The general form of the model was: $R_{t}=$ $\alpha+\beta_{1} \mathbb{E}_{t-1}\left(R_{t}\right)+\beta_{2} R F_{t-1}+\beta_{3} E W M R_{t-1}$, where $\mathbb{E}_{t-1}\left(R_{t}\right)$ is the expected return for period $t$ at time $t-1, R F_{t-1}$ the risk free rate at $t-1$ and $E W M R_{t-1}$ the market rate of return on the equally weighted market portfolio at time $t-1$. The first model constrained $\beta_{2}=\beta_{3}=0$.

Table 3.4 provides some parameter estimates for the models. The results from the first model show the expected returns to be unbiased estimators, but also to be poor explanators of real returns. Indeed the slope and intercept parameters for Model 1 are close to 0 and 1 respectively, indicating that the expected returns $\mathbb{E}_{t-1}\left(R_{t}\right)$ are fair estimates of the real returns, and this for all 10 portfolios. The explanatory picture is, however, grim: the extracted expected return can only explain up to $26 \%$ of the variance of the real returns, but only for the smallest portfolio of stocks, and the explanatory power of this variable falls with firm size. For larger portfolios $\mathbb{E}_{t-1}\left(R_{t}\right)$ can only explain less than $10 \%$ of the variance of real returns, and less than $1 \%$ for the largest portfolio of stocks.

The multivariate regression results provide further insight into the behaviour of real returns. It seems that except for the extracted expected returns, the additional variables in the model, interest and market returns rates, contribute little to the explanation of the variation in real returns. The interest rate variable is negatively related to the real return for all portfolios, as was to be expected, but is 


\begin{tabular}{llcccc}
\hline Statistic: & $\alpha$ & $\beta_{1}$ & $\beta_{2}$ & $\beta_{3}$ & $R^{2}$ \\
Model 1 - Pf. 1 (small) & -0.000003 & 1.0 & $\cdot$ & $\cdot$ & 0.265 \\
Pf. 5 & 0.000005 & 0.995 & $\cdot$ & $\cdot$ & 0.101 \\
Pf. 10 (large) & 0.0 & 1.0 & $\cdot$ & $\cdot$ & 0.007 \\
Statistic: & $\alpha$ & $\beta_{1}$ & $\beta_{2}$ & $\beta_{3}$ & $R^{2}$ \\
Model 2 - Pf. 1 (small) & 0.0012 & 0.849 & -0.67 & 0.17 & 0.273 \\
Pf. 5 & 0.0019 & 0.883 & -1.551 & 0.04 & 0.101 \\
Pf. 10 (large) & 0.0018 & 0.947 & -1.583 & 0.002 & 0.007 \\
\hline
\end{tabular}

Table 3.4: Conrad and Kaul estimates for the model: $R_{t}=\alpha+\beta_{1} \mathbb{E}_{i-1}\left(R_{t}\right)+$ $\beta_{2} R F_{t-1}+\beta_{3} E W M R_{t-1}$

nowhere significant. The same can be said of the market return variable which is weakly significant for portfolios 1 to 3 . The weak explanatory power of these variables is confirmed by negligible increases in the $R^{2}$ statistics for all models. The extracted expected returns therefore "subsume" information contained in the two explanatory variables.

Conrad and Kaul's study presents several deficiencies. First, model parameters have been estimated over the whole set of data, providing an illusion of "good fit" and inflated $R^{2}$ values. Had the models been estimated on a subset of the data, the explanatory results would probably have been much worse. Second, Conrad and Kaul re-estimated their models on five sub-periods of the data, and found that the explanatory power of expected returns changes dramatically over time (they do not provide the results of their analysis however). This indicates that most likely model parameters change through time and that the first order autoregressive model for expected returns may be invalid. These avenues of research are not explored however.

Jegadeesh [Jegadeesh, 1990] proposed to show that the autocorrelations detected in the different studies of market behaviour were economically significant. Assuming that returns for stock $i$ at time $t, R_{i, t}=\mathbb{E}\left(R_{i}\right)+\eta_{i, t}$, could be decomposed into expected $\mathbb{E}\left(R_{i}\right)$ and unexpected $\eta_{i, t}$ components, he proposed the following cross-sectional model of stock returns: $R_{i, t}=\alpha_{0, t}+\sum_{j=1}^{J} \alpha_{j, t} R_{i, t-j}+\epsilon_{i t}$.

In order to test the model's performance, Jegadeesh examined it's fit with respect to all stocks on the CRSP tapes and size quintiles for the period 1929-1982. 
The proposed form of the cross-sectional regression was:

$$
R_{i, t}-\bar{R}_{i, t}=\alpha_{0, t}+\sum_{j=1}^{12} \alpha_{j, t} R_{i, t-j}+\alpha_{13, t} R_{i, t-24}+\alpha_{14, t} R_{i, t-36}+\epsilon_{i t}
$$

where $\bar{R}_{i, t}$ is the average monthly return for security $i$ in the period $t+1$ to $t+60$, $R_{i, t-j}$ the return on security $i$ in month $t-j$ and $\alpha_{i, t}$ are model parameters.

The model estimated for all stocks found that coefficients $\alpha_{1}=-0.0923$ and $\alpha_{12}=0.0339$ were the most significant with t-values of -18.58 and 9.09 respectively, and the coefficients $\alpha_{13}$ and $\alpha_{14}$ were also found to be significant. A further test consisted of estimating the model for the months of January and February-December separately. January regressions showed that all coefficients apart $\alpha_{1}, \alpha_{12}, \alpha_{13}, \alpha_{14}$ were negative, while the February-December regressions showed that only coefficients $\alpha_{0}, \alpha_{1}$ were negative. Non-January regressions also found that the most significant coefficients also were $\alpha_{1}=-0.0801$ and $\alpha_{12}=$ 0.0297 with t-values -17.20 and 7.96 respectively. The model was not able, however, to explain the observed returns satisfactorily with an $R^{2}=0.11$ for all months data, and $R^{2}=0.18$ for January. Models estimated on size quintiles did not differ markedly from all stock returns, with the exception that January model coefficients were slightly higher for smaller stocks.

Jegadeesh next performed a predictive out-of-sample test consisting of three trading strategies with parameters estimated on the first five years of data. Strategy So consisted of ranking predicted returns in decreasing order into 10 portfolios, with the first portfolio consisting of the highest return stocks; strategy S1 consisted of ranking stocks in ascending order into 10 portfolios according to lag- 1 return and strategy S2 consisted of ranking stocks in descending order based on lag-12 return. The abnormal returns from the three strategies were then estimated using the market model.

Out of sample results indicated that for strategy S0, portfolio 1 averaged an excess return of $1.1 \%$, and portfolio $10,-1.38 \%$ per month, for a difference of $2.48 \%$ per month or $34 \%$ per year. Additionally the first five portfolios yielded positive excess returns, with the remaining portfolios yielding negative returns, with January retums being higher in magnitude compared to other months.

Strategies S1 and S2 yielded less impressive results with return differences between the best and worst portfolios of $1.99 \%$ and $0.93 \%$ respectively. The results also showed that the best portfolio for strategy S0 earned a positive return in $71 \%$ of all months while the worst portfolio earned a positive excess return only $20 \%$ of time. 
Jegadeesh next evaluated the three strategies in the light of transaction costs. Assuming a transaction cost of $0.5 \%$, Jegadeesh computed that the strategies SO and S1 would still be profitable, earning returns of $21 \%$ and $14 \%$ per year respectively. Strategy $\mathbf{2} 2$ would, however, yield economically insignificant results. It is interesting that strategy SO showed profitability in the light of a high stock turnover - on average, $91 \%$ of stocks in the difference portfolio changing every month, and thus contradicting earlier results.

Zhou [Zhou, 1996] examined the predictive performance of the Conrad and Kaul [Conrad and Kaul, 1988] and Fama and French [Fama and French, 1988b] models of stock returns. Zhou's emphasis in the study was to test the models' ability to forecast both short and long-term returns, something the models were not designed to do. The first model expressed returns as an autoregressive process, and was designed for forecasting short-term returns, while the second one, allowed for a permanent and transitory components of stock prices. The two models are as follows:

$$
\begin{array}{r}
r_{t}=\mu_{t-1}+\epsilon_{t} \\
\mu_{t}=\phi \mu_{t-1}+\eta_{t} \\
r_{t}=\Delta z_{t}+\epsilon_{t} \\
z_{t}=\phi z_{t-1}+\eta_{t},
\end{array}
$$

where $r_{t}$ is the return at time $t$ and, $\mu$ and $z$ are state variables. The forecasts were performed using the Kalman filter.

Regressing realized returns on their forecasts, Zhou found that the first model was a good predictor for one month ahead data, however, as the predictive horizon extended (up to 10 years), the forecasts became more and more biased. The theoretical correlation of the model was shown not to match the empirical correlation of the data, explaining the lack of predictive power at longer horizons. Additionally, the dividend yield variable has been shown to have explanatory content over the expected return.

The second model, used first in Fama and French [Fama and French, 1988b] was designed to explain long term stock return movements. As expected, regressing realized returns on expected returns showed that the model's performance for long returns exceeded that of the first model, but the first model was still the better performer on short term returns. A similar picture was told by the autocorrelation structure: the model matched the empirical correlation of long-horizon returns better, than that of the short-term returns. 
In order to improve the explanatory power, Zhou created a third model designed to account for short-term and long-term characteristics of returns. The third model had the following form:

$$
\begin{array}{r}
r_{t}=z_{t}-z_{t-1}+\epsilon_{t} \\
z_{t}=\phi z_{t-1}+\gamma x_{t-1}+\eta_{t} \\
x_{t}=\lambda x_{t-1}+\xi_{t}
\end{array}
$$

where $z$ characterized the transitory effect, and $x$ characterized short stock movements. The model was found to (for short and long horizon returns) perform slightly better than either of the two previous models. The model was also able to fit the empirical autocorrelations better than the previous models, being able to fit both short and long term autocorrelations. Regressing realized returns on expected returns and dividend yields showed the dividend yield variable to be insignificant, confirming the explanatory power of the new model.

While this study seems to be encouraging, there are several areas of concern regarding the modelling and the methodology. First, the reason for the given formulation of the third model is not provided, and the model is not compared with possible alternatives. Secondly, the models used in the study were estimated on the full sample of data, diminishing the value of the good predictive performance. Thirdly, the performance was not analyzed in terms of error measures, but rather in terms of unbiasedness and correlation fit. While such a comparison was interesting and valuable, the forecasting performance would have been better measured through the MSE and profitability criteria.

\subsection{Technical Trading Rules}

Technical analysis is the study of the historical stock market prices in the belief that they contain information as to the prices' future movements. Technical analysts believe that as different market participants analyze diverse sources of data, their findings will ultimately be reflected in prices, although not immediately, but progressively, as more and more market participants become aware of the new information. By studying past prices, and the trends within them, technical analysts claim to be able to forecast future movements of stock prices (Alexander [Alexander, 1961]). Neftci [Neftci, 1991] describes technical analysis as "a broad class of prediction rules with unknown statistical properties, developed by practitioners without reference to any formalism". 
But why use trading rules and not statistics? Trading rules are a response to the inability of theory and statistics to specify models of returns that adequately describe the behaviour of retums. In particular, trading rules are a response to the difficulty of specifying nonlinear models for the behaviour of retums. While statistical tools can easily specify linear relationships between random variables, there are no explicit tools to capture nonlinear behaviour (at least in the general literature). This weakness of statistics has lead market practitioners to consider new techniques of predicting behaviour, even at the expense of formality. The two worlds are not, however, totally isolated from each other. There seem to exist relations between statistical and trading rule methods whose combination can lead to surprising results (see Neftci [Neftci, 1991]).

The first empirical studies of technical analysis were performed by Alexander [Alexander, 1961, Alexander, 1964]. In his first paper, Alexander revisited the studies by Kendall [Kendall, 1953], Osborne [Osborne, 1959] and Cowles [Cowles, 1960] and re-examined the question of independence of stock returns.

Alexander first hypothesized that the near zero correlations from Kendall's study were the result of an interaction between long term and short term market trends. He proposed that the market was composed of an underlying long term trend on which are super-posed temporary swings such as "profit taking", which are negatively correlated with the underlying trend. He hypothesized that shortterm autocorrelations should be negative, those of medium-term returns should be zero because of the cancellation of the two price components, and longer horizon correlations should be positive evidencing the underlying trend.

By recomputing long term correlations from Kendall's results, he found that one and two weeks' returns were positively correlated, and as the retum interval lengthened, the correlations became negative, refuting his hypothesis. The correlations for the overall industrial average were as follows: 1 week, $0.234 ; 2$ weeks, $0.207,4$ weeks, 0.018 and 8 weeks, -0.063 .

Alexander then compared the variance of the indice's returns to that of a 21 lag moving average fitted to the returns data. Had the fit been perfect, the ratio of variances would be equal to 1 , however, the results showed that the ratio of variances hovered around 0.2 , with only the Investment Trusts index scoring a ratio of 0.525 . This indicated clearly the absence of major underlying trends in the data.

Alexander then examined Osborne's proposition that the changes in log-returns are normally distributed. The $\chi^{2}$ test ( $X^{2}=60$ with 8 d.f.) found that the normal distribution does not fit the data well, particularly in the tails. This finding however did not give any insight as to the nature of the dependence in returns. 


\begin{tabular}{lcccccc}
\hline Index & \multicolumn{4}{c}{ Filter Size } & Buy-and-Hold \\
& $5 \%$ & $10 \%$ & $15 \%$ & $25 \%$ & $50 \%$ & \\
\hline DJIA (1897-1914) & 20.5 & 4.6 & 6.6 & 2.6 & -3.9 & 3.2 \\
DJIA (1914-1929) & 15.8 & 10 & 9.9 & 11.1 & -2.1 & 14.1 \\
SP500(1929-1959) & 36.8 & 16.8 & 6.9 & 8.2 & 8.5 & 3 \\
\hline \hline
\end{tabular}

Table 3.5: Alexander's [Alexander, 1961] Filter Rule Profits

Alexander further tested the dependence of returns by looking at the distribution of runs in the returns, but unlike Cowles, he found that the pattern of runs on the DIIA resembles that generated by a random walk.

Alexander, recognizing that the evidence strongly supports the random walk hypothesis, decided to perform a final test making the assumption that underlying trends in the market were "masked by the jiggling of the market". In order to identify the underlying long term trend in the market he proposed a trading rule for eliminating the noise in price movements.

The "filter rule" consisted of buying a stock after an $x \%$ appreciation relative to a low and holding it until it declined $x \%$ from a following high and then going short, while ignoring all price movements of less than $x \%$. Alexander examined the profitability of the rules by testing filters from $5 \%$ to $50 \%$ on the monthly DIIA (1897-1929) and SP500 (1929-1959) indices. Under the null hypothesis of a random walk no filter was to be profitable, the buy-and-hold strategy being the best performer. The results showed, however, that profits were possible. The small filters were the most profitable with profitability diminishing with increases in filter size. The filter results are presented in Table 3.5.

These results clearly indicate that stocks do not follow a random walk since trading rules that outperformed the buy-and-hold strategy were established. The results, however, did not incorporate the effects of transaction costs which could be substantial for the small filters due to the large number of trades they trigger. Alexander did not provide any detailed analysis of costs in this study to confirm this opinion. He concluded by arguing that while the returns seemed to follow a random walk through time, they were dependent on the size of "moves". In other words, Alexander argued that "if the stock market has moved up $x \%$ it is likely to move up more than $x \%$ further before it moves down by $x \% "$.

Fama and Blume [Fama and Blume, 1966] reexamined Alexander's trading rules by studying their performance on individual stocks instead of stock indices. Using daily data for the 30 stocks composing the Dow Jones average from Jan- 
uary 1956 to September 1962 they simulated 24 filters from $0.5 \%$ to $50 \%$. Unlike in Alexander's study, Fama and Blume have taken into account trading costs and dividend effects in examining the performance of the trading rules.

Fama and Blume's buy-and-hold strategy yielded a retum of $6.2 \%$ for the period considered while the dividend adjusted strategy yielded $9.8 \%$. The filter profits did not fare as well. Ignoring dividends, the average profit from filters was $1.05 \%$, and, with dividend adjustment, the returns decreased to $0.32 \%$. The problem was caused by short positions which on average yielded a loss of $12.79 \%$ compared with an average gain of $8.22 \%$ on long positions. This average gain of $8.22 \%$ on long positions was realized thanks to the fact that for 13 out of the 30 stocks in the DJIA the filter rule realized average returns greater than for the buy-and-hold strategy.

Next Fama and Blume analyzed the profits for the individual filters. The filter performance was very bad compared to the buy-and-hold strategy. Before commissions only 8 filters were showing negative returns. After commissions all but 6 filters yielded negative results. The only filters with positive returns were of sizes $12 \%$ to $25 \%$ with an average return of $2.1 \%$ compared to $9.8 \%$ for the buy-andhold strategy. The small filters, ignoring commissions yielded the most profits, however, considering the fact that the $0.5 \%$ filter generated 12514 transactions for the period under study, and given an average transaction cost of $0.1 \%$, the profits were all but eliminated. Although large filters did not suffer from excessive transaction costs, they did not yield satisfactory returns.

Sweeney [Sweeney, 1988] revisited Fama and Blume's study by reexamining the performance of their filter rules on the "most promising" stocks from the 1966 study. They examined 14 stocks which beat the buy-and-hold strategy in Fama and Blume and tested them on the $0.5 \%$ filter on daily data in the period 1970-1982. Unlike Fama and Blume, Sweeney examined the performance of the filter rules while assuming differential transaction costs for different types of market participants such as traders, institutional investors and private individuals. He found that for a transaction cost of $0.05 \%$, the cost to floor traders, the $0.5 \%$ filter rule outperformed buy-and-hold for all of the 14 stocks, and that with costs of $0.1 \% 11$ out of 14 stocks were still profitable. The combined stock performance was $14 \%$ for costs of $0.05 \%$ and of $10 \%$ for costs of $0.1 \%$ per transaction. Money managers could expect $2 \%$ gains with transaction costs of $0.2 \%$. Private individuals, on the other hand, were not likely to profit from a filter strategy.

Brock, Lakonishok and LeBaron [Brock et al., 1992] tested and attempted to explain the performance of two of the most popular trading rules: the moving average oscillator and trading range break. The moving average trading rule is 
characterized by a short-term (ST) and a long-term (LT) moving averages. A buy signal occurs when the short-term moving average rises above the long-term moving average, and a sell signal occurs in the opposite case. The trading range break generates buy signals when the price rises above a local maximum price, and sell signals when the price falls below the local minimum price. Both of these trading rules are based on intuition. The moving average rule in effect performs a smoothing operation on the price series allowing one to detect trends. The trading range break strategy assumes the existence of local trends. When a price rises above a local maximum, the price is likely to continue rising, and conversely for price declines.

The authors implemented three trading strategies: Variable Moving Average (VMA), Fixed Moving Average (FMA), and, trading range break (TRB) strategies. In total 5 moving average trading rules were implemented: 1-50, 1-150, 5 $150,1-200$ and 2-200 (1-50 means ST moving average of 1 day, and a LT moving average of 50 days). All five moving averages were augmented with a "whiplash" band of $0 \%$ and $1 \%$ for a total of 10 trading rules. The first strategy, VMA, generated buy signals when the ST average was above the LT average plus the band range, and a sell signal in the converse case. When the ST average was inside the band, no signals were generated. The second strategy, FMA, computed returns exclusively for a period of 10 days after the ST average moved above the LT average. Finally, the TRB strategy computed the required maxima over periods of 50,150 and 200 days, and returns exclusively for 10 days after a signal has been recorded. A "whiplash" band was also in effect for the TRB yielding 6 strategies overall.

The study used daily data from the DJIA index from 1897 to 1986 and 4 sub-periods. One and ten day non-overlapping returns were computed from logdifferences of prices of the index. The average returns are positive for the whole period because of the up-trending market - the average one day return is 0.00017 and the ten day return 0.0017 . The returns are characterized by high kurtosis and skewness (see Table 3.6, page 59). The variance of returns was highest for the Great Depression period (1/1/1915-31/12/1938). The first order autocorrelation is significant for the two return types. However, for the one day returns for all subperiods the first order autocorrelation is positive while the second order correlation is negative.

The results from the technical analysis tend to reject the random walk hypothesis - all the trading rules are profitable, and the average return from trading rules is greater than the DJIA average return. Table 3.7 , page 60 , presents a summary of the results consisting of the best and worst performing trading rules for 


\begin{tabular}{l|c|c}
\hline Statistic & 1 day returns & 10 day returns \\
\hline Mean & 0.00017 & 0.0017 \\
St.Dev. & 0.0108 & 0.0351 \\
Skewness & -0.1047 & -0.4583 \\
Kurtosis & 16 & 7.91 \\
$\rho(1)$ & 0.033 & 0.037 \\
$\rho(2)$ & -0.026 & 0.018 \\
\hline \hline
\end{tabular}

Table 3.6: Summary of DJIA average for 1897-1986

the three categories, together with the average results for each trading rule type. The caption "w/band" indicates the results for the trading rules executed with the $1 \%$ "whiplash" band.

Several interesting findings can be noted. First, the average return after a "buy" signal has been issued by a trading rule is always positive, and conversely for "sell" signals. Second, the differences between the returns from the buy and sell signals are all positive - the test that the difference "Buy-Sell" is 0 is rejected for all trading rules. Additionally, the tests that the "Buy-Sell" returns are equal to the unconditional returns on the DJIA are rejected either strongly or marginally. Thirdly, the addition of the safety, or "whiplash" band, around the trading rules tends to improve their performance. As Table 3.7 shows, the worst performing trading rules are those without the safety band; the the best performing rules always include it. Brock, Lakonishok and LeBaron argue that the strong performance of the trading rules compared to other studies is caused by the large amount of data giving them more power to reject the null of random walk.

To better examine the trading rules, the authors fitted 4 models to the returns data: they estimated a random walk, AR(1) model, a GARCH in mean model and an EGARCH model. Next they have simulated the estimated models, performed a trading rule analysis on the artificial series and compared with the results obtained from the real DJIA data. Table 3.8 summarizes the results of the simulation and presents the values of the "Buy-Sell" differences in returns for the various trading rules and simulated models.

The results from the random walk simulation yield several surprises and are summarized in the first row of Table 3.8. First, the trading rules did not perform well on the random walk and were not able to generate any profits. The returns following buy signals were always greater for the DIIA data than for the simulated data, and conversely for sell signals. Secondly the variance of returns following 


\begin{tabular}{l|c|c|c}
\hline Trading Rule & Buy signal return & Sell signal return & Buy-Sell \\
\hline Best VMA $(1,50,1)$ & 0.00062 & -0.00032 & 0.00094 \\
Worst VMA $(5,150,0)$ & 0.00037 & -0.00017 & 0.00053 \\
Average w/band & 0.00040 & -0.00023 & 0.00062 \\
Average w/o band & 0.00044 & -0.00027 & 0.00071 \\
Average VMA rule & 0.00042 & -0.00025 & 0.00067 \\
\hline Best FMA $(1,200,1)$ & 0.0058 & -0.0077 & 0.0135 \\
Worst FMA $(1,200,0)$ & 0.0050 & -0.0019 & 0.0069 \\
Average w/o band & 0.0054 & -0.0023 & 0.0077 \\
Average w/band & 0.0052 & -0.0057 & 0.011 \\
Average FMA rule & 0.0053 & -0.0040 & 0.0093 \\
\hline Best TRB $(1,150,1)$ & 0.0086 & -0.0035 & 0.0120 \\
Worst TRB $(1,50,0)$ & 0.0050 & 0 & 0.0050 \\
Average w/o band & 0.0046 & -0.0017 & 0.0064 \\
Average w/band & 0.008 & -0.003 & 0.0109 \\
Average TRB rule & 0.0063 & -0.0024 & 0.0087 \\
\hline \hline
\end{tabular}

Table 3.7: Trading rule results from Brock, Lakonishok and LeBaron [Brock et al., 1992]

\begin{tabular}{l|c|c|c}
\hline Model & VMA & FMA & TRB \\
\hline RW & 0 & 0.00016 & 0.00009 \\
AR(1) & 0.00006 & 0.00104 & 0.00118 \\
GARCH-M & 0.00018 & 0.00222 & 0.00410 \\
EGARCH & 0.00002 & 0.00151 & -0.00043 \\
DJIA Data & 0.00067 & 0.00930 & 0.00871 \\
\hline \hline
\end{tabular}

Table 3.8: Simulation results: average "Buy-Sell" returns for the VMA, FMA and TRB trading rules 
buy and sell signals does not correspond to the view that higher returns are earned when variance is higher. The trading rules seemed to issue buy signals when the markets were less volatile and sell signals when markets were more volatile.

The AR(1) model attempted to explain whether correlation in returns could be the cause of the trading rule profitability. The average "Buy-Sell" return for the AR(1) process with the VMA trading rule resulted in a return of $0.00006 \%$ compared to $0.00067 \%$ for the DIIA data - this is not a strong performance. The model also cannot explain the difference in variances for buy/sell signals.

The GARCH-M model cannot explain the trading rules either. The average returns for the GARCH-M model are presented in row 3 of Table 3.8: the GARCH$\mathrm{M}$ model is unable to explain fully the relative return, but more importantly yields positive results after sell signals. The model also generates very high volatility for buy periods, which is inconsistent with earlier findings.

The EGARCH model also explains the real returns badly. The total "BuySell" difference is nowhere close to the one observed in the DJIA series (row 4 Table 3.8), and is even negative for the TRB rule. It was, however, much better able to match the volatilities observed in the DJIA data than the GARCH-M model. With this smaller variance, however, it cannot explain the larger returns for the buy and sell signals.

The authors conclude that the process generating prices is complex, most likely nonlinear, and cannot be explained by either the random walk, AR(1), GARCH-M, or EGARCH models. Most importantly, the authors found that risk could not explain most of trading rule profits. Even though the trading rule profits were significant, the study did not account for trading costs or dividends and thus cannot ensure the question of actual performance.

Brown, Goetzmann and Kumar [Brown et al., 1998] have measured the predictive ability of W.P. Hamilton's Dow Theory based on his stock recommendations. Hamilton, while editor of the Wall Street Journal (from 1902-1929), used to regularly write editorials in which he would predict the future course of the market. While the editorials' predictive contents were tested before, with negative results, Brown, Goetzmann and Kumar [Brown et al., 1998] contributed to the literature by testing their performance with more modern tools.

Their analysis consisted of examining whether the behaviour of the market between editorials was consistent with the previous editorial's recommendation based on whether it outperformed the risk free rate. Examining Hamilton's 255 editorials, the authors classified the predictions as "Bullish", "Bearish" and "Neutral", finding that the editorials are Bullish $54 \%$, Bearish $24 \%$ and Neutral $22 \%$ of the time. Additionally, they found that Hamilton was able to predict (not counting 
Neutral predictions) an up market 74 out of 130 times and a down market 36 out of 54 times, an ability found to be significant at the $1 \%$ level. The Hamilton-Merton test of timing ability further confirmed that Hamilton was able to forecast market falls and rises.

Brown, Goetzmann and Kumar next tested the performance of Hamilton's recommendations over time. The return on a portfolio following Hamilton's recommendations yielded $10.73 \%$ with a Sharpe ratio of 0.559 compared to $10.75 \%$ for the Cowles' market index which had a Sharpe ratio of 0.456. The Sharpe ratios said clearly that Hamilton's strategy was better at exploiting risk, which was confirmed by a Jensen's alpha of $4.04 \%$.

The authors next tested the random walk hypothesis by simulating the course of the stock market using the bootstrap to generate random samples from the distribution of stock returns for the period 1902-1929 and applying Hamilton's recommendations to the artificial series. Comparing the profits on the random sample with those obtained from the real DIIA data, the authors found that applying Hamilton's recommendations on the artificial data yielded an average total return of $5.32 \%$. The Sharpe ratio and Jensen alpha for the real data were also higher than for the simulated series, indicating that a random walk does not capture all the behaviour of stock returns.

In order to test Hamilton's recommendations' predictive content, the authors generated random recommendations and applied them to the real data. This test had the advantage of not destroying the dependence structure of returns. The authors found that Hamilton's trading rules had a predictive ability for stock returns, since the random strategies resulted on average in a $5.12 \%$ return. This lead the authors to conclude that Hamilton did not generate his recommendations by flipping a coin.

Subsequently the authors conducted an "event study" type test to determine whether the market went up after buy, and down after sell recommendations. The authors examined the behaviour of returns 40 days before and after a "signal" and found that buy signals were usually followed by a $1.5 \%$ increase and sell signals by a $1.74 \%$ decrease in the market. Interestingly, the buy and sell signals were on average by positive and negative trends respectively.

Brown, Goetzmann and Kumar's most interesting contribution was an attempt at the identification of Hamilton's trading rules. Since Hamilton has not disclosed the method behind his recommendations, but has only presented his recommendations in his editorials, the authors have attempted to extract rules by studying the pattern of buy and sell signals that he produced. Their first attempt focused on regressing Hamilton's "bear" calls on various variables related to past return 
performance. They found that the most significant variables were related to the interaction between 60-day returns and 30-day returns. This suggested that the DOW strategy was based on momentum, with buy and sell signals depending on the trend in the movement of prices in the past 60 days.

The authors then proceeded on a Frankenstein-like quest of recreating Hamilton in a neural network (or at least his analytical skills). Their approach consisted of mapping technical analysis elements into Hamilton's recommendations in order to extract rules useful for predicting the behaviour of stock prices. (As a note, the use of NN actually did not allow the authors to extract the exact rules used by Hamilton, in the sense that the rules that were found, were still encoded in the estimated NN model, and thus were not easily interpretable, or not interpretable at all, by the researchers. Therefore, while the NN might have identified some rules, it did not make them explicit.) Once trained, the authors then used the "model" to predict the behaviour of daily DJIA returns over the period September 1930-December 1997. The results showed that the NN model tended to outperform the buy-and-hold strategy for most of the testing period. The buy-and-hold yielded $7 \%$ and the artificial-Hamilton strategy close to $10 \%$. Sub-period results, on the other hand, were varied. The NN strategy outperformed the buy-and-hold in some periods like the 1930 s ( $1.5 \%$ for buy-and-hold and $11.1 \%$ for $\mathrm{NN}$ ) and underperformed in the $1980 \mathrm{~s}$ ( $12.6 \%$ for buy-and-hold and $11.3 \%$ for NN). The lack of a trading cost analysis may have inflated the profitability of the trading rules however.

In the end, the authors concluded that Hamilton was successful at forecasting market behaviour, a conclusion vindicated by the ability of his trading rules to forecast returns with relative success in the 1930-1997 period.

Allen and Karjalainen [Allen and Karjalainen, 1999] introduced a new approach to the study of trading rules consisting of extracting "rules" from stock returns data using genetic programming. Their principal goal was to "find out whether in a certain sense optimal rules can be used to forecast future returns", and in contrast to Brown, Goetzmann and Kumar, Allen and Karjalainen wanted not only to estimate a model, but to extract actual rules from the data. The methodology involved the use of genetic algorithms in the form of genetic programming to find the best possible combination of simple functions of past data to predict future returns. In other words, the genetic programming approach was used to construct decision trees whose nodes consisted of simple functions of a limited number of arguments. For example, the function average computed the past average of stock prices in a window of time selected by the genetic algorithm to be optimal with respect to a certain objective function. Other functions 
consisted of the maxima and minima of prices in windows of time, the arithmetic operations, boolean functions etc... (please refer to Allen and Karjalainen [Allen and Karjalainen, 1999] Section 3) The goal of the genetic algorithm was then to find an optimal decision tree, composed of the simple functions that will best predict future returns. In other words, this technique represents a pure automated search for a trading rule.

The objective function consisted of the spread between the results of the trading rule and that of the buy-and-hold strategy during the "training period". In order to make the results more realistic the trading rule performance was adjusted for transaction costs of $0.1 \%, 0.25 \%$ and $0.5 \%$ per transaction. To avoid overfitting the authors also proposed a cross-validation rule consisting of split-sample testing. In particular a trading rule was accepted only if if it outperformed the previous best trading rule on the cross-validation sample. The algorithm terminated if there were no further improvements in profitability on the cross-validation sample and the last trading rule was selected as the optimal solution.

The authors applied their model to daily SP500 data from January 1928 to December 1995 divided by it's 250 day moving average to compensate for the autocorrelation in the index. The algorithms were "trained" on 105 -year periods, and cross-validated on the subsequent 2 -years of data. The testing sample consisted of data from the end of the cross-validation period to the end of the data-set in 1995. Ten iterations of the genetic algorithm were run for each training period for a total of 100 iterations and yielded 89 final trading rules.

For the $0.25 \%$ trading cost scenario the results indicated that almost none of the trading rules were able to generate returns in excess of the buy-and-hold strategy during the testing period. The average excess return for all the trading rules was negative $-2.05 \%$. The rules were able to weakly discriminate between periods of low and high returns. Most of the "Buy-Sell" returns were significantly positive just as in Brock, Lakonishok and LeBaron [Brock et al., 1992]. The results also revealed that the trading rules were somehow able to distinguish between periods of high and low volatility. When the trading rules held the market index market volatility was lower than during the periods when the rules held bonds. This success in forecasting periods of low and high activity in the market resulted in a variance of retums on the trading rules $(10 \%)$ significantly different from that of the SP500 index (14\%). Trading rules using the other transaction cost scenarios performed in a similar fashion without being able to yield overall positive excess returns.

The authors next presented two examples of trading rules selected by the genetic algorithm: price*minimum (price) $-0.0688^{*}$ price $>0.8943$, and 
lag (price, 1) >average (price/(|price-1.1727|)). The first rule issued buy signals when the square price exceeds 0.89 , while the second rule would issue a buy signal when the lagged price exceeded a moving average of prices of length price / (|price-1.1727|).

Overall the results from the study are disappointing and paint a picture of an efficient market. The study is, however, not perfect and raises more questions than usual studies of trading rules. The most important question concerns trading rule selection. The trading rule selection process illustrated in the paper is a blind search for an "optimum" trading rule with the only restrictions being the number and nature of the basic functions. As such, it is very difficult to make sense of the process's output, interpretation being an almost impossible task. Another issue is the "quality of fit". Genetic algorithms are known to be very good optimizing tools thus raising questions about over-fitting. In the light of this fact it is very possible that the genetic algorithms, instead of fitting, have actually found a local solution for the training sample instead of a real trading rule. Even though the authors used a cross-validation sample it is very likely that the search procedure found a solution "conditional" on the cross-validation sample. One has to remember that cross-validation is not a magic solution to the problem of over-fitting, but that it is simply a statistical method designed to help alleviate the problem of over-fitting and is not to be used blindly. In the end it seems that the study is a fine example of a fancy technique applied without a careful analysis of it's implications, and whose conclusions should not be taken at face value.

Neftci [Neftci, 1991] attempted to describe technical trading rules with common statistical models. He considered technical analysis as "a broad class of prediction rules with unknown statistical properties, developed by practitioners without reference to any formalism". His article attempted to formalize technical trading rules in order to gain a better understanding of their behaviour and performance.

He identified three major types of trading rules: trend crossing methods, moving average methods, and pattern analysis. The first category of techniques issues buy signals when a local level is crossed, the second when two moving averages cross, and the third when a pattern of a particular shape occurs. Neftci's question was whether these three categories could be translated into mathematical terms and used in "proper" prediction models. To prove whether these three groups of trading rules could be formalized properly the author used the theory of Markov times. The idea behind Markov times is that any proper forecasting strategy should use past or current data in its forecasts and should not depend on future data. Neftci proved that only the moving average class of trading rules could be 
formalized properly into a prediction method.

Neftci then asked the intriguing question of under which circumstances can technical analysis be useful and proved that in order to be useful, the process under analysis, namely stock prices, must be nonlinear in nature. If the process were linear the optimal prediction method would be constituted by a linear model. The strength of technical analysis may thus lie in the detection of simple nonlinear trends in the data over and above simple linear models, but only if the process followed by prices is nonlinear.

Neftci performed an empirical test using monthly data for the DJIA from 1792 to 1976 . The author computed a moving average trading rule and created a dummy variable $D_{t}$ taking on the value 1 if the the DIIA crossed above its 150 day moving average, -1 in the contrary case, and 0 in the case when no crossings occurred. Neftci then incorporated this dummy variable into a vector autoregression: $X_{t+\mu}=\sum_{i=1}^{k} \alpha_{i} X_{t-i}+\sum_{i=1}^{k} \beta_{i} D_{t-i}+\epsilon_{t+\mu}$, where $X_{t}$ represented the stock price. The hypothesis stated that if stocks followed a linear process the dummy variables would be insignificant in the model. The author tested the 12 months ahead forecasting model for the sub-periods 1795-1851, 1852-1910 and 1911-1976. The dummy variables were found to be insignificant for the first two periods, but were highly significant for the last period, with positive signs.

The study suffered from the fact that no measures of sample performance apart $R^{2}$ were provided. While an $R^{2}$ of 0.91 for the $1911-1976$ period is impressive compared to other studies it is only an in-sample measure which says nothing of the out-of-sample capability of the method. Had the study performed an out-ofsample check, it would have provided much needed information to researchers.

The literature on trading rules agrees to disagree on the value of technical analysis. Earlier studies seem to support the EMH while the more recent research seems to confirm a certain level of market inefficiencies. It is important to note however, differences between these early and modern studies.

\subsection{Conclusion}

This chapter has presented the multitude of predictive models and approaches found in the literature. The literature being vast, it has not been possible to present all the findings or conclusions, but it is believed that the review gave an accurate picture of the "state of the art" in stock market forecasting.

Roll [Roll, 1988], dissatisfied with the inability of financial models to predict behaviour of financial prices, said the following, "The immaturity of our science 
is illustrated by the conspicuous lack of predictive content about some of its most intensely interesting phenomena, particularly changes in asset prices". Recent research (see section 2.5) has proven that there is a large amount of dependence in stock returns. Returns are correlated at short and long lags, and under and overreaction in retums offers a possibility for earning excess profits. The review of forecasting models (chapter 3) has shown, however, that commonly encountered models often lack out of sample predictive power. On the other hand, the trading rule literature indicates that simple technical rules can predict the behaviour of stock prices.

The most important advance of the recent years has been the inclusion of the human element in the study of financial markets. Behavioural finance has shed not only a new light on the behaviour of returns, but has also attempted to explain the various anomalies found in the financial markets by using concepts borrowed from psychology. Investors are no longer assumed to be rational automata, but rather imperfect decision makers whose personal biases or interaction affect security prices in yet to be understood manners. Thaler [Thaler, 1999] predicts that behavioral finance will blend in with the conventional approach to yield a new and better understanding of the financial markets.

In the meantime, Roll's quote illustrates quite well the state of affairs and of knowledge in the field of finance concerning researchers' ability to explain and predict stock prices and returns. Even though the behavioral finance literature has shown predictability to exist, the statistical models reviewed earlier have a rather poor explanatory power - the $R^{2}$ statistics are low. While the tests of predictive power are not uniform across the literature, few studies display the ability to beat the buy-and-hold strategy. It is believed that a statistical model combining economic insight together with a realistic framework of investor behaviour will yield better predictions than those which have been produced to date. 


\section{Chapter 4}

\section{A New Approach to Forecasting Stock Returns}

\subsection{Introduction}

Recent studies have established significant amounts of predictability in stock returns. In particular it is well recognized now that relatively simple univariate and multivariate linear models are able to predict to a certain extent short term movements in stock index returns and that commonly-known trading rules can yield abnormal excess returns. It is also widely accepted that the behavior of returns is complex and not completely understood. For example, it has been discovered that returns exhibit many interesting characteristics such as non-normality and heteroscedasticity; questions regarding the nonlinear dependence of returns still remain unanswered.

The literature review has shown a large discrepancy between what is known of the behavior of returns and the manner in which they are being modelled and forecasted. The review of the literature indicates that researchers have ignored the findings about the nature of returns and insist on using "standard" modelling methodologies in predicting returns, and, it is therefore rather surprising that these simple techniques have achieved the levels of predictability reported in the literature.

This chapter proposes a new approach to the prediction of daily stock returns with the hope of yielding new insights into the behavior of markets and investors. 


\subsection{A Model of the Investor}

This study proposes a new approach to forecasting stock returns based on the idea that in order to model and predict stock returns successfully it is necessary to reproduce the pricing process effectively followed by market participants in valuing stocks. In order to better predict stock returns, it is believed that an empirical model of returns must not simply relate external variables to firms' returns, but must emulate the process used by investors in determining stock prices. The literature does not seem to feature such a particular approach to forecasting returns, and therefore, it is important to examine this new modelling idea.

The investors' and speculators' goal is to make trading profits by investing into stocks which balance expected retums with their riskiness. In order to realize their goal, investors and speculators search the market for profitable investment opportunities by analyzing firms' future business prospects using various types of information related to the condition of firms, as well as the markets, and the economy in general. The goal of this process is the estimation of the firms' value, or state, as related to their future performance. The value of the firm - the realistic expectation of its future profitability - is established based on indicators of its performance such as financial statements, news releases, stock prices and other sources designed to help identify firms worthy of investment. Once estimated, the state, in turn, is used by investors in making investment decisions and thus establishing share prices.

Investors, in their analysis of investment opportunities, however, are faced with a significant problem related to the fact that the data they collect is never totally accurate or complete. For example, economic data and financial statements are only released at distinct intervals of time, and the information they contain, while representative of the state of the economy and the state of the firms at a given date may not be entirely accurate. The investors' job is therefore to assess the impact of the limited and inaccurate news and information on corporations' future earnings and to form the best possible picture of the corporations' future performance.

Unfortunately, since investors do not observe the day to day internal workings of corporations and so do not have perfect knowledge of the corporations' current condition (except for a limited number of insiders), they cannot know with certainty, and can only estimate the effects and meaning of new information on the future earnings prospects of the various firms.

More precisely, in order to "incorporate" news into stock prices, investors need to accomplish two things as follows. In order to "value" the impact of new 
information, the investor's first step consists of forming an a-priori estimate of the state, or condition, of the firm. The investor forms the a-priori assessment by observing and gathering information on the firm (in the form of past financial statements, past analyst reviews, etc...) and analyzing the firm's environment. However, since the data at his or her disposal is not perfect nor complete, and his or her analytical skills are not superhuman, the investor's a-priori estimate will only be an approximation of the future financial profitability of the firm the investor, just as Bachelier suggested in his seminal work, develops a range of most likely values for the state of the firm, or a probability distribution of the value (or state) of the firm.

Once new information arrives, the second step consists of incorporating the latest news' impact into a new, or updated estimate of the firm's future profitability. The investor accomplishes this by weighting the importance and accuracy of the new information with that of the firm's a-priori state already established, in fact refreshing his or her probability distribution of the firm's value.

The investor uses the news for two purposes: first to augment his or her knowledge of the firm, or, in other words, to gain better knowledge of the condition of the firm, and second to confirm or refute certain aspects of his or her previous estimate, in effect improving the accuracy of his or her estimate. If the new information is important and is known to be accurate, its influence on the estimated state of the firm will be large, and the investor's estimate of the future profitability of the firm will be greatly affected. If the news is not important, or is thought to be inaccurate, its impact on the a-priori value of the firm will be slight.

To quote Daniel and Titman ([Daniel and Titman, 1999], p. 29) "When rational investors value a stock, they must combine information from a number of sources. For example, they must combine new information with existing information and combine information they collect on their own with information provided by others, such as a company's managers and accountants. Rational investors combine these different sources of information using what is known as Bayes' rule, which specifies that the weights placed on the different pieces of information should be proportional to their respective precision".

In both cases, the investor's perception of the value of the firm, after the news is incorporated, will be affected, and the investor will be left with a different distribution for the firm's state than the one which he or she had before he received the news. If the investor uses the information contained in the news to its maximum, he will also gain a more precise insight as to the condition and future performance of the firm than he or she had before analyzing the news by effectively decreasing the range of most likely values for its state. 
Finally, once the investor has updated his or her view of the value of the firm and estimated its impact on share prices, he or she will then act accordingly by buying or selling the firm's stock. As news arrives into the market, investors repeat the two steps as described, continuously estimating and updating every firm's probability distribution of the state, valuing their stocks, trading accordingly, and thus establishing new stock prices. It is, however, only after these two steps are completed, after investors understand the impact of the news on stocks, that prices are determined.

The process by which investors determine the state of the firm from measurements of its behaviour is very complex and difficult to characterize. In essence, it can be said that investors strive to achieve the best, or the closest to true, image of the firm at any point in time, and thus work at extracting the utmost useful information to establish that picture from the data which they are provided in the form of various measurements of corporations and of the market and economic conditions.

But what is meant by the "closest to true" image of the firm, and how do investors arrive at this type of estimate? These are difficult questions to answer.

As Bachelier states, investors do not realize an exact estimate of the value of the firm (i.e. they are never sure of their estimate) but rather express their knowledge of the firm's value in the form of a probability function, where certain values for the state of the firm are more probable than others. Of course, since each investor looks at the data in different ways, he or she holds his or her own opinions as to the state of the firm, opinions which may differ from that of his or her counterparts. It is, then, based on these estimates, that investors act by buying and selling stock and thus establish prices.

Investors use several tools to extract useful information from the overwhelming amounts of data available to them. The most important tools in the investor's arsenal are his or her own intellect and sense of judgment. These two qualities allow the investor to analyze data and judge situations. The investor can also endow himself or herself with a range of accessory instruments to help him or her in the analysis process. These can take two forms: empirical aids, such as financial models, and expert advice in the form of analyst reports and colleague opinions. Ultimately, however, it is the investor's own thinking process which determines the estimate of the firm's condition by combining the results of his or her personal analysis together with information provided by the accessory instruments. Once an estimate is established, the investor then determines a course of action.

However, since investors have access neither to perfect data nor a flawless model of the firm's behaviour, no amount of analysis nor reasoning will produce 
an error free estimate of the state of the firm - instead, the estimates will contain flaws. For that reason, the estimate of the state is not constituted of a single number, but rather a range of values, thought to most likely characterize the true condition of the company - a probability distribution of the state of the firm. The most precise, or "best", estimate is the one with the smallest range, or, conversely, with the smallest variance.

Ideally, an investor will not stop analyzing the data at his or her disposal until he or she is either satisfied with his or her conclusions, or, is limited in his analysis by either his or her skills or his or her resources, or, in other words, he or she achieves the least variance estimate corresponding to his or her given analytical means. Therefore, ultimately, the qualifier "best" is investor dependent, and an estimate adequate for one investor will not be so for another. An investor's estimate of the state of the firm is thus an expression of a personal perception of the state of nature.

The process just presented can be compared to the concept of filtering widely known in statistics and engineering. In filtering, as in valuing stocks, the researcher is interested in estimating an unknown and unobserved quantity from its measurements, usually corrupted by noise. Models for such problems are usually expressed in the state space form, and solved with the help of the Kalman filter. In the present context, the state represents the value of the firm to be determined from factors such as economic conditions and financial statements and market conditions. Since the problem encountered by investors is in essence an information filtering problem, this study proposes to use the Kalman filter as the solution to the question of extracting the state of the firm from measurements of its condition and for the prediction of stock returns.

The Kalman filter is a recursive estimator which allows for the estimation and prediction of an unknown and unobserved state of a system through sequential weighing of incoming information. The Kalman filter's value lies in its ability to extract information from the sequentially incoming data in order to continuously update the probability distribution of the estimate of the state. The filter achieves this by continuously weighing the accuracy of incoming information with that of the previously estimated states. This process is analogous to investor behaviour, where market participants attempt to estimate the unknown and unobserved state of a system of their interest, namely the firm, by weighing the information value of incoming data with their a-priori knowledge of the condition of the firm. This similarity suggests that the Kalman filter is a mathematical approximation to investor behaviour.

It is important to note that the similarity between engineering and financial 
applications is, however, not perfect. In engineering applications, a model of the behaviour of the phenomenon under study is usually known, while in financial applications, models are rarely specified, and the behaviour of the object under study is rarely perfectly understood.

Let $\mathbf{z}_{i, t}$ be the set of imperfect measurements of the condition of the firm $i$ available to the investor at time $t$ (more precisely $z_{i}(t)$ ). The measurements of the state of the firm can take different forms. For instance, investors may use financial statement data to infer the condition of the firm. Likewise, they may use information from competing firms' statements to examine the relative strength of the firms under analysis. Apart from financial statement information, market participants may use various other sources of information such as news reports, rumours, and external analyst reports to augment their knowledge of the firm. Similarly, they can also use firms' historical stock market performance to estimate the state of the firm. It is also useful to market participants to examine economic data in order to infer the mood of the market and economic prospects. The nature of information used in this study is described in sections 4.3-4.4.

Let $\mathrm{x}_{i, t}$ represent the true, but unknown, value of the state of the firm $i$ (more precisely $x_{i}(t)$ ). The state of the firm represents the collection of all variables describing condition of the firm. The values of these variables are, however, unobservable to the investor, and the investor has to try and determine them from the various measurements available. It takes many variables to describe a system as complex as a firm. For example, a firm's condition could be described through its balance sheet. Similarly, it could be described through management's abilities, or its market share and profile. Ideally, a complete description of the state of a firm should include a combination of all these factors, and the values of these factors have to be estimated from the imperfect measurements of the firm available to the investor. The investor proceeds similarly with respect to the analysis of economic conditions, by analyzing information on the stock market, and the general economy. Thus, the investor's goal is to use the information contained in $\mathbf{z}_{i, t}$ in order to determine the state $\mathrm{x}_{i, t}$ - the future earnings prospects of the firm, in order to make informed investment decisions. The specification of what is meant by the state of the firm and the state of the economy is discussed further in sections $4.3-4.4$.

Formally, the state of a system, $x_{t}$ is "a set of $n$ variables the values of which are sufficient to describe the system behavior completely" [Maybeck, 1979], but is in general, not observable directly. In other words, it is a finite set of numbers which describe the property of interest of a system - in the present case, the object of study is the firm and the property of interest is its condition as an indicator 
of its future profitability.

While the measurements collected by the investor contain valuable information as to the state of the firm, they are not perfect, and could contain errors. At time $t_{j}$, the measurements can be decomposed into two parts: information valuable to the investor in establishing the state of firm $i$, and useless "measurement errors", as follows (the subscript $i$ is omitted for clarity):

$$
z\left(t_{j}\right)=H\left(t_{j}\right) x\left(t_{j}\right)+B_{2}\left(t_{j}\right) u_{2}\left(t_{j}\right)+v\left(t_{j}\right) .
$$

This equation, called the "measurement equation", relates measurements of the condition of firm $i, z_{i}\left(t_{j}\right)$ collected by the investor, to the state of the firm $x_{i}\left(t_{j}\right)$ and the explanatory variables vector $u_{i, 2}\left(t_{j}\right)$, through parameter matrices $H_{i}\left(t_{j}\right)$ and $B_{i, 2}\left(t_{j}\right)$, respectively. The measurement error $v_{i}\left(t_{j}\right)$ represents imperfections, or noise, contained in the available information.

It is important to realize that corporations and the economy are not static entities but evolve in time, and thus, that the state of the firm is not constant, but changes with economic conditions and even because of transformations proper to the firm itself (such as reorganizations, etc...). It can be assumed that the state of the firm cannot change drastically and chaotically from day to day, but rather tends to reflect the changes in the firm and its environment by evolving slowly over time - more precisely, the current situation of the firm depends on its historical circumstances and thus on its past state. In mathematical terms, the model for the temporal evolution of the state of the firm $i$ at time $t_{j}$ can be written as follows (the subscript $i$ is omitted for clarity):

$$
x\left(t_{j+1}\right)=F\left(t_{j}\right) x\left(t_{j}\right)+B_{1}\left(t_{j}\right) u_{1}\left(t_{j}\right)+G\left(t_{j}\right) w\left(t_{j}\right) .
$$

This equation, called the "transition equation" (or state, or, system equation) constitutes a model for the time-propagation of the state of the firm, $x_{i}\left(t_{j}\right)$, and implies that the current state of the firm is a function of its own past. The state is propagated through the parameter matrix $F_{i}\left(t_{j}\right)$ and is affected by a set of explanatory variables $u_{i, 1}\left(t_{j}\right)$ (with parameter $B_{i, 1}\left(t_{j}\right)$ ). Since an exact depiction of the evolution of the state is impossible, the model compensates for this through the addition of "transition errors", $w_{i} t_{j}$ - imperfections in investors' model for the time-evolution of the firm's state.

Together equations 4.1 and 4.2 represent a state space model for the behaviour of the firm. The state space representation of the firm at any point in time is thus:

$$
\begin{aligned}
z\left(t_{j}\right) & =H\left(t_{j}\right) x\left(t_{j}\right)+B_{2}\left(t_{j}\right) u_{2}\left(t_{j}\right)+v\left(t_{j}\right), \\
x\left(t_{j+1}\right) & =F\left(t_{j}\right) x\left(t_{j}\right)+B_{1}\left(t_{j}\right) u_{1}\left(t_{j}\right)+G\left(t_{j}\right) w\left(t_{j}\right) .
\end{aligned}
$$


In order to successfully predict returns using this framework, it is necessary to specify the information set $\mathbb{z}_{i, t}$, the structure of the state of the firm $x_{i, t}$, the sets of explanatory variables $u_{i, 1}(\cdot)$ and $u_{i, 2}(\cdot)$, and the parameter matrices $H_{i}(\cdot), F_{i}(\cdot)$, $B_{i, 1}(\cdot)$, and $B_{i, 2}(\cdot)$, and assumptions about the statistical nature of the noises $v_{i}$ and $w_{i}$. Finally, to complete the implementation, it is necessary to find a method, similar to the one used by investors, for estimating the state of the firm $x_{i, t}$ from the information set $\mathbf{z}_{i, t}$.

That state space modelling approach differs from the usual linear regression approach. In the regression setting, the emphasis is on finding a relationship between the dependent and independent variables by estimating the regression parameters. In state space modelling, ones uses measurements to estimate the value of an unobserved state variable whose behaviour is described by the state equation. It should be noted that it is possible to formulate a regression problem in the state space framework, but that the reverse is not true.

Successful investments require investors to continuously update their estimates of the state of the firm whenever new information arrives. Since investors' estimates are represented by probability functions, this process amounts to updating the probability distribution function of the state of the firm using measurements, and represents essentially a Bayesian procedure. This assumption may represent a deviation from actual behaviour, but is mathematically convenient. Denote by $f\left(x_{t} \mid Z_{t}\right)$ the prior probability distribution of the state of the firm $x$ at time $t$ conditional on all information available to the investor about the firm up to and including time $t\left(Z_{t}=\left\{z_{t}, z_{t-1}, z_{t-2}, \ldots, z_{0}\right\}\right)$. This distribution represents the investor's total a-priori knowledge of the firm state before processing any additional measurements on the firm or the market.

Since the investor's goal is to assess with the highest precision the state of the firm at any point in time, whenever news arrives, he or she must update his or her estimate of the state of the firm $x_{t}$ to reflect the new information. In other words, once a new measurement $z_{t+1}$ appears, he or she updates the probability distribution function for the state of the firm $x_{t+1}$. The process thus consists of two steps: propagation and updating.

First: before receiving any new information about the firm, the investor estimates the future expected condition of the firm based solely on his knowledge of the firm's dynamics. This estimate represents the investor's expectation as to the future state of the firm. In this step, the investor in fact establishes a distribution function of the state of the firm at time $t+1$ given information up to and including time $t$ only: $f\left(x_{t+1} \mid Z_{t}\right)$. This distribution function is derived based on investor's time $t$ knowledge of the state of the firm (in the form of $f\left(x_{t} \mid Z_{i}\right)$ ), and the in- 
vestor's model of firm dynamics as represented by the state transition equation 4.2 .

Second: whenever a new piece of information, $z_{t+1}$, arrives, the investor immediately refreshes his or her estimate of the state of the firm. This step consists of updating the guess as to the state of the firm $x_{t+1}$ based on old information, $Z_{t}$, with the information recently received, and consists in formulating a new probability function for the state, $f\left(x_{t+1} \mid Z_{t+1}\right)$. The desired probability distribution is estimated through the knowledge of the measurement model of the firm as expressed via the measurement equation 4.1 . The investor uses this updated probability function as the basis for further investment analysis. The two steps are repeated as new information arrives, the first yielding forecasts of the future condition of the firm and the second yielding updated estimates of its state.

In valuing stocks, investors combine fresh news together with their a-priori knowledge of the corporation into a "best" estimate of the condition of the firm. Since their estimates are probability distributions of the state of the firm, each time new information is received investors update these distributions so as to reflect the new state of nature. Investors thus essentially perform a Bayesian updating process. Assuming that investors look for the most precise estimate of the firm's state, the solution to the problem of state estimation exists in the form of the Kalman filter. Under the assumption that the errors $v_{i, t}$ and $w_{i, t}$ are normally distributed, and the model's functions, $H(\cdot)$, and, $F(\cdot)$, are linear, the Kalman filter computes the linear minimum mean square error estimate of the state $\mathbf{x}_{i, t}$ given the information set $\mathbf{z}_{i, t}$ (see Maybeck p. 232 [Maybeck, 1979]). For the lack of an exact model of the investor's thinking process, it is believed an estimate of the state of the firm obtained from the Kalman filter is a fair approximation to the Bayesian estimate of the condition of the firm obtained by investors using nothing more than their experience and reasoning. As the Kalman filter procedure can also be used to predict the state of the firm, it is assumed to be a fair representation of the expectation forming process used by investors.

It is important to note that the above is only a framework, or approximation, of investor behavior, and that investors do not possess an explicit valuation model, but rather demonstrate an implicit behavior that can be described with the above equations. It is also important to stress that there is no one-to-one mapping between the engineering concept of Kalman filtering and the financial concept of information analysis. It is believed, however, that the financial analysis process followed by investors can be approximated by the concept of filtering. As such, it is believed that the method presented here will enable the models to extract relevant information about the state of the markets from the stream of data in 
approximately the same manner as a real investor would.

In order to estimate the state of a firm, investors analyze the data available to them according to a certain valuation model, and produce estimates of the state. Assuming that investors seek a minimum error estimate of the state of the firm, it is believed that the Kalman filter represents a fair approximation to investor's estimating behavior, allowing one to estimate the state of a system in situations where perfect information is not available, and thus emulating real market conditions. The Kalman filter is thus the principal methodology used in the current study.

\subsection{A Univariate Model of Stock Returns}

This section develops a univariate model for the prediction of stock returns. The proposed model describes the behavior of the daily state of the firm based on measurements of the stock's past performance as expressed by stock returns. The univariate model tests the weak form of market efficiency hypothesis by modelling the behavior of stock returns using their past history alone. The goal of the model is to extract the state of the firm from observations of past stock performance, and, on that basis, to predict the future behavior of the firm's returns and ultimately to construct a trading rule for exploiting abnormal profit opportunities.

A market is said to be efficient if it is impossible to construct a trading rule based solely on historical information that would yield abnormal excess returns. If it is possible to construct such a rule, the market is said to be inefficient, since prices do not reflect all relevant information. Fama [Fama, 1970, Fama, 1991] has defined three types of efficiency, based on the type of information used by investors in establishing price forecasts. Based on that classification, the current study, with the univariate and multivariate models, represents, respectively, a test of the weak and semi-strong forms of efficiency.

A test of market efficiency is a delicate matter. For a market to be efficient, it is not only necessary that prices be unpredictable, but, more importantly, that there be no excess profit opportunities from using past data to predict the market. This means that a certain level of predictability in prices will still be consistent with an efficient market, as long as the profits from exploiting this predictability will not exceed transaction costs. Ultimately, the only test of efficiency is a profitability test.

The study attempts two tests of efficiency in the guise of two predictive models. The univariate model represents a test of the weak form of efficiency, while 
the multivariate model represents a test of the semi strong form of efficiency. Because the study does not use all publicly available information, but rather easily available information, the study is in fact a data based abstraction of the tests of market efficiency. This does not, however, invalidate the study, since the discovery of a profitable trading rule based on the limited dataset will still lead to a rejection of either of the forms of market efficiency.

Each of these two tests represents a test of market efficiency against a specific alternative. In the univariate model case, the test verifies that returns can be predicted using historical price records alone, and in the multivariate model case, that they can be predicted using historical returns records as well as a select group of economic variables. If the models' results can be translated into profitable trading rules (after risk adjustment), then the market efficiency hypothesis will be rejected. An unfavourable result, however, will only mean that the alternative models proposed herein are not strong enough to reject the null hypothesis of market efficiency.

At this point in the discussion, it is essential to discuss the scope of the proposed framework. As already mentioned, the framework introduced above represents a model of investor behaviour in valuing stocks. The framework does not specify, however, which specific investor's behaviour it describes - indeed, the system is so general that it proposes that the behaviour of any and all investors participating in the market can be described within it. In the grand scheme of things, every market participant possesses his or her own model for analyzing and interpreting data, and for each of these investors, it is proposed that his or her behaviour can be approximated with the help of the already mentioned framework.

The objective of this work is to test market efficiency with the help of a new approach to forecasting stock performance, an approach based on a specific model of investor behaviour. The test will consist of finding models, within the specified framework, to accurately estimate the state of the various firms in the market so as to predict their future performance.

Once established, a model of this type will in fact represent a single market participant. The question is, which participant does the model represent? This is a difficult question to answer. It could be argued that because the study concentrates on the use of daily, end-of-day data, that, for this reason alone, the investor in question must be the market maker. Indeed, he or she is probably the investor able to make successful end-of-day trades. This question is, however, open to discussion.

To construct a state space model for the prediction of returns one must first answer the question, "What is the state of the firm?" In order to answer this 
question one has to consider its context: having access only to past prices as measurements of the condition of the firm (in the basic model), the state is the smallest set of information describing adequately their behavior.

Even though the stock price tends to reflect the value of the firm, not every change in the price of the stock represents a change in the value of the firm. The reason being, that the markets are not necessarily perfect. By gathering and analyzing all types of information, investors formulate realistic guesses as to the future profitability of the firm and discount them into the present value, or the condition, or the state of the firm.

Since not every piece of information is relevant or interpreted correctly, and does not necessarily affect the value of the firm, but may affect its price, two components to the state of the firm should be considered: the underlying value of the firm and transitory shocks unrelated to it such as market psychology. In other words, stock returns can be said to have two components: fundamental and nonfundamental, where the fundamental component of the stock return represents the change in the stock's true value, while the non-fundamental part represents temporary deviations from the true underlying value of the firm. It is on the basis of these two unobserved components that investors make investment decisions. Unfortunately, neither of these components is directly observable by market participants - only stock changes being known. In order to make an investment decision, investors, using their intuition and knowledge of the markets, need to estimate these two components from the data available to them. The univariate model of returns attempts to mimic the estimating behavior of market participants through artificial empirical means, by concentrating only on the use of data consistent with tests of the weak form of market efficiency.

\subsubsection{Measurement Equation}

Let $r_{i, t}=\left(P_{i, t}-P_{i, t-1}\right) / P_{i, t-1}$ for firm $i$ during day $t$. The observed returns $r_{i, t}$, represent the investor's measurements of the firm's performance, and the investor's only input into his or her model for determining the condition of the firm (i.e. $\mathbf{z}_{i, t} \equiv r_{i, t}$ are the only measurements available to the investor are assumed here to be historical returns).

Let $r_{i, t}^{\text {fund }}$ and $r_{i, t}^{\text {nfund }}$ represent respectively the fundamental and non-fundamental components of the stock returns. The measured returns can be considered to be the sum of these two components: $r_{i, t}=r_{i, t}^{\text {fund }}+r_{i, t}^{\text {nfund }}$. The univariate model stipulates the following representation for the measurement model of the individual 
stocks:

$$
r_{i, t}=r_{i, t}^{\text {fund }}+r_{i, t}^{\text {nfund }}+v_{i, t}
$$

where the observed return on stock $i$ on day $t$ is expressed as the sum of the two individual return components representing the state of the firm, and an error term representing the errors that arise in observing the performance of the firm.

\subsubsection{Firm Transition Equation}

The first step in the specification of a transition equation for the state of the firm consists of determining the nature of the behavior of the fundamental part of returns. Many ad-hoc models have been proposed in the literature to explain the behaviour of returns, however, it is believed that none of them reflect it adequately. Fundamental returns are a reflection of the information about the growth of the firm contained in stock returns, and therefore in order to specify a model for their behavior, it is necessary to know how firms grow.

It is proposed here that the fundamental component of retums follows a damped linear trend model, first introduced by Harrison and Stevens

[Harrison and Stevens, 1976], where the variable follows a time varying linear stochastic trend. This model allows fundamental returns to vary in two different ways: 1) they can be constant over time, or 2) they can follow a stochastic linear trend over time. Additionally, this model is tractable in the state space framework [Harvey, 1989]. The linear growth model of the fundamental component of stock returns, $r_{i, t}^{\text {fund }}$, is:

$$
\begin{aligned}
r_{i, t}^{\text {fund }} & =r_{i, t-1}^{\text {fund }}+g_{i, t-1}+\eta_{i, t}^{(1)} \\
g_{i, t} & =\rho g_{i, t-1}+\eta_{i, t}^{(2)},
\end{aligned}
$$

where $g_{i, t}$ represents the growth rate in the fundamental return, and the $\eta^{(*)}$ are uncorrelated white noise disturbances with variances, respectively $\sigma_{\eta^{(1)}}$ and $\sigma_{\eta^{(2)}}$. The damping parameter $0 \leq \rho \leq 1$ allows predictions for fundamental returns to level off with time, and thus does not produce exaggerated level forecasts. Appendix $C$ provides more information on the univariate model.

The literature has shown the day of the week effect to be a persistent and unexplained anomaly in the behavior of stock returns [French, 1980]. The principal findings of this literature are that Monday returns tend to be the lowest in the week and negative, and Friday's returns tend to be the highest. This finding suggests that including a day-of-the-week seasonal variable in the model for the 
behavior of returns may improve predictive performance. Since the day of the week effect has not been explained yet by either institutional or other factors, it can be considered as a psychological effect, and as such can be considered as part of the non-fundamental component of returns: $r_{i, t}^{\text {nfund }}=d_{t}+r_{i, t}^{\mathbf{I} *}+\epsilon_{i, t}$, where $d_{t}$ is the seasonal effect related to time $t$, and $r_{i, t}^{I *}+\epsilon_{i, t}$ represents the part of the non-fundamental component of returns that is unrelated to the day of the week effect.

While the day-of-the-week effect is important in explaining the behavior of stock returns, it is not the only psychological influence affecting them, there being other factors having an effect on an investor's expectation forming process. It is proposed here that the other influences, and thus remaining part of the nonfundamental component of returns, can be modelled through an ARMA process. It is assumed, for tractability reasons, that the psychological factors affecting the behaviour of returns follow a stationary process.

The complete state space specification for the univariate model of returns is as follows. The measurement equation is:

$$
r_{i, t}=r_{i, t}^{\text {fund }}+r_{i, t}^{\text {nfund }}+v_{i, t}
$$

The transition equation is:

$$
\begin{aligned}
r_{i, t}^{\text {fund }} & =r_{i, t-1}^{\text {fund }}+g_{i, t-1}+\eta_{i, t}^{(1)} \\
g_{i, t} & =\rho g_{i, t-1}+\eta_{i, t}^{(2)} \\
r_{i, t}^{\text {nfund }} & =d_{t}+r_{i, t}^{\mathrm{I} *}+\epsilon_{i, t}
\end{aligned}
$$

where $r_{i, t}^{\text {fund }}$ follows a non-stationary damped linear trend model, $d_{t}$ is the day of the week effect, and $r_{i, t}^{\mathrm{I} *}$ represents the remaining part of the non-fundamental component of stock returns which follows an $\operatorname{ARMA}(p, q)$ process. For the complete description of the model, the reader is directed to Appendix C.

\subsection{The Multivariate Model}

Investors who analyze investment opportunities have to look not only at the fundamental factors affecting the performance of individual firms, but also at the broad market and economic conditions. In order to identify successful investments, investors need to estimate the present and the likely future conditions of the markets and the economy, together with their probable influence on the performance of 
the individual stocks. This task is performed through a thorough analysis of financial, economic and other qualitative data, and results in formation of financial forecasts. Chan, Kacerski and Lakonishok [Chan et al., 1998] classify factors affecting stock returns into five categories:

1. fundamental factors, which represent firm specific variables, primarily accounting variables,

2. technical factors borrowed from the technical analysis literature,

3. macro-economic factors reflecting the state of the economy, such as interest rates, money supply, industrial production, etc ...

4. market factors describing the condition of the stock market, mainly through stock market averages, and,

5. statistical factors based on statistical analysis of historical returns.

In their day-to-day activities, however, investors do not have the luxury of performing analysis using all these factors because of the simple lack of immediate and accurate data. Indeed, detailed analysis can only be carried out when data is both timely and in adequate supply - since most economic and financial data are not collected nor released on a daily basis, but rather at weekly or monthly intervals, the investor, on a day-to-day basis, does not have access to the majority of the above mentioned factors, and must therefore use the little data that is at his or her disposal. The data investors acquire on a daily basis are mostly financial data composed of observations on the performance of the stock and bond markets and subjective data from news feeds.

The day-to-day market analysis and prediction are therefore, for the lack of data, rather challenging tasks, and the literature does not seem to contain many attempts at daily retum prediction. Nevertheless, investors seem to thrive in this atmosphere where rumours and information intermingle. This section proposes a model for the prediction of the individual stock returns in a multivariate setting.

Stock returns are recognized to be composed of two components: systematic and unsystematic. The univariate model is a prediction system based on the unsystematic component of returns - it predicts returns based only on the firm's own past performance and represents a test of the weak-form efficient market hypothesis. The multivariate model of returns proposes to forecast returns by modelling both the systematic and unsystematic component of returns, i.e. to predict returns based on both, the firm's past performance and on economic factors. Let $X_{i, t}^{S}$ and 
$X_{t}^{M}$ be respectively the state of the firm and the state of the economy. Then the full model proposes that the stock return for firm $i$ at any time $t, r_{i, t}$, is a function of both, the state of the firm (it's financial condition, earnings prospects, management talent etc ...), and the state of the market (general market performance, interest rates, inflation etc ...) as follows:

$$
r_{i, t}=h\left(X_{i, t}^{S}, X_{t}^{M}\right)+v_{i, t} .
$$

It is assumed for convenience that the states of the market, $X_{t}^{M}$, and the firm, $X_{i, t}^{S}$, are additive in nature, i.e. that the above relation can be rewritten as:

$$
r_{i, t}=h_{1}\left(X_{i, t}^{S}\right)+h_{2}\left(X_{t}^{M}\right)+v_{i, t}
$$

The assumption of the separability or additivity of the two states is made for tractability reasons, but can be argued to be a reasonable simplification. The state of the firm can be estimated by looking at its financial statements, business practices, management and other aspects, at any point in time, regardless of the state of the economy. The same can be said for the state of the economy: one can estimate it based on broad business and economic statistics without having to look at every firm composing it. While the economy surely affects the performance of individual firms, it tends to do so slowly, over the medium and long terms. Economic policy and news rarely provoke sudden changes in the performance of the firms themselves other than creating sudden one-time ripples in the market.

The disassociation of the two states will ease the computational burden, by allowing one to compute a unique state of the economy separately from the state of the individual firms. Letting the state of the economy and the market, $X_{t}^{M}$, be distinct from the state of the firm, $X_{i, t}^{S}$, allows for a breakdown of the model into two parts: a market sub-model and a firm sub-model. In this setup, the market submodel estimates the state of the market, while the firm sub-model estimates the state of the firm. This framework effectively delegates the task of estimating the state of the market to a separate model, which allows the use of a single estimate of the state of the market in all the individual firm forecasting models.

In order to predict the behavior of the market, it is important to characterize the state of the stock market $x_{t}^{\mathrm{M}}$, which consists of the most important pieces of information describing its condition and behavior. To make investment and valuation decisions on a daily basis, investors estimate the state of the firms and the economy around them by concentrating on certain key factors affecting the behavior of both systems. They can be classified into two categories: factors related to the performance of the stock market, and factors related to the behavior of interest 
rates. The first category describes the behavior and condition of the stock market in general as well as the health of firms, while the second category describes the general state of the economy. Adding to this list, the literature also uses several measures of general economic condition such as the money supply, industrial production, and, GNP. Unfortunately, the unavailability of these measures of economic performance on a daily basis prevents their use in the day-to-day workings of investors, and disqualifies them from this study. The Data Appendix A contains more details on the nature and sources of the data used in the study.

In the "global" economy, stock values depend on many influences, some of which are international, and in describing the state of the markets it is necessary to account for them. It is assumed that investors observe the behavior of three markets: the American market as represented the DJ Composite Average, the UK stock market representing Europe described by the FTSE 100 index, and the Japanese Market described by the Nikkei 225 stock market index. Denote the percentage returns from these markets respectively as $r_{t}^{\mathrm{US}}, r_{t}^{\mathrm{E}}$ and $r_{t}^{\mathrm{J}}$.

Additionally, investors rely on the behaviour of exchange rates for the US, UK and Japan. Let $s_{t}^{\text {USE }}$ and $s_{t}^{\text {US.JP }}$ be respectively returns in the US/Pound and the US/Japan exchange rates, and let $s_{t}^{\mathrm{EJP}}$ be the change in the implied cross exchange rate for the Pound and the Yen. The interest rate measurements used by investors include percentage changes in the term premium, $n_{t}^{\mathrm{LT}}$ (defined as the difference between short and long bond rates), percentage changes in the risk premium, $n_{t}^{\text {Rsk }}$ (the difference between low and high risk bonds), and the percentage changes in the t-bill rate $n_{t}^{f}$.

The measurements of the state of the economy and the markets consist of returns on the international stock markets, changes in interest rates and changes in exchange rates. For tractability reasons, it is assumed that these components can be modelled through an autoregressive model. Using this information, the investor seeks to estimate the state of the markets.

Given the assumption that the state of the market can be modelled as an autoregressive model, the complete model for the behaviour of the market is as follows. The market measurement model and the transition (state) equation for the state of the market are presented respectively in equations 4.12 and 4.13 . 


\begin{tabular}{|c|c|c|c|c|c|c|c|c|}
\hline 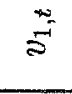 & $\overrightarrow{\mathbf{v}}$ & $\stackrel{+}{5}$ & $\overrightarrow{a^{*}}$ & $\vec{b}$ & $\vec{s}$ & $E$ & $\overrightarrow{a^{\circ}}$ & $\stackrel{s}{s}$ \\
\hline \multicolumn{9}{|c|}{+} \\
\hline$\vec{\omega}$ & $\vec{w}$ & $\vec{n}$ & $\vec{w}$ & $w_{n}^{*}$ & $\vec{\omega}$ & in & $w_{n}^{+\infty}$ & $\ddot{\omega}$ \\
\hline \multicolumn{9}{|c|}{$\times$} \\
\hline 0 & 0 & 0 & 0 & 0 & 0 & 0 & 0 & -1 \\
\hline 0 & 0 & 0 & 0 & 0 & 0 & 0 & - & 0 \\
\hline 0 & 0 & 0 & 0 & 0 & 0 & - & $=$ & 0 \\
\hline 0 & 0 & 0 & 0 & 0 & -1 & 0 & 0 & 0 \\
\hline 0 & 0 & 0 & 0 & -1 & 0 & 0 & 0 & 0 \\
\hline 0 & 0 & 0 & -1 & 0 & 0 & 0 & 0 & 0 \\
\hline 0 & 0 & - & 0 & 0 & 0 & 0 & 0 & 0 \\
\hline 0 & - & 0 & 0 & 0 & 0 & $=$ & 0 & 0 \\
\hline$\rightarrow$ & 0 & 0 & 0 & 0 & 0 & 0 & 0 & 0 \\
\hline \multicolumn{9}{|c|}{$\|$} \\
\hline$\underbrace{}_{\infty}$ & $\begin{array}{l}\underbrace{}_{D^{2}} \\
D_{\infty}\end{array}$ & 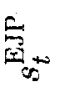 & 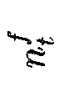 & 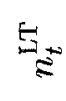 & ثָ & $\vec{s}_{\infty}$ & 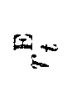 & $=$ \\
\hline
\end{tabular}




\begin{tabular}{|c|c|c|c|c|c|c|c|c|}
\hline 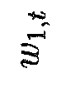 & $\stackrel{\sim}{\tilde{\Sigma}}$ & 今 & $\stackrel{\Xi}{\Xi}$ & $\frac{\sqrt[4]{5}}{3}$ & 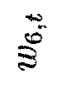 & $\stackrel{\overrightarrow{5}}{\stackrel{5}{5}}$ & 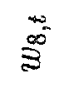 & $\stackrel{5}{5}$ \\
\hline \multicolumn{9}{|c|}{+} \\
\hline$\stackrel{I}{=}$ & $\underset{i}{i}$ & $\stackrel{i}{i}$ & $\stackrel{1}{5}$ & $\begin{array}{l}3 \\
3 \\
5 \\
2\end{array}$ & $\begin{array}{l}1 \\
1 \\
\text { wi }\end{array}$ & $\frac{7}{5}$ & $\underbrace{i}$ & i \\
\hline \multicolumn{9}{|c|}{$x$} \\
\hline 0 & 0 & 0 & 0 & 0 & $\theta$ & 0 & 0 & 8 \\
\hline 0 & 0 & 0 & 0 & 0 & 0 & 0 & 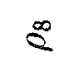 & 0 \\
\hline 0 & 0 & 0 & 0 & 0 & 0 & $\varepsilon$ & 0 & 0 \\
\hline 0 & 0 & 0 & 0 & 0 & $\varepsilon$ & 0 & 0 & 0 \\
\hline 0 & 0 & 0 & 0 & 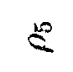 & 0 & 0 & 0 & 0 \\
\hline 0 & 0 & 0 & $\Xi$ & 0 & 0 & 0 & 0 & 0 \\
\hline 0 & 0 & 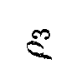 & 0 & 0 & 0 & 0 & 0 & 0 \\
\hline 0 & $\varepsilon$ & 0 & 0 & 0 & 0 & 0 & 0 & 0 \\
\hline$\Sigma$ & 0 & 0 & 0 & 0 & 0 & 0 & 0 & 0 \\
\hline \multicolumn{9}{|c|}{$\|$} \\
\hline$\stackrel{+}{=}$ & $\stackrel{\vec{v}}{w}$ & $\vec{s}$ & $w^{*}$ & $\vec{w}$ & in & $\stackrel{+}{N}$ & $\omega_{\infty}^{+\infty}$ & $\vec{\omega}$ \\
\hline
\end{tabular}


The model 4.12-4.13, models stock market returns, foreign exchange returns as well as percentage changes in selected interest rates as multiple $\mathrm{AR}(1)$ models. The vectors $V_{t}^{\mathrm{Mkt}}=\left[v_{1, t}, v_{2, t}, v_{3, t}, v_{4, t}, v_{5, t}, v_{6, t}, v_{7, t}, v_{8, t}, v_{9, t}\right]^{T}$ and $W_{t}^{\mathrm{Mkt}}=\left[w_{1, t}, w_{2, t}, w_{3, t}, w_{4, t}, w_{5, t}, w_{6, t}, w_{7, t}, w_{8, t}, w_{9, t}\right]^{T}$ represent respectively measurement and state estimation errors, and have variances: $\operatorname{Var}\left(V_{t}^{\mathrm{Mkt}}\right)=R_{t}^{\mathrm{Mkt}}$, and $\operatorname{Var}\left(W_{t}^{\mathrm{Mkt}}\right)=Q_{t}^{\mathrm{Mkt}}$. It is assumed for tractability that the residuals are uncorrelated between themselves.

The goal of the multivariate model is to better predict individual stock returns by accounting for the systematic market factors influencing stock returns. Now that a model has been proposed to explain the behavior of the broad market, it is necessary to include it in the univariate model for prediction purposes. Originally, the general form of the univariate model proposed in the previous section was:

$$
\begin{aligned}
r_{i, t} & =r_{i, t}^{\text {fund }}+r_{i, t}^{\text {nfund }}+v_{i, t}, \\
r_{i, t}^{\text {fund }} & =r_{i, t-1}^{\text {fund }}+g_{i, t-1}+\eta_{i, t}^{(1)} \\
g_{i, t} & =\rho g_{i, t-1}+\eta_{i, t}^{(2)} \\
r_{i, t}^{\text {nfund }} & =d_{t}+r_{i, t}^{\mathrm{I} *}+\epsilon_{i, t} .
\end{aligned}
$$

In order to account for economic factors in the behaviour of returns, the multivariate model uses the estimated state of the markets to explain observed stock returns. This is achieved by including the state of the market as computed by the market sub-model as an explanatory variable in the univariate model of returns - in essence, the state of the market as computed by the market model serves as an input into the univariate model. The general representation of the multivariate model is as follows. The measurement model:

$$
r_{i, t}=r_{i, t}^{\text {fund }}+r_{i, t}^{\text {nfund }}+\beta^{\mathrm{Mkt}} \hat{Z}_{t}^{\mathrm{Mkt}(-)}+v_{i, t} .
$$

The state transition equations:

$$
\begin{aligned}
r_{i, t}^{\text {fund }} & =r_{i, t-1}^{\text {fund }}+g_{i, t-1}+\eta_{i, t}^{(1)} \\
g_{i, t} & =\rho g_{i, t-1}+\eta_{i, t}^{(2)} \\
r_{i, t}^{\text {nfund }} & =d_{t}+r_{i, t}^{\mathrm{I} *}+\epsilon_{i, t},
\end{aligned}
$$

where $\hat{Z}_{t}^{\mathrm{Mkt}(-)}$ is the predicted state of the market at time $t$, and $\beta^{\mathrm{Mkt}} \hat{Z}_{t}^{\mathrm{Mkt}(-)}$ represents the influence of the state of the economy on stock returns. More pre- 
cisely, the multivariate model's measurement equation is:

$$
\begin{aligned}
r_{i, t} & =r_{i, t}^{\text {fund }}+r_{i, t}^{\text {nfund }}+\beta^{\mathrm{Mkt}} \hat{z}_{t}^{\mathrm{Mkt}(-)}+v_{i, t} \\
& =r_{i, t}^{\text {fund }}+r_{i, t}^{\text {nfind }} \\
& +\beta^{1} s_{t}^{\mathrm{USE}}+\beta^{2} s_{t}^{\mathrm{USJP}}+\beta^{3} s_{t}^{\mathrm{EJP}}+\beta^{4} n_{t}^{f} \\
& +\beta^{5} n_{t}^{\mathrm{LT}}+\beta^{6} n_{t}^{\mathrm{Rsk}}+\beta^{7} r_{t}^{\mathrm{US}}+\beta^{8} r_{t}^{\mathrm{E}}+\beta^{9} r_{t}^{\mathrm{J}}+v_{i, t}
\end{aligned}
$$

where $\beta^{*}$ are parameters, and $s_{t}^{\mathrm{USE}}, s_{t}^{\mathrm{USJP}}, s_{t}^{\mathrm{EJP}}, n_{t}^{f}, n_{t}^{\mathrm{LT}}, n_{t}^{\mathrm{Rsk}}, r_{t}^{\mathrm{US}}, r_{t}^{\mathrm{E}}, r_{t}^{\mathrm{J}}$ are the predicted exchange rate returns, predicted changes in the interest rates and the predicted values of stock index returns. The predictions for the appropriate time period are obtained from the market sub-model 4.12-4.13.

\subsection{The proposed models and the literature}

The study proposes two models for the behavior of returns. This section briefly discusses the differences between these models and those present in the literature.

In the literature on returns forecasting, analysis is performed on the basis of the time-series behavior of returns with the help of simple linear models. The literature assumes that stock returns are a function of the rational behavior of market participants and does not attempt to to dissect them into more elementary components. As such, standard time series methodologies are used to describe the behavior of returns.

The univariate model proposed in this study, on the other hand, assumes that returns can be decomposed into more fundamental components. In particular, it is assumed that returns can be decomposed into a component due to rational investor behavior and a component due to investors' psychological biases. The structural nature of this model makes it natural for it to be inscribed in the state-space framework and treated with the help of the Kalman filter. While models featuring the Kalman filter have been used in the literature previously (see for example Conrad and Kaul [Conrad and Kaul, 1988]), they have been used in simple time series studies that have not fully exploited the power of the state-space form to describe the nature of stock returns.

The multivariate model of returns proposed here differs from the standard multivariate studies found in the literature as well. The standard approach in the multivariate forecasting of returns uses multiple regression (see for example Pesaran and Timmerman [Pesaran and Timmerman, 1994]). The use of the state-space 
framework allows the current study to model returns by defining a model for the behavior of the market and combining it with the univariate model to yield better predictions.

\subsection{Prediction and Testing}

\subsubsection{The Data}

The study concerns daily predictability of returns for 10 stocks selected from among the 30 stocks composing the Dow Jones Industrial Average as of January 1, 2000. The DJIA index is a collection of the 30 most important firms traded on American markets. Data on these stocks is available for the period of January 1 1990 to January 12000 . Appendix A contains further information about the data.

Individual stock data is composed of split and dividend adjusted stock prices. Raw returns $r_{i, t}$, for firm $i$ at time $t$ are computed from these data as the percentage changes in price from market close on one day to the market close on the following day.

In the current study, the DJ Composite index is used as a proxy for the complete US market, while for Europe and Japan, the British FTSE100 and the Nikkei 225 stock market indices respectively have been selected as proxies. The study defines $r_{t}^{\mathrm{US}}$ as the return from market close on day $t-1$ to close on day $t$ on the DJ Composite index, and $r_{t}^{\mathrm{E}}$ and $r_{t}^{\mathrm{J}}$ as respectively the daily returns on the FTSE100 and the Nikkei 225 stock market indices.

The interest rates represent another measurable aspect of the state of the economy. We define the nominal interest rate $n_{t}^{f}$ as the level of the 3-month T-Bill rate. The long term interest rates $n_{t}^{\mathrm{LT}}$ are measured as the yields on 30-year Bonds, and high risk bonds $n_{t}^{\text {Rsk }}$ are measured as the yields on Moody's BAA corporate bonds.

Foreign exchange returns for the three most important economies are also measured at daily intervals. The exchange rates are defined as follows: $s_{t}^{\text {USE }}$ represents the return on the $£$-US exchange rate, $s_{t}^{\text {USJP }}$ those on the US- rate, and $s_{t}^{\mathrm{EJP}}$ the returns on the implied exchange rate between $£$ and $¥$. Foreign exchange data are measured as the number of foreign currency units per one US dollar. The data consist of two sets of measurements: the $\mathscr{L} / \mathrm{US}$, and $¥ / \mathrm{US}$ exchange rates. The implied $£ /$ foreign exchange rate is computed as follows:

$$
S_{t}^{£ / \#}=\frac{\mathfrak{E}_{\mathrm{t}}}{\mathrm{US} \$_{t}} \cdot \frac{\mathrm{US} \$_{t}}{\Psi_{t}}
$$


Foreign exchange returns are computed similarly to the returns on individual stocks: $s_{t}^{\mathrm{k}}=\left(S_{t}^{\mathrm{k}}-S_{t-1}^{\mathrm{k}}\right) / S_{t-1}^{\mathrm{k}}$, where $k \in\{£ / \$, \Psi / \mathbb{\$} / \Varangle\}$. The Data Appendix A presents more information on the foreign exchange data.

\subsubsection{Methodology}

The Kalman filter is the principal methodology used in the present study. This section presents a short summary of the technicalities of the method which yields predictions of the unobservable state of the system given the latest information. The reader is further referred to the vast literature on Kalman filtering present in the statistical and engineering fields, most importantly Harvey [Harvey, 1989] and Meinhold and Singpurwalla [Meinhold and Singpurwalla, 1983].

The Kalman filter is a method for estimating the state of a system using measurements of its behavior. Let $z_{t}$ denote the measurement stemming from a system of interest, and let $x_{t}$ be the state of that system. As previously stated, a state space model consists of two equations, a measurement equation,

$$
z_{t}=H_{t} x_{t}+B_{2, t} u_{2, t}+v_{t}
$$

relating the state of the system to observations of its behavior, and a state equation,

$$
x_{t+1}=F_{t} x_{t}+B_{1, t} u_{1, t}+G_{t} w_{t}
$$

describing the evolution of the system's state in time. The errors $v_{t}$ and $w_{t}$ represent respectively imperfections in the measurements of the system's behavior, and the errors in modelling its state, and are called measurement and estimation disturbances. The errors have zero means, are mutually uncorrelated and have variances respectively: $\operatorname{Var}\left(v_{t}\right)=R_{t}$, and $\operatorname{Var}\left(w_{t}\right)=Q_{t}$.

Denote the initial estimate of the state as $\hat{x}_{t}$ and its variance as $P_{t}$ (for this derivation, assume that the inputs $u_{1}\left(t_{i}\right)$ and $u_{2}\left(t_{i}\right)$ are zeros) Then, the estimate of the state at time $t+1$ based on information available at time $t$, and its variance can be written as:

$$
\begin{array}{r}
\hat{x}_{t+1}^{-}=F_{t} \hat{x}_{t}, \\
P_{t+1}^{-}=F_{t} P_{t} F_{t}^{T}+G_{t} Q_{t} G_{t}^{T} .
\end{array}
$$

Once a new measurement arrives at time $t+1$, the estimate of the state of the system can be updated by discounting the new information it brings with the information already contained in the model through the updating equations:

$$
\hat{x}_{t+1}^{+}=\hat{x}_{t+1}^{-}+K_{t+1}\left[z_{t+1}-H_{t+1} \hat{x}_{t+1}^{-}\right]
$$




$$
\begin{aligned}
P_{t+1}^{+} & =P_{t+1}^{-}-K_{t+1} H_{t+1} P_{t+1}^{-} \\
K_{t+1} & =P_{t+1}^{-} H_{t+1}^{T}\left[H_{t+1} P_{t+1}^{-} H_{t+1}^{T}+R_{t+1}\right]^{-1}
\end{aligned}
$$

and where $K_{t+1}$ is referred to as the Kalman gain. Once a model is formulated, the Kalman filter can be used for the purposes of state estimation and the state prediction.

The Kalman filter allows its user to estimate the state of a system described through equations in state space form. In order to estimate the state however, the practitioner must have full knowledge of model parameters $H_{t}, F_{t}, R_{t}$ and $Q_{t}$. Unfortunately, in most cases the parameter matrices are not known, and only a model structure is available. In that situation, model parameters must be estimated before one can proceed with the estimation of the state. The Kalman filter allows the estimation of model parameters through prediction error decomposition (see Harvey [Harvey, 1989]). For a state space parameter estimation method, please see AppendixB.

The Kalman filter provides a minimum mean squared error (MMSE) estimate $\hat{x}_{t}$ of the state $x_{t}$ based on measurements of the environment. Using these estimates of the state it is also possible to formulate predictions of $z_{t}$ by simply substituting the estimate $\hat{x}_{t}^{-}$into the measurement equation in order to obtain a prediction $\hat{z}_{t}^{-}$of $z_{t}$ as follows:

$$
\hat{z}_{t}^{-}=H_{t} \hat{x}_{t}^{-} .
$$

If the model is linear, and if the initial state and model disturbances are distributed normally, the predictions $\hat{z}_{t}^{-}$of $z_{t}$ will also be Gaussian with mean:

$$
\begin{aligned}
\mathbb{E}\left(z_{t}\right) & =\mathbb{E}\left[H_{t} x_{t}+v_{t}\right] \\
& =H_{t} \mathbb{E}\left[x_{t}\right] \\
& =H_{t} \hat{x}_{t}^{-},
\end{aligned}
$$

and with variance:

$$
\begin{aligned}
\operatorname{Var}\left(z_{t}\right) & =\mathbb{E}\left[z_{t}-\mathbb{E}\left(z_{t}\right)\right]\left[z_{t}-\mathbb{E}\left(z_{t}\right)\right]^{T} \\
& =\mathbb{E}\left[H_{t} x_{t}+v_{t}-H_{t} \hat{x}_{t}^{-}\right]\left[H_{t} x_{t}+v_{t}-H_{t} \hat{x}_{t}^{-}\right]^{T} \\
& =\mathbb{E}\left[H_{t}\left(x_{t}-\hat{x}_{t}^{-}\right)+v_{t}\right]\left[H_{t}\left(x_{t}-\hat{x}_{t}^{-}\right)+v_{t}\right]^{T} \\
& =\mathbb{E}\left[H_{t}\left(x_{t}-\hat{x}_{t}^{-}\right)\left(x_{t}-\hat{x}_{\hat{t}}^{-}\right)^{T} H_{t}^{T}+v_{t} v_{t}^{T}\right] \\
& =H_{t} P_{t}^{-} H_{t}^{T}+R_{t} .
\end{aligned}
$$




\subsubsection{Prediction: Univariate Model}

The univariate model of returns attempts to predict stock returns for individual firms based on historical return information. The general form of the univariate model of returns is:

$$
r_{i, t}=r_{i, t}^{\text {fund }}+r_{i, t}^{\text {nfund }}+v_{i, t}
$$

where, $r_{i, t}^{\text {fund }}$ and $r_{i, t}^{\text {nfund }}$ represent respectively the fundamental and non-fundamental part of returns and $i$ is an index corresponding to the stock in question. The model has been introduced in section 4.3 and is described in full detail in Appendix C.

For each of the 10 stocks that have been selected, a separate model is estimated. The model parameters are estimated on 7 years of data (1-Jan-1990 to 31-Dec-1996, a total of 1770 daily retums). Following estimation of the model parameters, a sequence of one-day-ahead predictions are calculated for the remainder of the data (1-Jan-1997 to 3-Jan-2000; 757 daily returns).

Since full information on the initial state vector is not readily available, the initial state vector is initialized as a diffuse prior. The variance is set to $P_{0}=\kappa I$, where $I$ is the identity matrix of appropriate size, and $\kappa$ is a large number. For convenience, it will be assumed that $\kappa=1000$ (see Harvey [Harvey, 1989] p. 121).

The univariate model contains a large number of unknown parameters to be estimated: the univariate model's parameter space, $\Psi$, is composed of the state space model matrices $H$ and $F$ (see equations C.5 and C.5 in Appendix C), as well as of the noise specification matrices $R$ and $Q$ (eqn. C.5): $\Psi=\{H, F, R, Q\}$. More precisely, $H$, the measurement matrix contains $q$ parameters related to the MA model $\psi_{1}, \psi_{2}, \ldots, \psi_{q}$, while the transition matrix $F$ contains $p$ AR parameters: $\phi_{1}, \phi_{2}, \ldots, \phi_{p}$, and, the parameter $\rho$ (see eqn. C.5). Finally, the measurement error covariance, $R$, is a scalar and the transition error covariance matrix only contains 4 components since the day-of-the-week fluctuation errors have the same variance $\sigma_{\chi}^{2}$. Thus the components of matrix $Q$ are: $\sigma_{\eta^{(1)}}^{2}, \sigma_{\eta^{(2)}}^{2}, \sigma_{\chi}^{2}$ and $\sigma_{\omega}^{2}$. In total, the parameter vector for the univariate model has $p+q+6$ components as follows:

$$
\Psi=[H, F, R, Q]=\left[\psi_{1}, \psi_{2}, \ldots, \psi_{q} ; \phi_{1}, \phi_{2}, \ldots, \phi_{p}, \rho ; \sigma_{\nu} ; \sigma_{\eta^{(1)}}^{2}, \sigma_{\eta^{(2)}}^{2}, \sigma_{\chi}^{2}, \sigma_{\omega}^{2}\right]
$$

The original univariate model does not specify the form of the sub-model for the non-fundamental part of returns, and, it has only been assumed that these returns follow the ARMA specification. In order to specify the model fully, the Akaike criterion is used as a guide. The Akaike information criterion (see 
[Harvey, 1989], p. 80) is a goodness of fit measure striving to balance model fit with model parsimony:

$$
A I C=-2 \log L(\Psi)+2 n,
$$

where $\log L(\Psi)$ and $n$ are respectively, model likelihood, and the number of parameters present in the model. Models are selected by minimizing the AIC as a function of the number of parameters. The search for the appropriate model will be conducted on a limited size model-space, composed of 26 models, from $\operatorname{ARMA}(1,0)$ to $\operatorname{ARMA}(5,5)$ in order to avoid over-fitting as much as possible. The best model according to the AIC criterion will be used in the forecasting stage.

\subsubsection{Prediction: Multivariate Model}

The multivariate model proposes to predict stock returns by explaining both return variation due to firm specific effects and variation due to the general market forces. The multivariate model consists of two independent components: one models the behavior of the broad markets, while the other one models the behavior of individual firms. The model has the following form:

$$
r_{i, t}=r_{i, t}^{\text {unsys }}+r_{t}^{\text {sys }}=r_{i, t}^{\text {fund }}+r_{i, t}^{\text {nfund }}+\beta^{\mathrm{Mkt}} \hat{Z}_{t}^{\mathrm{Mkt}(-)}+v_{i, t},
$$

where $r_{i, t}^{\text {unsys }}$ and $r_{t}^{\text {sys }}$ represent respectively the unsystematic and the systematic components of individual stock returns.

The market sub-model of the multivariate model of returns contains 18 parameters. The system transition matrix $F^{\mathrm{Mkt}}$ contains 9 parameters and the system dynamics error covariance matrix $Q^{\mathrm{Mkt}}$ is assumed to be diagonal with 9 parameters. The measurement matrix $H^{\mathrm{Mkt}}$ relating the estimate of the state of the market to measurements is an identity matrix and contains no parameters. The measurement error covariance matrix, $R^{\mathrm{Mkt}}$, is assumed to be fixed as well.

The market sub-model is initialized with diffuse priors. The initial state variance is set to $P_{0}=\kappa I$, where $I$ is the identity matrix of appropriate size, and $\kappa$ is a large number. For convenience, it will be assumed that $\kappa=1000$ (see Harvey [Harvey, 1989] p 121).

Parameter estimation is accomplished through the prediction decomposition method described in Appendix B. The market sub-model is estimated first. It is estimated on the training data spanning the same period as for the univariate model. Using predictions of the market sub-model as well as measurements of the state of the firm (returns), the multivariate model is then estimated. 


\subsubsection{Prediction Windows}

In order to maximize the predictive ability of the proposed models, the predictions will consist of one day ahead forecasts for both the univariate and the multivariate models. The forecast following receipt of Friday's closing prices covers the period of time from Friday close to Monday close.

\subsubsection{Model Testing}

The study has proposed two predictive models for the behaviour of stock returns. The first model utilizes only information concerning the past history of returns in order to estimate their future behaviour. The second model augments the first by including the effects of the broad economy in estimating the state of the firm.

It is important to test the predictive ability of these models as compared to other, simpler models which are already used in the literature. The predictive ability of the models will be compared to the following baseline models:

a random walk,

- AR(1) model,

- an $\operatorname{ARMA}(\mathrm{p}, \mathrm{q})$ model with $\mathrm{GARCH}(1,1)$ Gaussian heteroscedastic errors,

- an ARMA(p,q) model with asymmetric GJR-ARCH Gaussian heteroscedastic errors,

The goal of these baseline models is to find the strengths and weaknesses of the forecasting models proposed. The random walk model will be used to test whether the models estimated in the study can beat the simplest forecasting model: the martingale. The remaining tests consist of comparing the models' performance to an AR(1) specification for returns, as well as some models from the ARCH family of heteroscedastic models.

The predictive ability (for the main models as well as the baseline comparison models) will be examined from three different angles. First the models' general predictive performance will be examined using Theil's inequality coefficient. Second, Pesaran and Timmerman's sign test [Pesaran and Timmerman, 1992] will be used to verify the models' ability to predict the direction of movement in stock prices, and finally, a financial profitability test will examine the money making ability of trading rules based on the various models. 
Theil's inequality coefficient [Theil, 1966] is a measure of a model's predictive ability - it measures the degree of a model's predictive ability over a nochange prediction. Its major advantage compared to other techniques which only report the model's overall fit is to yield insights into the nature of prediction errors, specifically, the average model fit, the variability of predictions, and the concurrence of predictions with the actual values. The statistic has the following form:

$$
U^{2}=\frac{\sum_{t}\left(P_{t}-A_{t}\right)^{2}}{\sum_{t} A_{t}^{2}}
$$

where $P_{t}$ represents predicted change and $A_{t}$ realized change in the phenomena under investigation. A value of $U^{2}=0$ indicates a perfect prediction, while a value $U^{2}=1$ indicates that the model behaves like a no-change rule. The statistic is not bounded and can be greater than 1 if the prediction rule behaves worse than a no-change rule. The statistic can be decomposed into three "proportions":

$$
U^{M}=\frac{(\bar{P}-\bar{A})^{2}}{\frac{1}{n} \sum_{t}\left(P_{t}-A_{t}\right)^{2}}, \quad U^{S}=\frac{\left(s_{P}-s_{A}\right)^{2}}{\frac{1}{n} \sum_{t}\left(P_{t}-A_{t}\right)^{2}}, \quad U^{C}=\frac{2(1-\rho) s_{P} s_{A}}{\frac{1}{n} \sum_{t}\left(P_{t}-A_{t}\right)^{2}},
$$

where, $U^{M}, U^{S}$ and $U^{C}$ are respectively the bias proportion, indicating the average fit of predictions, the variance proportion contrasting the equality of the variances of the observed data and predictions, and the covariance proportion examining the correlation of predictions and realizations. The three statistics sum to one: $U^{M}+U^{S}+U^{C}=1$.

Direction testing consists of checking whether or not a model has forecast the correct direction of change in stock prices: up or down. This type of test is important in verifying the quality of a model for it tests the model's usability with respect to speculation. The test used in this work is the non-parametric sign test proposed by Pesaran and Timmerman [Pesaran and Timmerman, 1992]. Let $Y_{t}=1$, if the realized return, $r_{t}$, is positive (and 0 otherwise), and let $X_{t}=1$ if the predicted retum, $\hat{r}_{t}$, is positive (and 0 otherwise); also let $Z_{t}=1$ if the model correctly predicted the change in price at time $t$, and let $Z_{t}=0$ otherwise. Then, the fraction of times that the model has correctly predicted the change in the price of a stock is $\hat{P}=\frac{1}{T} \sum_{t=1}^{T} Z_{t}$. Assuming independence of $r_{t}$ and $\hat{r}_{t}$, let $\hat{P}_{*}=\hat{P}_{r_{t}} \hat{P}_{\hat{r}_{t}}+\left(1-\hat{P}_{r_{t}}\right)\left(1-\hat{P}_{\hat{r}_{t}}\right)$ be the theoretical fraction of times that the model should predict correctly the changes in price, where $\hat{P}_{r_{t}}=\frac{1}{T} \sum_{t=1}^{T} Y_{t}$ and $\hat{P}_{\hat{r}_{t}}=\frac{1}{T} \sum_{t=1}^{T} X_{t}$. Then, the non-parametric test statistic proposed by Pesaran 
and Timmerman,

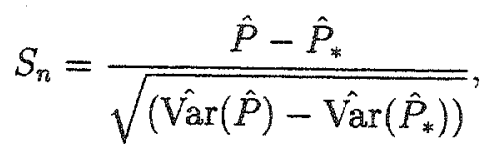

is asymptotically distributed as $N(0,1)$, where, $\hat{\operatorname{Var}}(\hat{P})=\frac{1}{T} \hat{P}_{*}\left(1-\hat{P}_{*}\right)$ and $\hat{\operatorname{Var}}\left(\hat{P}_{*}\right)=\frac{1}{T}\left(2 \hat{P}_{\hat{r}_{t}}-1\right)^{2} \hat{P}_{r_{t}}\left(1-\hat{P}_{r_{t}}\right)+\frac{1}{T}\left(2 \hat{P}_{r_{t}}-1\right)^{2} \hat{P}_{\hat{r}_{t}}\left(1-\hat{P}_{\hat{r}_{t}}\right)$. The test will be conducted on predictions stemming from the univariate and multivariate models, for every stock in the sample, and will be compared to results from the benchmark models.

Prediction model profitability will be tested with the help of simple trading rules. At the beginning of the test period, two investors invest $100 \$$ each into every stock in the data sample and trade according to the two prediction models developed in the study: the first one invests according to the univariate model, and the second according to the multivariate model. At the end of each trading day, a prediction for the following day is computed by the models. If a prediction calls for a rise in the price of a stock, the investor holds onto the stock in question, and if the prediction calls for a fall in the price, the investor sells the stock short. The same process will be repeated for weekly predictions. These operations are repeated for the length of the data sample. Let $G_{i}^{*}$ denote the profits realized on stock $i$ using the multivariate or univariate model ('*'), then,

$$
G_{i}^{*}=100 \cdot \prod_{t=1}^{T}\left[Z_{t}\left(1+\left|r_{i, t}\right|\right)+\left(1-Z_{t}\right)\left(1-\left|r_{i, t}\right|\right)\right],
$$

where $Z_{t}$ is defined as above. The performance of the trading rule just described will be contrasted to the performance of the buy-and-hold strategy for the univariate and multivariate models as well as the baseline benchmarks.

The testing procedures have two goals. The first goal is to assess the predictability of stock returns. The Theil statistic as well as Pesaran and Timmerman's sign test will verify whether the models proposed in the study have any returns forecasting ability over and above the proposed benchmark models. The first of these tests will assess the accuracy of predictions, while the second will assess the ability of the various models to predict falls and rises in stock market prices. The second goal is to test for market efficiency. In order for a market to be efficient, it is necessary that historical information be useless in helping one earn abnormal excess returns. Testing the profitability of the predictive models will help answer this question. 


\section{Chapter 5}

\section{Results}

\subsection{Introduction}

The study presents a new approach to the prediction of the behaviour of stock returns by attempting to emulate the analytical behaviour of investors. It proposes that investors strive to make investment decisions by estimating the condition of the markets and the economy, as well as the condition of firms traded on financial markets. It further states that it is based on these perceptions of the states of the various investment targets, that investors make the ultimate investment decisions.

The study suggests that this behaviour can be compared to the the process of filtering widely known in statistical literature. In this statistical framework, incoming information is "filtered" by weighing it according to its inherent accuracy in order to extract from it an unknown and unobservable (directly) quantity of interest. It is believed that this process mimics the behaviour of investors and proposes it as a solution to the problem of making investment decisions.

The study has presented a univariate and a multivariate model for predicting the behaviour of stock returns. The univariate model attempts to forecast the behaviour of a firm's stock returns by looking exclusively at it's historical returns record, believing that this record contains all information necessary to estimate the state of the firm. The multivariate model, on the other hand, additionally utilizes information about the condition of the economy in order to help to predict the behaviour of individual stock returns.

This chapter presents results obtained in the study. The study examined the predictability of stock returns using a state space representation and the Kalman filter. The framework proposed that investors priced stock by attempting to es- 
tablish its intrinsic value or "real condition" based on available information, in effect weighting new and old, and reliable and unreliable information in a manner similar to that of the Kalman filter. The study thus hypothesized that investors' behaviour could be approximated through the use of engineering filtering techniques. The hypothesis was tested by attempting to predict the behaviour of stock returns using this new framework.

The chapter is divided as follows. First, the data and parameter estimation algorithms are presented. Further, estimation results are presented for the univariate and multivariate models. Finally, prediction results are discussed.

\subsection{The Data}

The data consisted of stock returns for 10 randomly selected stocks belonging to the Dow Jones Industrial Average as of January 2000. The data spans the period from 1, January 1990 to 1, January 2000. Financial data consisted of returns on the DJ Composite, Nikkei 225 and FTSE 100 stock indices, the short, long, and risky bond rates, as well as the exchange rates for the UK and Japan. Further information about the data is presented in the the Data Appendix A.

The data was divided into two periods. The estimation period spans from January 1,1990 to December 31, 1996, and consists of 1770 daily return observations, while the model testing period, spans the remainder of the data, (January 1, 1997 to January 1,2000 ) and contains around 760 observations. The models are first estimated on the first segment of the data, and their prediction capability is tested on the second segment. Table 5.1 presents a summary of the behaviour of the stock prices for the testing period.

\subsection{The Estimation Method}

A major challenge consisted in developing a general estimator for state space models to be used in the study. In particular, the following problem had to be solved - given the general linear state space system,

$$
\begin{aligned}
z\left(t_{i}\right) & =H\left(t_{i}\right) x\left(t_{i}\right)+B_{2}\left(t_{i}\right) u_{2}\left(t_{i}\right)+v\left(t_{i}\right), \\
x\left(t_{i+1}\right) & =F\left(t_{i}\right) x\left(t_{i}\right)+B_{1}\left(t_{i}\right) u_{1}\left(t_{i}\right)+G\left(t_{i}\right) w\left(t_{i}\right),
\end{aligned}
$$

estimate the state of the system $x$, and, any unknown parameters in the system matrices $F(\cdot), H(\cdot), B_{1}(\cdot), B_{2}(\cdot), Q(\cdot)$ and $R(\cdot)$ given system measurements $z$. 


\begin{tabular}{|l|r|r|r|}
\hline \hline Ticker & 2-Jan-97 & 31-Dec-99 & \% Change \\
\hline \hline BA & 51.08 & 41.14 & -20 \\
C & 19.44 & 55.39 & 184 \\
GE & 15.53 & 51.43 & 231 \\
IBM & 37.98 & 107.63 & 183 \\
INTC & 32.5 & 82.26 & 153 \\
JPM & 86.3 & 124.49 & 44 \\
MMM & 79.55 & 97.24 & 22 \\
MRK & 37.68 & 66.61 & 76 \\
MSFT & 20.4 & 116.75 & 472 \\
XOM & 48.11 & 79.68 & 65 \\
\hline B\&H Strategy & 1000 & 2410 & 141 \\
\hline DJComp. & 2015 & 3214 & 59 \\
SP500 & 737 & 1469 & 99 \\
\hline \hline
\end{tabular}

Table 5.1: Dividend and split adjusted stock prices and returns (in percent), on a buy-and-hold strategy holding individual stocks for the period January 11997 - December 31 1999. The return from an equally weighted buy-and-hold strategy based on a $100 \$$ investment in each of the 10 stocks is also presented. For comparison purposes, returns on the DJ Composite and SP500 indices are also presented. 
The solution was accomplished following the work of Maybeck [Maybeck, 1979, Maybeck, 1981, Maybeck, 1972].

In his work, Maybeck approached the parameter estimation problem using the method of maximum likelihood. He proposed that the estimates of parameters belonging to system matrices were most efficiently handled through the use of iterative maximum likelihood methods, and in particular Rao's scoring. This approach combines Kalman filter recursions in parallel with recursions needed for maximum likelihood computations. This procedure can be used for estimation and model fitting on the full dataset. It is also conveniently structured for time varying parameter estimation based on a moving sub-sample of data. This latter technique has been developed to remedy some of the observed deficiencies of the proposed model and will be described and discussed later in the thesis.

The estimation algorithm is fully presented in Appendix B. The algorithm was implemented in the SciLab programming language [Cergrene, 2001], a computing environment similar to MatLab, available freely at http://www-rocq.inria.fr/scilab/. The state space representations of the models proposed in the study are presented in Appendix $\mathrm{C}$ and section 4.4. The computation required for the $\mathrm{ARCH}$ models was performed with OX version 3.0 of Doornik [Doornik, 1999] and the G@RCH toolbox (version 2.2) of Laurent and Peters [Laurent and Peters, 2002].

In the light of Brooks, Burke and Persand [Brooks et al., 2001], it is known that different ARCH software packages tend to yield differing parameter estimates and test statistics. Laurent and Peters [Laurent and Peters, 2002], tested the reliability of OX and $\mathrm{G} @ \mathrm{RCH}$ against several other econometric softwares, using the same methodology and datasets as Brooks, Burke and Persand. The results show that $\mathrm{G} @ \mathrm{RCH}$ performs better than commonly available software, yielding smaller estimate errors. This evidence suggests that $\mathrm{OX}$ and $\mathrm{G} @ \mathrm{RCH}$ are a reliable tool in estimating heteroscedastic models.

\subsection{Estimation Results - Univariate Model}

The univariate model proposed that the state of the firm could be attributed to two distinct influences: the fundamental and non-fundamental components. The fundamental component described the real growth of the firm based on reliable information, while the non-fundamental component described remaining part of returns due to investor psychology. It was hypothesized that the fundamental component of returns followed a damped linear trend model, while the non-fundamental part of returns followed an ARMA process with a daily component. Further de- 
tails on the structure of the univariate model are given in Appendix C.

\subsubsection{Variance Assumptions}

In order to estimate the model, a variance related assumption was made. More precisely, it was assumed that $R$, the variance of the measurement noise $v_{t}$, was known. This assumption was made for purposes of tractability - the reason is that the estimation algorithm is not well behaved when both, system dynamics, and, measurement error variances (respectively, $Q$ and $R$ ), are estimated simultaneously (see Maybeck [Maybeck, 1981], p. 122). This assumption also simplified the computation process. Initially, the variance of the measurement error was set to be relatively small, with $R(\cdot)=0.1$. The choice of this variance was motivated by the consideration that not all of the returns is noise, and that returns, while containing noise, also contain information useful for their prediction. A value of 0.1 , compared to 1 or 0.5 for the measurement noise variance, it is believed, is a reasonable guess as to the level of noise present in the data. A sensitivity analysis, however, was performed to examine model behaviour under different levels of measurement noise ranging from $R(\cdot)=0.01$ to $R(\cdot)=1$. The results of this analysis are presented later in the text.

\subsubsection{Non-fundamental model component: daily effects}

The univariate model contains a sub-model designed to capture the daily effect in stock returns. Indeed, the literature has shown that the day-of-the-week effect is a significant phenomenon in stock returns. The daily component was first modelled using Harvey's time varying daily effects model (see Harvey [Harvey, 1989] p. 173). Appendix C presents the details of this model specification.

The first estimation runs of the full univariate model with daily effects produced signs of model misspecification (negative estimated system noise variances). In order to examine the phenomenon further, only daily effects were fitted to returns data. This produced a relatively bad fit. Table 5.2 presents the system variance parameter $(Q)$ obtained by fitting Harvey's daily effects model to stock returns data. It also presents the out-of-sample predictive ability of this model. Figure 5.1 plots the out-of-sample forecasts from the model for stock BA against the realized returns.

As can be noted from the statistics provided in the table, the fit of the model is very poor. Theil statistics exceed 1 (they are on average 2 ) indicating the model predicts worse than a no-change rule. While the $U^{M}$ and $U^{S}$ statistics state that 
that the model can fit relatively well the mean and variance of the data, $U^{C}$ states that the predictions do not covary with realized returns. The sign test indicates that the model was able to correctly predict the sign of returns only for stocks JPM and XOM (test statistics 2.96 and 2.25 respectively). The trading rule yields profits for 3 stocks: GE (15\%), JPM (73\%) and XOM (65\%) (results in excess of $100 \$$ indicate profits, while those below, indicate losses on following the model based trading rule). The profits for JPM and XOM correspond to the model's ability to accurately predict the sign of returns as indicated by Pesaran and Timmerman's test. On average the trading rule loses money, with $896 \$$ left after starting with $1000 \$$ invested equally in all the stocks. Thus, on itself, Harvey's daily effects model cannot explain or predict the behaviour of stock returns very well.

To further test the problem, a second daily effects model based on dummy variables for the different days of the week (see Harvey [Harvey, 1989] p. 172) was estimated on the data. The results have shown a poor fit as well. Table 5.3 presents the estimated system variance parameter $(Q)$ and the out-of-sample predictive ability of the dummy variable model. Figure 5.2 plots the out-of-sample forecasts from the model for stock BA against the realized returns.

The dummy variable daily effects model presents even poorer results than Harvey's model. Theil statistics are close to 4 on average, and exceed those from Harvey's model. As with Harvey's daily effects model, the $U^{M}$ and $U^{S}$ statistics indicate that the model fits the mean and variance of the data relatively well, but the predictions do not covary with the data. The Pesaran-Timmerman test indicates that signs were predicted successfully for stocks C and JPM, but that predictions for stocks BA, MMM and XOM show almost significant results. The test statistics also seem a little larger than for Harvey's model. The stocks which displayed a profit are BA (36\% return), GE (25\%), JPM (2.7\%), MMM (66\%), and XOM (92\%). The profit for the whole portfolio of 10 stocks is $1092 \$$ on an initial investment of $1000 \$$ which translates into a return of $9 \%$.

A buy-and-hold equally weighted strategy, holding the 10 stocks for the length of the test period yielded the results as presented in Table 5.1. Comparing the results of the daily effects models with the buy-and-hold, clearly indicates that with the exceptions of trading strategies for the stocks BA, JPM, MMM and XOM, holding the individual stocks yields higher profits. This analysis does not include transaction costs necessary to implement the predictions. Interestingly, the dummy variable model yielded a $35 \%$ profit on Boeing, while the shares in this stock fell $20 \%$ in the same period. Holding a portfolio of the 10 stocks for the testing period yielded a return of $74 \%$ (see Table 5.1). In contrast, following the daily effects models yielded returns of $-10.4 \%$ and $9 \%$ for the Harvey and dummy 
variable approaches respectively.

In conclusion, using the day-of-the week effect in order to predict returns yields unfavorable results. The results show that the daily effects models by themselves cannot predict the behaviour of returns, but that they can, to a certain degree, predict the direction of changes in prices. The predictive ability obtained from these models, is, however, poor.

To further examine the problem, two univariate models including non-fundamental elements only, one with the Harvey, the other with the dummy variable daily effects specification, with an AR(1) specification for the psychological effects, were estimated. For details, the reader is referred to Appendix C.

Combining the daily component with the $A R(1)$ effect produced an unexpected result - the variances for the daily effects became negative and small in magnitude, for both the Harvey and dummy variables specification. This suggests that a simple AR(1) model can explain the day-of-the week anomaly. Thus it appears that a time-varying daily effects model can fit returns data on its own. Unfortunately, when used in conjunction with an ARMA model, the day-of-week effect seems to disappear. It is possible therefore that the day-of-the-week effect found in previous studies is simply a statistical artifact due to model misspecification. In conclusion, since the daily component does not seem to contribute explanatory power, it was dropped from the model.

\subsubsection{Fundamental model component: damped linear trend}

In the model, the fundamental component represents the real value of the firm, and was described using a damped linear trend. Refer to Appendix $C$ for further detail.

Fits of a model with the damped linear trend component and the non-fundamental ARMA component, however, have shown model misspecification, with variances in the fundamental component becoming negative. In order to solve the problem, several variations of the damped linear trend model were examined. Unfortunately, the model always showed misspecification.

The fundamental sub-model was therefore eliminated from the main model. After these changes were implemented, the univariate model describes stock returns using the non-fundamental component, or ARMA formulations. 


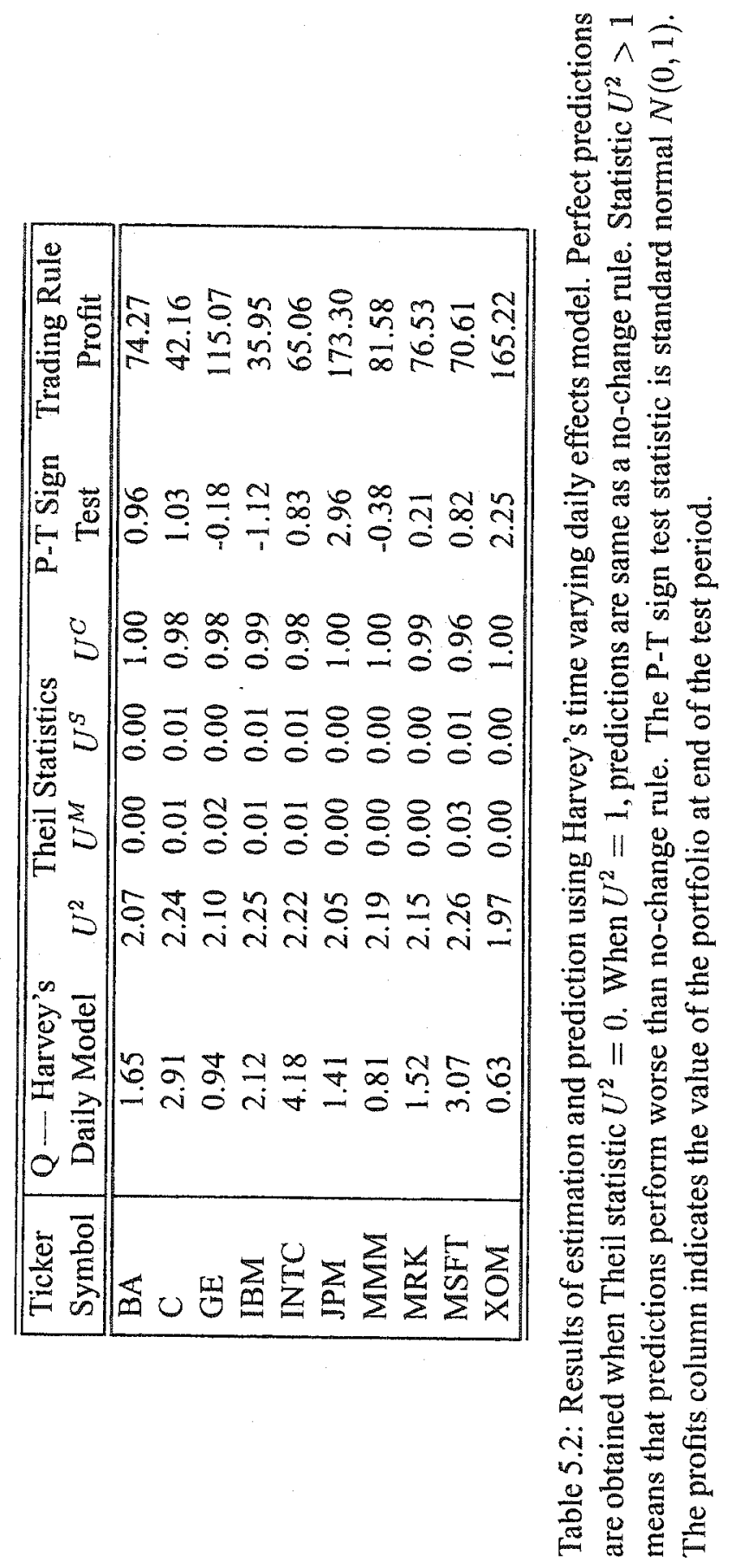




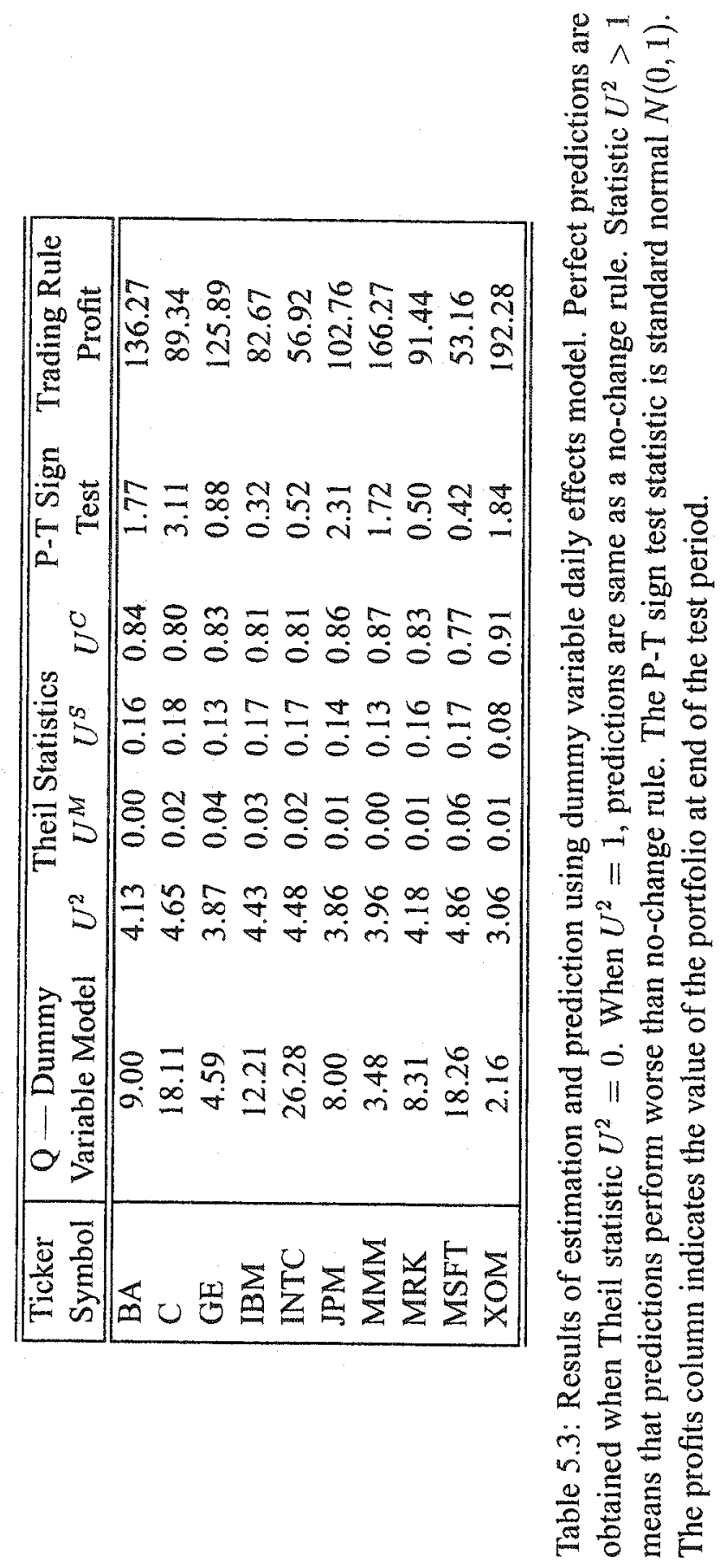




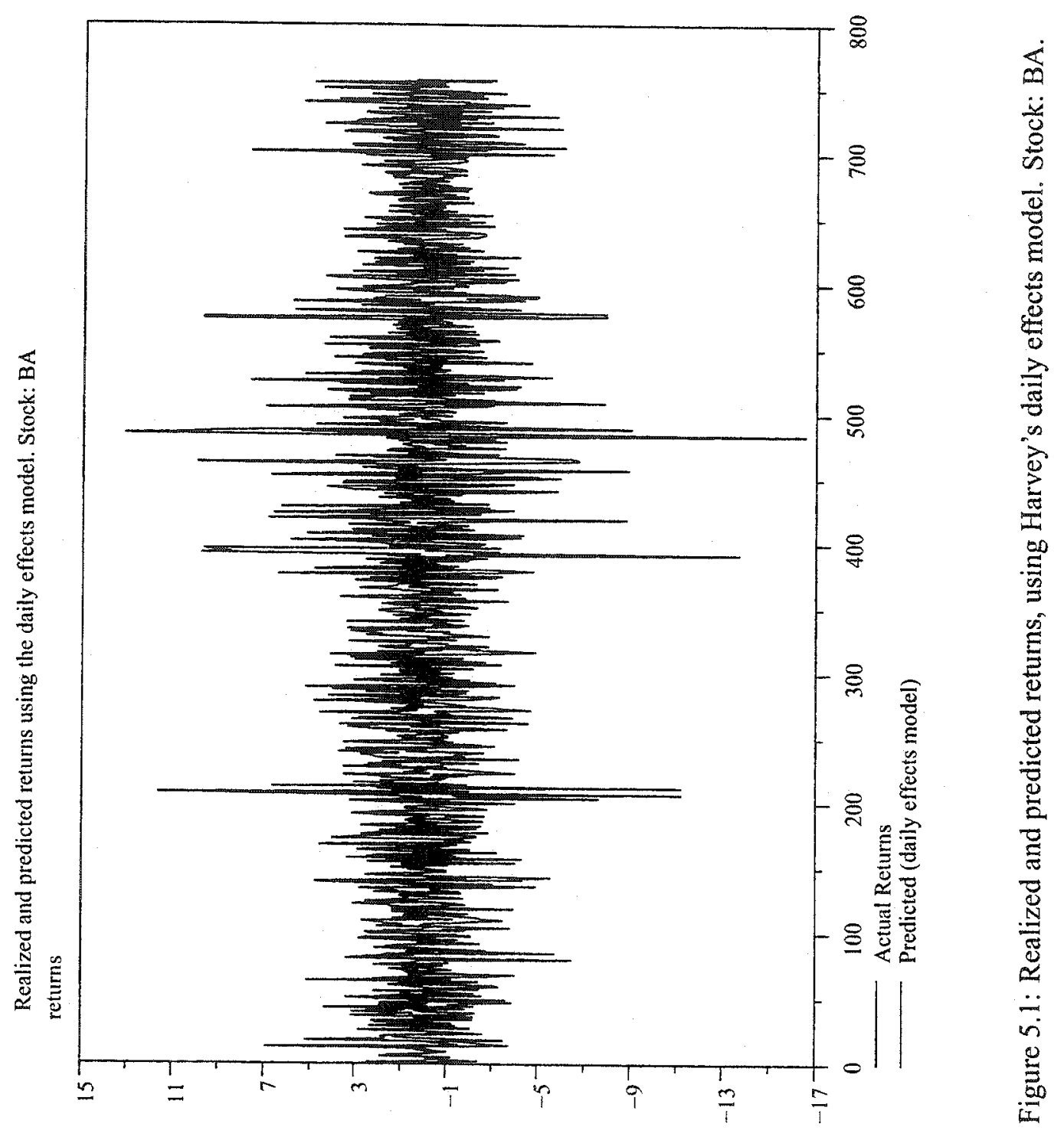




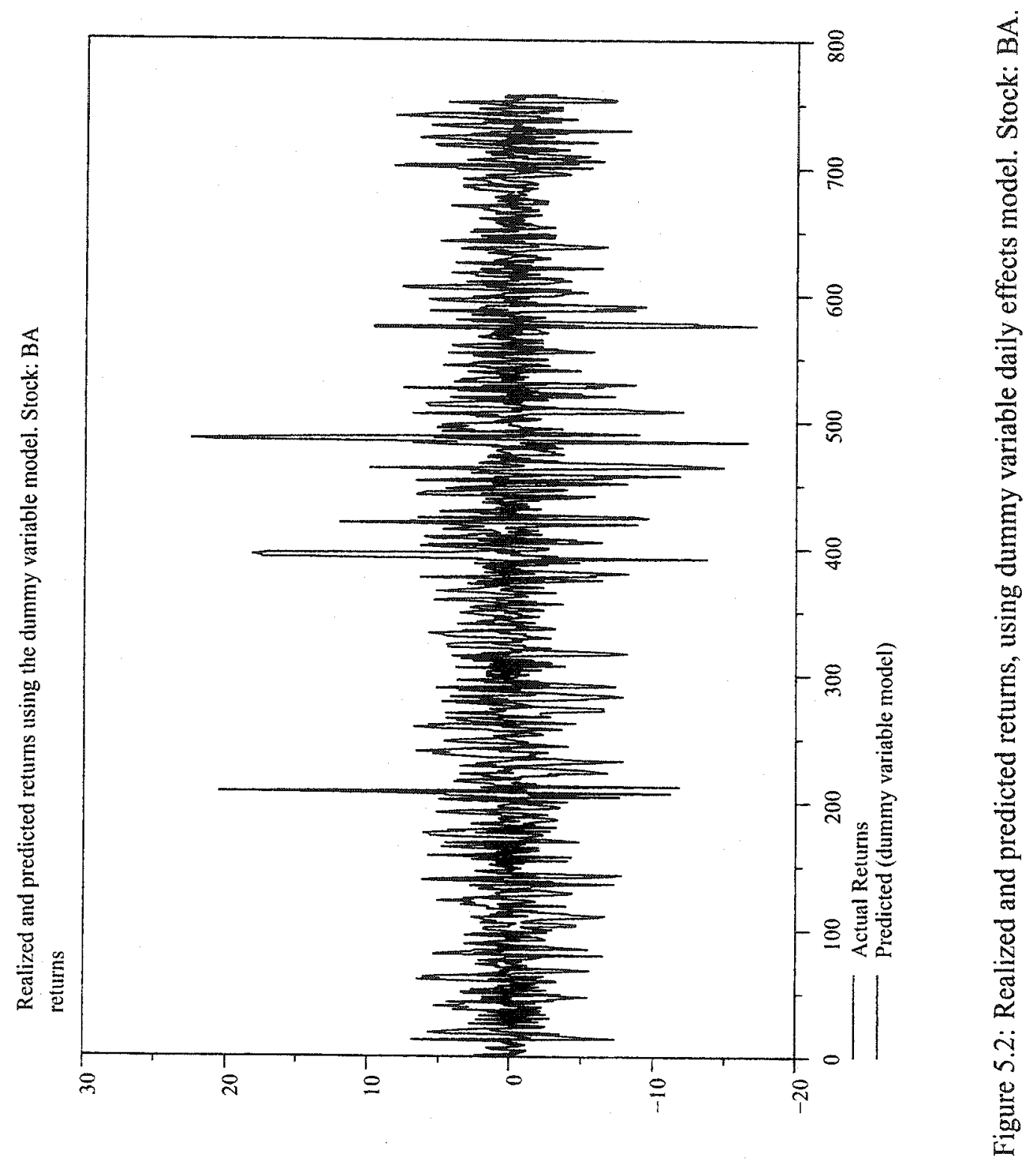




\subsubsection{Non-fundamental model component: ARMA sub-model}

The ARMA sub-model describes the variation in returns due to investor psychology. After identifying problems with the daily and fundamental components, only the ARMA sub-model was left remaining in the univariate model.

For each of the 10 stocks, 26 variations of the univariate model were estimated using 1770 returns spanning from January 11990 to December 31 1996. The univariate models consist of simple ARMA(p,q) formulations for returns, misspecification having reduced the more complex model. For each stock, the models ranged from an $A R(1)$ specification up to $\operatorname{ARMA}(5,5)$. The models were estimated using the algorithm described in Section 5.3.

For each stock, a single best model was selected based on the Akaike criterion. Since many of the different ARMA formulations produced very similar results, it was often difficult to establish the best model on the basis of the AIC criterion. In such cases the models were also selected on the basis of parsimony. The appendix presents the complete summary of parameter estimation results and graphs. Table 5.4, page 111, presents the specifications of the models so selected.

.Tables D.1-D.10 (Appendix D) show the estimated parameters and model diagnostics, that is the likelihood and the AIC criteria for the fitted models. The Appendix further pictures plots of the predicted versus realized stock returns in Figures D.1-D.10. Tables G.1-G.10 (page 209), present a summary of all models considered in this study.

\subsection{Estimation Results - Multivariate Model}

The multivariate model predicts stock returns based on the examination of the systematic and unsystematic components of returns. The multivariate models were based on the univariate models selected in section 5.4.4.

For each of the 10 stocks, the best fitting univariate model representation was selected and augmented to include the market component as an explanatory variable (refer to section 4.4 and section 4.4 .

The models were re-estimated, new parameters obtained, and predictions were performed over out-of-sample data. The estimation results as well as prediction plots are presented in Appendix E. The appendix contains the estimate of the market sub-model in section E.1. The section contains the estimate of the state transition matrix $F$ and the system noise covariance matrix $Q$.

Parameter estimates for the full multivariate model are presented in section E.2. 
Prediction results for the multivariate model are displayed in Table 5.5 (p. 113). For comparison purposes, the multivariate models' results are contrasted to those of other models in tables G.1-G.10.

\subsection{Prediction Results: Univariate Model}

From among the 26 models for each of the 10 stocks, a single model was selected to be used in predicting stock returns. The results of that estimation procedure are displayed in the appropriate appendix.

Once estimated, the models were tested on the subsequent segment of data spanning from January 11997 to January 12000 (see the Appendix A). The forecasts consisted of one-day-ahead predictions. The forecasts produced by these best models were then tested for accuracy.

Three tests were performed. Predictive performance was tested using Theil's criterion, a sign test was performed in order to test for the models' ability to predict the sign of changes in stock prices, and, finally, a trading rule tested the moneymaking ability of the models. Table 5.4 presents the results of these tests and constitutes a summary of the predictive ability of the models. The first column of the table identifies the stock, the next two columns display the order of the best model. The remaining columns display the statistics related to the predictive power of the model. Tables G.1-G.10 compare forecasting results from this and other models.

The Theil statistics $U^{2}$ indicate that the models have a difficult time fitting returns data. Theil statistics close to 1 indicate a poor fit. The $U^{S}$ statistic indicates that the models have problems fitting the highly volatile returns data. This is confirmed by the graphics presented in Appendix D, Figures D.1-D.10. The models predict the signs of returns for stocks BA, MRK and XOM which exhibit P-T values of at least 2.4 . Stock $C$ also suggests weak evidence of sign predictability $(\mathrm{P}-\mathrm{T}=1.75)$, while there is little evidence of predictability for the remaining 6 stocks.

The last column of the table presents the value of the portfolio at the end of the testing period. The trading rule reports profits for stocks BA, C, IBM, XOM and a little for $\mathrm{MMM}$. The trading rule returns for these companies are as high $320 \%$ and as low as $5 \%$. The most profitable strategies correspond to models for which the PT-statistic was high (BA, C, MRK, XOM). The profits do not appear to be explainable by the Theil statistics, and, instead seem to be related to the model's ability to predict the change in prices as expressed by the P-T test. Indeed, 
stocks with high P-T statistic values seem to register trading rule profits. The only exception is the stock $\mathrm{C}$, which registers a profit, even though its P-T statistic is small. The trading rule does not account for trading costs, which can vary across market participants.

On an investment of 1000 divided equally among the 10 stocks, the trading rule would yield $1740 \$$, or a yield of $74 \%$. The total profits from following the trading rules for the portfolio of 10 stocks are positive and the profits are concentrated on the few stocks already mentioned. This figure would be significantly reduced if transaction costs were to be included in the profit computations. By contrast, over the same period of time a buy-and-hold strategy based on the DIIA would yield a return of $59 \%$, and an investment in the SP500 would yield a return of $99 \%$. An equally weighted buy-and-hold investment in the 10 stocks would yield a return of $141 \%$, indicating that the model performs worse than a buy-andhold strategy. It is interesting to note, however, that the standard deviation of returns is higher for the buy-and-hold strategy than for the model based trading rule: 18 and 15 respectively.

\subsection{Prediction Results: Multivariate Model}

Tables 5.5, and, G.1-G.10, present the summary of the predictive ability of the model. Appendix E presents tables of parameter estimates.

The Theil statistics indicate that the multivariate model does not predict better than the univariate model. The Theil statistics are very large, and $U^{S}$ indicates that the failure to track the variance of returns is the biggest culprit in the bad predictive fit of the model. It is important to note however that the $U^{S}$ statistic is slightly smaller for the multivariate model, indicating that market variables can explain some of the variance of returns. The $U^{C}$ statistic also indicates that the correlation between the predictions and observations is small. The graphics in Appendix E, Figures E.1-E.10, show that the variance of returns is not captured well by the model.

The Pesaran and Timmerman sign test suggests that the model could predict the signs of returns for MMM and XOM and seems to be able to predict can predict them for BA, C, and JPM. This performance is similar to that of the univariate model, except that in the former case the statistics were slightly more significant.

Table 5.5 shows that five out of the 10 stocks show a trading rule gain for the testing period. These stocks are C, IBM, INTC, MMM and XOM. These stocks correspond roughly to the stocks for which the sign of returns was predicted well, 


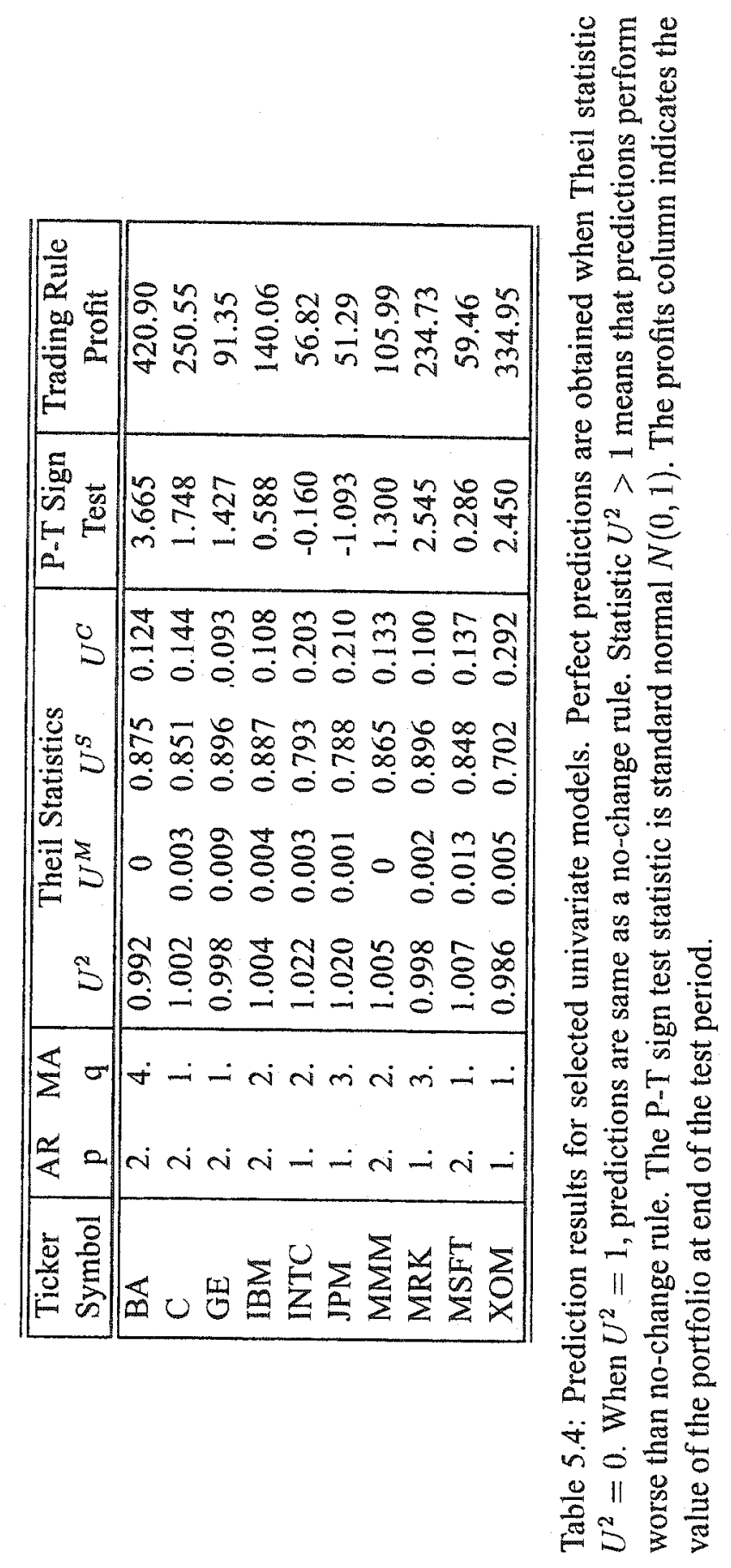


with the exception of IBM for whom the P-T statistic is rather low.

When it comes to the trading rule performance of the multivariate model, an original $1000 \$$ investment into the stocks at the beginning of 1997 , would result in $1758 \$$ in January 2000 , for a combined return of $75 \%$. Again, it is important to stress that this number does not include transaction costs which could have a significant impact on the profitability of the strategy. This figure is only a marginal increase over the profits obtained from following a strategy based on the univariate model. The analysis seems to indicate that the increase in predictive performance due to the inclusion of the market and economic factors is small.

The trading rules based on the univariate model outperform buy-and-hold strategies for four out of the 10 stocks considered in the study, that is BA, C, MRK and XOM. Forecasts for IBM and MMM also create positive returns, however, they do not outperform the buy-and-hold model. It is these stock forecasts that drive the returns obtained from following the active trading strategy. For the remaining stocks (GE, INTC, JPM and MSFT), the models lose money, with the worst forecasts being performed for JPM, the trading rule denoting a loss of $50 \%$, while in reality the stock gained $44 \%$ over the period considered. All these latter stocks recorded significant gains over the test period, gains which the models and trading rules were not able to exploit. In all, out of the 10 stocks, only 4 yield negative returns over the testing period, while the remaining 6 make money, and, only 4 out of these, however, manage to outperform the buy-and-hold strategy. This explains the model under-performing the index based buy-and-hold strategies.

The analysis of the profitability of the multivariate models is similar to that of the univariate models. Four stocks out of 10 drive the profits from active trading rules: C, INTC, MMM and XOM. It is interesting to note that both univariate and multivariate models for $\mathrm{C}$ and XOM manage to beat the buy-and-hold strategy for these stocks. Contrasted with the univariate model, the stocks BA and MRK, are no longer well predicted using the multivariate model, while INTC and MMM are, but were not under the univariate model. Again, active strategies for two stocks (IBM and MRK), while not losing money, did not outperform the buy-andhold models, and the four remaining stock lost money based on the active trading rule. It is an open question as to why are some stocks "easier" to forecasts than others, and in particular $\mathrm{C}$ and XOM. One possibility is that this phenomenon is sample-specific, and the other that these stocks have behaviors which make them inherently easier to predict.

The models a-priori outperform the buy-and-hold strategy based on the DJ Composite but under-perform that based on the SP500: a buy-and-hold strategy 


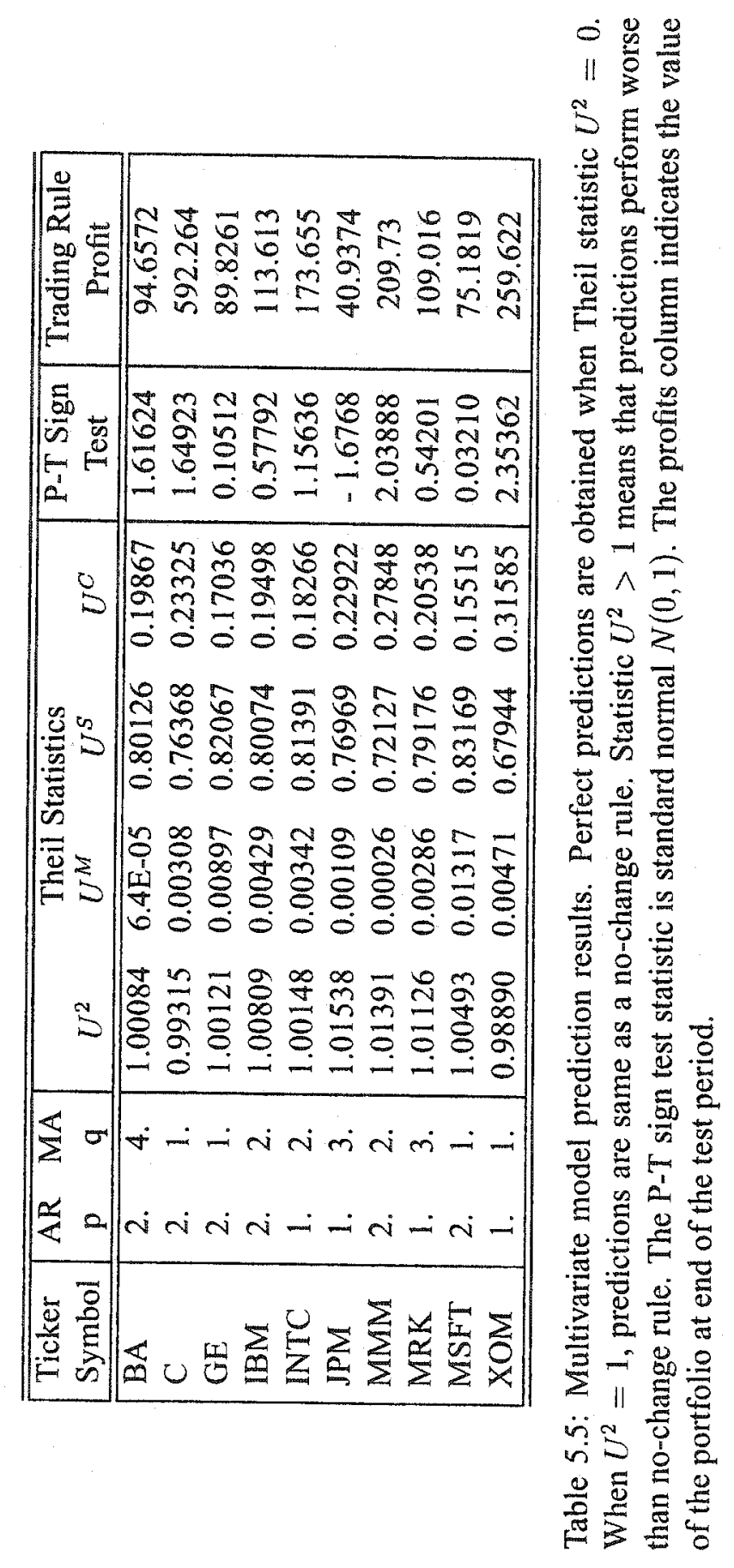


consisting of buying the DJ Composite index on January 21997 and selling it on December 311999 would comparatively yield a return of $59 \%$, and an investment in the SP500 would yield a return of $99 \%$ over the same period. Pursuing a buyand-hold strategy based on buying a portfolio consisting of the 10 stocks, from January 21997 to December 311999 , would yield a total return of $141 \%$. This demonstrates the buy-and-hold strategy to be superior to the model based trading rules.

\subsubsection{Benchmark Model Comparisons}

The performance of the models studied herein was contrasted to the performance of several benchmark models: a random walk model, an AR(1) autoregressive specification and two heteroscedastic model specifications GARCH and GJRARCH. In order to help in the task of comparing the proposed models' performance with that of the benchmark models, complete summary tables are presented in Appendix G, Tables G.1-G.10, page 209.

\subsubsection{Simple time series benchmark models}

Random walk model predictions consist of lagged values of realized returns. Table 5.6 presents the summary of the predictive ability of the random walk model. The Theil criterion indicates that the predictive fit of the models is bad. Interestingly, the $U^{S}$ statistic is very low: this indicates that lagged returns can explain to a certain extent the heteroscedasticity of returns.

When it comes to the Pesaran-Timmerman sign test, the random walk model could correctly predict the sign of retums for only three companies, BA, GE and JPM. The trading rule test shows that the performance of the random walk model is positive for roughly half of the sample of companies. The total profit for the period, based on an initial investment of $1000 \$$, is $426 \$$, for a return of $42 \%$. This figure is lower that that for the active strategy based on the univariate model. This indicates that while a model-based active strategy cannot outperform a buyand-hold investment strategy, the model based strategy performs better than the simple random walk model. It is unclear whether factoring in transaction costs would drive active strategy retums below those of the random walk model.

The AR(1) model performed poorly as well. The Theil statistic indicates the same symptoms as for the other models: the poor fit is caused mainly by the model's inability to capture the heteroscedastic nature of stock returns. The Pesaran-Timmerman sign test indicates that the AR(1) model could predict the 

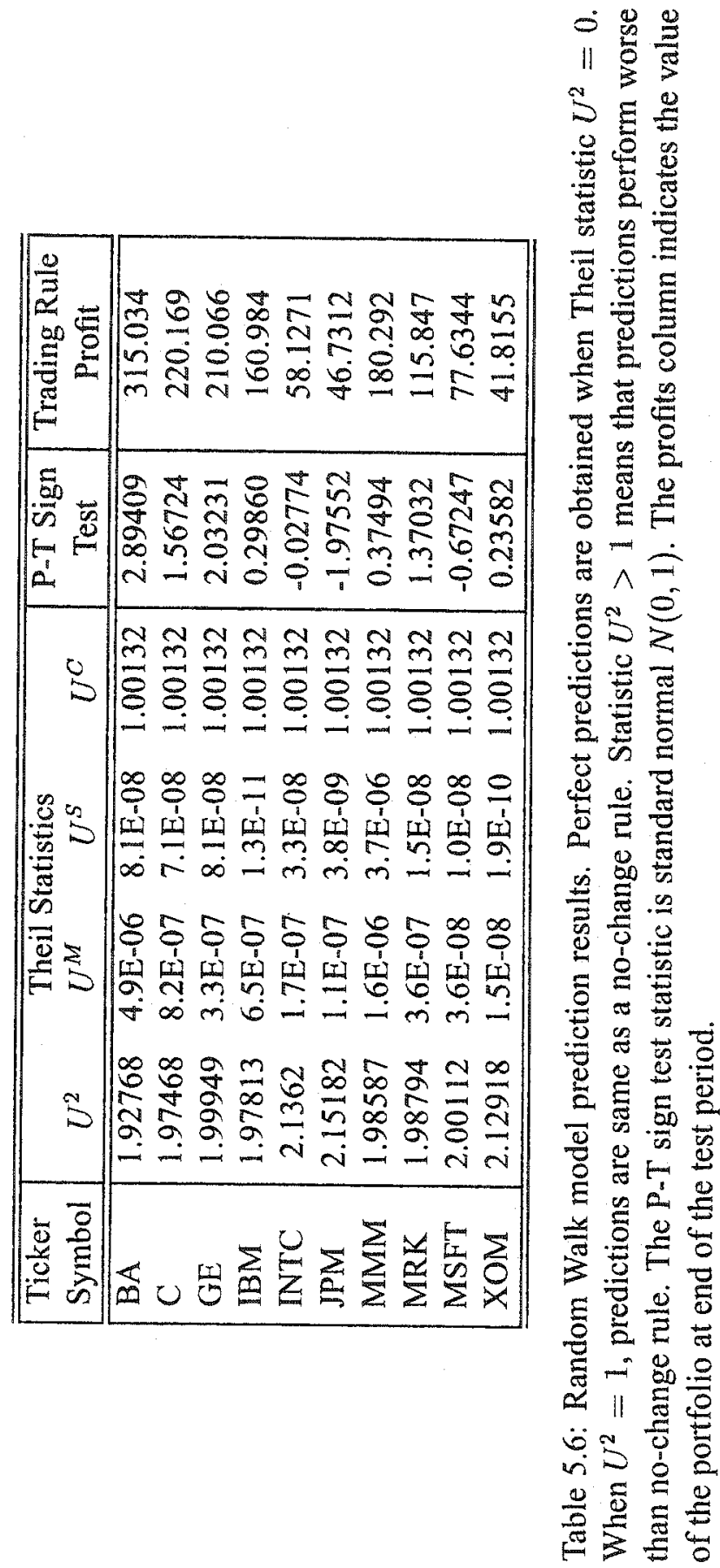
sign of returns only for BA, GE, JPM and XOM. The trading rule indicates an overall profit of $262 \$$ for an investment of 1000 , for a return of $26 \%$.

\subsubsection{GARCH model benchmarks}

An ARCH model allows for the modelling of the variance of the residuals in a time series, and has, therefore, great utility in finance, where highly volatile time series are often encountered. In a GARCH model, the residuals' conditional variance follows an ARMA process, and positive and negative returns affect the variance in the same manner. Full details about the GARCH model are presented in Bollerslev [Bollerslev, 1986]. The GJR-GARCH model, proposed by Glosten, Jagannathan and Runkle [Glosten et al., 1993], extends the GARCH model by allowing negative and positive returns to affect the conditional variance of returns in an asymmetric manner. Table 5.8 examines prediction performance obtained from the two ARCH-type models (a GARCH(1,1) model and a GJR-ARCH model), while Figures E.11-E.20 present the returns forecasts plots.

The models were estimated using the same specification for the mean as the selected univariate models (an ARMA(p,q) model). The goal of the test was to verify whether allowing a model to fit the heteroscedastic nature of returns better, would yield better retum predictions. It is important to note from the start that the GJR model for stock GE did not converge and that results are not available for that stock.

The results show a small improvement over the univariate models. The Theil statistics show the same story as for the non-heteroscedastic models: using the ARMA specification for the mean, the models are not able to fit the data in any reasonable fashion. The biggest problem concerns the $U^{S}$ statistic which is as high as for the normal models. In fact the magnitude of this statistic has not changed much from that from the original models - the lack of the ability to explain the variance of retums is further confirmed by the graphs. It can be also noticed that the P-T statistic even shows a decrease in the accuracy of predictions using the heteroscedastic models. This is confirmed by trading rule results. An investment in the GARCH model strategy would yield a profit of $50 \%$ over the period considered, while an investment in a GJR based strategy would yield only $20 \%$. This discrepancy between the GARCH and G.JR model specifications seems to indicate that even though it is simpler, the GARCH model can explain the data slightly better. It is also interesting to note, that using the heteroscedastic models, trading rule profits are similar between the two variance specifications for all stocks in the sample. It is important to note however that using these more 


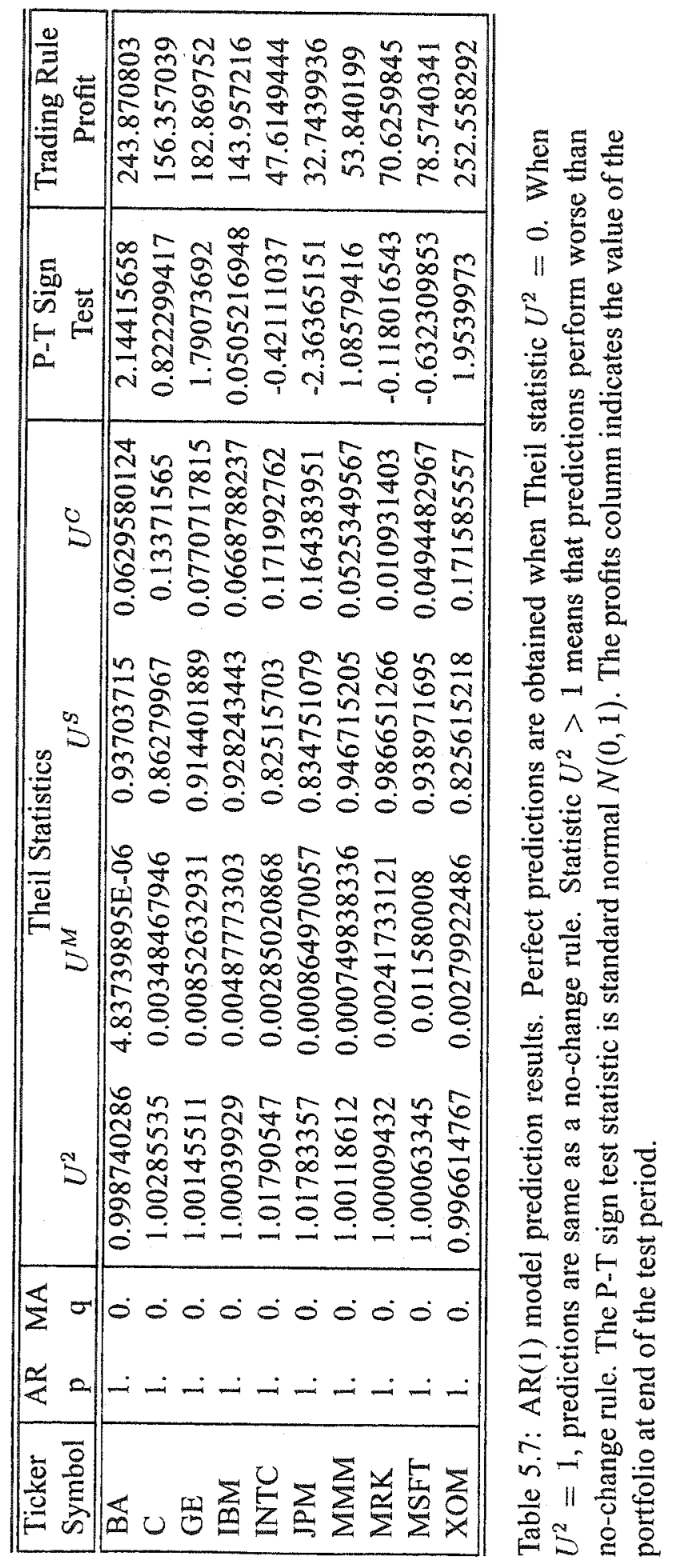


complicated models did not yield better prediction results than using the models proposed in the study.

The comparison of the univariate and multivariate models to the simple baseline models raises several points. First, the predictive fit of all models seems to be inadequate. The Theil statistics indicate that the fault lies in the models' inability to explain the heteroscedasticity present in stock returns. The only exception to this case is the random walk model which is able to explain most of the heteroscedasticity, but unfortunately cannot predict well. The second point concerns the ability to predict the sign of returns: all models are able to do so only for roughly half of the 10 stocks. This ability does not seem to change across models. Thirdly, when it comes to trading rule performance, the multivariate and univariate models seem to have the same predictive ability, while the baseline models perform significantly worse. Indeed the model based returns are roughly $75 \%$ while baseline returns are lower, in the range of $20 \%$ and $40 \%$. Unfortunately, these results are outperformed by simple buy-and-hold strategies - a strategy based on buying the 10 stocks would more than double the money initially invested. The two models proposed in the study seem, however, to be able to outperform the simple baseline models, indicating that returns contain certain information useful to forecasting purposes, at least to a certain degree.

The reason for model under-performance is the fact that the models were only able to outperform the buy-and-hold strategy for 4 out of the 10 stocks for both the univariate and multivariate cases while the remaining forecasts failed to generate yields big enough to cover buy-and-hold returns. It is important to note that the profitability of the active trading rules is highly related to the strength of the Pesaran and Timmerman sign test: stock for which forecasts have a higher value of the PT statistic tend to outperform the buy-and-hold trading rules. For the univariate model this is the case for stocks $B A, M R K$ and XOM, while in the case of the multivariate model this occurs for stocks such as C, MMM and XOM. This confirms that the accuracy in the prediction of changes in prices is the most important factor in a profitable trading rule. The summary of all models discussed in this chapter is presented in Tables G.1-G.10, starting on page 209.

\subsection{Sensitivity analysis}

This section presents the results of a sensitivity analysis performed on the univariate model of returns. In the original model of returns, an assumption was made concerning the level of noise in the measurements. The model assumed that 


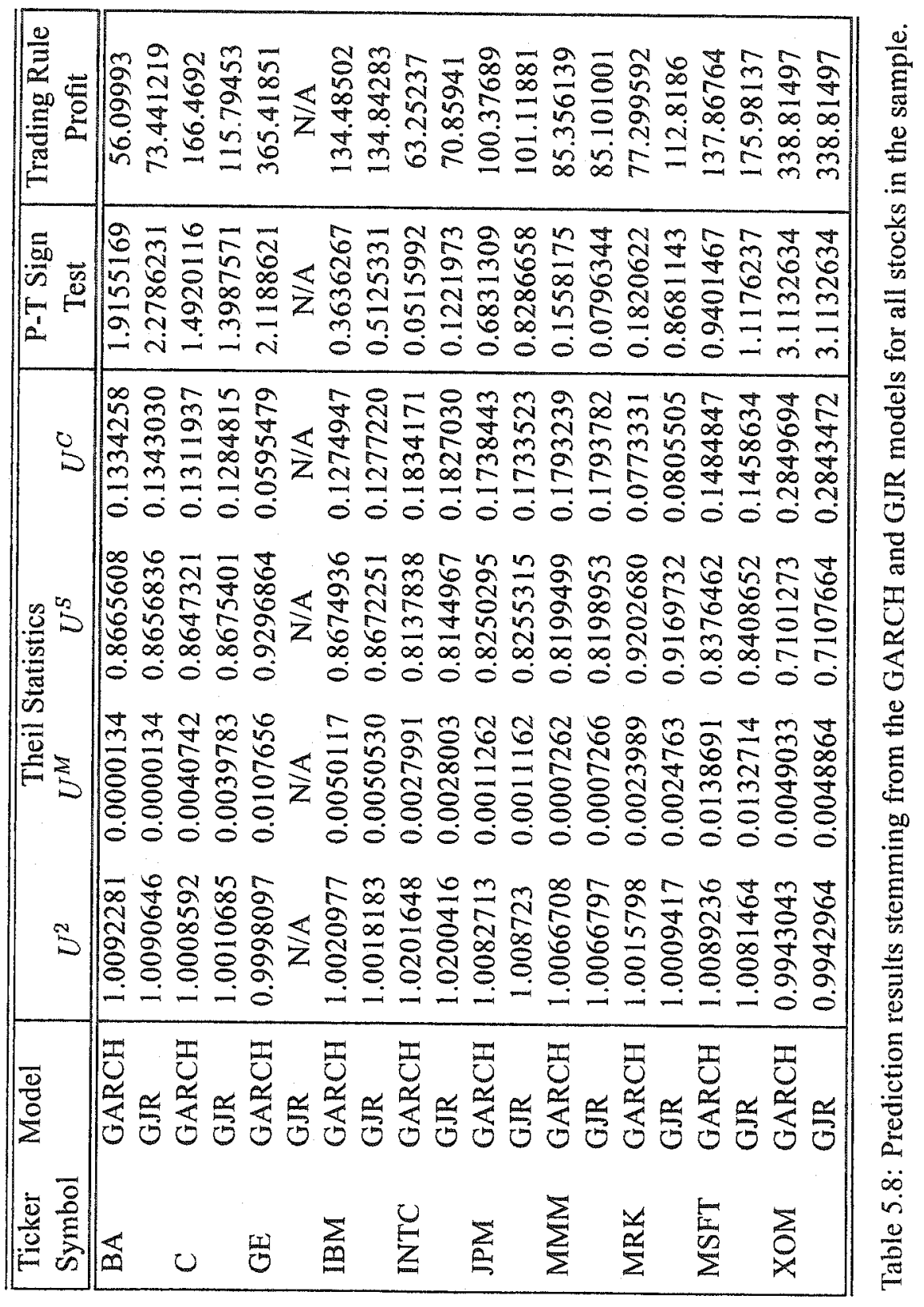


the return measurements are relatively accurate with a variance $R=0.1$. This assumption, made on grounds of tractability, is relaxed in the following analysis.

In reality, it is difficult to guess the level of noise in financial returns. The main analysis has assumed a small level of noise, with an measurement error variance of $R=0.1$. The present sensitivity analysis relaxes this assumption and proposes a series of different values for $R \in\{0.01,0.05,0.2,0.3,0.5,1\}$. Detailed tables of the analysis are presented in Appendix G, Tables G.1-G.10, and parameter estimates are featured in Appendix F, in Tables F.1-F.10.

The results in tables F.1-F.10 show that the differences in likelihoods (LL) for models estimated with different values of $R$ are very small. However, the model parameters tend to vary significantly as a function of $R$. For example, the ARMA parameter values tend to follow a step function with respect to $R$ : as $R$ increases and exceeds a certain threshold, the values of model parameters change drastically, but remain stable once the threshold has been crossed. The log-likelihood values tend to remain level over the whole range of parameter values, however, indicating no improvement in fit.

Another feature concerns the behaviour of $Q$, the system dynamics noise variance, with respect to $R$. As $R$ increases, $Q$ tends to decrease. This can be explained by the fact that as the measurements become less and less reliable ( $R$ increases), the system must rely more and more on its own model of state behaviour and thus assigns its estimates more importance than it would had the measurements been accurate. This tradeoff highlights the standard behaviour of Kalman filter.

Analysis of the summary tables shows that even by varying the level of measurement error variance, the fit of the models is not improved, with Theil statistics remaining mostly unchanged. The statistics which change are the PT and profit statistics. For some stocks, these statistics tend to vary significantly with $R$ for example for stock BA, the higher $R$, the higher the PT and profit statistics (the same behavior can be seen for stocks C, GE, INTC, and MRK; XOM on the other hand displays an inverse relationship). It seems, therefore, that in some stocks the level of measurement noise is higher than for others, and, using a model of returns which discards noisy information leads to better forecasts.

The profitability tests show that trading rule profits were greatest when measurement noise variance was either $R=0.3$ of $R=0.5$, with a return on an equally weighted strategy yielding $88 \%$. This return is higher than the one realized with a preset measurement noise variance of $R=0.1$, for which the trading rule yielded a return of $74 \%$. Increasing the measurement noise variance to $R=1$ resulted in a portfolio return of $73 \%$, which clearly indicates a deterioration in the 
profitability of trading rules when measurements are discounted too much. When the measurement noise levels were set very low, $R=0.01$, the portfolio yielded a retum of only $16 \%$, indicating that underestimating the level of noise in the returns data is detrimental to the predictive ability of the models. The analysis shows, therefore, that, either setting the level of measurement noise variance too low $(R=0.01)$, or, too high $(R=1)$, results in below average predictions, since in either situation, the filter cannot estimate appropriately the value and accuracy of incoming data, and thus make optimal state estimates. This, in turn, results in poor predictions.

\subsection{Further Sensitivity Analysis}

A second stage in the sensitivity analysis consisted in reestimating the models from Table 5.4 to allow the simultaneous estimation of the measurement noise variance $R$ as well as of the driving noise variance $Q$. Table F.11 presents parameter estimates while tables G.1-G.10 feature forecasting results.

Allowing the estimation of all model parameters including $R$ reveals an interesting phenomenon. The measurement noise variance $R$ seems to be much higher than previously assumed: indeed $R>1$ for all models. Interestingly, the system noise variance is very small, on average, $Q=0.2$. This indicates that the model perceives the measurements to be very noisy and is unable to use them very well, and, instead, relies on the internal description of the state in estimating the state of the firm. This also indicates that system state tends to change very slowly over time (small $Q$ ).

Surprisingly, these models tend not to perform as well as the models with preassigned values of measurement noise variance $R$. The trading rule returns for the models featuring simultaneous estimates of $Q$ and $R$ are poor, the trading rule yielding a return of only $33 \%$ for the portfolio. The contrasting results in performance between the models with simultaneously estimated $Q$ and $R$ parameters and the models with the $R$ parameter fixed are perplexing. 


\section{Chapter 6}

\section{Time Varying Parameter Model}

\subsection{Introduction}

This chapter presents an attempt to predict returns using a time-varying parameter version of the univariate model. The approach was motivated by the relative lack of fit to the volatility of returns of models presented to date. The method is based on the Kalman filter and the algorithm developed in the appendix.

The method consists of reestimating model parameters as new measurements arrive, using a moving window of data. The effect of this procedure is to obtain a new parameter estimate whenever a new observation arrives. This procedure can be motivated by arguing that investors reexamine their implicit models of the financial markets as new information is obtained.

As already mentioned, the model is based on a moving window of data. The selection of an appropriate window length is crucial to the success of the experiment, since, by modifying the length of the window of data, it is possible to change the influence of new measurements on the variability of the parameter vector - the shorter the window, the more influence new observations will have on the parameter vector, and the more variable this vector will be. The converse is true as the window length increases. Several window lengths have been tested in order to examine the predictive behaviour of the models. More precisely, five window lengths have been tested, 20,30,50 days, 100, and, finally, 200 days. The windows have been chosen as to reflect the investors' conjectured attention spans in identifying a model of the markets: from one month to almost a year. It is important to mention here that a window of infinite size corresponds to a fixed parameter model as tested in the previous chapter. 


\subsection{Variance Assumptions}

Just like for the fixed parameters models, a variance assumption was made about the behaviour of returns. In particular, it was assumed, that the measurement noise variance $R$, was small, with $R=0.01$. An attempt was made to simultaneously estimate the measurement and system noise variance matrices ( $R$ and $Q$ respectively) in the time-varying framework. Unfortunately, all such attempts (for the different window lengths) resulted in either one of the variance matrices diverging. This example confirms the issues with the simultaneous estimation of the $Q$ and $R$ matrices. It is believed that the estimation problem was mainly caused by the highly heteroscedastic returns data. Consequently, only estimation using a fixed, prespecified, $R$ was performed.

\subsection{Estimation and Results}

The models were estimated with the help of the algorithm presented in the appendix. The initial parameter values were provided by the fixed parameter models. Following the initial variable set-up, predictions and parameter values were obtained in parallel, for each new measurement.

Tables H.1-H.5 (Appendix H, pages 219-221) present prediction results obtained from the time varying models. Several important things can be noted from these tables. First, the Theil statistics $U^{2}$ are high, for all stocks and for all window sizes, exceeding the value of 1 . This indicates the weak ability of the models to predict the changes in prices.

The Theil statistics, however, present other intriguing patterns, especially concerning statistics $U^{S}$ and $U^{C}$. Referring back to the fixed parameter models estimated in the previous chapter, the $U^{S}$ statistics were all close to 1 indicating the models' inability to explain the variance of returns. In the case of the time-varying model with window size of 20 , it can be noted that this statistic is relatively small, having values of 0.4 on average. This indicates that the time-varying model was able to explain the variance of returns much better than the fixed parameter model. This remark applies to all the firms examined in the study as well as all data window sizes. This result is mitigated by the models' relative inability to accurately predict the direction of returns - the statistic $U^{C}$ is close to 0.6 , which is higher than for the fixed parameter model, where this statistic is on average 0.2 .

The P-T sign statistic is significant for one of the stocks, BA, and does not appear to be significant for the rest. This indicates a weak ability of the models to 
predict the sign of returns. The trading rule, on average, realized profits only for two stocks, INTC and MMM.

Figures H.1-H.10 (pages 223-232) display the forecasts achieved using the time varying models for each stock and each estimation window length. As can be seen from the graphs, the shorter the estimation window, the better the models' ability to fit the variance of returns. As the estimation window size increases, the models' ability to fit the data's variability decreases significantly. If the window size were to increase to infinity, the forecasts would be identical to those obtained using the fixed parameter model.

Figures H.11-H.20 (pages 233-242) present the plots of the models' AR and MA parameters over time for all stocks and for three data window sizes (20,50 and 200 measurements). The plots display an expected characteristic: for short estimation window sizes, the parameters tend to vary significantly over time, and, as window size increases, the parameters tend to exhibit a more stable behaviour. Thus parameters vary the most for a window of size 20, and the least for a window of size 200 . For the largest window size (200 observations) the parameters tend to be very stable and, in some cases (C, GE, INTC and XOM), tend to stay close to the values which were obtained using the fixed parameter model.

An unexpected phenomenon was discovered in this analysis: as the data window lengthened, the $U^{S}$ statistic tended to increase while the $U^{C}$ tended to decrease. Thus, it appears that, as the window size lengthens, the models lose their ability to explain the volatility of returns, while they gain ability to explain the direction of price changes. This is confirmed by the P-T statistics which tend to increase with the length of the estimation window. As the data window increases, the trading rule profits also tend to increase - for a window of size of 200 days, 5 out of the 10 stock report a profit, against one for the 20 day window.

These results indicate a tradeoff between the ability of the models to explain the variance of returns, and their ability to predict the direction of returns. As the estimation window lengthens, the $U^{S}$ statistics augment and the $U^{C}$ statistics diminish. Together with this change, one can observe a rise in the magnitudes of the P-T statistics and the trading rule profits.

This behaviour is illustrated in Figures H.21-H.30 (pages 243-252). These figures feature conditional variance forecasts stemming from the $\mathrm{GARCH}$ and GJR-ARCH models estimated previously and the estimated values of the system dynamics noise variance, $Q$, for three data window lengths (20,50 and 200 measurements). The plots show that for small data windows ( 20 observations), the estimated system variance follows very closely the predicted GARCH and GJRARCH variances. This indicates that the time-varying models with short data 
windows can fit very well the variance of returns. As the data window length increases, however, the ability of the models to fit the variance of returns decreases significantly: this can be seen by examining the $Q$ estimate for a window of 200 observations. As expected, the $Q$ estimates for the 50 observations data window lie in between those for window sizes 20 and 200.

An additional feature of the graphs is that the GARCH and GJR-ARCH predicted variances tend to fall-off rapidly after a surge in volatility, while estimates of $Q$ tend to remain high for the length of the estimation window. This artifact is caused by the estimator's smoothing effect.

Another interesting feature of the graphs is that the volatility behaviour of the various stocks tends to be different from stock to stock. Some stocks seem to feature very low volatility (BA and MMM), while for others, large swings in prices tend to occur frequently (MSFT and XOM).

The graphs suggest that the time-varying parameter models' ability to fit the volatility of returns is caused by their ability to properly estimate the true variance of returns. This confirms, therefore, the necessity of modelling the variance of returns in order to better predict their behaviour.

The results on the behaviour of the system variance $Q$ may also yield additional insight on the question of the existence of the variance of returns. Mandelbrot [Mandelbrot, 1963] has argued that sock returns follow a stable Paretian distribution, implying that they do not have a variance. In his analysis, Mandelbrot argued that if it were the case, financial markets would be more volatile than in the situation where returns were Gaussian.

In the results obtained herein, the time varying parameter model shows that the estimated system variance $Q$ tends to decrease in magnitude as the size of the estimation window increases. Thus, when the estimation window size is only 20 observations, the estimates of $Q$ are larger in magnitude and are more variable than when the estimation window size is 200 observations. This behaviour is very well displayed in Figures H.21-H.30 (pages 243-252). Following Mandelbrot's arguments, if returns were distributed according to the stable Paretian distribution, their variance would not exist, and thus estimates of variance would diverge as the sample size would increase. Results obtained in this section show an inverse behaviour: as the estimation sample increases, the estimate of variance $Q$ tends to decrease and stabilize. This evidence contradicts Mandelbrot's hypothesis, and suggests that stock returns are distributed according to a distribution with finite variance. The stable Paretian hypothesis of returns thus does not seem to hold.

The trading rule profits tend to confirm the Theil and the P-T statistics. For a window of size 20 , the model based trading rule registers a loss of $16 \%$ (for a 
portfolio equally invested in the 10 stocks), for a window of size 50 , the trading rule registers a gain of $20 \%$, while for a data window size of 200 observations, the models were able to generate a return of $70 \%$ compared to a retum of $74 \%$ for the fixed parameter model. This progression suggests that the models are able to predict returns better as the estimation window length increases, with the best returns being obtained by the fixed parameter model.

\subsection{An "average" model}

The previous section examined the performance of five time varying parameter models, each based on a different estimation window length. This section reports the results of an experiment which consisted of averaging the forecasts from the different estimation window length models into a single "average" forecast for each stock. The reason behind this experiment is that it is believed that an average of forecasts can yield better results than any of the separate forecasts by themselves.

As such, for each stock, an "average" forecast was computed from the 20 , $30,50,100$, and, finally, 200 days estimation window model forecasts, by simply averaging these forecasts. The performance of the so obtained "average" forecast was then measured. Table H.6 (p. 222) presents the results of the analysis.

A trading rule invested in this "average" model would yield a return of $22 \%$ over the course of the test period. The results show that the "average" model's performance is average. The $U^{2}$ statistics are very close to 1 , but slightly higher than for the window size 200 model, and much lower than those for the window size 20 model. In fact the average model's $U^{2}$ statistics lie just between the statistics for models with windows 50 and 100 . The same can be said for the $U^{S}$ and $U^{C}$ statistics. The $U^{S}$ statistic indicates that the average model cannot fit the volatility of the data as well as the 20 observations window model, but can do so better than the 200 observations window model. The $U^{C}$ statistics for the average model are higher than those for the 200 observations window model and lower than those belonging to the 20 observations window model. Again these statistics lie somewhere between those for the models estimated with the 50 and 100 observations sized windows. The PT test statistics show that the "average" model is not able to better predict the sign of returns than the 200 observations window model.

The profit test results are also mixed. The "average" model never performs better than the model estimated with the longest estimation window length ( 200 observations). However, most of the time, it actually performs better than the 
models estimated with shorter window lengths (100 observations and less). In all, it can be said that the "average" model performs less well than the $200 \mathrm{ob}$ servations window model, but better than the shorter length estimation window models. 


\section{Chapter 7}

\section{Conclusion}

This study examined the predictive ability of stock returns using a new approach modelled after investor behaviour. It was proposed that investors value stocks by estimating their state or condition based on measurements of their performance. It was proposed that this behaviour may be compared to the engineering concept of filtering, in the sense that investors attempt to obtain the most error free estimate of the state of the firm from measurements corrupted by noise. The framework posited that this behaviour may be emulated through the use of the Kalman filter methodology.

Two models were developed to predict the behaviour of returns. The univariate model predicted returns by estimating the state of the firm from historical returns data, while the multivariate model augmented the univariate model through the inclusion of an estimate of the state of the market based on broad economic data. Additionally, a time varying parameter univariate model was also examined.

In order to estimate the proposed models, an algorithm for the estimation of parameters in state space models was derived. The algorithm, based on the maximum likelihood principle, is able to estimate parameters in system matrices of a linear state space system. That is, given a linear state space system,

$$
\begin{array}{r}
z_{t}=H_{t} x_{t}+B_{2, t} u_{2, t}+v_{t}, \\
x_{t+1}=F_{t} x_{t}+B_{1, t} u_{1, t}+G_{t} w_{t}
\end{array}
$$

the algorithm can estimate parameters in any or all of the system matrices $F(\cdot), H(\cdot)$, $B_{1}(\cdot), B_{2}(\cdot)$, including the noise variance matrices $Q(\cdot)$ and $R(\cdot)$. The algorithm was implemented in the SciLab computing environment. 
The proposed models were tested against a set of benchmark models. Those consisted of an AR(1) model for the series of returns, a random walk model, and two types of ARCH models, a GARCH(1,1) model and a GJR-GARCH specifcation.

In the course of the study, it occurred that the originally specified univariate model did not behave properly. Attempts at estimating the originally specified model failed, and several alternative specifications of the model failed to estimate as well. It appears that the main problem consisted in the model's inability to explain the fundamental component of returns. Subsequently the problematic part of the model was dropped, and the univariate model reverted to and $\operatorname{ARMA}(\mathrm{p}, \mathrm{q})$ specification. It is believed that the model's inability to explain the fundamental component of returns is due to the greater variability of that component than originally assumed, and to the presence of larger than expected noise in the data.

The summary of models' performance is presented in Tables G.1-G.10, starting on page 209. The models' predictive performance was tested on 10 stocks selected from the DIA for the years 1997-2000. The results show the models to perform relatively well for their simple form. Trading rules show the models to perform better than the proposed baseline models and the buy-and-hold strategy based on the DJIA index. The models however tend to under-perform a buy-and-hold strategy based on the SP500 and the buy-and-hold strategy based on individually purchasing the 10 stocks in question.

Several problems were noted, the most important of which was the models' inability to fit the high variability of the data. The Theil statistics have shown that the models are simply unable to explain the highly heteroscedastic nature of stock returns. The models' ability to predict the signs of returns was also poor (as shown by the PT statistics), contributing to the relatively poor profitability of the model based trading rules.

In order to remedy this situation, the univariate models were run in a timevarying parameter framework, based on a moving window of data approach. The results of this analysis have shown that it is possible, with a time-varying parameter model framework, to better explain the variance of stock returns. The Theil statistics, as well as graphical analysis, have confirmed that the time varying parameter models' ability to fit the heteroscedastic nature of returns is much greater than that of a fixed parameter model. It appears, however, that the predictive ability is hampered by the time-varying scheme. This is chiefly illustrated by the model based trading rule profits which decrease as the estimation window length decreases. In fact, only the slow changing parameter model (window size of 200 observations) comes close to matching the performance of the fixed-parameter 
model.

It is believed that this decrease in profitability, associated with short estimation window lengths is either caused by over-fitting or by excessive noise in the data. Over-fitting would suggest that short data windows put too much influence on recent observations and not enough on the long-run average. The noise hypothesis on the other hand would suggest that the data is too noisy in the short run for models to be able to estimate reliable parameters.

In order to examine this issue further, an "average" model, which consisted of averaging the predictions from the different length estimation window models, was computed. The analysis showed that the "average" model behaves in a very average manner, having a performance which puts it exactly in between models estimated with long and short length windows. The model can not explain the volatility of returns as well as a short length estimation window model, and cannot predict returns as well as a long estimation window model.

In the end, it seems that a slow varying parameter model (large window size), or, a fixed parameter model may be better able to predict the data than a fast moving parameter model (short window size). The time varying models' ability to fit the heteroscedastic nature of returns highlights, however, the importance of modelling the variance of returns in forecasting studies.

The breakdown of the initial returns model (see Section 5.4.3, p. 103) has several possible sources. The negative variances obtained during the estimation procedure point to model misspecification. The problem is possibly caused by the large amount of noise in the data, and the large volatility of returns. However, the relatively good performance of the time varying parameters model more than makes up for the problems encountered in the estimation of this model.

In all, the study confirms the hypothesis that price data contains information which can be used in predicting the behaviour of returns. The models proposed were able, on average, to outperform both the random walk and the AR(1) baseline models, as well as the GARCH and GJR-GARCH models. This suggests that more complex models would possibly be able to produce better forecasts.

The study has also led to some discoveries concerning the behaviour of the variance of stock returns. First, the study results have shed some light on the question of the existence of variance of stock returns. Mandelbrot [Mandelbrot, 1963], has argued that stock returns follow a stable Paretian distribution, and, thus, that the variance of returns does not exist. Results from the time varying parameter models suggest, to the contrary, that returns variance may exist. The study has found that as the estimation window length increases, the estimated system variance tends to stabilize. If returns were to have no variance, the estimated vari- 
ance from the models would show a divergence as the estimation window size increased. Instead, the estimated variance appears to fluctuate slowly, slower that that for shorter estimation windows, but shows no sign of growing out of bounds. If the estimation window length were to increase beyond the 200 observations used in this study, the estimated variance would stabilize even more, reducing its fluctuations below those that were observed with this window length. This behaviour suggests that the variance of returns is finite in size, and suggests that financial markets are not as volatile as they would be under the stable Paretian hypothesis.

A second discovery concerns the ability of the time-varying-parameter models to estimate the variance of returns. The time-varying-parameter models were used to estimate, among others, the system noise variance $Q$, for the different state space models. It has been found that these estimates reflect very well the heteroscedastic nature of stock returns, and compare favourably with the conditional variance estimates originating from GARCH models. As a comparison, Figures H.21-H.30 (pages 243-252), display the system noise variance $Q$ estimates for selected estimation window lengths and the conditional variance forecasts computed from the GARCH models. It has been discovered that short length window estimates of $Q$ ( 20 and 30 observations) show the greatest resemblance to the GARCH variance estimates. As the window length increases, the estimates of system variance tend to stabilize and do not follow the GARCH estimates as accurately, but still reflect the changing nature of the variance of returns. This result shows that the use of a time varying parameter model may replace the need for a separate ad-hoc analytical model of volatility (like GARCH) to estimate the variance of returns. This is very convenient and shows the strength of the proposed framework. Additionally, the improved, time varying, estimates of the system noise variance $Q$, yield more accurate estimates of the state of the firm (and its variance), than if the system variance $Q$ were assumed fixed.

The third point concerns the levels of noise, or, more precisely, measurement noise present in stock returns data. For the major part of the study, the level of measurement noise present in the returns data was assumed to be low, and the measurement noise variance was preset to $R=0.1$. This value was selected as a reasonable guess as to the level of reliability of returns: a low variance indicating that returns are relatively error free. The predictions using this assumption have been shown to perform relatively well. However, to analyze the effects of different assumptions about magnitudes of measurement noise, a sensitivity analysis was run, in which predictions were performed for different levels of measurement noise variance $R$, ranging from $R=0.01$ to $R=1$. Prediction results have shown 
that best performance is achieved when the measurement noise variance is in the range of $R=0.3$ to $R=0.5$, with values outside of this range yielding slightly worse results. When the measurement noise and system noise variance statistics, $Q$ and $R$, were estimated simultaneously, the $Q$ statistics have been shown to be lower compared to fixed $R$ models, and the $R$ statistics have been shown to be higher. While such a model could have been expected to perform better than a model with preimposed values of measurement level noise, the model has performed rather poorly, with trading rule returns being below average.

The reason for the underperformance of these models is the fact that the $R$ estimates are too high: a high estimated measurement noise variance causes the models to discount the measurements too heavily, forcing the filter to rely on its internal model for the state in computing state estimates. This results in below average state estimates, and poor predictions. The results show that the best predictions have been given by models with moderate amounts of measurement noise variance $R(0.3 \leq R \leq 0.5)$. The implications are that returns do include information useful in predicting future behaviour, the reverse having been true, the returns would be totally discounted. This in turn suggests that returns have inherently, only moderate amounts of noise in them, and are not all useless noise. Following returns can thus bring some benefits to the careful analyst.

The study sheds new light on the question of market efficiency: returns are not all noise, but contain information useful for making predictions. While they contain information, however, it is difficult to extract it using simple models and to make effective predictions. The models seem unable to outperform buy-andhold strategies based on investing in individual stocks, but they can perform better than the simple baseline models, and better than some index based buy-and-hold strategies. Returns, however, contain information useful for prediction, and, this is also confirmed by the fact that the best performing models are the ones with only small amounts of measurement noise.

The question is whether models can be constructed which outperform the buyand-hold strategies on a cost adjusted basis. Part of the answer is provided by the Theil statistic: the models (except time-varying models) have difficulty in explaining the volatile nature of stock returns. The use of models which capture more of the heteroscedasticity in returns may hold the key to improving predictive accuracy.

The principal problem encountered in the study seems to be the lack of fit due to the models' inability to explain the volatility of returns. The sensitivity analysis has shown that this remains a problem for a range of parameter values. Furthermore, the use of ARCH type models did not help in explaining the volatile 
nature of returns. This question remains unresolved and deserves further study. The predictive ability of the models thus stems solely from their ability to predict the sign of stock returns.

The study also points to the importance of parameter estimates in successfully predicting market behaviour, and in particular, the importance of the knowledge of the magnitudes of the measurement and driving noise variances in the model ( $R$ and $Q$ respectively). The study has shown returns to contain moderate amounts of noise. In order to predict the markets successfully, investors need to have correct estimates of the parameter values, and in particular, the values of variances $Q$ and $R$, since wrong choices for these statistics will undeniably damage the predictive power of the investors' model. Indeed, if an investor were to construe a higher estimate of measurement noise than one which is present in returns in reality, he would discount information contained in returns as more unreliable than it really is. This would result in suboptimal state estimates since the states would not be updated with useful information contained in returns. If an investor were, on the other hand, to construe that measurement noise is smaller than it really is, then his model would follow the noise in the returns, and again, his state estimates would be suboptimal. This shows that it is crucial for investors to effectively know (or, indeed, to estimate) the real accuracy of the data they are using, since mistaken perceptions of data reliability can lead to serious investment mistakes.

In examining this study, one should keep in mind that while the proposed framework strives to explain and emulate investor behaviour, the proposed model cannot fully copy the investors' analytical processes. Indeed, the predictive devices presented in the work are only models of investor behaviour, and thus do not capture the richness of the whole decision making process that all investors go through when making investments. In fact, the models proposed herein are deficient in two principal ways. First, the models do not capture all of the analytical capacity of an investor, and, represent, in fact, only a small portion of an investor's cognitive palette. In order to capture more of an investor's intellectual skills, the model's complexity would have to be increased, as would be the case, for example, if one were to attempt to explain the heteroscedastic nature of returns, or attempt to interpret corporate financial statements. The second weakness of the models comes from the fact that an empirical model cannot grasp all the variety of data available to the investor. In fact, an empirical model can only use a fraction of the data available to an investor, and can use subjective data only with difficulty. The investor, however, is not bound by these limitations, and only needs to take care not to be overwhelmed with the quantity of data available to him.

As such, the models used in this study are limited mathematically, and do not 
make use of all possible types of data. In order to better explain the behaviour of returns, the models would have to be expanded in order to include more firm specific data as well as include broader economic measures. This presents several challenges. The first challenge consists in recognizing that many firm specific and economic variables are not available at daily intervals of time. Furthermore, such variables may be difficult to analyze, and specifying a mathematical model for treating this data may be very difficult. However, concentrating on stock returns mitigates some of these concerns, since the different types of information are continuously being reflected in stock returns, as investors process the incoming data. Thus the proposed models, implicitly operate on a wide array of data, if only indirectly.

The results of the study seem, therefore, to favour the hypothesis that returns contain information useful for their prediction. The models proposed cannot however exploit all information provided in historical records to better predict the future. The study shows the weakness of the conventional modelling of returns, but suggests that more advanced techniques such as those involving heteroscedasticity, nonlinearities, and, non-Gaussian residuals could potentially provide better forecasts.

The framework proposed in the study is advantageous in this respect. Not only does it attempt to emulate the behaviour of market participants in valuing stocks, but it is also easily extended to include more complex valuation processes. It is a very flexible modelling tool, and yet it provides a theoretical framework for researchers studying market behaviour.

Future investigations should take advantage of the state-space framework and should center on explaining several important aspects of the behaviour of returns. First, they should attempt to explain the high volatility of returns, which is, seemingly, the principal reason for the relatively poor fit to the data. Secondly, different models for the distribution of returns should be examined. This should include a re-examination of the levels of noise in information available to investors. Finally, the proposed method allows the markets to be considered as a single system. This aspect of the method should be exploited in order to construct a better model of investor and market behaviour which will allow for a global look at financial markets. 


\section{Appendix A}

\section{Data}

The stock market data consists of daily closing stock prices for 10 firms randomly selected from the 30 firms composing the Dow Jones Industrial Average index as of January 2000. Data spans the time period from January 1, 1990 to January 1, 2000. The data are collected from the free historical data source $f$ nance.yahoo.com. The historical prices are adjusted for stock splits and dividends. Table A. 1 lists the selected firms and includes information as to the beginning and end of the price record for each furm

For the purposes of the study, the data was divided into an estimation and a forecasting sample. The estimation sample spans from January 1, 1990 to December 31,1996 , and consists of 1770 daily returns, while, the forecasting sample spans the remainder of the data, (January 1, 1997 to January 1, 2000) and contains 760 observations.

Stock returns are computed as percentage changes in the levels of closing prices from one trading day to another (the weekend return is defined as the percentage change in price from Friday's close to Monday's close). Denoting $P_{i, t}$ as the closing price for firm $i$ on day $t$, the return $r_{i, t}$ is computed as follows:

$$
r_{i, t}=100 * \frac{P_{i, t}-P_{i, t-1}}{P_{i, t-1}}
$$

Daily end-of-day values for the DI Composite, FTSE100 and NIKKEI225 stock market indices have also been collected, and index returns have been computed. Table A.2 presents the data used in the study.

The economic data used in the study are the short term interest rates in the form of 3 month US T-Bills, long term interest rates in the form of 30 year bond data, and high risk corporate bond yields consisting of bonds rated BAA by Moody's. 
The data spans the period from January 1, 1990 to January 1,2000 and originates from the Federal Reserve at www.chicagofed.org.

Exchange rate data used in the study consists of "noon buying rates in New York City certified by the Federal Reserve Bank of New York for customs purposes for cable transfers payable in foreign currencies". The data are the Dollar/Pound exchange rate, the Dollar/Yen exchange rate, and the implied exchange rate for the Yen/Pound. Exchange rate returns are computed in the same manner as returns for stocks. Table A.3 presents more information as to the data used in the study. The data is collected from the Federal Reserve at www.chicagofed.org. 


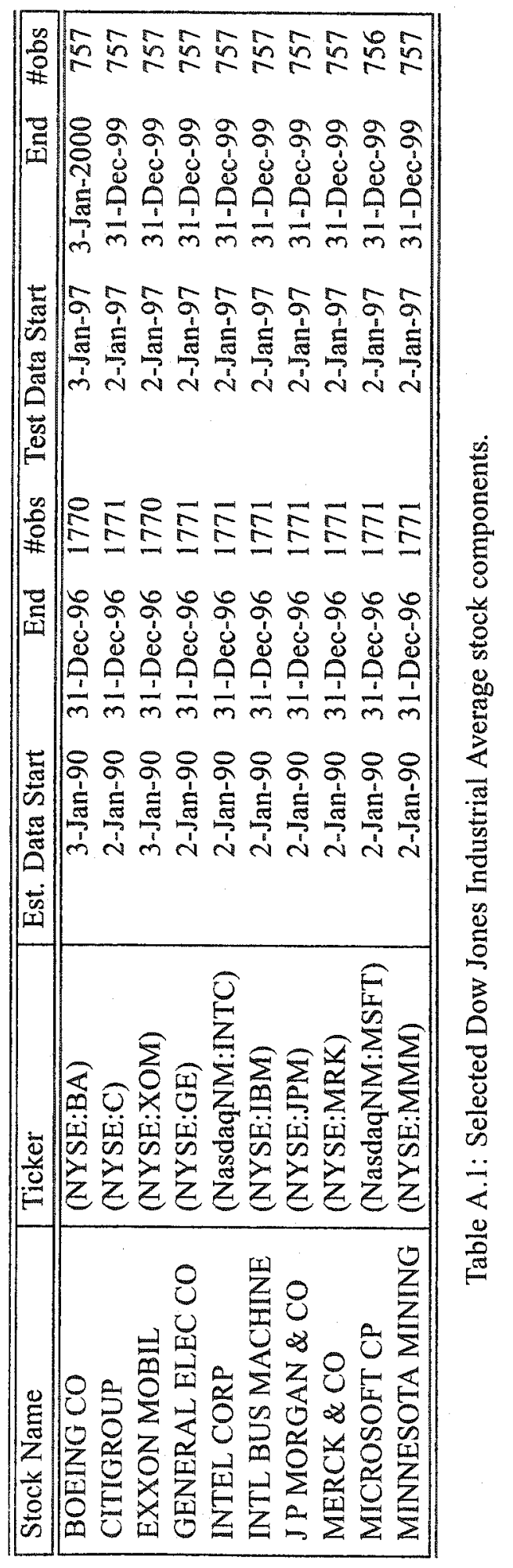




\begin{tabular}{|l|l|l|l|}
\hline \hline Country & Index Name & Ticker & Data Start - Data End \\
\hline \hline USA & DJ 65 Composite & ADJA & 1-Jan-1990 - 1-Jan-2000 \\
UK & FTSE 100 & -FTSE & 1-Jan-1990 - 1-Jan-2000 \\
Japan & Nikkei 225 & -N225 & 1-Jan-1990 - 1-Jan-2000 \\
\hline
\end{tabular}

Table A.2: Selected stock indices used in the study.

\begin{tabular}{|c|l|c|}
\hline \hline Exchange Rate & Type & Data Start - Data End \\
\hline \hline US / Pound & quoted & 1-Jan-1990-1-Jan-2000 \\
US / Yen & quoted & 1-Jan-1990-1-Jan-2000 \\
Pound / Yen & implied & 1-Jan-1990-1-Jan-2000 \\
\hline
\end{tabular}

Table A.3: Selected exchange rate data used in the study. 


\section{Appendix B}

\section{Estimation Algorithm}

\section{B.1 Introduction}

This appendix presents an algorithm developed for the estimation of parameters in linear state space models. The algorithm and approach are based on the work of Maybeck [Maybeck, 1979, Maybeck, 1981, Maybeck, 1972]. Assume the following general system description for the process $z$ :

$$
\begin{aligned}
x\left(t_{i+1}\right) & =F\left(t_{i}\right) x\left(t_{i}\right)+B_{1}\left(t_{i}\right) u_{1}\left(t_{i}\right)+G\left(t_{i}\right) w\left(t_{i}\right) \\
z\left(t_{i}\right) & =H\left(t_{i}\right) x\left(t_{i}\right)+B_{2}\left(t_{i}\right) u_{2}\left(t_{i}\right)+v\left(t_{i}\right),
\end{aligned}
$$

where $x(\cdot)$ is the system state, and $w(\cdot)$ and $v(\cdot)$ respectively represent independent Gaussian system and observation noises with zero means and with variances:

$$
\begin{aligned}
& \mathbb{E}\left[w\left(t_{i}\right) w^{T}\left(t_{j}\right)\right]=Q\left(t_{i}\right) \delta_{i, j} \\
& \mathbb{E}\left[v\left(t_{i}\right) v^{T}\left(t_{j}\right)\right]=R\left(t_{i}\right) \delta_{i, j}
\end{aligned}
$$

The initial state conditions are not known with precision and are assumed to be normally distributed with mean $\mathbb{E}\left[x\left(t_{0}\right)\right]=\hat{x}_{0}$ and variance,

$$
\mathbb{E}\left[\left[x\left(t_{0}\right)-\hat{x}_{0}\right]\left[x\left(t_{0}\right)-\hat{x}_{0}\right]^{T}\right]=P_{0} .
$$

When the system is described completely, that is, the matrices $F(\cdot), H(\cdot)$, $B_{1}(\cdot), B_{2}(\cdot), Q(\cdot)$ and $R(\cdot)$ are known, one can use the Kalman filter to estimate the state of the system at any point in time. Unfortunately, in many situations, system parameters are known only with limited precision. It is necessary then to 
infer the values of these unknown parameters directly from the behavior of the system under study. This can be achieved through state estimation and system identification techniques. This appendix presents an approach to the estimation of state space model parameters based on the principle of maximum likelihood.

\section{B.2 The Method}

It is assumed here that the system described above is well defined except for a number $p$ of unknown parameters which can belong to any or all of the system matrices $F(\cdot), H(\cdot), B_{1}(\cdot), B_{2}(\cdot), Q(\cdot)$ or $R(\cdot)$. The proposed technique considers the simultaneous estimation of states and parameters. Let $\theta\left(t_{i}\right)$ be the combined vector of states and parameters to be estimated, and, $\mathcal{Z}_{i}$ represent system observations. The goal is to find the optimal value of $\theta\left(t_{i}\right)^{*}$ such as to maximize the likelihood function $L\left[\theta\left(t_{i}\right), \mathcal{Z}_{i}\right]$. The optimum is found by differentiating the likelihood function with respect to the vector of parameters:

$$
\left.\frac{\partial L\left[\theta\left(t_{i}\right), \mathcal{Z}_{i}\right]}{\partial \theta\left(t_{i}\right)}\right|_{\theta\left(t_{i}\right)=\theta\left(t_{i}\right)^{\circ}}=0^{T}
$$

Maybeck ([Maybeck, 1981], p. 76) proposes the following likelihood function for the simultaneous estimation of states and parameters: $\ln f_{x\left(t_{i}\right), Z\left(t_{i}\right) \mid a}\left(\xi, \mathcal{Z}_{i} \mid \alpha\right)$, where $x\left(t_{i}\right)$ is the state vector, $Z\left(t_{i}\right)$ is the history of measurements at time $t_{i}$, and $a$ is the $p$-dimensional vector of parameters. This likelihood equation can be broken down as follows into components easily obtained through the Kalman filter:

$$
\begin{aligned}
f_{x\left(t_{i}\right), Z\left(t_{i}\right) \mid a} & =f_{x\left(t_{i}\right) \mid Z\left(t_{i}\right), a} \cdot f_{Z\left(t_{i}\right) \mid a} \\
& =f_{x\left(t_{i}\right) \mid Z\left(t_{i}\right), a} f_{z\left(t_{i}\right) \mid Z\left(t_{i-1}\right), a} f_{Z\left(t_{i-1}\right) \mid a} \\
& =f_{x\left(t_{i}\right) \mid Z\left(t_{i}\right), a} \prod_{j=1}^{i} f_{z\left(t_{j}\right) \mid Z\left(t_{j-1}\right), a}
\end{aligned}
$$

where the last term in the development at time $j=1$ is $f_{z\left(t_{1}\right) \mid a}(\zeta \mid \alpha)$. The individual components can be easily obtained from the Kalman filter. First,

$$
\begin{aligned}
f_{x\left(t_{i}\right) \mid Z\left(t_{i}\right), a}\left(\xi \mid \mathcal{Z}_{i}, \alpha\right) & =\frac{1}{(2 \pi)^{n / 2}\left|P\left(t_{i}^{+}\right)\right| 1 / 2} \\
& \times \exp \left\{-\frac{1}{2}\left[\xi-\hat{x}\left(t_{i}^{+}\right)\right]^{T} P\left(t_{i}^{+}\right)^{-1}\left[\xi-\hat{x}\left(t_{i}^{+}\right)\right]\right\}
\end{aligned}
$$


and,

$$
\begin{aligned}
f_{z\left(t_{j}\right) \mid Z\left(t_{j-1}\right), a}\left(\zeta_{j} \mid \mathcal{Z}_{j-1}, \alpha\right) & =\frac{1}{(2 \pi)^{m / 2}\left|A\left(t_{j}\right)\right|^{1 / 2}} \\
& \times \exp \left\{-\frac{1}{2}\left[\zeta_{j}-H\left(t_{j}\right) \hat{x}\left(t_{j}^{-}\right)-B_{2}\left(t_{j}\right) u_{2}\left(t_{j}\right)\right]^{T} A\left(t_{j}\right)^{-1}\right. \\
& \left.\times\left[\zeta_{j}-H\left(t_{j}\right) \hat{x}\left(t_{j}^{-}\right)-B_{2}\left(t_{j}\right) u_{2}\left(t_{j}\right)\right]\right\},
\end{aligned}
$$

where $\hat{x}\left(t_{i}^{+}\right), P\left(t_{i}^{+}\right), \hat{x}\left(t_{j}^{-}\right), P\left(t_{j}^{-}\right)$and $A\left(t_{j}\right)$ are implicitly dependent on the parameter values, and $A\left(t_{j}\right)=H\left(t_{j}\right) P\left(t_{j}^{-}\right) H\left(t_{j}\right)^{T}+R\left(t_{j}\right)$. The complete likelihood function can be written as:

$$
\begin{aligned}
\ln f_{x\left(t_{i}\right), Z\left(t_{i}\right) \mid a}\left(\xi, Z_{i} \mid \alpha\right) & =-\frac{n+i m}{2} \ln (2 \pi)-\frac{1}{2} \ln \left(\left|P\left(t_{i}^{+}\right)\right|\right)-\frac{1}{2} \sum_{j=1}^{i} \ln \left(\left|A\left(t_{j}\right)\right|\right) \\
& -\frac{1}{2}\left[\xi-\hat{x}\left(t_{i}^{+}\right)\right]^{T} P\left(t_{i}^{+}\right)^{-1}\left[\xi-\hat{x}\left(t_{i}^{+}\right)\right] \\
& -\frac{1}{2} \sum_{j=1}^{i}\left[\zeta_{j}-H\left(t_{j}\right) \hat{x}\left(t_{j}^{-}\right)-B_{2}\left(t_{j}\right) u_{2}\left(t_{j}\right)\right]^{T} A\left(t_{j}\right)^{-1} \\
& \times\left[\zeta_{j}-H\left(t_{j}\right) \hat{x}\left(t_{j}^{-}\right)-B_{2}\left(t_{j}\right) u_{2}\left(t_{j}\right)\right]
\end{aligned}
$$

The derivatives of the likelihood function with respect to individual components $\alpha_{k}$ of the parameter vector $\alpha$ are as follows:

$$
\begin{aligned}
& -2 \frac{\partial \ln f_{x\left(t_{i}\right), Z\left(t_{i}\right) \mid a}\left(\xi, \mathcal{Z}_{i} \mid \alpha\right)}{\partial \alpha_{k}} \\
& =\operatorname{tr}\left[P\left(t_{i}^{+}\right)^{-1} \frac{\partial P\left(t_{i}^{+}\right)}{\partial \alpha_{k}}\right] \\
& +\sum_{j=1}^{i} \operatorname{tr}\left[A\left(t_{j}\right)^{-1} \frac{\partial A\left(t_{j}\right)}{\partial \alpha_{k}}\right]-2\left[\frac{\partial \hat{x}\left(t_{i}^{+}\right)}{\partial \alpha_{k}}\right]^{T} P\left(t_{i}^{+}\right)^{-1}\left[\xi-\hat{x}\left(t_{i}^{-}\right)\right] \\
& -\quad\left[\xi-\hat{x}\left(t_{i}^{+}\right)\right]^{T} P\left(t_{i}^{+}\right)^{-1} \frac{\partial P\left(t_{i}^{+}\right)}{\partial \alpha_{k}} P\left(t_{i}^{+}\right)^{-1}\left[\xi-\hat{x}\left(t_{i}^{+}\right)\right] \\
& -\quad 2 \sum_{j=1}^{i}\left[\frac{\partial H\left(t_{j}\right)}{\partial \alpha_{k}} \hat{x}\left(t_{i}^{-}\right)+H\left(t_{j}\right) \frac{\partial \hat{x}\left(t_{i}^{-}\right)}{\partial \alpha_{k}}+\frac{\partial B_{2}\left(t_{j}\right)}{\partial \alpha_{k}} u_{2}\left(t_{j}\right)\right]^{T} A\left(t_{j}\right)^{-1} \\
& \times \quad\left[\zeta_{j}-H\left(t_{j}\right) \hat{x}\left(t_{i}^{-}\right)-B_{2}\left(t_{j}\right) u_{2}\left(t_{j}\right)\right]
\end{aligned}
$$




$$
\begin{aligned}
& -\sum_{j=1}^{i}\left[\zeta_{j}-H\left(t_{j}\right) \hat{x}\left(t_{i}^{-}\right)-B_{2}\left(t_{j}\right) u_{2}\left(t_{j}\right)\right]^{T} A\left(t_{j}\right)^{-1} \frac{\partial A\left(t_{j}\right)}{\partial \alpha_{k}} A\left(t_{j}\right)^{-1} \\
\times & {\left[\zeta_{j}-H\left(t_{j}\right) \hat{x}\left(t_{i}^{-}\right)-B_{2}\left(t_{j}\right) u_{2}\left(t_{j}\right)\right] }
\end{aligned}
$$

When $\alpha=a^{*}\left(t_{i}\right)$ then $\xi=\hat{x}\left(t_{i}^{+}\right)$, and therefore terms such as $\left[\xi-\hat{x}\left(t_{i}^{+}\right)\right]$in the above equation reduce to 0 . Taking this into account, the likelihood equation can be written as follows:

$$
\begin{aligned}
& \operatorname{tr}\left[P\left(t_{i}^{+}\right)^{-1} \frac{\partial P\left(t_{i}^{+}\right)}{\partial \alpha_{k}}\right] \\
- & 2 \sum_{j=1}^{i}\left[\frac{\partial H\left(t_{j}\right)}{\partial \alpha_{k}} \hat{x}\left(t_{i}^{-}\right)+H\left(t_{j}\right) \frac{\partial \hat{x}\left(t_{i}^{-}\right)}{\partial \alpha_{k}}+\frac{\partial B_{2}\left(t_{j}\right)}{\partial \alpha_{k}} u_{2}\left(t_{j}\right)\right]^{T} A\left(t_{j}\right)^{-1} r_{j} \\
+ & \left.\sum_{j=1}^{i} \operatorname{tr}\left\{\left[A\left(t_{j}\right)^{-1}-A\left(t_{j}\right)^{-1} r_{j} r_{j}^{T} A\left(t_{j}\right)^{-1}\right] \frac{\partial A\left(t_{j}\right)}{\partial \alpha_{k}}\right\}\right|_{\alpha=a^{*}\left(t_{i}\right)}=0,
\end{aligned}
$$

where,

$$
r_{j}=z_{j}-H\left(t_{j}\right) \hat{x}\left(t_{i}^{-}\right)-B_{2}\left(t_{j}\right) u_{2}\left(t_{j}\right)
$$

Using the Kalman filter and simultaneously solving $p$ of these equations will yield maximum likelihood estimates of the states and parameters of the state space system. Unfortunately the likelihood equations do not have a closed form solution and an iterative solution must be considered. Maybeck [Maybeck, 1981] proposes a solution based on Rao's scoring method, where new parameter estimates $\hat{\theta}\left(t_{i}\right)^{*}$ are obtained as successive corrections to older estimates:

$$
\hat{\theta}\left(t_{i}\right)^{*}=\hat{\theta}\left(t_{i}\right)_{*}+J\left[t_{i}, \hat{\theta}\left(t_{i}\right)_{*}\right]^{-1} \frac{\partial L\left[\theta\left(t_{i}\right)_{*}, Z_{i}\right]^{T}}{\partial \theta},
$$

where $J\left[t_{i}, \hat{\theta}\left(t_{i}\right)_{*}\right]$ is the conditional information matrix and $\left\{\partial L\left[\theta\left(t_{i}\right)_{*}, Z_{i}\right] / \partial \theta\right\}^{T}$ is the score vector. The implementation of the algorithm is performed by first decomposing the $\mathrm{N}$-step score into single measurement scores $s^{1}\left[Z_{j}, \hat{a}\left(t_{i}\right)_{*}\right]$ and a "final term" $\gamma^{1}\left[Z_{i}, \hat{a}\left(t_{i}\right)_{*}\right]$ as follows:

$$
\frac{\partial L\left[\hat{x}\left(t_{i}\right)_{*}, \hat{a}\left(t_{i}\right)_{*}, Z_{i}\right]}{\partial a_{k}}=\gamma^{1}\left[Z_{i}, \hat{a}\left(t_{i}\right)_{*}\right]+\sum_{j=1}^{i} s^{1}\left[Z_{j}, \hat{a}\left(t_{i}\right)_{*}\right]
$$


where,

$$
\begin{aligned}
s^{1}\left[Z_{j}, \hat{a}\left(t_{i}\right)_{*}\right]= & {\left[\frac{\partial H\left(t_{j}\right)}{\partial \alpha_{k}} \hat{x}\left(t_{i}^{-}\right)+H\left(t_{j}\right) \frac{\partial \hat{x}\left(t_{i}^{-}\right)}{\partial \alpha_{k}}+\frac{\partial B_{2}\left(t_{j}\right)}{\partial \alpha_{k}} u_{2}\left(t_{j}\right)\right]^{T} A\left(t_{j}\right)^{-1} r_{j} } \\
- & \frac{1}{2} \operatorname{tr}\left(\left[A\left(t_{j}\right)^{-1}-A\left(t_{j}\right)^{-1} r_{j} r_{j}^{T} A\left(t_{j}\right)^{-1}\right] \frac{\partial A\left(t_{j}\right)}{\partial \alpha_{k}}\right) \\
& \gamma^{1}\left[Z_{i}, \hat{a}\left(t_{i}\right)_{*}\right]=-\frac{1}{2} \operatorname{tr}\left[P\left(t_{i}^{+}\right)^{-1} \frac{\partial P\left(t_{i}^{+}\right)}{\partial \alpha_{k}}\right]
\end{aligned}
$$

The conditional information matrix can also be decomposed into components, and a closed form solution is provided in Maybeck [Maybeck, 1981, Maybeck, 1972].

$$
\begin{aligned}
J\left[t_{i}, \hat{x}\left(t_{i}\right)_{*}, \hat{a}\left(t_{i}\right)_{*}\right] & =\mathbb{E}\left[\gamma_{k}\left[Z\left(t_{i}\right), a\right] \gamma_{l}\left[Z\left(t_{i}\right), a\right] \mid a=a_{t}\right] \\
& +\sum_{j=1}^{i} \mathbb{E}\left[s_{k}^{1}\left[Z\left(t_{j}\right), a\right] s_{l}^{1}\left[Z\left(t_{j}\right), a\right] \mid a=a_{t}\right]
\end{aligned}
$$

where,

$$
\begin{aligned}
& \mathbb{E}\left[s_{k}^{1}\left[Z\left(t_{j}\right), a\right] s_{l}^{1}\left[Z\left(t_{j}\right), a\right] \mid a=a_{t}\right] \\
&= \frac{1}{2} \operatorname{tr}\left[A\left(t_{j}\right)^{-1} \frac{\partial A\left(t_{j}\right)}{\partial \alpha_{k}} A\left(t_{j}\right)^{-1} \frac{\partial A\left(t_{j}\right)}{\partial \alpha_{l}}\right] \\
&+ {\left[\hat{x}\left(t_{i}^{-}\right)^{T} \frac{\partial H\left(t_{j}\right)^{T}}{\partial \alpha_{k}}+\frac{\partial \hat{x}\left(t_{i}^{-}\right)^{T}}{\partial \alpha_{k}} H\left(t_{j}\right)^{T}+u_{2}\left(t_{j}\right)^{T} \frac{\partial B_{2}\left(t_{j}\right)^{T}}{\partial \alpha_{k}}\right] A\left(t_{j}\right)^{-1} } \\
& \times {\left[\frac{\partial H\left(t_{j}\right)}{\partial \alpha_{l}} \hat{x}\left(t_{i}^{-}\right)+H\left(t_{j}\right) \frac{\partial \hat{x}\left(t_{i}^{-}\right)}{\partial \alpha_{l}}+\frac{\partial B_{2}\left(t_{j}\right)}{\partial \alpha_{l}} u_{2}\left(t_{j}\right)\right], \text { and, } } \\
& \mathbb{E}\left[\gamma_{k}\left[Z\left(t_{i}\right), a\right] \gamma_{l}\left[Z\left(t_{i}\right), a\right] \mid a=a_{t}\right]=\frac{1}{2} \operatorname{tr}\left[P\left(t_{i}^{+}\right)^{-1} \frac{\partial P\left(t_{i}^{+}\right)}{\partial \alpha_{k}} P\left(t_{i}^{+}\right)^{-1} \frac{\partial P\left(t_{i}^{+}\right)}{\partial \alpha_{l}}\right] \\
&+\frac{\partial \hat{x}\left(t_{i}^{+}\right)^{T}}{\partial \alpha_{k}} P\left(t_{i}^{+}\right)^{-1} \frac{\partial \hat{x}\left(t_{i}^{+}\right)}{\partial \alpha_{l}}
\end{aligned}
$$

The equations B.19 and B.20 were obtained using the approximation proposed in Maybeck [Maybeck, 1981] on page 89. 


\section{B.3 The Algorithm}

The algorithm aims at finding the best value of the parameter vector $a$ over the sample of measurements of size $N$. The initial conditions for the recursions are as follows:

$$
\begin{aligned}
\hat{x}\left(t_{0}^{+}\right) & =\text {as previously computed } \\
P\left(t_{0}^{+}\right) & =\text {as previously computed } \\
\frac{\partial \hat{x}\left(t_{0}^{+}\right)}{\partial \alpha_{k}} & =0 \\
\frac{\partial P\left(t_{0}^{+}\right)}{\partial \alpha_{k}} & =0
\end{aligned}
$$

At the most recent measurement time $t_{i}$, starting with the last best parameter estimate $\hat{a}_{*}\left(t_{i}\right)$, a new, updated parameter estimate $\hat{a}^{*}\left(t_{i}\right)$ is computed by the $\mathrm{N}$-step recursion consisting of the Kalman filter recursions and additional computations designed to evaluate the score and the conditional information matrix. At the end of the iteration process a new parameter estimate can be computed through:

$$
\hat{a}^{*}\left(t_{i}\right)=\hat{a}_{*}\left(t_{i}\right)+J\left[t_{i}, \hat{x}\left(t_{i}\right)_{*}, \hat{a}\left(t_{i}\right)_{*}\right]^{-1} \frac{\partial L\left[\hat{x}\left(t_{i}\right)_{*}, \hat{a}\left(t_{i}\right)_{*}, Z_{i}\right]^{T}}{\partial a}
$$

The steps in the iteration process are described next.

\section{B.3.1 Kalman propagation step}

The first step in the recursion process is the propagation of the state through the Kalman equations.

$$
\begin{aligned}
\hat{x}\left(t_{j}^{-}\right) & =F\left(t_{j}\right) \hat{x}\left(t_{j-1}^{+}\right)+B_{1}\left(t_{j-1}\right) u_{1}\left(t_{j-1}\right) \\
P\left(t_{j}^{-}\right) & =F\left(t_{j}\right) P\left(t_{j-1}^{+}\right) F\left(t_{j}\right)^{T}+G\left(t_{j-1}\right) Q\left(t_{j}\right) G\left(t_{j-1}\right)^{T} \\
A\left(t_{j}\right) & =H\left(t_{j}\right) P\left(t_{j}^{-}\right) H\left(t_{j}\right)^{T}+R\left(t_{j}\right) \\
K\left(t_{j}\right) & =P\left(t_{j}^{-}\right) H\left(t_{j}\right)^{T} A\left(t_{j}\right)^{-1}
\end{aligned}
$$




\section{B.3.2 Score equations (propagation step)}

In order to evaluate the equations corresponding to the score vector, it is necessary to have knowledge of certain matrices such as $\frac{\partial F\left(t_{j}\right)}{\partial \alpha_{k}}$ etc... The following expressions are evaluated for all $p$ unknown parameters:

$$
\begin{aligned}
\frac{\partial \hat{x}\left(t_{j}^{-}\right)}{\partial \alpha_{k}} & =F\left(t_{j}\right) \frac{\partial \hat{x}\left(t_{j-1}^{+}\right)}{\partial \alpha_{k}}+\frac{\partial F\left(t_{j}\right)}{\partial \alpha_{k}} \hat{x}\left(t_{j-1}^{+}\right)+\frac{\partial B_{1}\left(t_{j-1}\right)}{\partial \alpha_{k}} u_{1}\left(t_{j-1}\right)\left(B \left(t_{j}\right.\right. \\
\frac{\partial P\left(t_{j}^{-}\right)}{\partial \alpha_{k}} & =\frac{\partial F\left(t_{j}\right)}{\partial \alpha_{k}} P\left(t_{j-1}^{+}\right) F\left(t_{j}\right)^{T}+F\left(t_{j}\right) \frac{\partial P\left(t_{j-1}^{+}\right)}{\partial \alpha_{k}} F\left(t_{j}\right)^{T} \\
& +F\left(t_{j}\right) P\left(t_{j-1}^{+}\right) \frac{\partial F\left(t_{j}\right)^{T}}{\partial \alpha_{k}}+G\left(t_{j}\right) \frac{\partial Q\left(t_{j-1}\right)}{\partial \alpha_{k}} G\left(t_{j}\right)^{T} \\
\frac{\partial A\left(t_{j}\right)}{\partial \alpha_{k}} & =\frac{\partial H\left(t_{j}\right)}{\partial \alpha_{k}} P\left(t_{j}^{-}\right) H\left(t_{j}\right)^{T}+H\left(t_{j}\right) \frac{\partial P\left(t_{j}^{-}\right)}{\partial \alpha_{k}} H\left(t_{j}\right)^{T} \\
& +H\left(t_{j}\right) P\left(t_{j}^{-}\right) \frac{\partial H\left(t_{j}\right)^{T}}{\partial \alpha_{k}}+\frac{\partial R\left(t_{j}\right)}{\partial \alpha_{k}}
\end{aligned}
$$

\section{B.3.3 Conditional information matrix equations (propagation step)}

At this point in the recursions, the conditional information matrix can be evaluated using equation B.19.

$$
\begin{aligned}
& \mathbb{E}\left[s_{k}^{1}\left[Z\left(t_{j}\right), a\right] s_{l}^{1}\left[Z\left(t_{j}\right), a\right] \mid a=a_{t}\right] \\
= & \frac{1}{2} \operatorname{tr}\left[A\left(t_{j}\right)^{-1} \frac{\partial A\left(t_{j}\right)}{\partial \alpha_{k}} A\left(t_{j}\right)^{-1} \frac{\partial A\left(t_{j}\right)}{\partial \alpha_{l}}\right] \\
+ & {\left[\hat{x}\left(t_{i}^{-}\right)^{T} \frac{\partial H\left(t_{j}\right)^{T}}{\partial \alpha_{k}}+\frac{\partial \hat{x}\left(t_{i}^{-}\right)^{T}}{\partial \alpha_{k}} H\left(t_{j}\right)^{T}+u_{2}\left(t_{j}\right)^{T} \frac{\partial B_{2}\left(t_{j}\right)^{T}}{\partial \alpha_{k}}\right] A\left(t_{j}\right)^{-1} } \\
\times & {\left[\frac{\partial H\left(t_{j}\right)}{\partial \alpha_{l}} \hat{x}\left(t_{i}^{-}\right)+H\left(t_{j}\right) \frac{\partial \hat{x}\left(t_{i}^{-}\right)}{\partial \alpha_{l}}+\frac{\partial B_{2}\left(t_{j}\right)}{\partial \alpha_{l}} u_{2}\left(t_{j}\right)\right] }
\end{aligned}
$$

\section{B.3.4 Kalman updating step}

For convenience and computational purposes, define the following quantities:

$$
r_{j}=z_{j}-H\left(t_{j}\right) \hat{x}\left(t_{j}^{-}\right)-B_{2}\left(t_{j}\right) u_{2}\left(t_{j}\right)
$$




$$
\begin{aligned}
D\left(t_{j}\right) & =I-K\left(t_{j}\right) H\left(t_{j}\right) \\
n_{j} & =A\left(t_{j}\right)^{-1} r_{j} \\
C\left(t_{j}\right) & =A\left(t_{j}\right)^{-1}-n_{j} n_{j}^{T}
\end{aligned}
$$

The Kalman filter state updating equations are (Maybeck [Maybeck, 1981] p. 87 suggests the use of the Joseph form for the state covariance matrix):

$$
\begin{aligned}
& \hat{x}\left(t_{j}^{+}\right)=\hat{x}\left(t_{j}^{-}\right)+K\left(t_{j}\right) r_{j} \\
& P\left(t_{j}^{+}\right)=D\left(t_{j}\right) P\left(t_{j}^{-}\right) D\left(t_{j}\right)^{T}+K\left(t_{j}\right) R\left(t_{j}\right) K\left(t_{j}\right)^{T}
\end{aligned}
$$

\section{B.3.5 Score equations (updating step)}

For each unknown parameter $k=1, \ldots, p$, the following score related equations are evaluated:

$$
\begin{aligned}
s^{1}\left[Z_{j}, \hat{a}\left(t_{i}\right)_{*}\right] & =\left[\frac{\partial H\left(t_{j}\right)}{\partial \alpha_{k}} \hat{x}\left(t_{j}^{-}\right)+H\left(t_{j}\right) \frac{\partial \hat{x}\left(t_{j}^{-}\right)}{\partial \alpha_{k}}+\frac{\partial B_{2}\left(t_{j}\right)}{\partial \alpha_{k}} u_{2}\left(t_{j}\right)\right]^{T} n_{j} \\
& -\frac{1}{2} \operatorname{tr}\left[C\left(t_{j}\right) \frac{\partial A\left(t_{j}\right)}{\partial \alpha_{k}}\right] \\
\frac{\partial \hat{x}\left(t_{j}^{+}\right)}{\partial \alpha_{k}} & =D\left(t_{j}\right)\left[\frac{\partial \hat{x}\left(t_{j}^{-}\right)}{\partial \alpha_{k}}+\left(\frac{\partial P\left(t_{j}^{-}\right)}{\partial \alpha_{k}} H\left(t_{j}\right)^{T}+P\left(t_{j}^{-}\right) \frac{\partial H\left(t_{j}\right)^{T}}{\partial \alpha_{k}}\right) n_{j}\right] \\
& -K\left(t_{j}\right) \frac{\partial H\left(t_{j}\right)}{\partial \alpha_{k}} \hat{x}\left(t_{j}^{+}\right)-K\left(t_{j}\right) \frac{\partial B_{2}\left(t_{j}\right)}{\partial \alpha_{k}} u_{2}\left(t_{j}\right) \\
& -K\left(t_{j}\right) \frac{\partial R\left(t_{j}\right)}{\partial \alpha_{k}} n_{j} \\
\frac{\partial P\left(t_{j}^{+}\right)}{\partial \alpha_{k}} & =D\left(t_{j}\right) \frac{\partial P\left(t_{j}^{-}\right)}{\partial \alpha_{k}} D\left(t_{j}\right)^{T}-P\left(t_{j}^{+}\right) \frac{\partial H\left(t_{j}\right)^{T}}{\partial \alpha_{k}} K\left(t_{j}\right)^{T} \\
& -K\left(t_{j}\right) \frac{\partial H\left(t_{j}\right)}{\partial \alpha_{k}} P\left(t_{j}^{+}\right)+K\left(t_{j}\right) \frac{\partial R\left(t_{j}\right)}{\partial \alpha_{k}} K\left(t_{j}\right)^{T}
\end{aligned}
$$

\section{B.3.6 Conditional information matrix equations (updating step)}

At this point, the remaining component of the conditional information matrix represented by equation B. 20 is evaluated.

$$
\mathbb{E}\left[\gamma_{k}\left[Z\left(t_{i}\right), a\right] \gamma_{l}\left[Z\left(t_{i}\right), a\right] \mid a=a_{t}\right]=\frac{1}{2} \operatorname{tr}\left[P\left(t_{i}^{+}\right)^{-1} \frac{\partial P\left(t_{i}^{+}\right)}{\partial \alpha_{k}} P\left(t_{i}^{+}\right)^{-1} \frac{\partial P\left(t_{i}^{+}\right)}{\partial \alpha_{l}}\right]
$$




$$
+\frac{\partial \hat{x}\left(t_{i}^{+}\right)^{T}}{\partial \alpha_{k}} P\left(t_{i}^{+}\right)^{-1} \frac{\partial \hat{x}\left(t_{i}^{+}\right)}{\partial \alpha_{l}}
$$

Finally, the remaining component of the score vector is to be evaluated:

$$
\gamma^{1}\left[Z_{i}, \hat{a}\left(t_{i}\right)_{*}\right]=-\frac{1}{2} \operatorname{tr}\left[P\left(t_{i}^{+}\right)^{-1} \frac{\partial P\left(t_{i}^{+}\right)}{\partial \alpha_{k}}\right]
$$

At the end of the recursion process, after all $N$ measurements have been treated, an estimate of the parameter vector can be computed using expression B.26. Once this estimate is obtained, the Kalman filter can be used to process further measurements. 


\section{Appendix $\mathbf{C}$}

\section{Univariate Model}

Daily returns $r_{i, t}$ for every firm $i$, on the stock market can be decomposed into two components: a real return $r_{i, t}^{\text {fund }}$, and an imaginary return $r_{i, t}^{\text {nfund }}$, as follows:

$$
r_{i, t}=r_{i, t}^{\text {fund }}+r_{i, t}^{\text {nfund }}+v_{i, t}, \quad \operatorname{Var}\left(v_{i, t}\right)=\sigma_{v_{i}}^{2}
$$

where $v_{i, t}$ is an white noise error term with mean zero and a specific variance.

\section{C.1 The Fundamental Component}

The fundamental return for each company $i$ is hypothesized to follow the damped linear trend model of Harvey p. 46 [Harvey, 1989], having the following form:

$$
\begin{aligned}
r_{i, t}^{\text {fund }} & =r_{i, t-1}^{\text {fund }}+g_{i, t}+\eta_{i, t}^{(1)} & & \operatorname{Var}\left(\eta_{i, t}^{(1)}\right)=\sigma_{\eta_{i}^{(1)}}^{2} \\
g_{i, t} & =\rho g_{i, t-1}+\eta_{i, t}^{(2)} & \operatorname{Var}\left(\eta_{i, t}^{(2)}\right) & =\sigma_{\eta_{i}^{(2)}}^{2}
\end{aligned}
$$

where $0 \leq \rho \leq 1$ is the damping coefficient. When $\rho=1$, the model reduces to the simple linear trend model as proposed by Harrison and Stevens [Harrison and Stevens, 1976]. The residual terms $\eta_{i, t}^{(1)}$ and $\eta_{i, t}^{(2)}$, are mutually uncorrelated white noise disturbances of zero mean, and variances respectively $\sigma_{\eta_{i}^{(1)}}^{2}$ and $\sigma_{\eta_{i}^{(2)}}^{2}$. The state space representation of the model writes:

$$
\left[\begin{array}{c}
r_{i, t}^{\text {fund }} \\
g_{i, t}
\end{array}\right]=\left[\begin{array}{cc}
1 & \rho \\
0 & \rho
\end{array}\right]\left[\begin{array}{c}
r_{i, t-1}^{\text {fund }} \\
g_{i, t-1}
\end{array}\right]+\left[\begin{array}{ll}
1 & 1 \\
0 & 1
\end{array}\right]\left[\begin{array}{l}
\eta_{i, t}^{(1)} \\
\eta_{i, t}^{(2)}
\end{array}\right] .
$$




\section{C.2 The Daily Component}

The day-of-the-week effect is modeled according to the method of Harvey [Harvey, 1989]. There are assumed to exist $w=5$ types of days during a week: Tuesday, Wednesday, Thursday, Friday, and Weekend, this last one stretching from the Friday close to the Monday close. For each day, $j=$ Tue, ..., Fri, the seasonal effect associated with that day depends on its past values: $d_{j, t}=$ $d_{j, t-1}+\chi_{j, t}$. The daily effects, $d_{j, t}$, are modeled as a seasonality of a period of one week, where the seasonality cancels out, $d_{\text {Weekend,t }}=-\sum_{j=\text { Tuesday }}^{\text {Friday }} d_{j, t}$, requiring a vector of size $w-1=4$ to describe it. Let $d_{i, t}$ be a $(w-1) \times 1$ vector representing the daily effects for firm $i$ and let $\chi_{i, t}$ be the $(w-1) \times 1$ vector of respective disturbances. Then, the transition equation for the day-of-the-week effect can be written as: $d_{i, t}=d_{i, t-1}+\chi_{i, t}$, the variance of $\chi_{i, t}$ being written as (see Harvey p.43):

$$
\operatorname{Var}\left(\chi_{i, t}\right)=\sigma_{\chi}^{2}\left[I_{4}-\frac{1}{5} O_{4}\right]=\sigma_{\chi}^{2} \times\left[\begin{array}{cccc}
4 / 5 & -1 / 5 & -1 / 5 & -1 / 5 \\
-1 / 5 & 4 / 5 & -1 / 5 & -1 / 5 \\
-1 / 5 & -1 / 5 & 4 / 5 & -1 / 5 \\
-1 / 5 & -1 / 5 & -1 / 5 & 4 / 5
\end{array}\right]
$$

where $\sigma_{\chi}^{2}$ is the variance of the disturbance terms, and $O_{4}$ is the unity matrix of size 4. In order to reflect daily effects, the measurement equation is modified to include a time-varying coefficient matrix $D_{t}$, changing over time to reflect the day of the week:

$$
r_{i, t}=r_{i, t}^{\text {fund }}+r_{i, t}^{\text {nfund }}+D_{t} d_{i, t}+\epsilon_{i, t} \text {. }
$$

In this setup, $D_{t}$ is a dummy variable vector of size $1 \times(w-1)$ which varies systematically over time to pick the right daily effect from the vector $d_{i, t}$ - during any day, from Tuesday to Friday, $D_{t}$ contains a 1 in the corresponding position, and zeros elsewhere, while during a Weekend, $D_{t}$ contains -1 in all positions. For example, the coefficient matrix $D_{t}$ for Monday has the form $D_{t}=[1,0,0,0]$, for Tuesday, it is $D_{t}=[0,1,0,0]$, while for Friday it is $D_{t}=[-1,-1,-1,-1]$.

\section{C.3 Non-fundamental Component}

The univariate model further assumes that the non-fundamental component of returns can be modeled as an ARA(p,q) model in the following manner:

$$
r_{i, t}^{\text {nfund }}=\phi_{1} r_{i, t-1}^{\text {nfund }}+\ldots+\phi_{p} r_{i, t-p}^{\text {nfund }}+\psi_{1} \omega_{i, t-1}+\ldots+\psi_{q} \omega_{i, t-q}+\omega_{t}
$$


Letting $r=\max (p, q+1)$, (and $\phi_{j}=0$ if $j>p$, and $\omega_{j}=0$ for $\left.j>q\right)$, the transition equation in the state space representation of the ARMA(p,q) model is:

$$
\xi_{t}=\left[\begin{array}{ccccc}
\phi_{1} & \phi_{2} & \cdots & \cdots & \phi_{r} \\
1 & 0 & \cdots & 0 & 0 \\
0 & \ddots & 0 & \vdots & \vdots \\
\vdots & 0 & \ddots & 0 & 0 \\
0 & \cdots & 0 & 1 & 0
\end{array}\right] \times \xi_{t-1}+\left[\begin{array}{c}
\omega_{t} \\
0 \\
\vdots \\
0
\end{array}\right]
$$

where, $\xi_{t}$ is the state vector of size $(r \times 1)$, while the measurement equation takes the following form:

$$
\begin{aligned}
r_{i, t}^{\text {nfund }} & =\left[1, \psi_{1}, \ldots, \psi_{r-1}\right] \xi_{t}+v_{i, t} \\
& =H^{\text {ARMA }} \xi_{t}+v_{i, t}
\end{aligned}
$$

\section{C.4 The Complete Model}

Returns observed on the market are hypothesized to have two components: a fundamental return component $r_{i, t}^{\text {fund }}$, an non-fundamental return component $r_{i, t}^{\text {nfund }}$ plus an innovation term $v_{i, t}$. The fundamental part follows a damped linear trend model and the non-fundamental part is composed of two components - the day of the week effect, and, an $\operatorname{ARMA}(p, \dot{q})$ stationary component describing investor behaviour unrelated to the real performance of the firm. Taken together, these three components, fundamental growth, non-fundamental fluctuations, and seasonality, yield the following model representation:

$$
\begin{aligned}
r_{i, t} & =r_{i, t}^{\text {fund }}+r_{i, t}^{\text {nfund }}+v_{i, t} \\
& =r_{i, t}^{\text {fund }}+H^{\text {ARMA }} \xi_{t}+D_{t} d_{t}+v_{i, t}
\end{aligned}
$$

where $H^{\mathrm{ARMA}}$ represents the vector of coefficients for the ARMA model, and $D_{t}$ represents the daily dummy variables. The complete representation of the univariate model for company $i$, in state space form, follows next (the index $i$ is omitted for a clearer notation). The measurement equation for the univariate model is a linear combination of the three components, while, the transition equation describes the evolution of the state $x_{t}=\left[r_{t}^{\text {fund }}, g_{t}, d_{t, \text { Tue }}, d_{t, \text { Wed }}, d_{t, \text { Thu }}, d_{t, \mathrm{~W} / \mathrm{E}}, \xi_{t}^{1}, \xi_{t}^{2}, \cdots, \xi_{t}^{r}\right]$ of the individual firms. The two equations are as follows: 


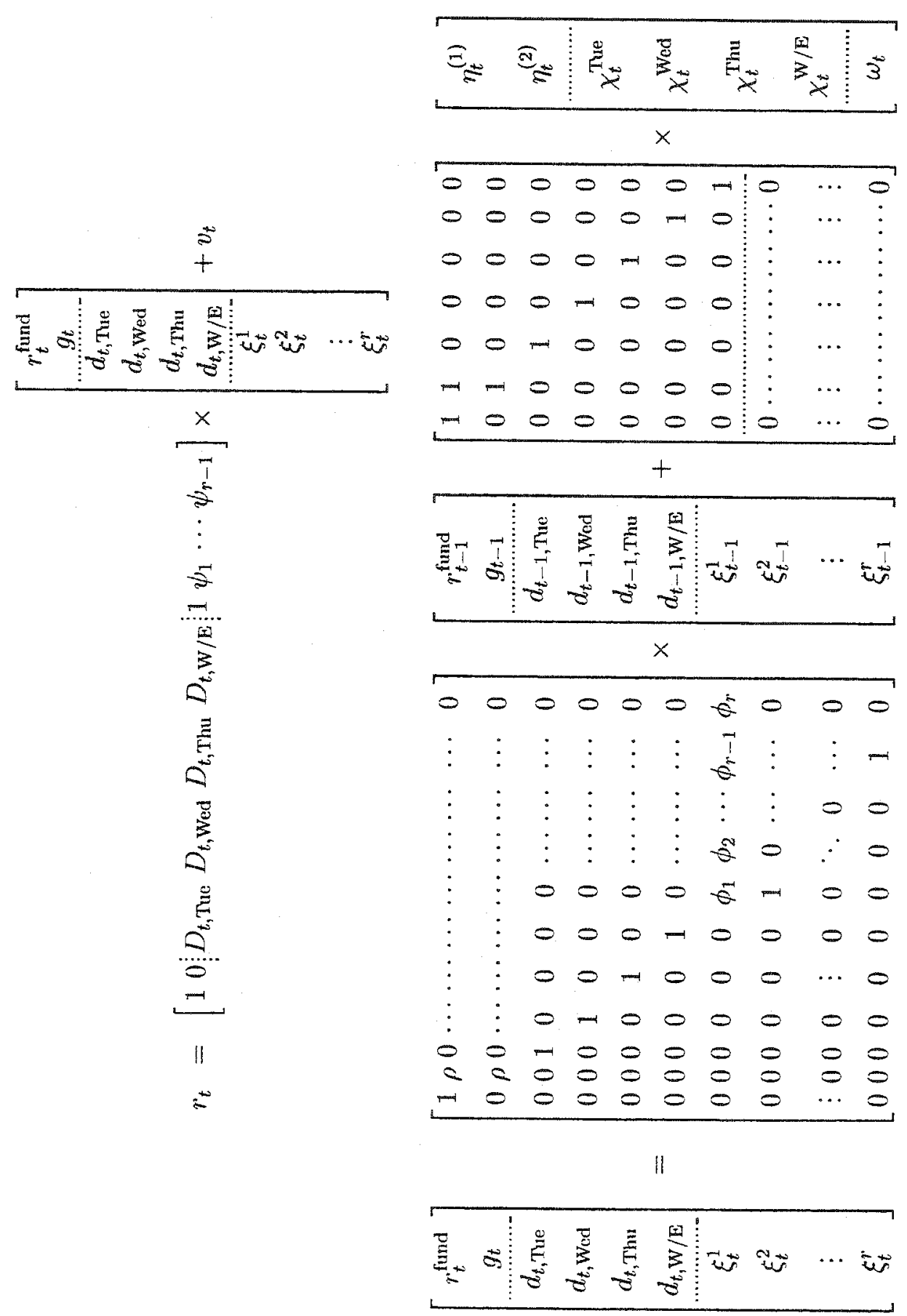


The measurement and transition matrices $H$ and $F$, are, respectively, of sizes $1 \times(6+r)$ and $(6+r) \times(6+r)$ - the measurement matrix $H$ relates the state of the firm to its returns, while the transition matrix describes the behaviour of the state of the firm through time. The system noise vector $w_{t}$ is composed of 7 elements and is shaped through the noise input matrix $G$ of size $(6+r) \times 7$. The measurement error $v_{i, t}$ is scalar, has zero mean and variance $R=\mathbb{E}\left[v_{i, t} v_{i, t}^{T}\right]=\sigma_{v, \hat{i}}^{2}$. The transition equation error covariance matrix has the following structure:

$$
\begin{aligned}
Q_{t} & =\mathbb{E}\left[w_{t} w_{t}^{T}\right] \\
& =\left[\begin{array}{ccccccc}
\sigma_{\eta^{(1)}}^{2} & 0 & 0 & 0 & 0 & 0 & 0 \\
0 & \sigma_{\eta^{(2)}}^{2} & 0 & 0 & 0 & 0 & 0 \\
0 & 0 & 4 / 5 \sigma_{\chi}^{2} & -1 / 5 \sigma_{\chi}^{2} & -1 / 5 \sigma_{\chi}^{2} & -1 / 5 \sigma_{\chi}^{2} & 0 \\
0 & 0 & -1 / 5 \sigma_{\chi}^{2} & 4 / 5 \sigma_{\chi}^{2} & -1 / 5 \sigma_{\chi}^{2} & -1 / 5 \sigma_{\chi}^{2} & 0 \\
0 & 0 & -1 / 5 \sigma_{\chi}^{2} & -1 / 5 \sigma_{\chi}^{2} & 4 / 5 \sigma_{\chi}^{2} & -1 / 5 \sigma_{\chi}^{2} & 0 \\
0 & 0 & -1 / 5 \sigma_{\chi}^{2} & -1 / 5 \sigma_{\chi}^{2} & -1 / 5 \sigma_{\chi}^{2} & 4 / 5 \sigma_{\chi}^{2} & 0 \\
0 & 0 & 0 & 0 & 0 & 0 & \sigma_{\omega}^{2}
\end{array}\right] .
\end{aligned}
$$


Appendix D

Univariate Model Results 


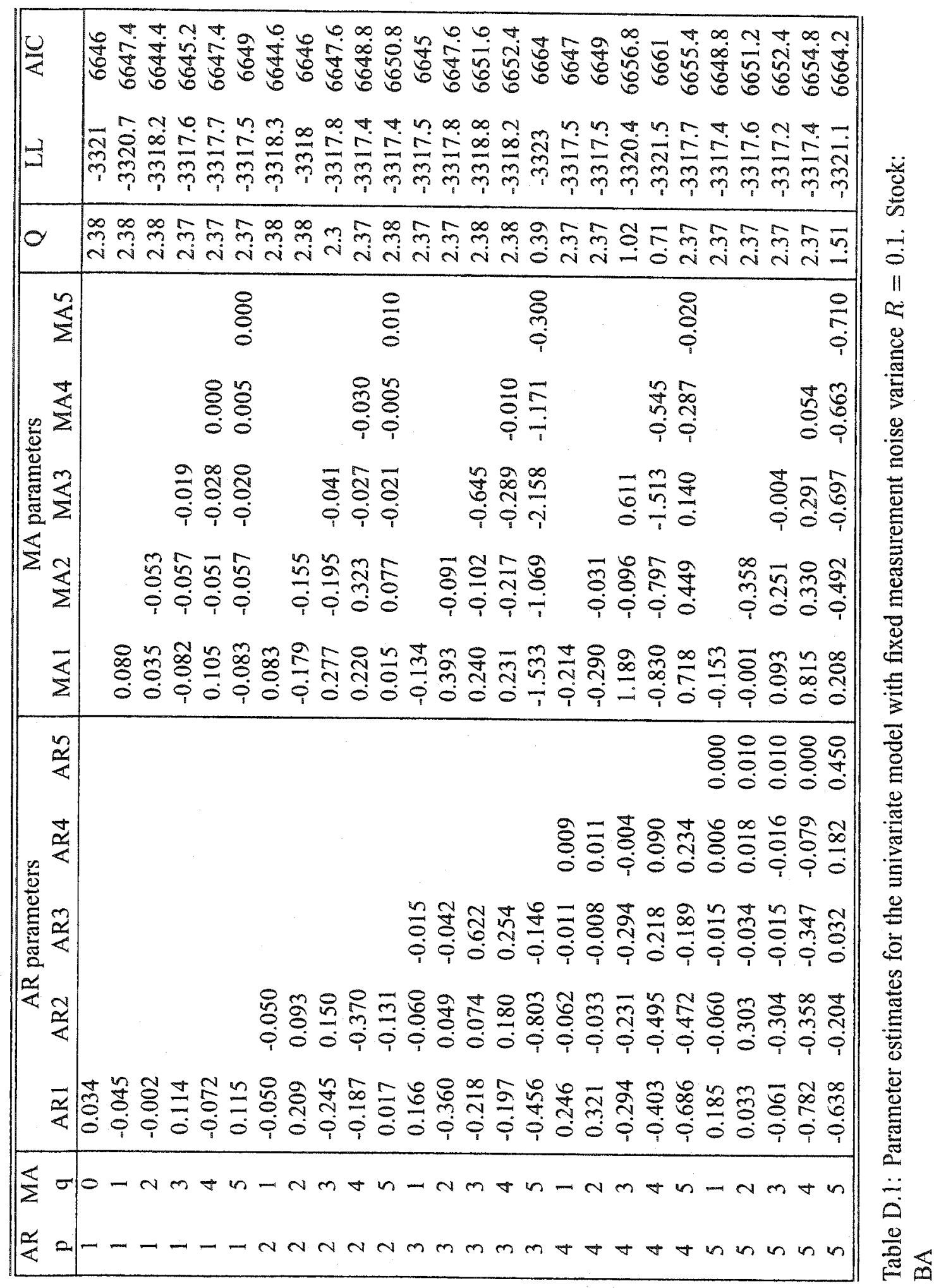




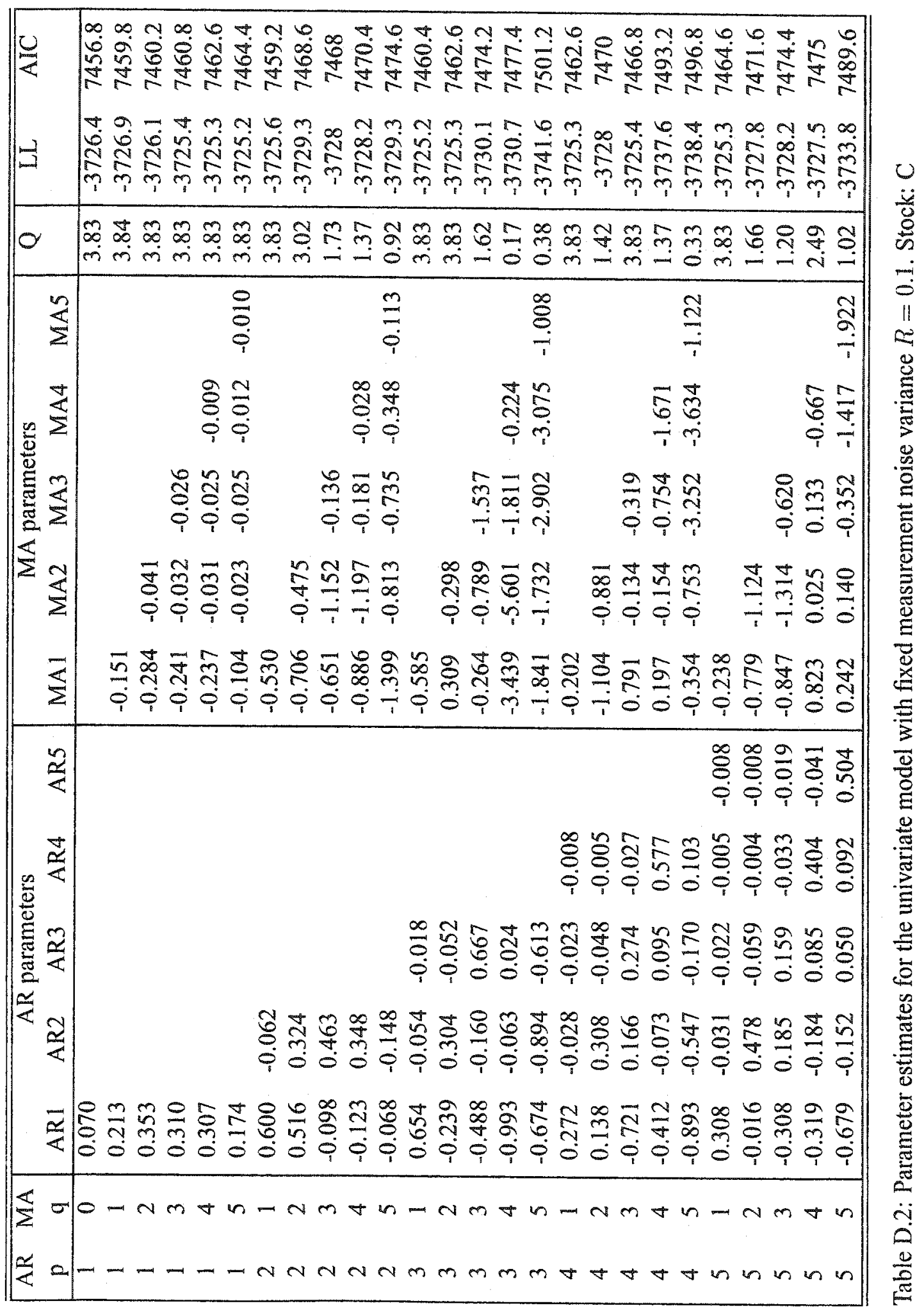




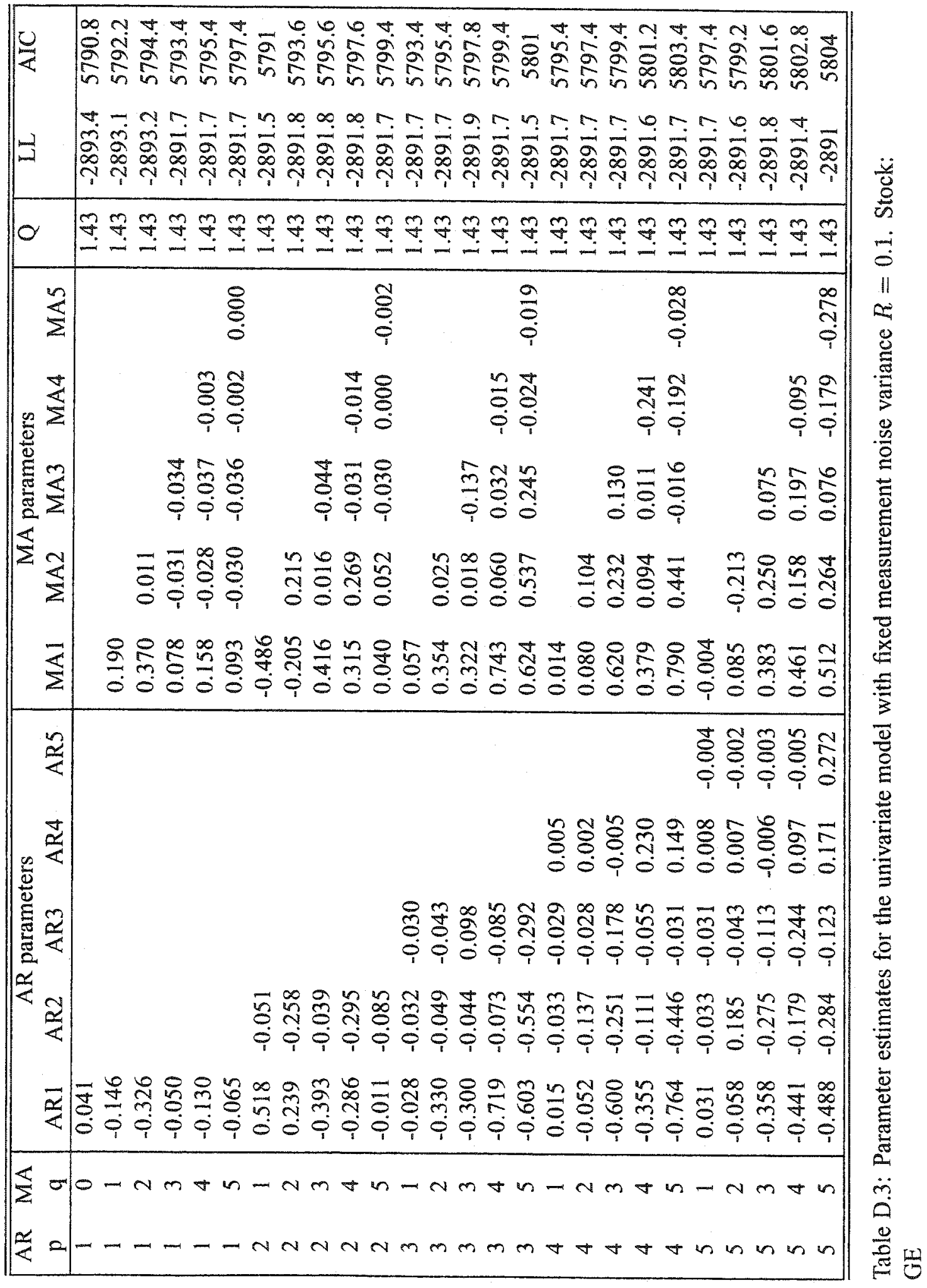




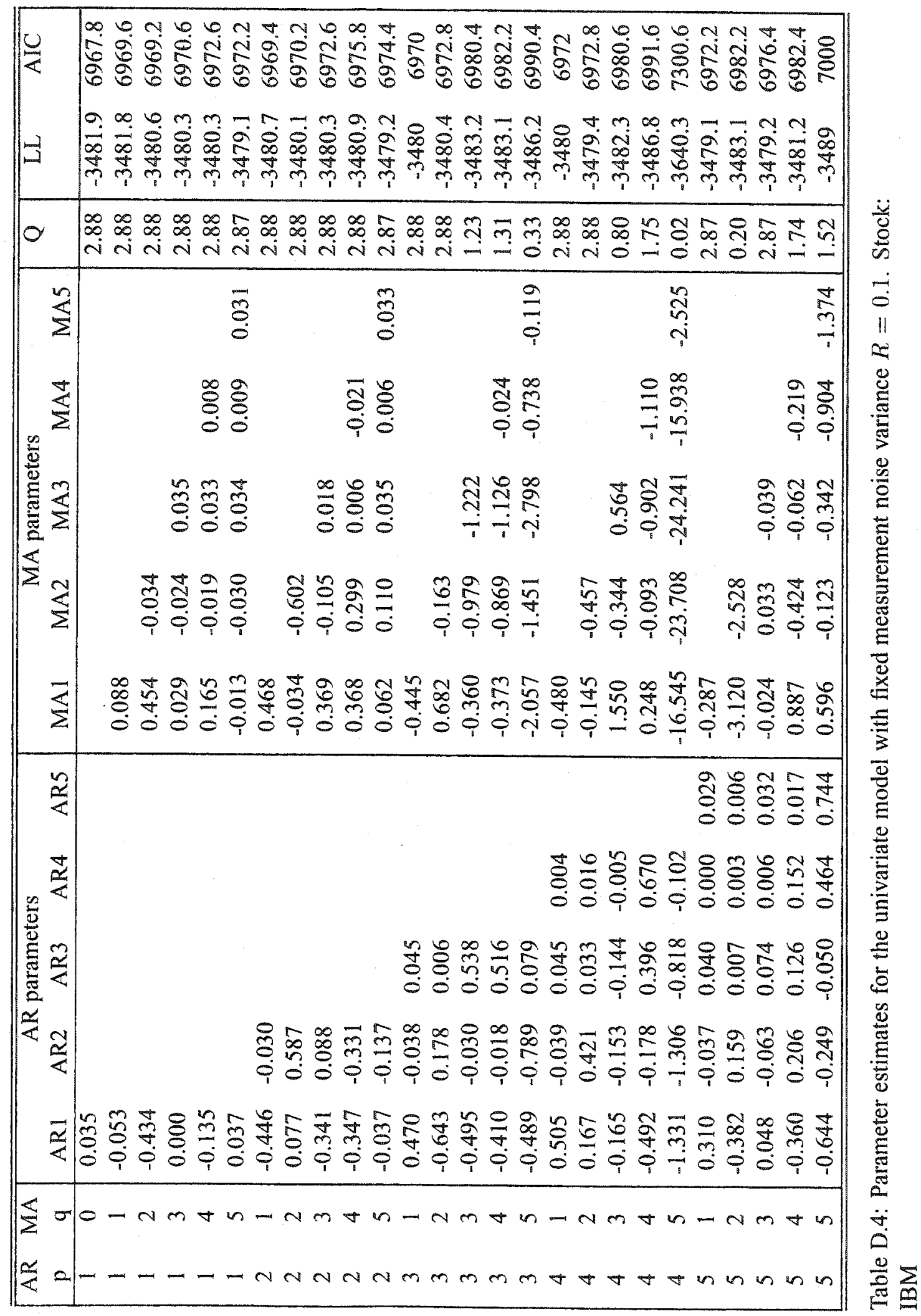




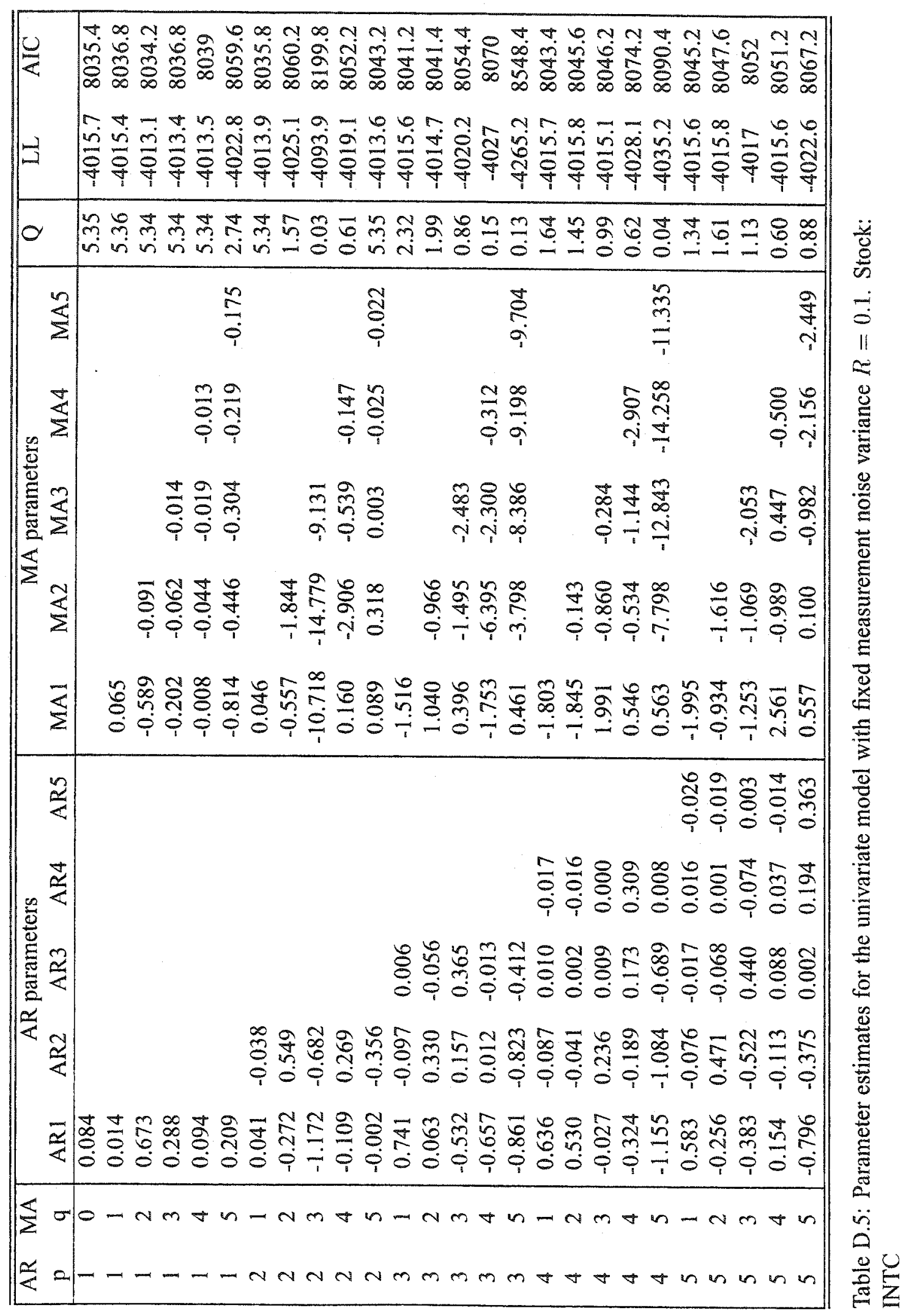




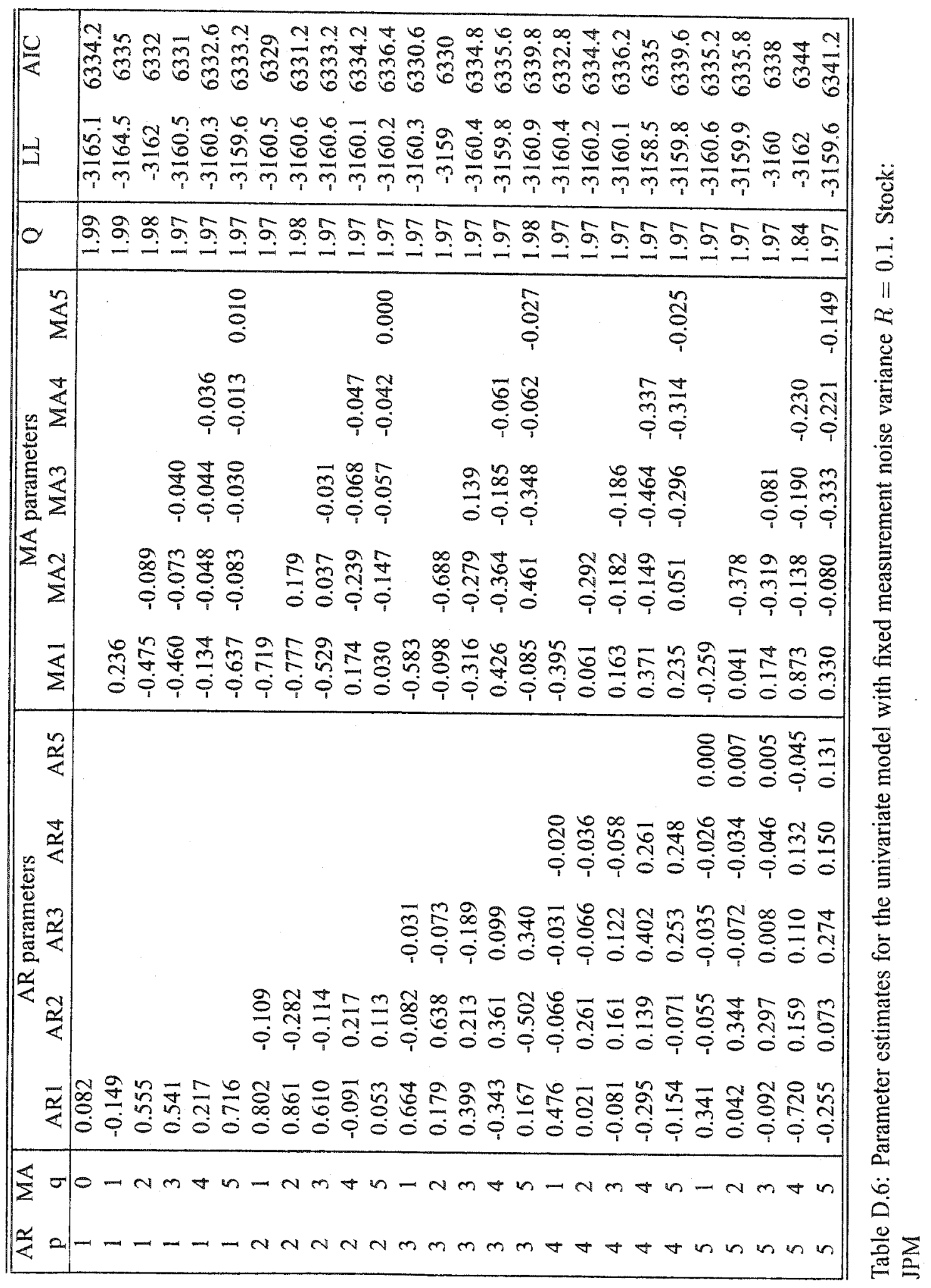




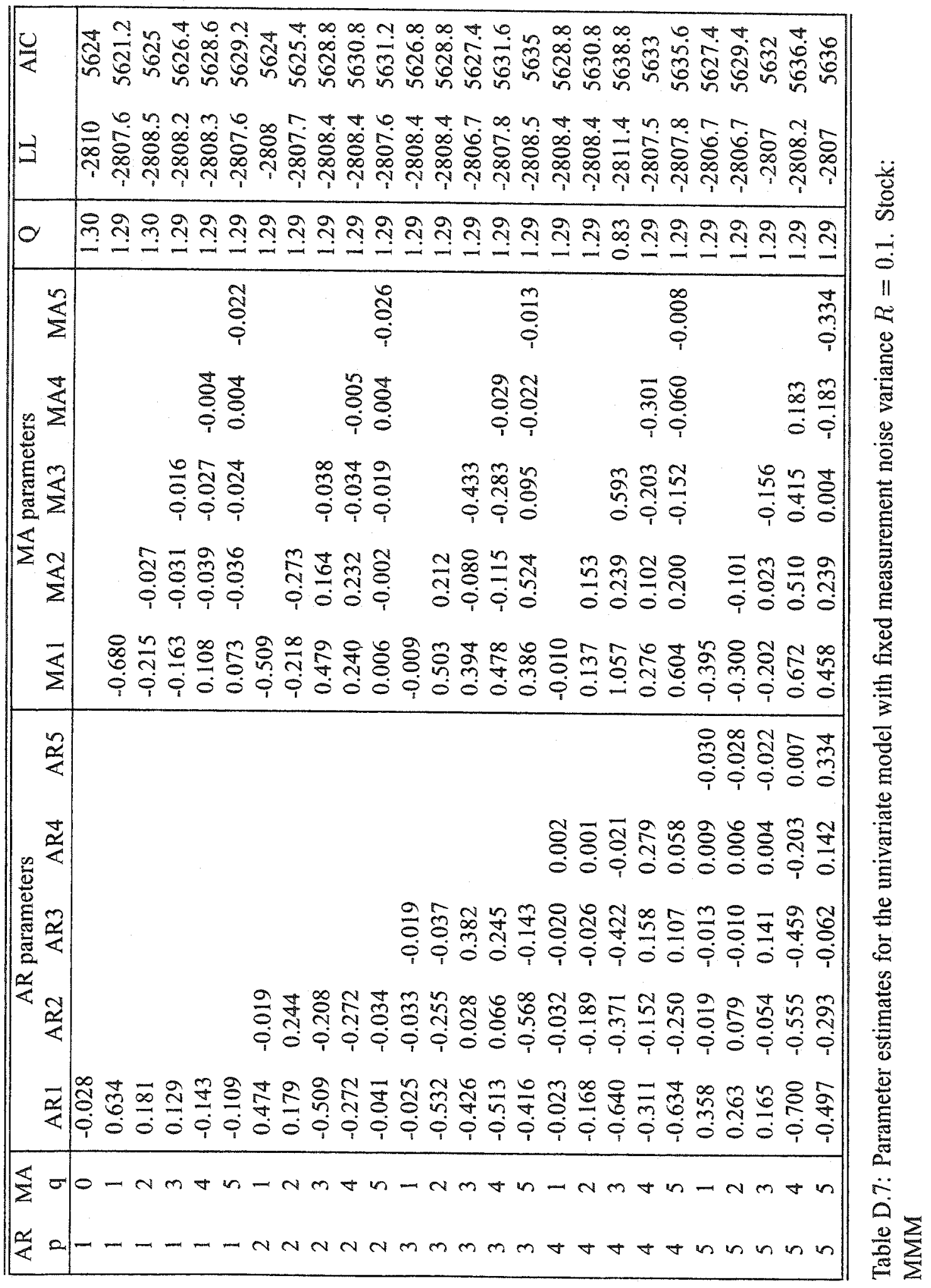




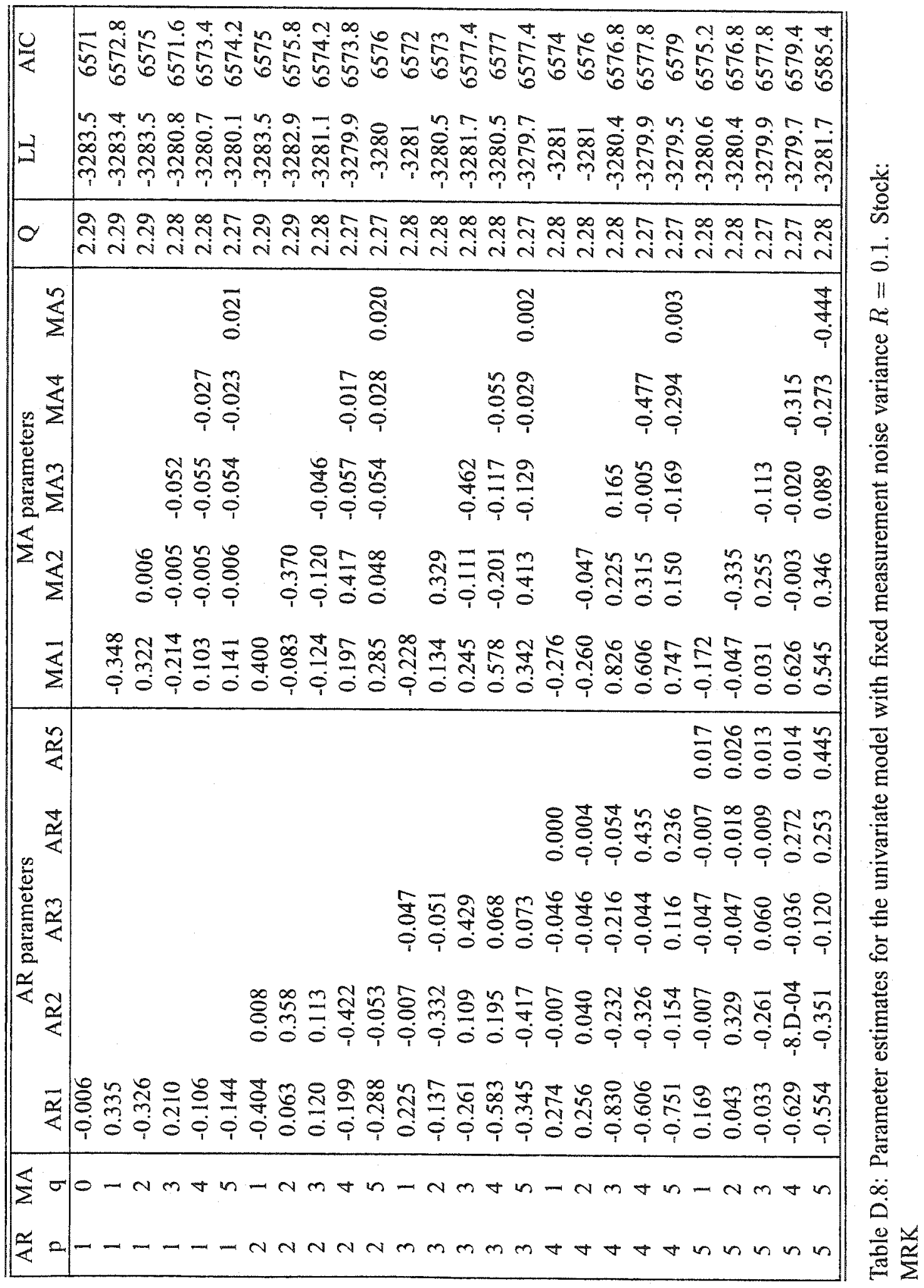




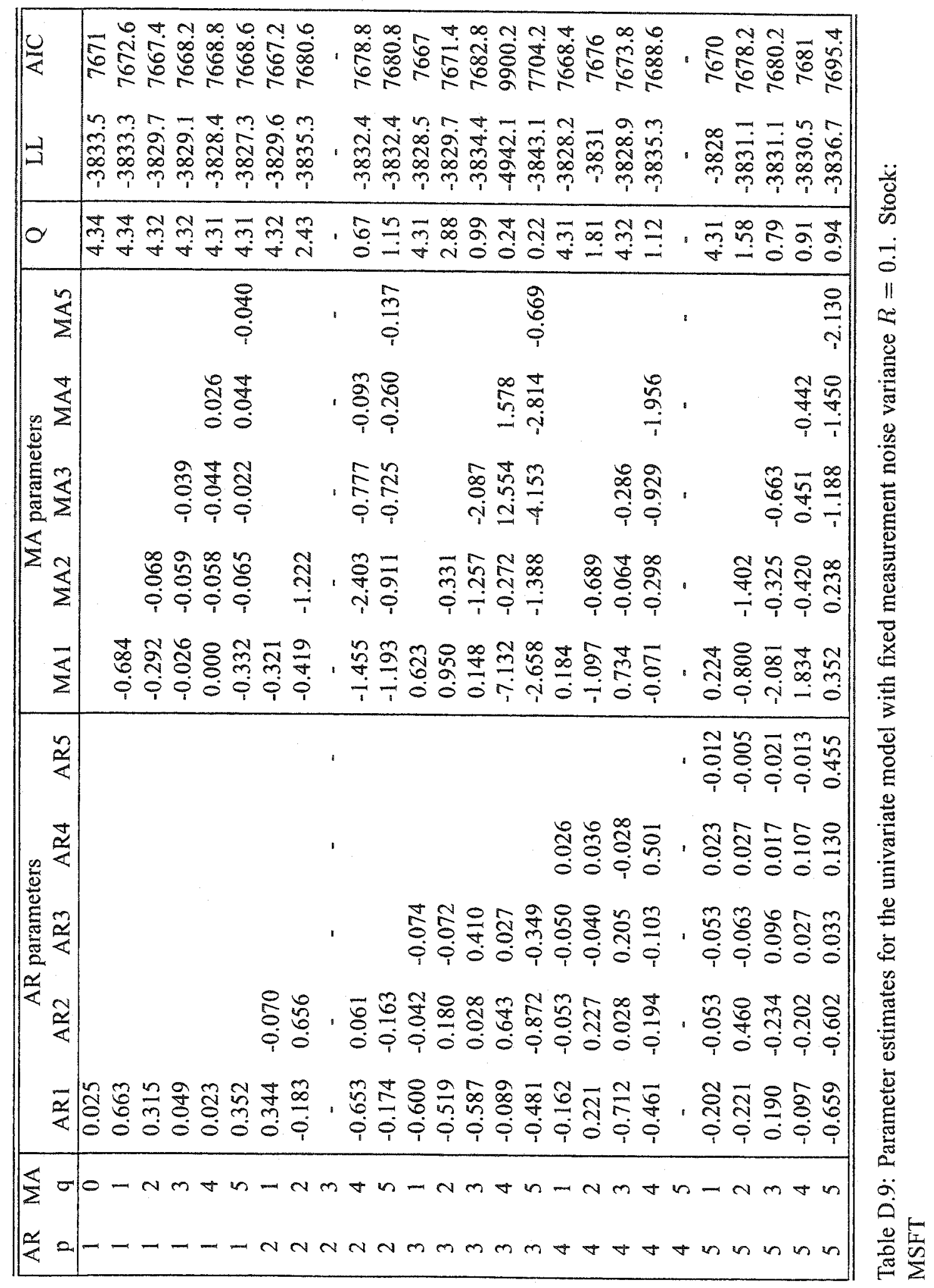




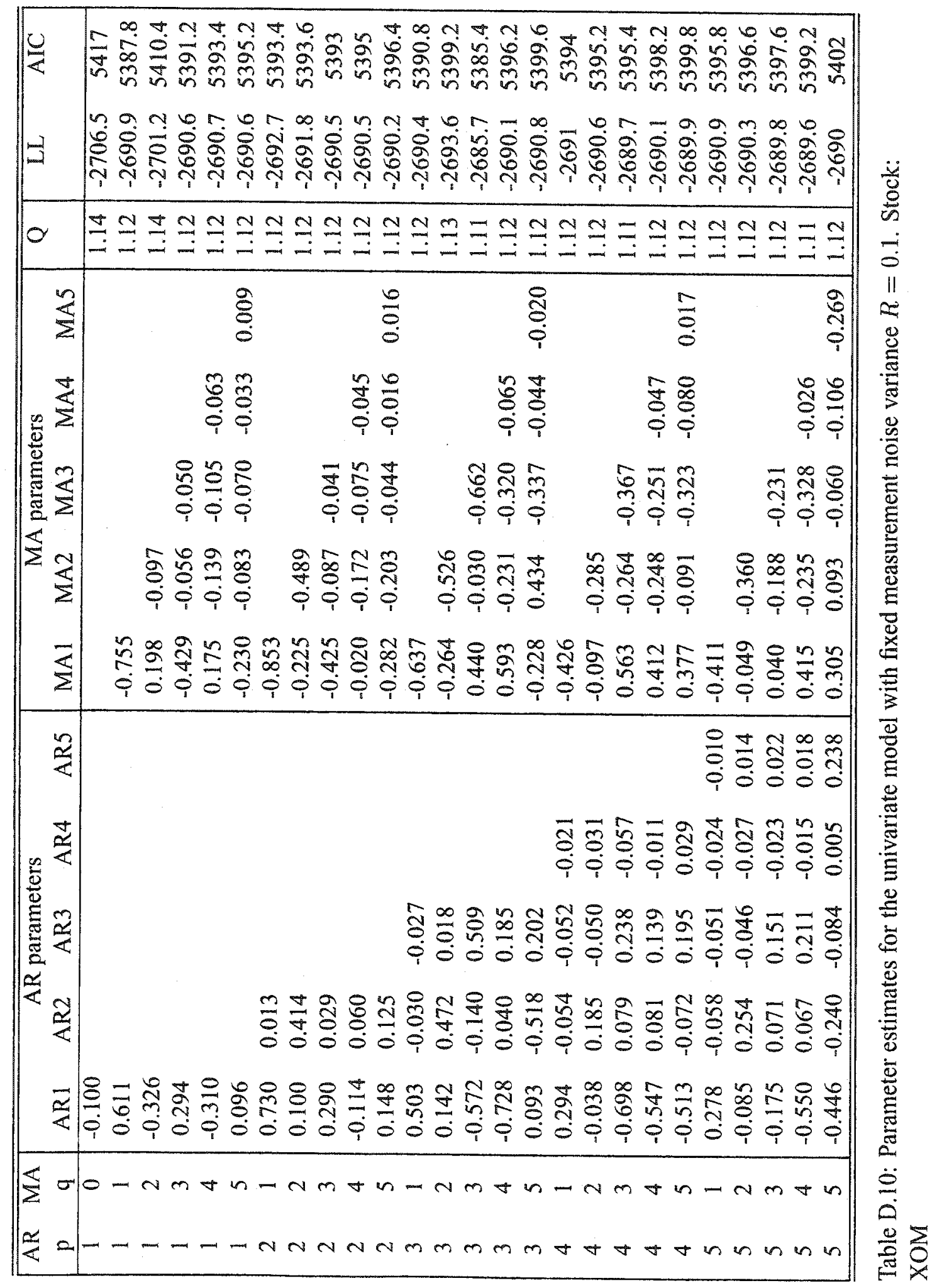




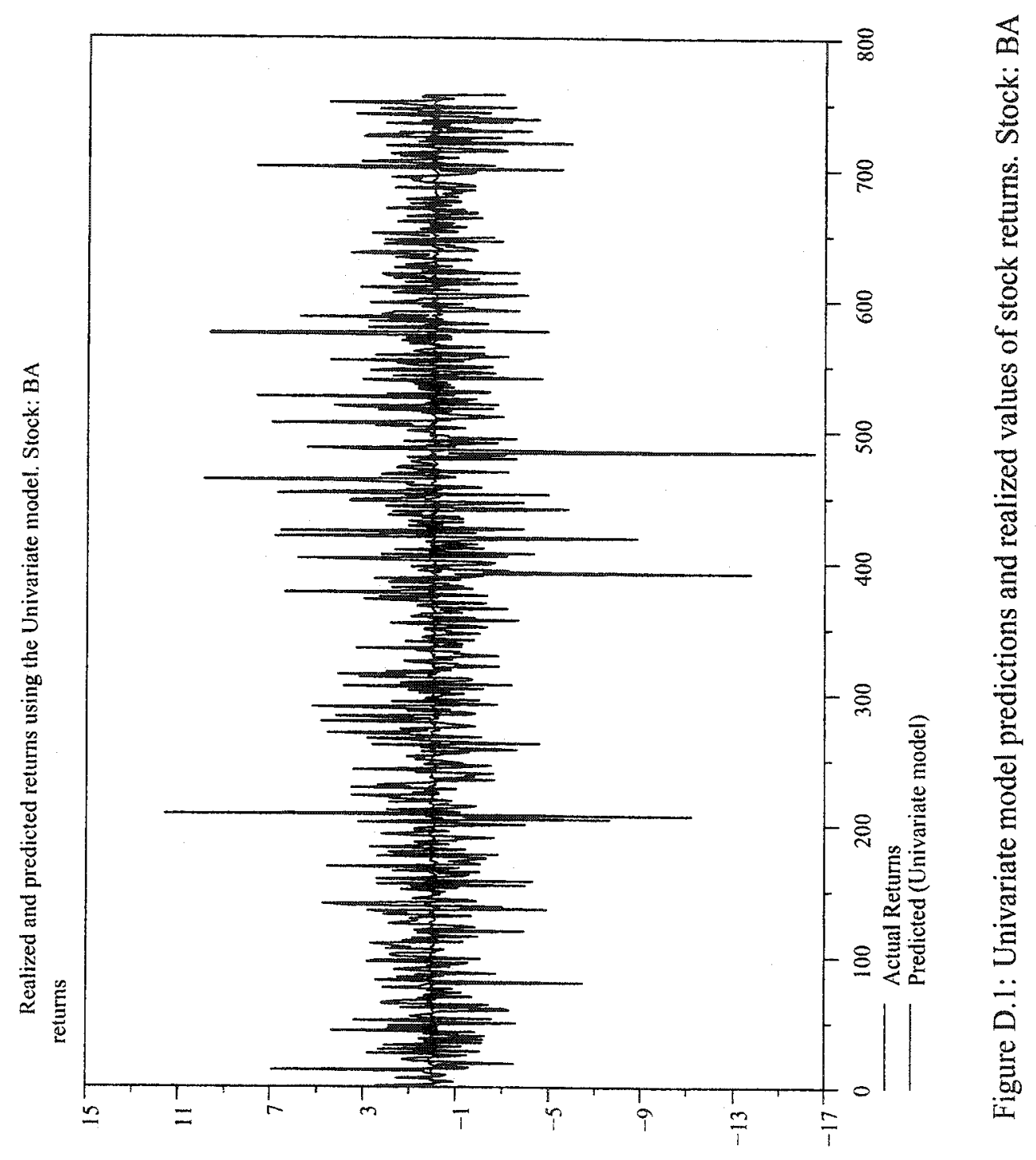




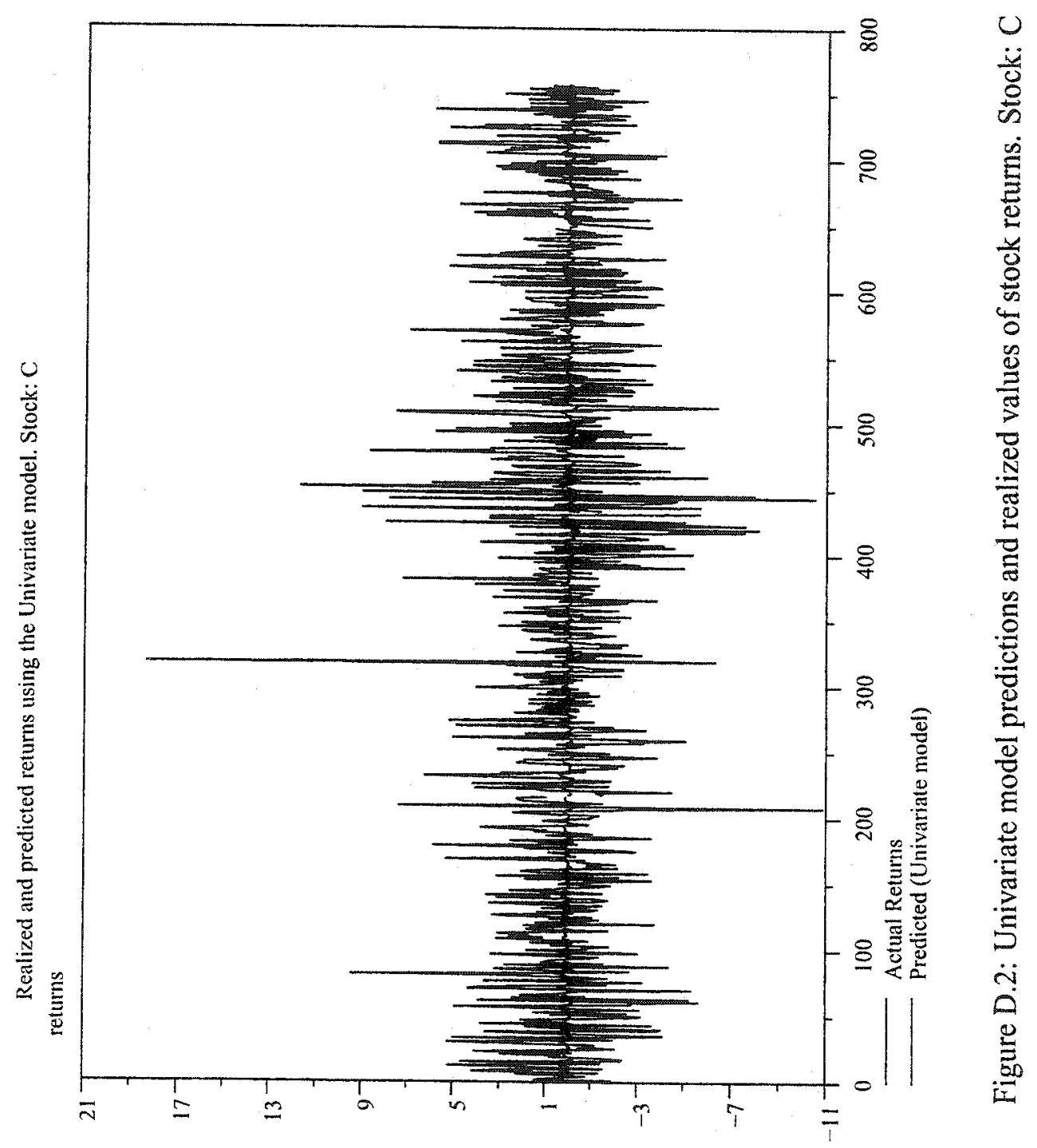




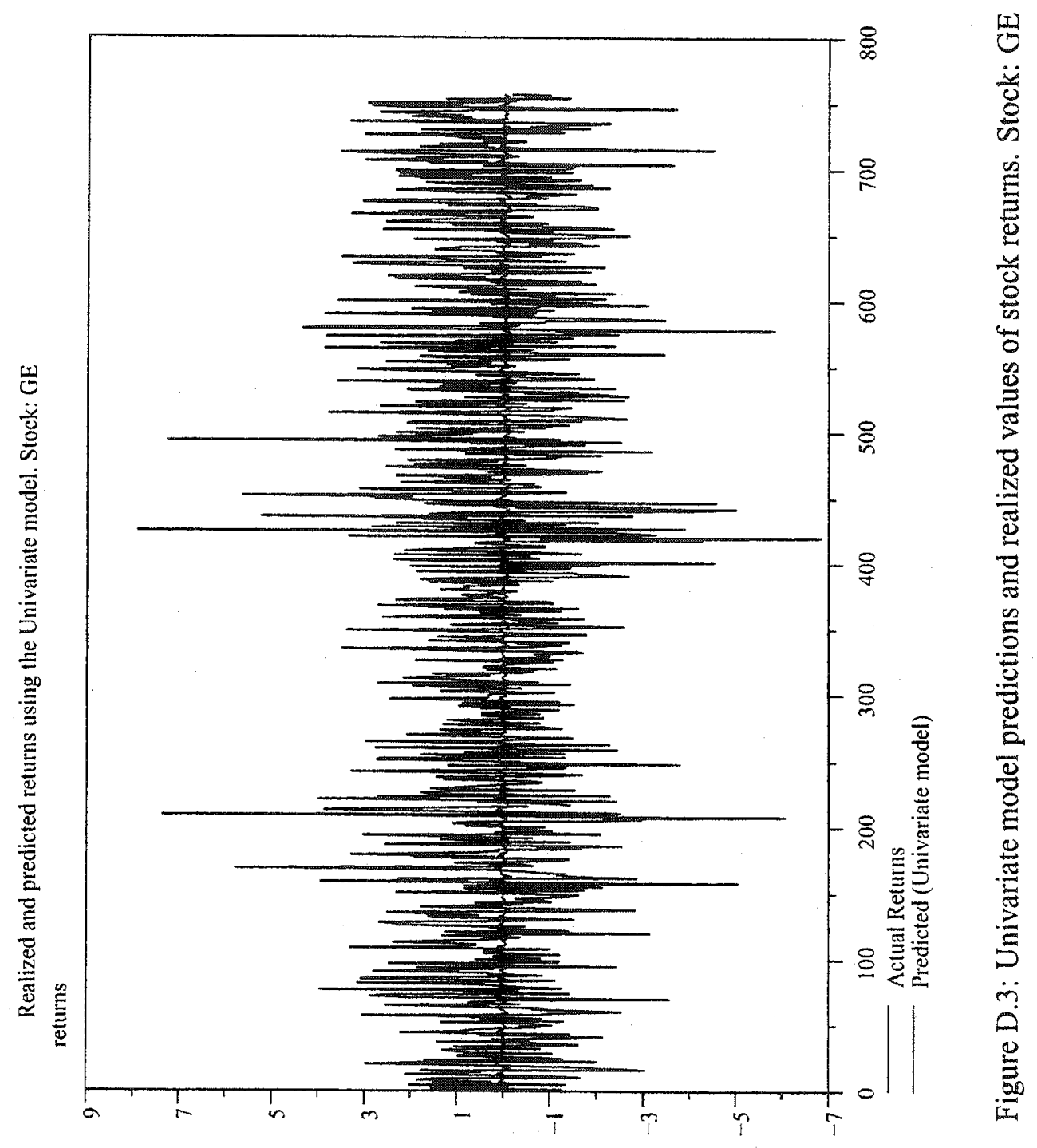




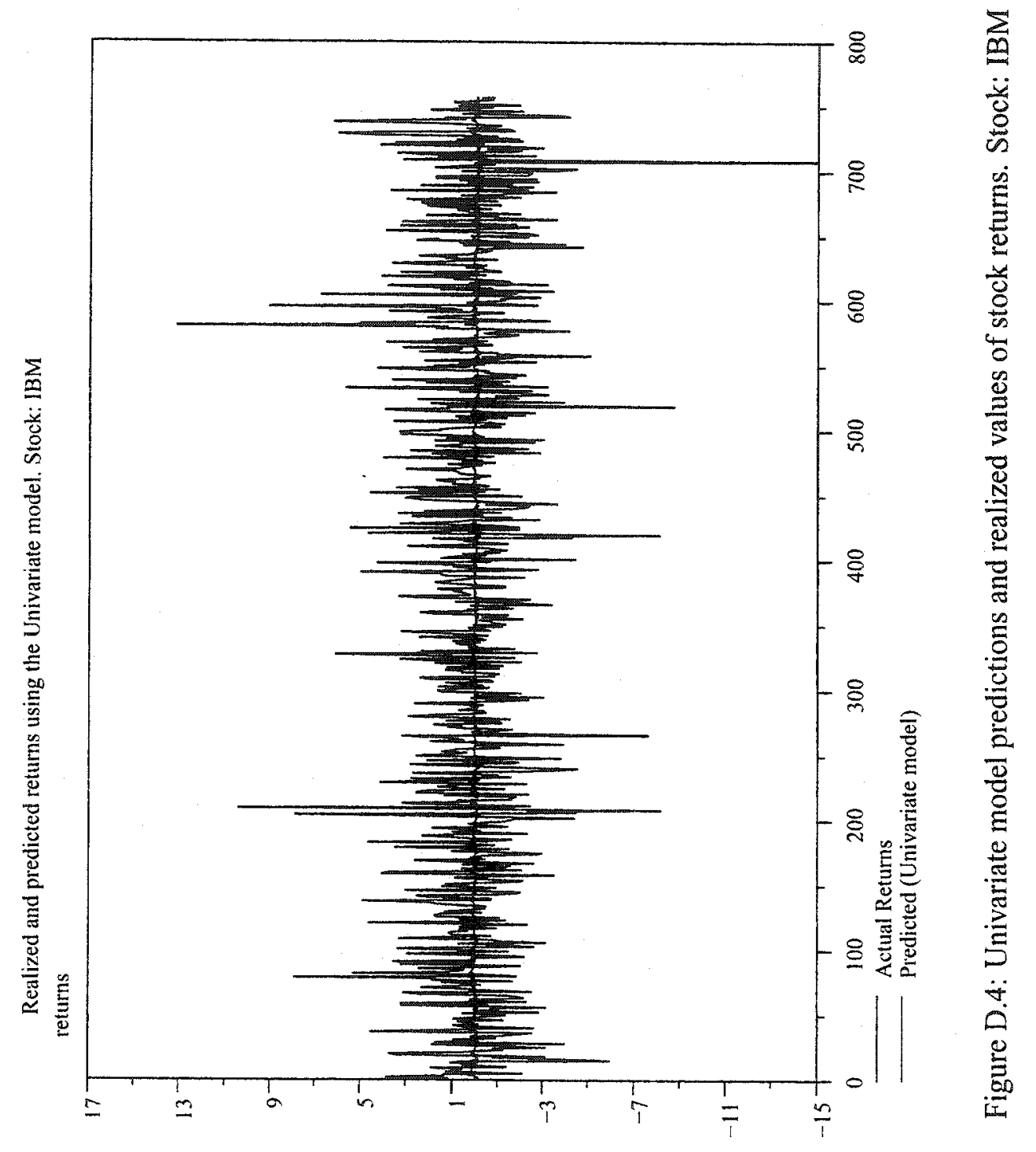




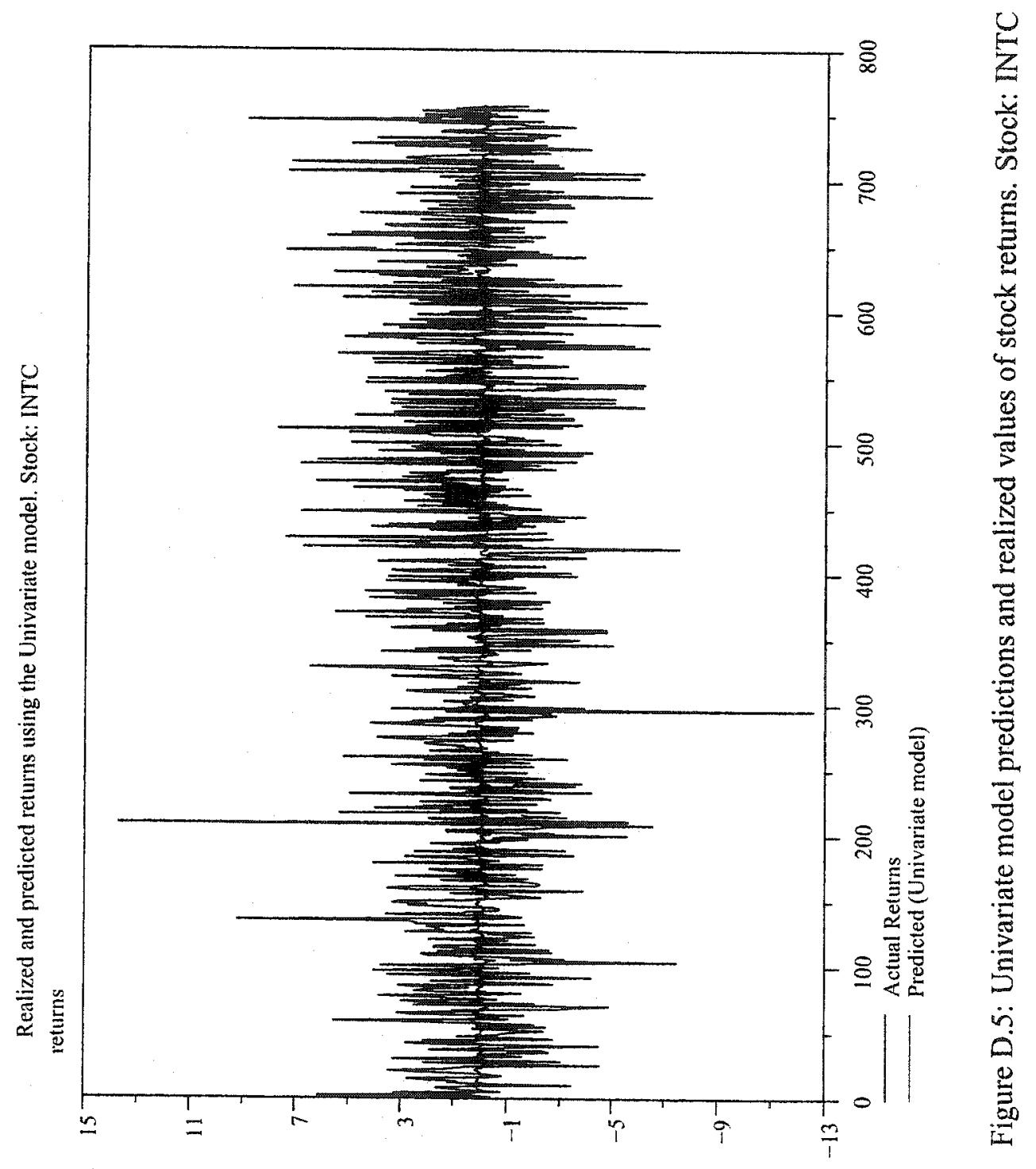




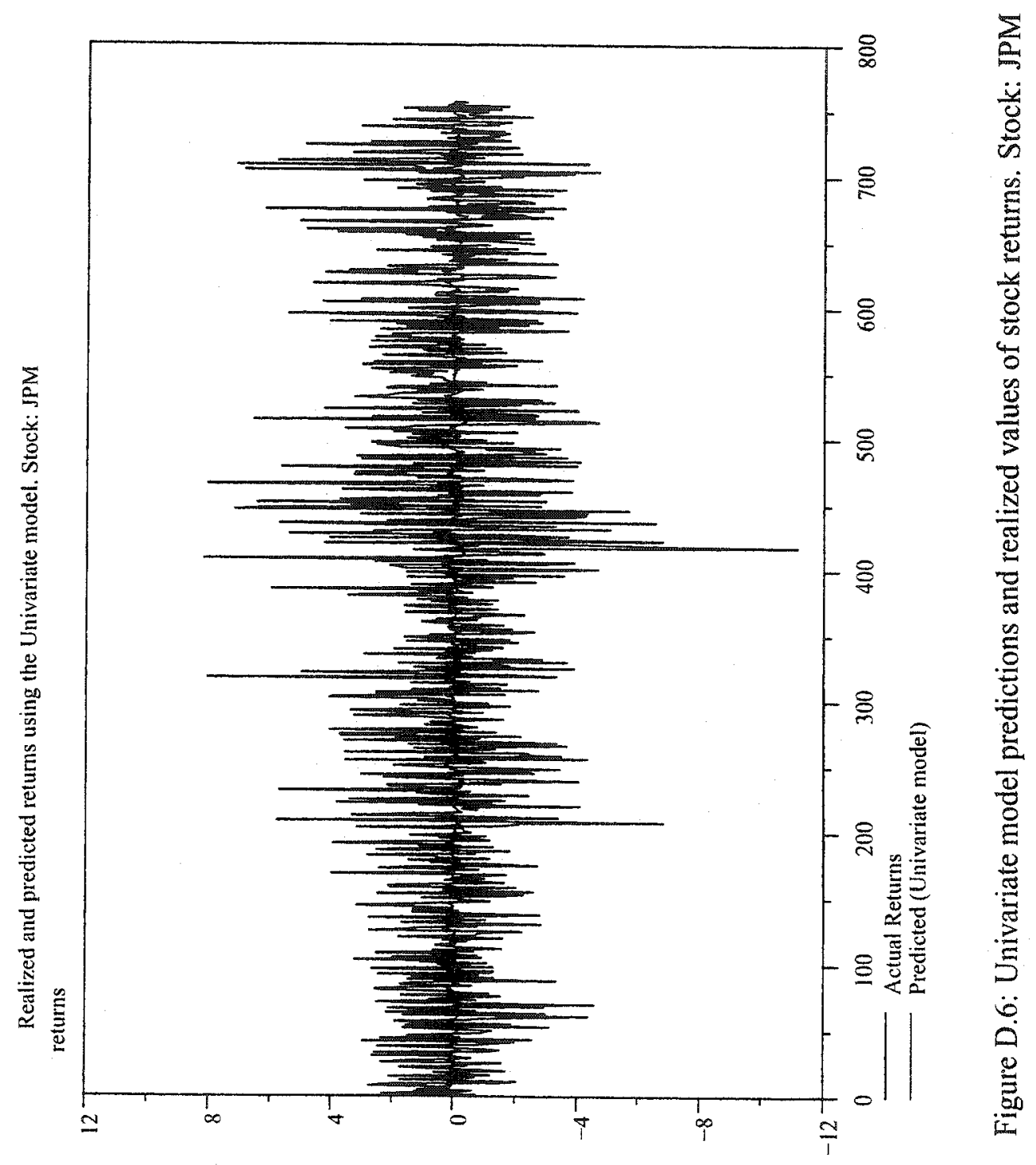




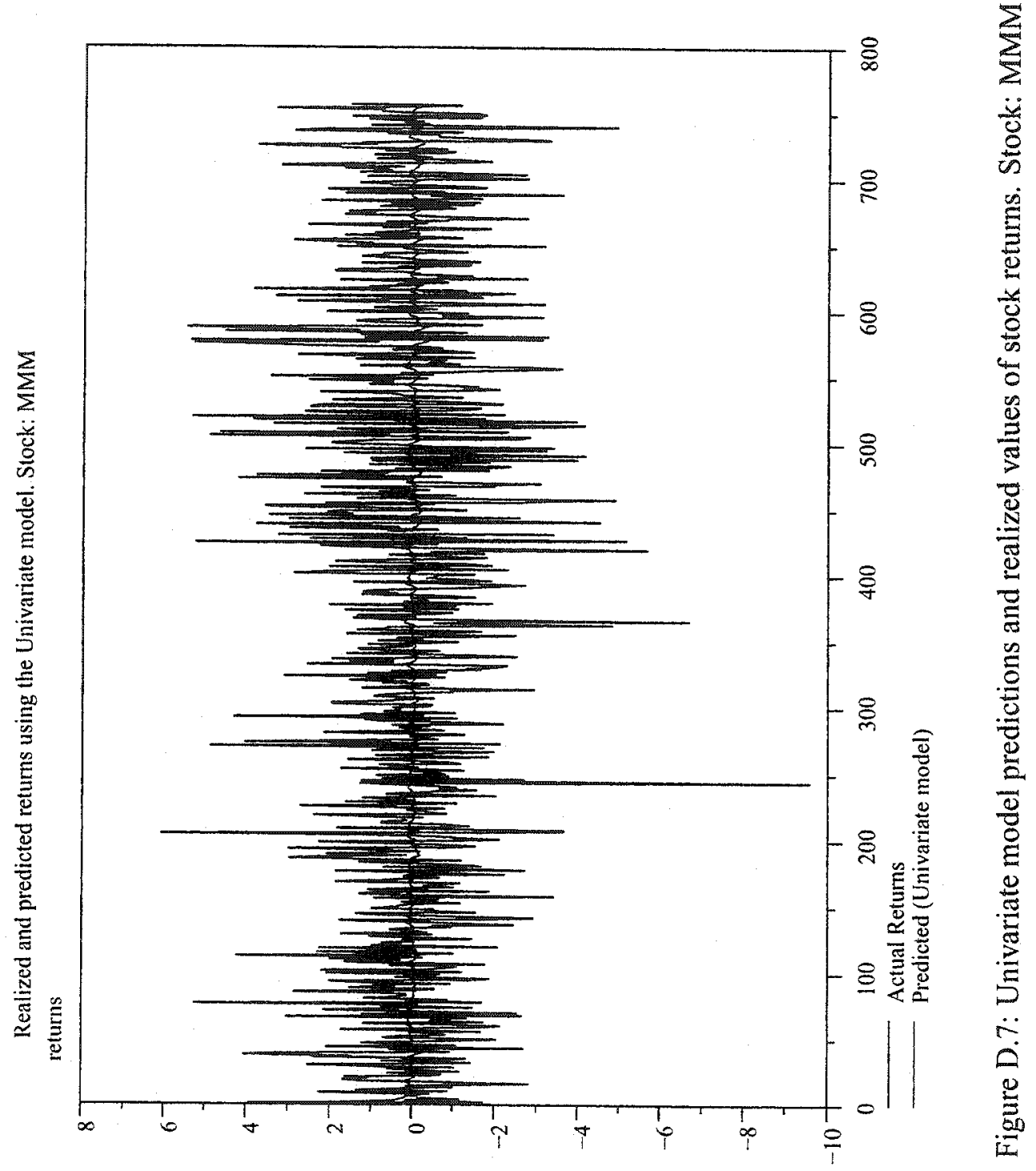




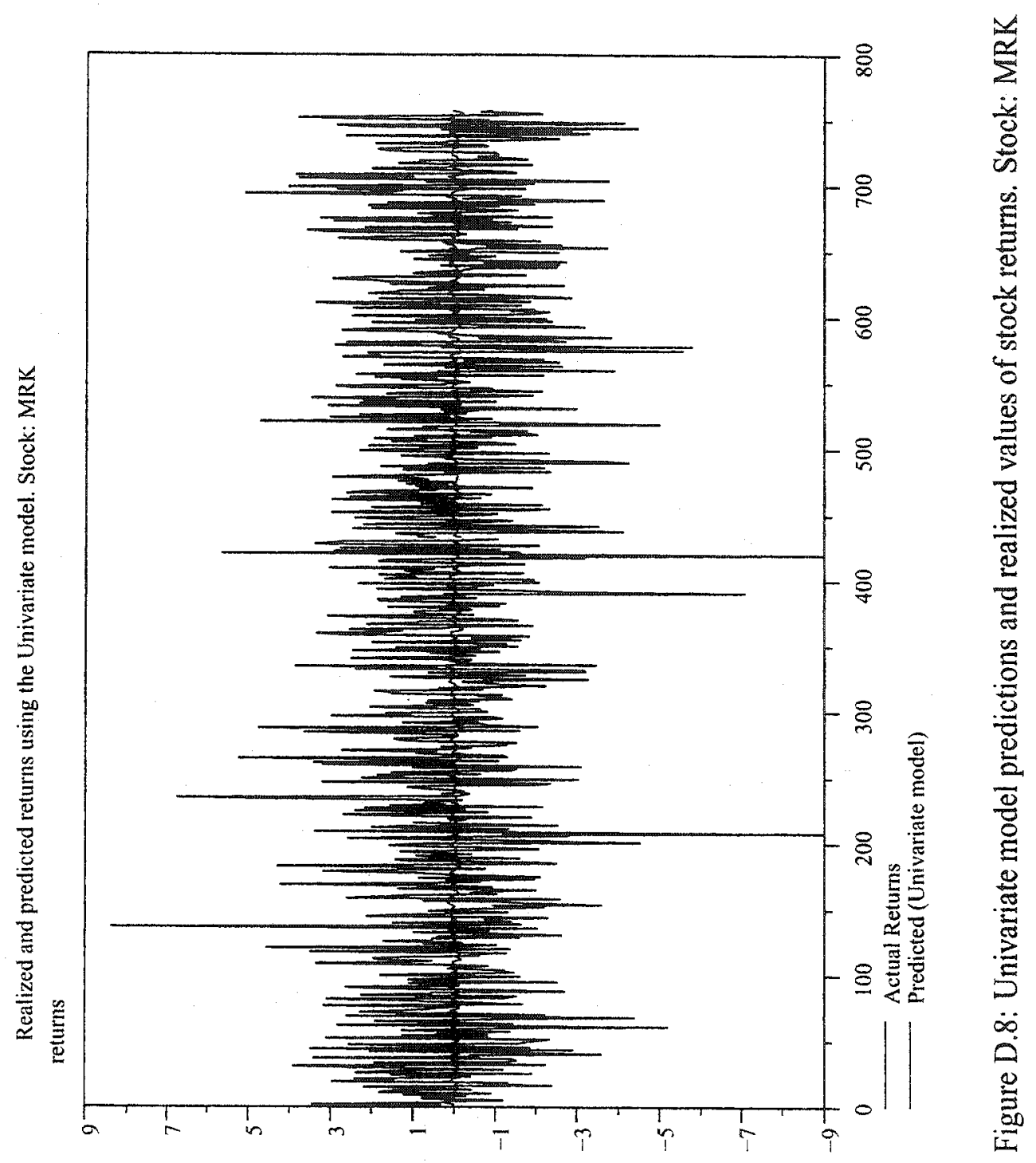




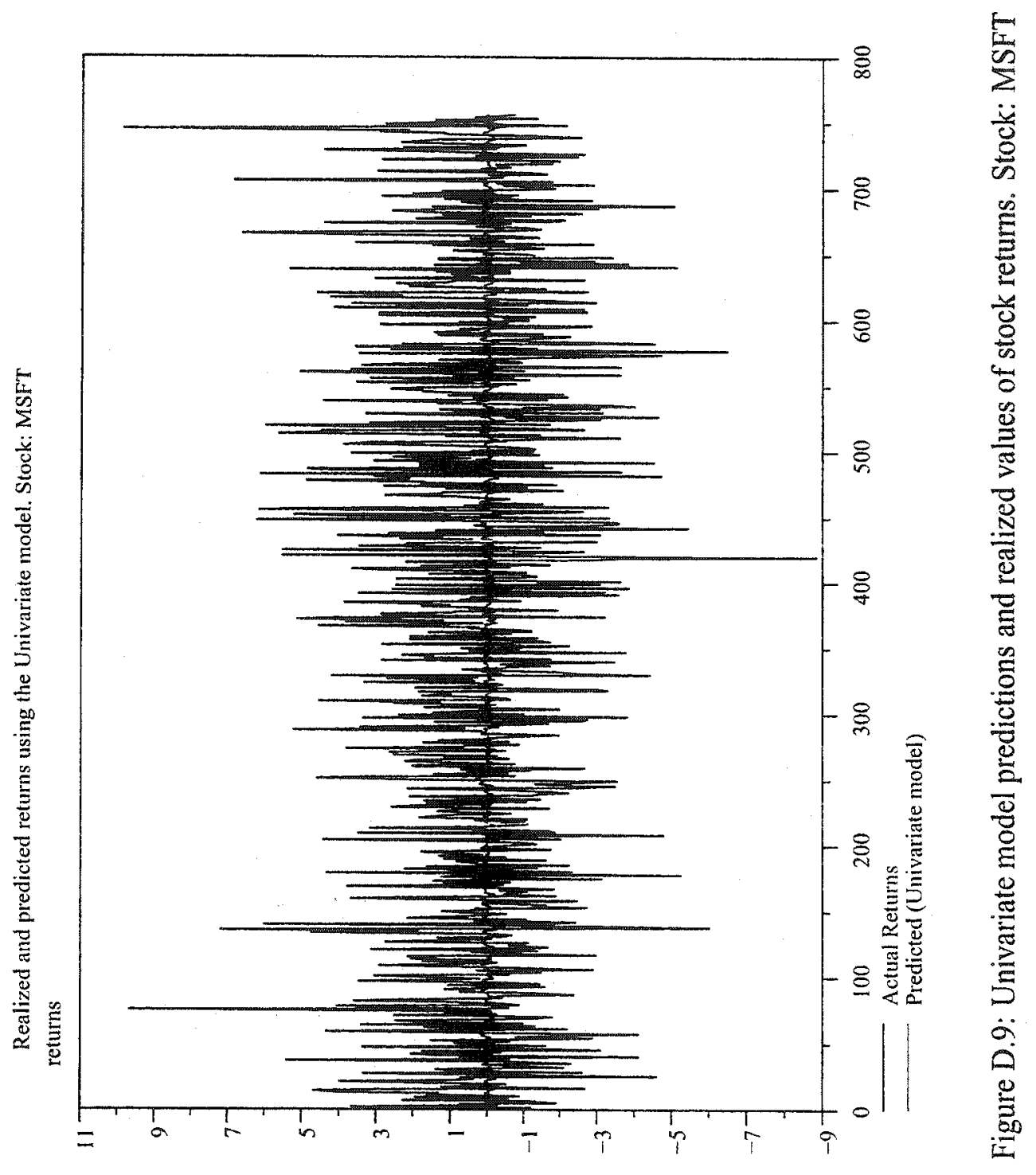




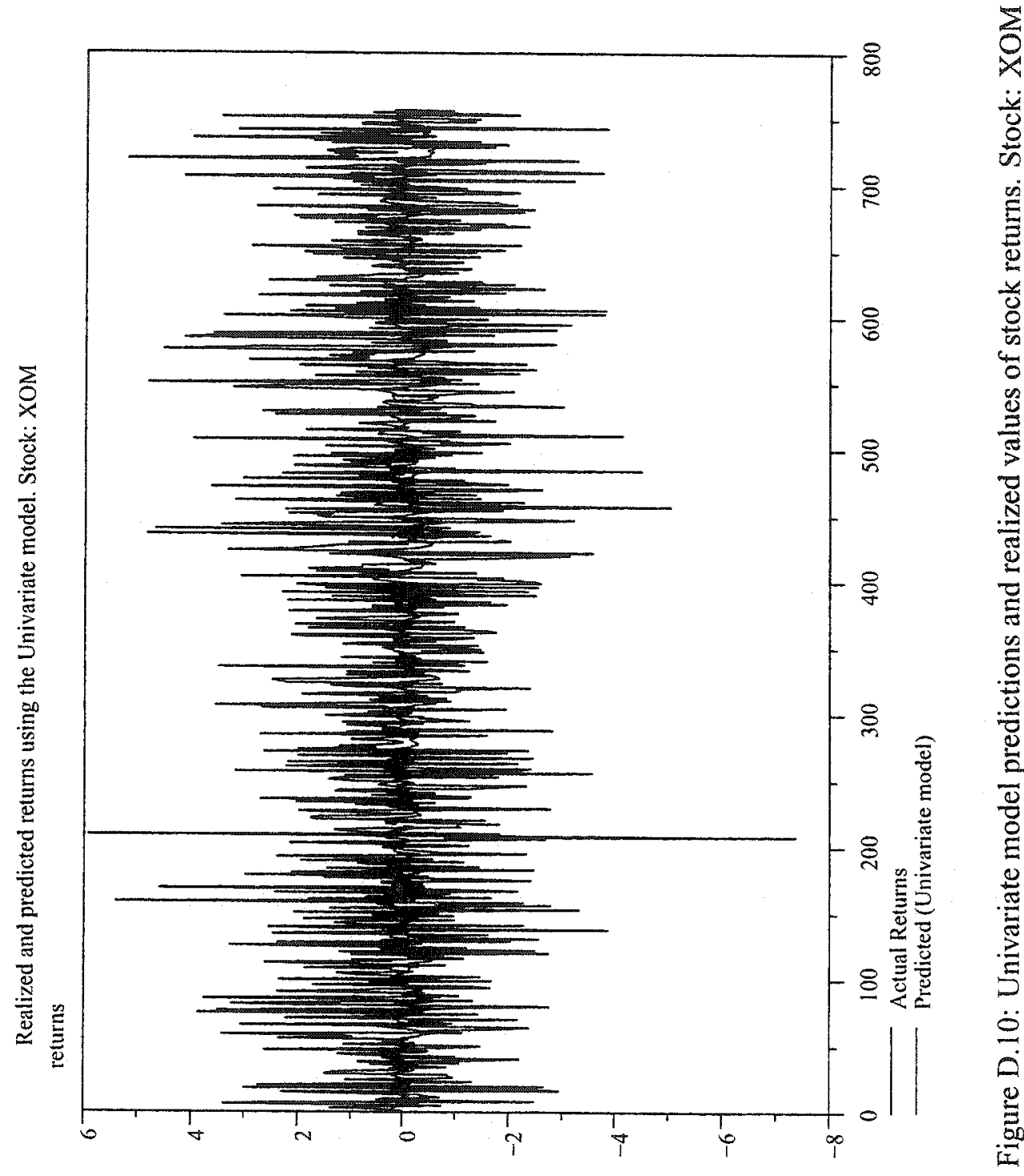




\section{Appendix E}

\section{Multivariate Model}

\section{E.1 Multivariate Model: Market Submodel Estimate}

The market sub-model explains the behaviour of the observed market variables as an AR(1) model. The measurement noise covariance matrix $R$ was assumed to be fixed for tractability purposes. The measurement and state equations with the estimate of the transition matrix $F$ are presented, as well as the estimate of the driving noise covariance matrix $Q$. 


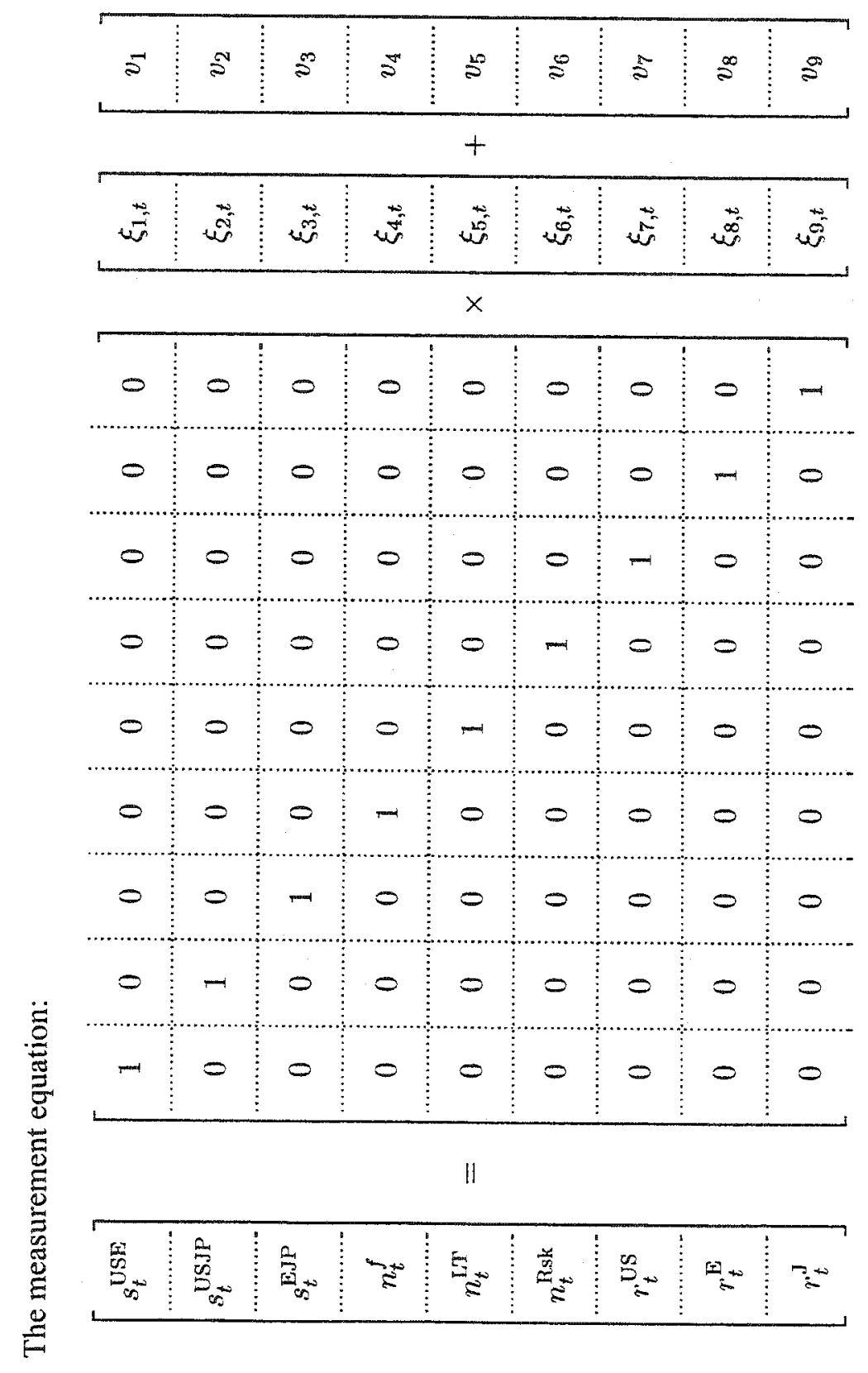




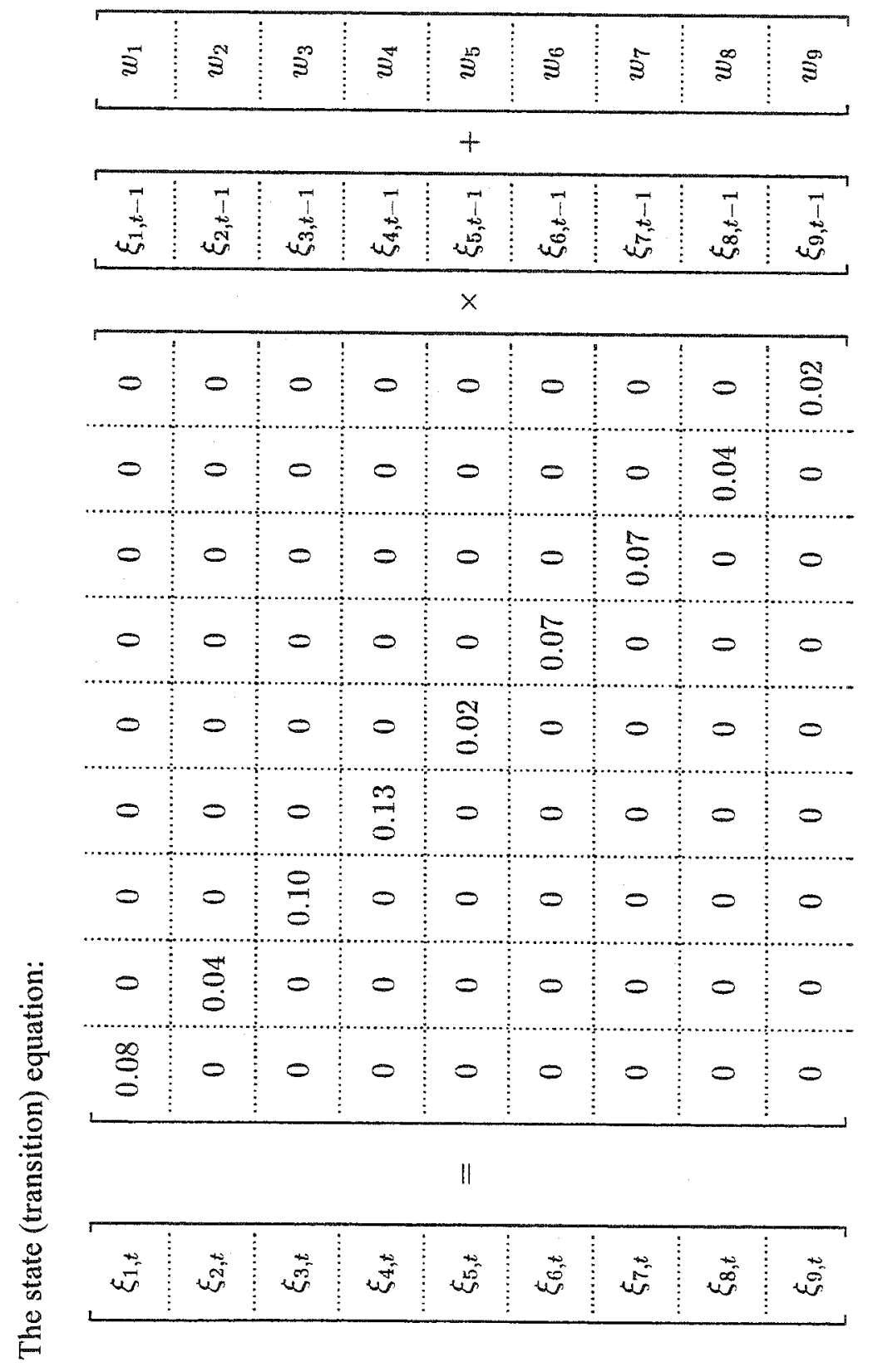




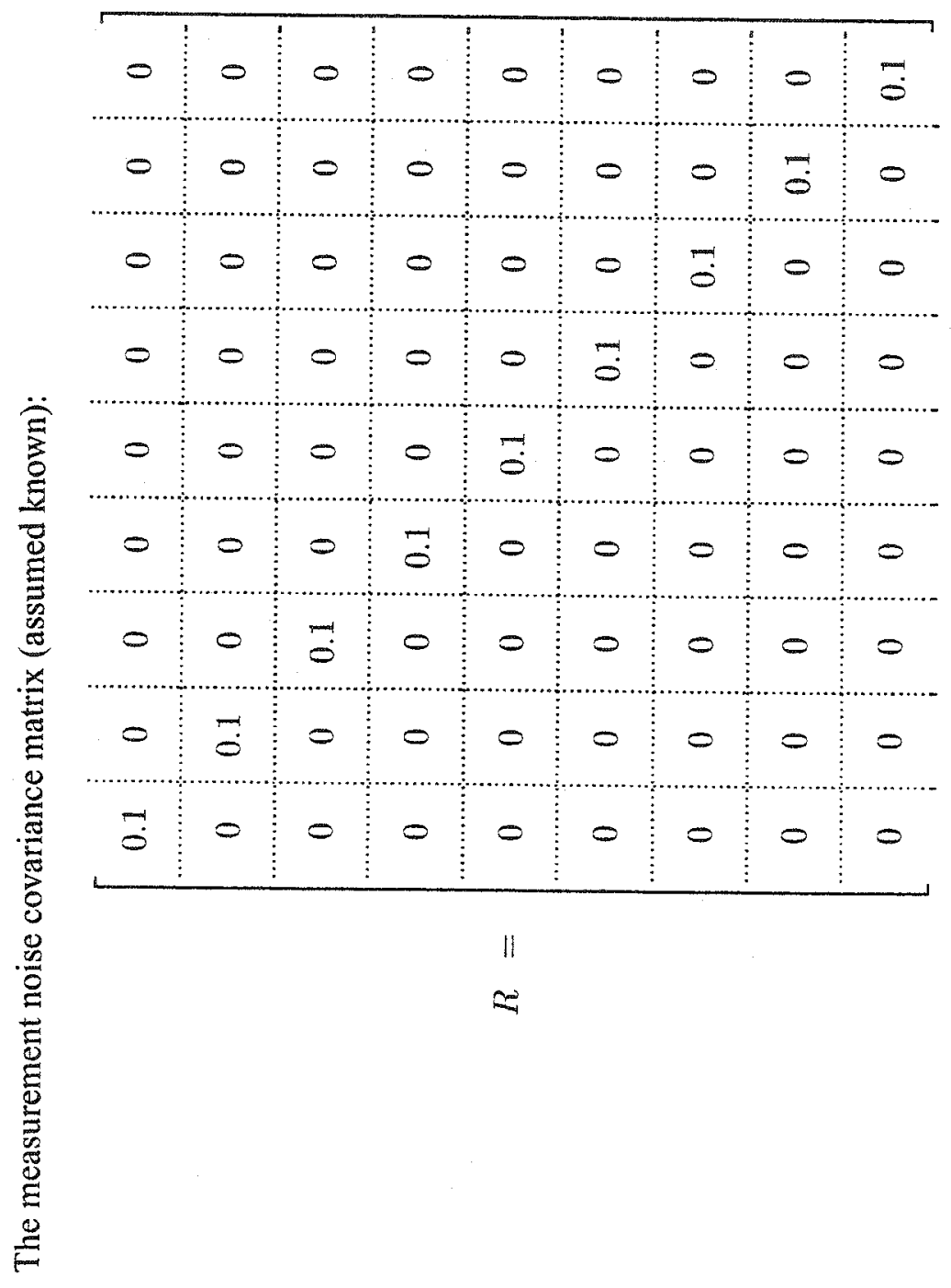




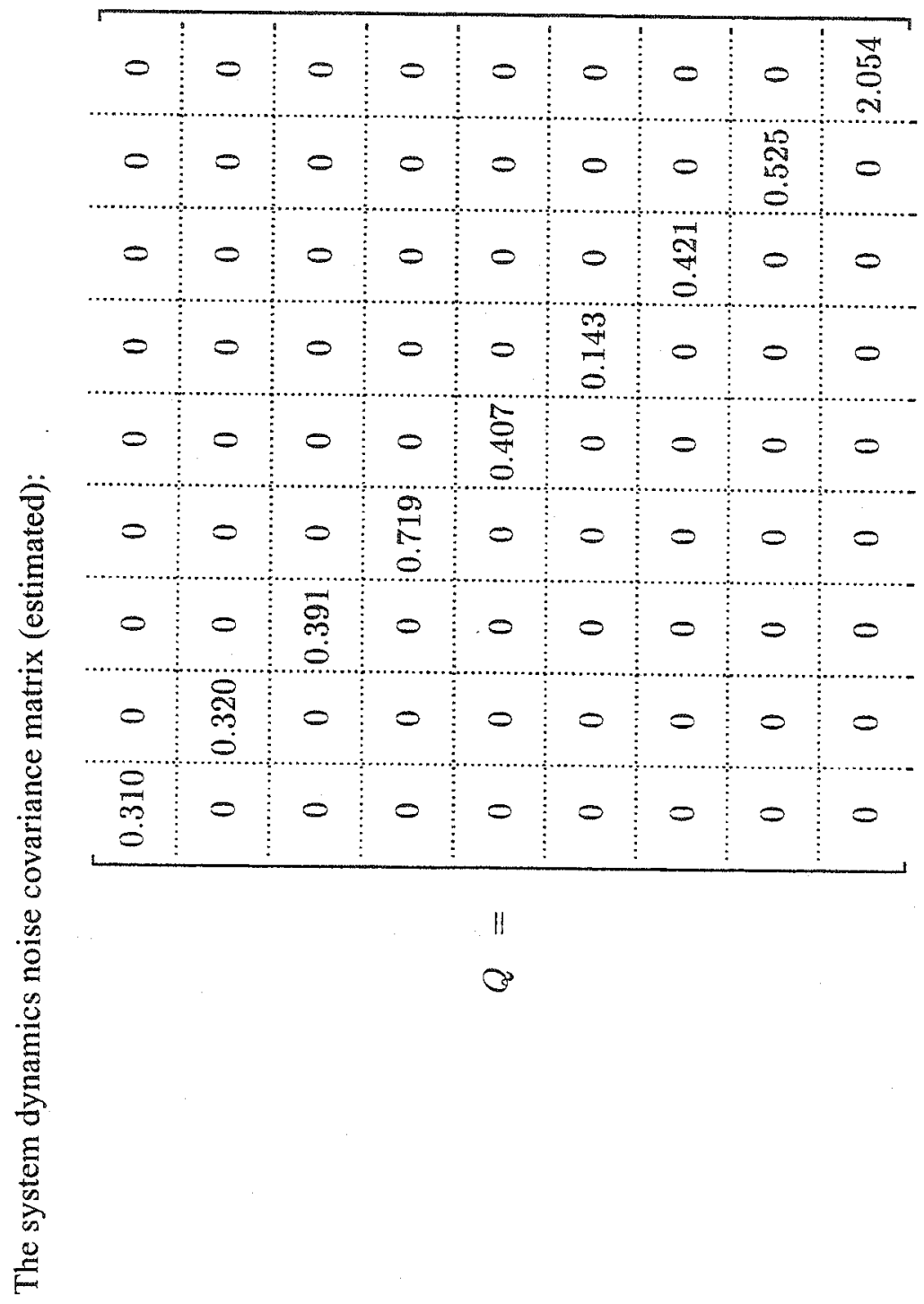




\section{E.2 Multivariate Model: Parameter Estimates}

\begin{tabular}{|c|c|c|c|c|c|c|c|c|c|}
\hline \multirow{2}{*}{$\begin{array}{l}\text { Ticker } \\
\text { Symbol } \\
\mathrm{BA}\end{array}$} & \multirow{2}{*}{$\begin{array}{c}\mathrm{AR} \\
\mathrm{p}\end{array}$} & \multirow{2}{*}{$\begin{array}{c}\mathrm{MA} \\
\mathrm{q}\end{array}$} & \multicolumn{2}{|c|}{ AR parameters } & \multicolumn{5}{|c|}{ MA parameters } \\
\hline & & & ARI & AR2 & MA1 & MA2 & MA3 & MA4 & $Q$ \\
\hline & & 4 & -0.29 & -0.40 & 0.30 & 0.35 & -0.04 & -0.04 & 2.35 \\
\hline & \multicolumn{9}{|c|}{ B2 coefficients } \\
\hline & B2-1 & B2-2 & B2-3 & B2-4 & $B 2-5$ & B2-6 & B2-7 & B2-8 & B2-9 \\
\hline & 5.1 & 10.5 & -4.6 & -0.4 & -26.0 & 12.0 & 1.4 & 2.3 & -1.6 \\
\hline \multirow[t]{5}{*}{$\mathrm{C}$} & $\mathrm{p}$ & q & $\mathrm{AR} 1$ & $\mathrm{AR} 2$ & $\overline{\mathrm{MAl}}$ & $\overline{\mathrm{MA2}}$ & MA3 & $\overline{\mathrm{MA4}}$ & $Q$ \\
\hline & 2 & 1 & 0.63 & -0.05 & -0.60 & & & & 3.79 \\
\hline & \multicolumn{9}{|c|}{ B2 coefficients } \\
\hline & B2-1 & B2-2 & B2-3 & B2-4 & $\mathrm{B} 2-5$ & B2-6 & B2-7 & B2-8 & B2-9 \\
\hline & 150.9 & 295.5 & -112.6 & 0.1 & 6.3 & -6.5 & 2.5 & 3.7 & -0.2 \\
\hline \multirow[t]{5}{*}{$\mathrm{GE}$} & $\mathrm{p}$ & $q$ & $\mathrm{AR} 1$ & AR2 & MA1 & MA2 & $\overline{\mathrm{MA3}}$ & $\overline{\mathrm{MA} 4}$ & $Q$ \\
\hline & 2 & 1 & 0.48 & -0.05 & -0.46 & & & & 1.42 \\
\hline & \multicolumn{9}{|c|}{ B2 coefficients } \\
\hline & B2-1 & B2-2 & B2-3 & B2-4 & B2-5 & B2-6 & B2-7 & B2-8 & B2-9 \\
\hline & 72.9 & 143.4 & -54.5 & -0.2 & 3.4 & -1.8 & 0.1 & 3.1 & -1.8 \\
\hline \multirow[t]{5}{*}{ IBM } & $\mathrm{p}$ & $q$ & AR1 & $\overline{A R 2}$ & $\overline{\mathrm{MA1}}$ & $\overline{\mathrm{MA2}}$ & $\overline{\mathrm{MA3}}$ & MA4 & $Q$ \\
\hline & 2 & 2 & -0.02 & 0.56 & 0.07 & -0.57 & & & 2.85 \\
\hline & \multicolumn{9}{|c|}{ B2 coefficients } \\
\hline & B2-1 & B2-2 & B2-3 & B2-4 & B2-5 & $B 2-6$ & B2-7 & B2-8 & $B 2-9$ \\
\hline & 94.8 & 187.3 & -72.4 & -0.3 & 9.8 & -2.3 & -1.3 & 4.1 & -0.2 \\
\hline \multirow[t]{5}{*}{ INTC } & $\mathrm{p}$ & $q$ & AR1 & AR2 & $\overline{\mathrm{MA1}}$ & MA2 & MA3 & MA4 & $\bar{Q}$ \\
\hline & 1 & 2 & 0.74 & & -0.68 & -0.06 & & & 5.69 \\
\hline & \multicolumn{9}{|c|}{ B2 coefficients } \\
\hline & B2-1 & B2-2 & $B 2-3$ & B2-4 & B2-5 & B2-6 & B2-7 & B2-8 & B2-9 \\
\hline & 169.0 & 329.2 & -127.0 & 0.9 & -8.2 & 2.5 & -1.0 & -0.5 & -1.6 \\
\hline
\end{tabular}

Table E.1: Multivariate model parameters. Stocks BA - INTC.

Measurement error variance assumed to be $R=0.1$. 


\begin{tabular}{|c|c|c|c|c|c|c|c|c|c|}
\hline \multirow{2}{*}{$\begin{array}{l}\text { Ticker } \\
\text { Symbol } \\
\text { JPM }\end{array}$} & \multirow{2}{*}{$\frac{\mathrm{AR}}{\mathrm{p}}$} & \multirow{2}{*}{$\frac{\text { MA }}{q}$} & \multicolumn{2}{|c|}{ AR parameters } & \multicolumn{4}{|c|}{ MA parameters } & \multirow{3}{*}{$\begin{array}{c}Q \\
1.95\end{array}$} \\
\hline & & & ARI & $\mathrm{AR2}$ & MA1 & $\mathrm{MA2}$ & MA3 & MA4 & \\
\hline & 1 & 3 & 0.49 & & -0.42 & -0.07 & -0.04 & & \\
\hline & \multicolumn{9}{|c|}{ B2 coefficients } \\
\hline & B2-1 & B2-2 & B2-3 & B2-4 & B2-5 & $\mathrm{B} 2-6$ & B2-7 & B2-8 & B2-9 \\
\hline & 82.5 & 158.7 & -60.6 & -0.9 & -13.5 & 1.8 & -1.3 & 1.7 & 0.2 \\
\hline \multirow[t]{5}{*}{$\mathrm{MMM}$} & $\mathrm{p}$ & $\mathrm{q}$ & $\overline{\mathrm{AR}} 1$ & AR2 & MAI & MA2 & MA3 & $\overline{\text { MA4 }}$ & $\mathrm{Q}$ \\
\hline & 2 & 2 & 0.08 & 0.30 & -0.18 & -0.33 & & & 1.27 \\
\hline & \multicolumn{9}{|c|}{ B2 coefficients } \\
\hline & B2-1 & $\mathrm{B} 2-2$ & B2-3 & B2-4 & B2-5 & $\mathrm{B} 2-6$ & $\mathrm{~B} 2-7$ & $\mathrm{~B} 2-8$ & B2-9 \\
\hline & -7.9 & -13.4 & 5.1 & 0.4 & -0.9 & -1.5 & 2.1 & 3.2 & -1.7 \\
\hline \multirow[t]{5}{*}{ MRK } & $\mathrm{p}$ & $q$ & AR1 & $\overline{A R 2}$ & $\mathrm{MA1}$ & MA2 & MA3 & $\overline{\mathrm{MA} 4}$ & $\mathrm{Q}$ \\
\hline & 1 & 3 & 0.21 & & -0.21 & -0.01 & -0.06 & & 2.26 \\
\hline & \multicolumn{9}{|c|}{ B2 coefficients } \\
\hline & B2-1 & B2-2 & B2-3 & B2-4 & B2-5 & $\mathrm{B} 2-6$ & B2-7 & B2-8 & B2-9 \\
\hline & 6.6 & 16.4 & -7.0 & -0.1 & -8.4 & 2.6 & -1.3 & 3.5 & 0.1 \\
\hline \multirow[t]{5}{*}{ MSFT } & $\mathrm{p}$ & $\mathrm{q}$ & ARI & $\mathrm{AR2}$ & MAI & $\overline{\text { MA2 }}$ & MA3 & MA4 & $\mathrm{Q}$ \\
\hline & 2 & 1 & 0.37 & -0.07 & -0.35 & & & & 4.31 \\
\hline & \multicolumn{9}{|c|}{ B2 coefficients } \\
\hline & B2-1 & B2-2 & B2-3 & B2-4 & B2-5 & B2-6 & B2-7 & $\mathrm{B} 2-8$ & B2-9 \\
\hline & 75.1 & 149.5 & -57.4 & 0.1 & -7.8 & 4.0 & -0.2 & 1.3 & 1.3 \\
\hline \multirow[t]{5}{*}{$\mathrm{XOM}$} & $\mathrm{p}$ & $q$ & ARI & $\overline{\mathrm{AR} 2}$ & MA1 & MA2 & MA3 & MA4 & $\mathrm{Q}$ \\
\hline & 1 & 1 & 0.62 & & -0.77 & & & & 1.11 \\
\hline & \multicolumn{9}{|c|}{ B2 coefficients } \\
\hline & B2-1 & $B 2-2$ & B2-3 & B2-4 & $\mathrm{B} 2-5$ & B2-6 & B2-7 & $\mathrm{B} 2-8$ & B2-9 \\
\hline & 50.0 & 95.7 & -36.8 & 0.3 & -1.6 & -2.5 & 0.0 & 1.7 & 0.7 \\
\hline
\end{tabular}

Table E.2: Multivariate model parameters. Stocks JPM - XOM.

Measurement error variance assumed to be $R=0.1$. 


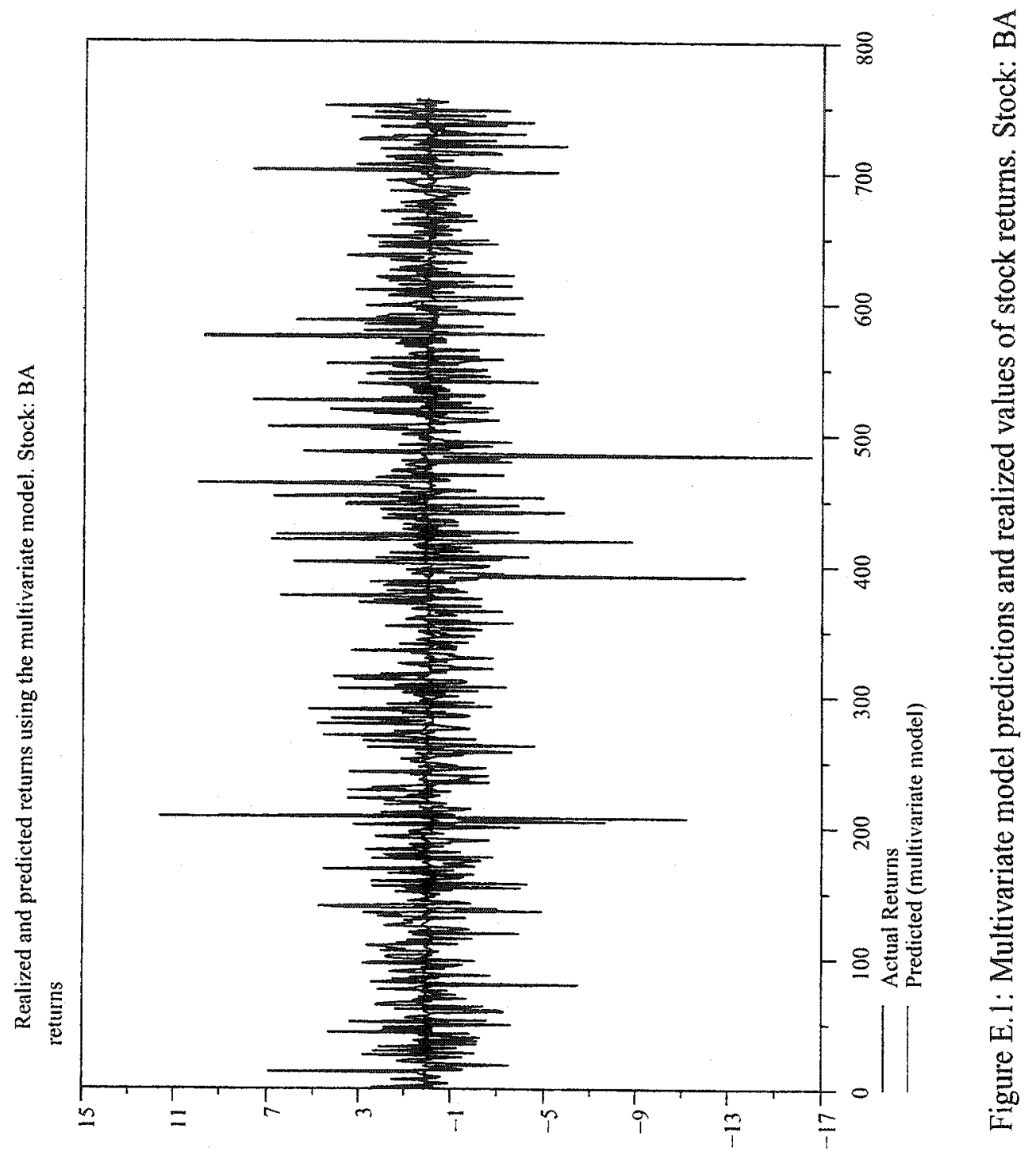




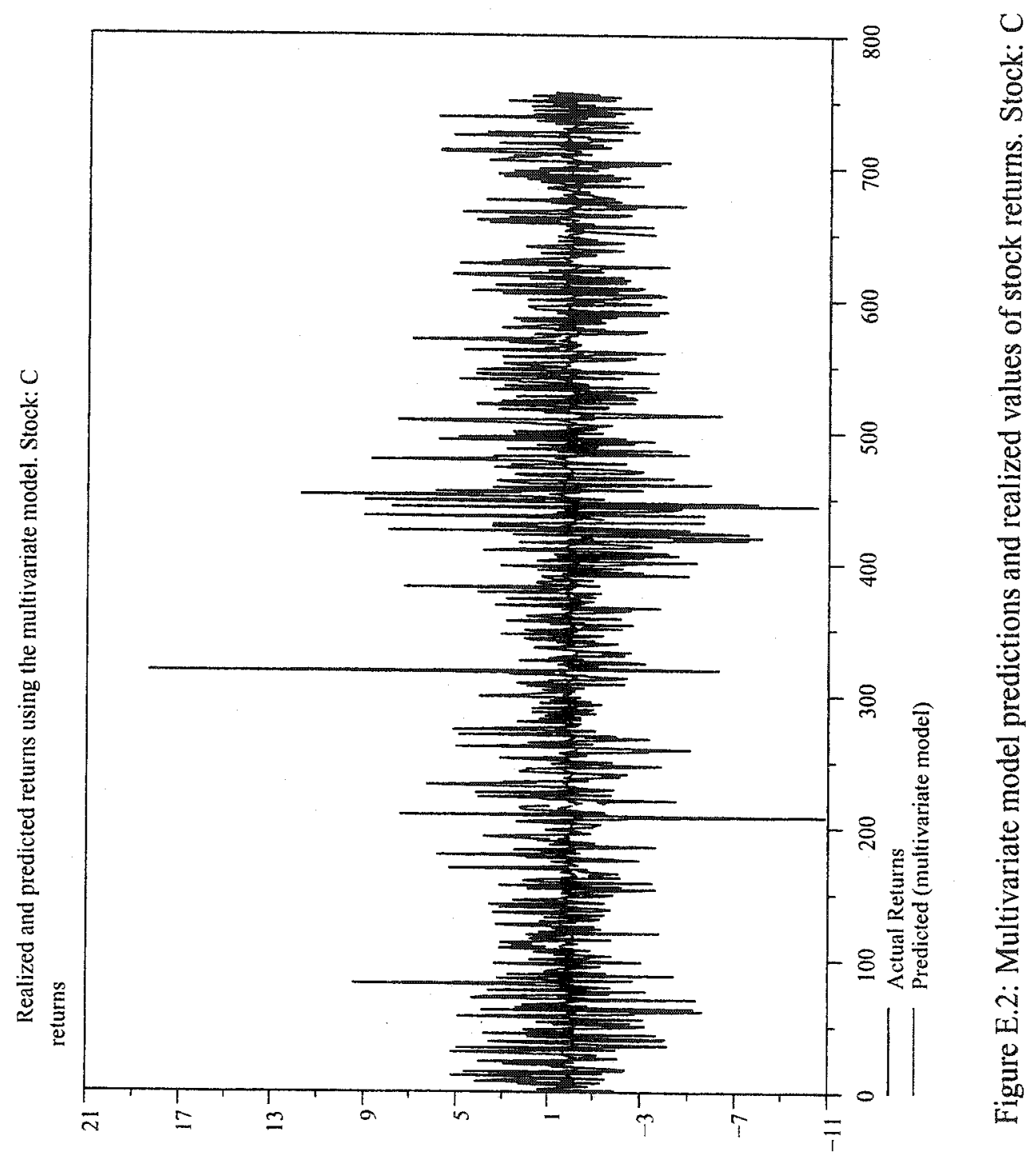




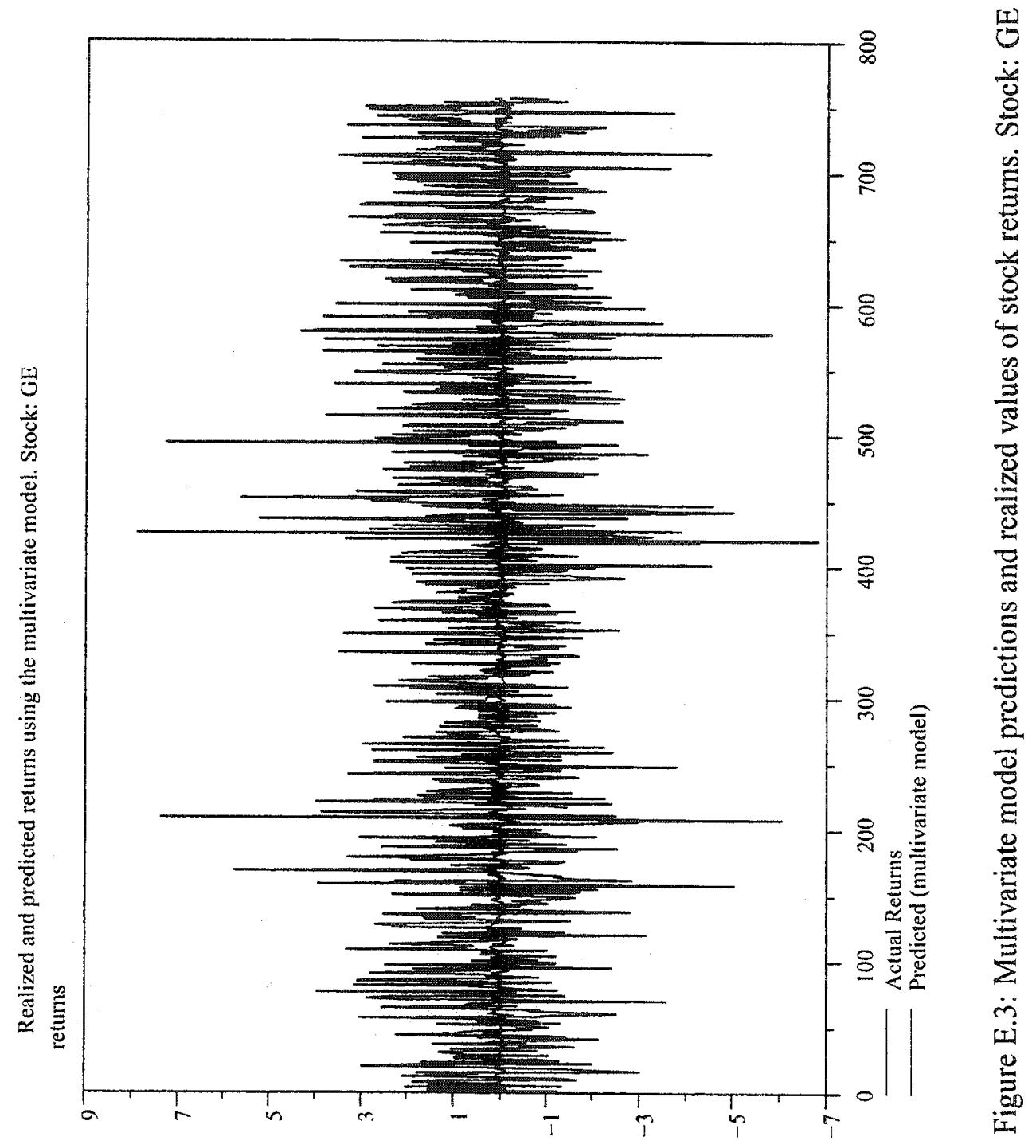




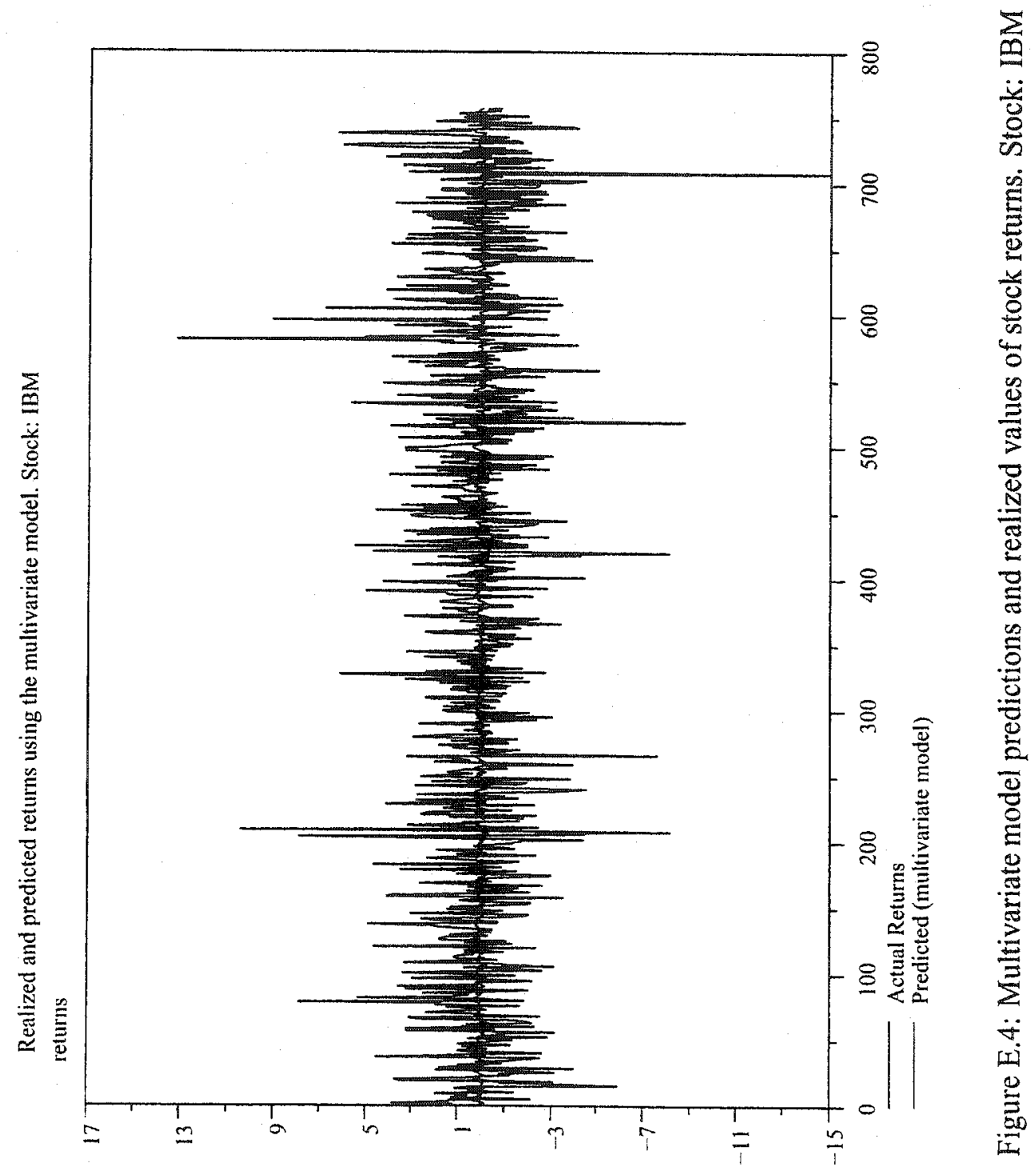




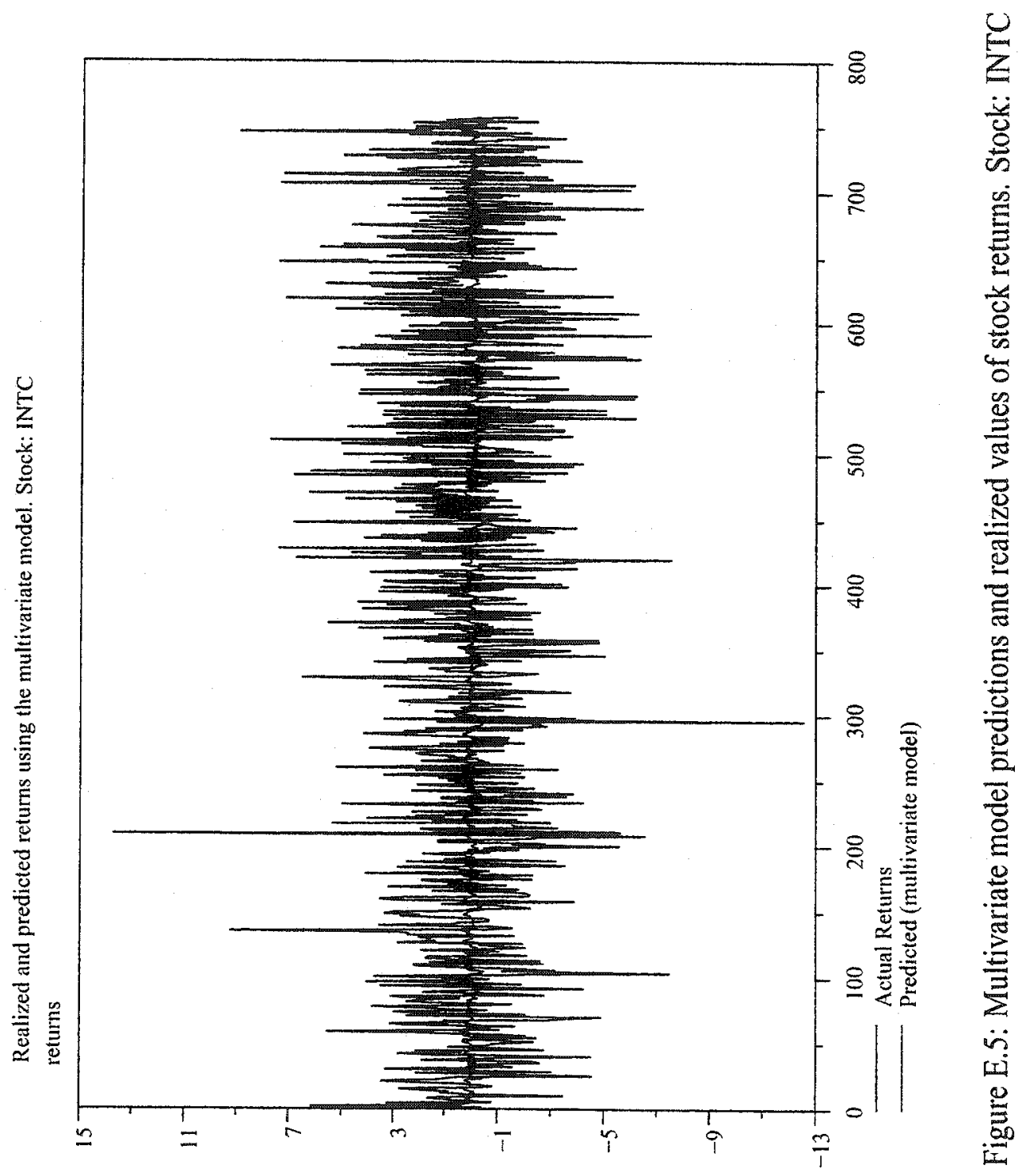




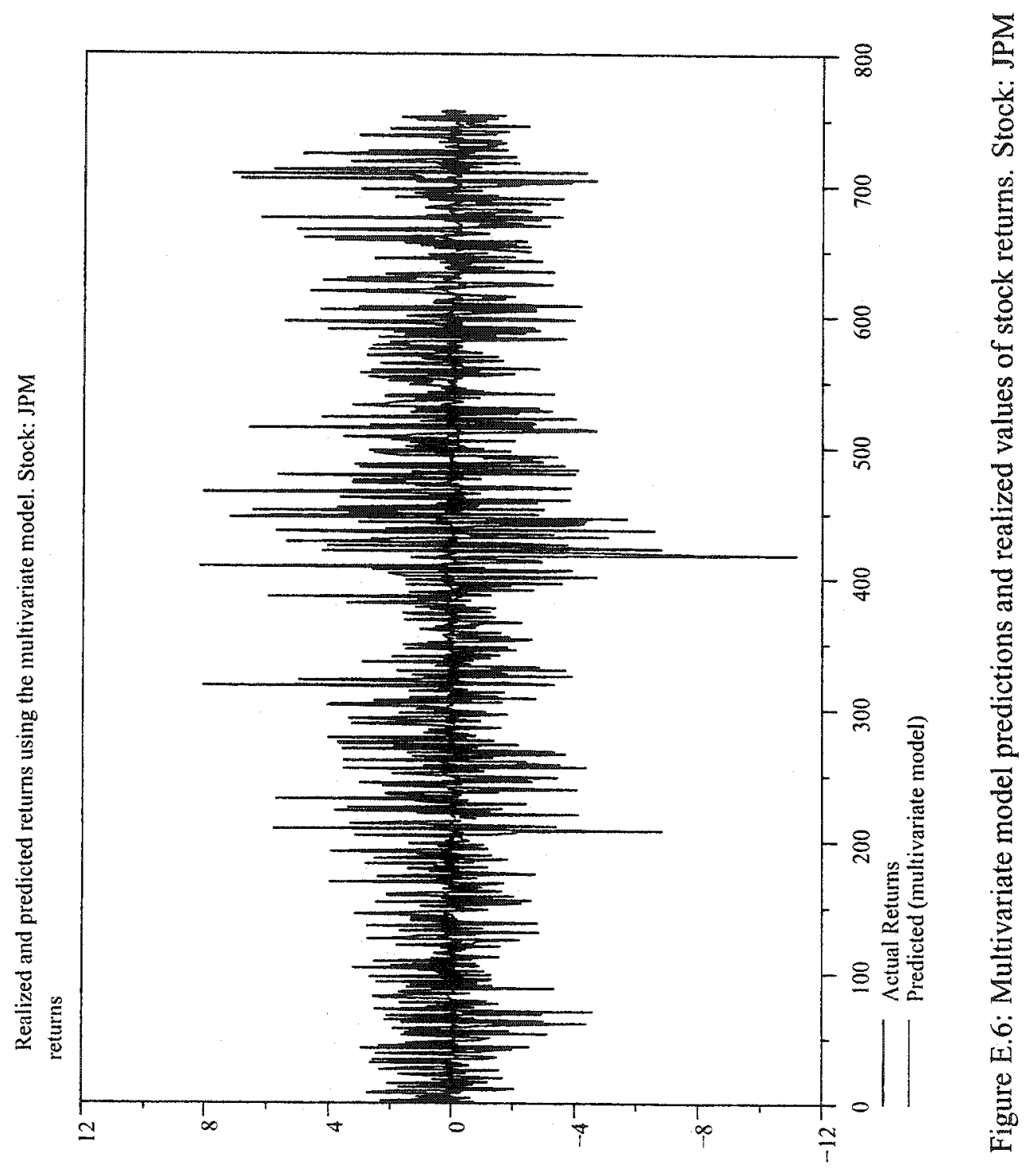




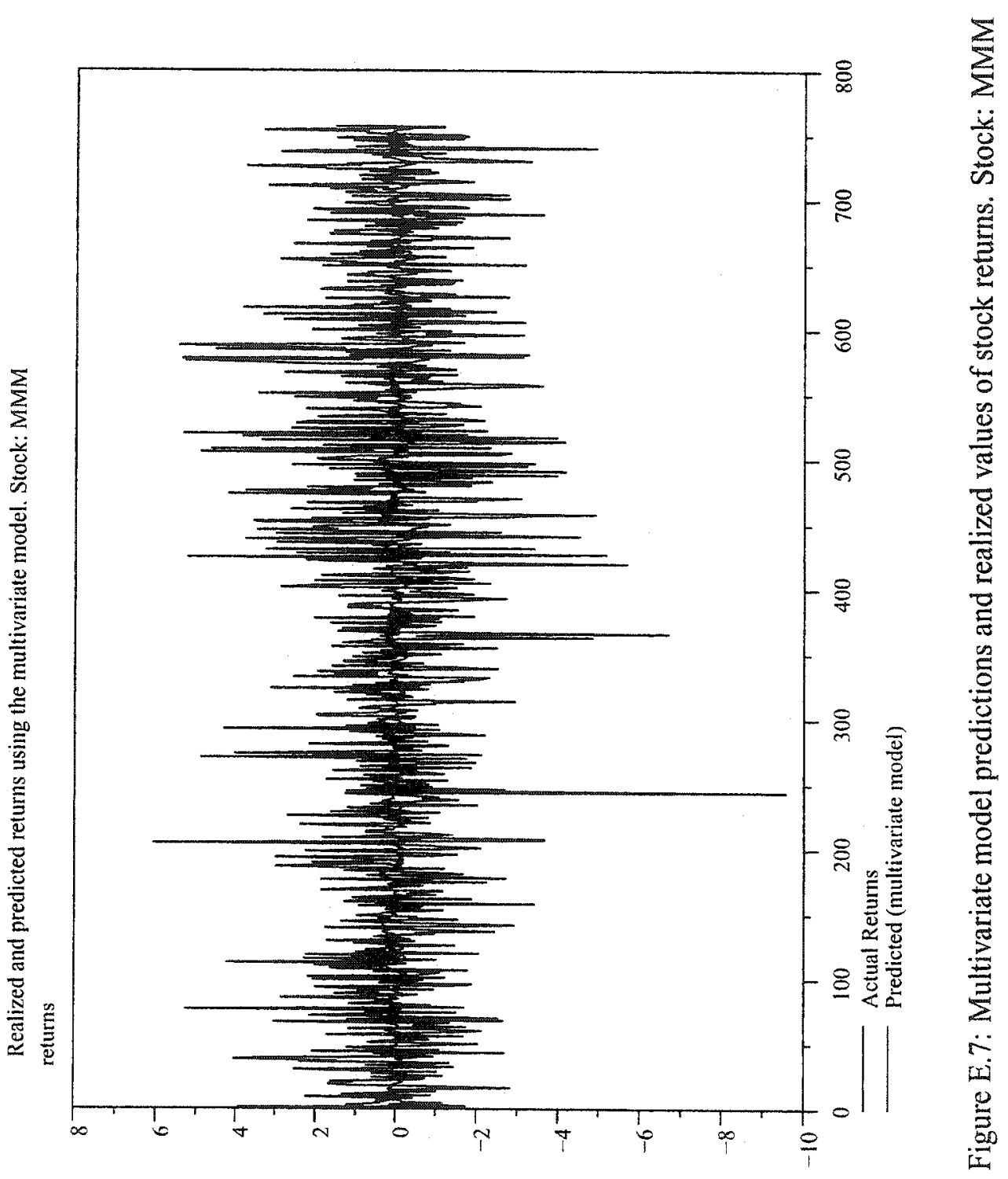




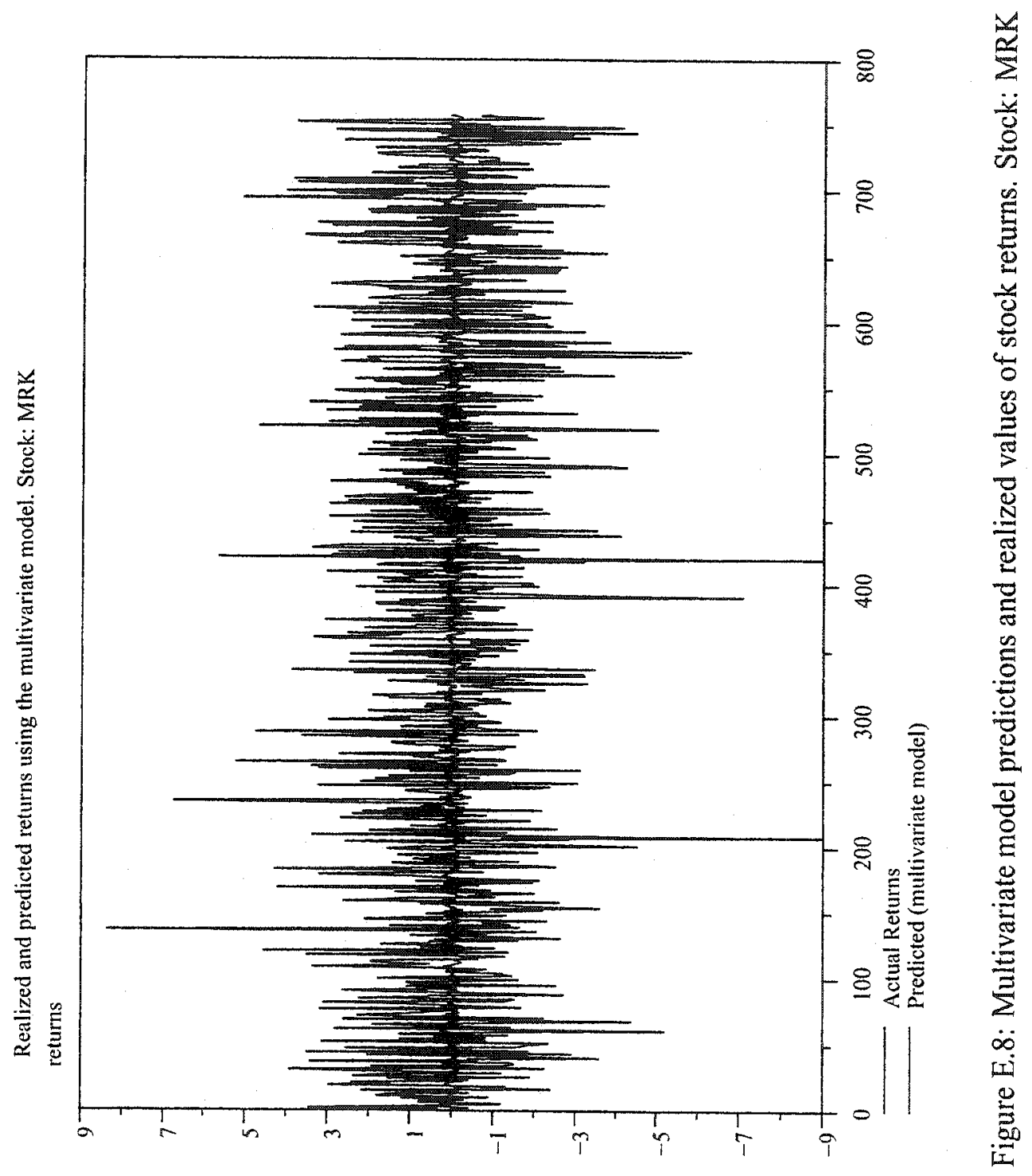




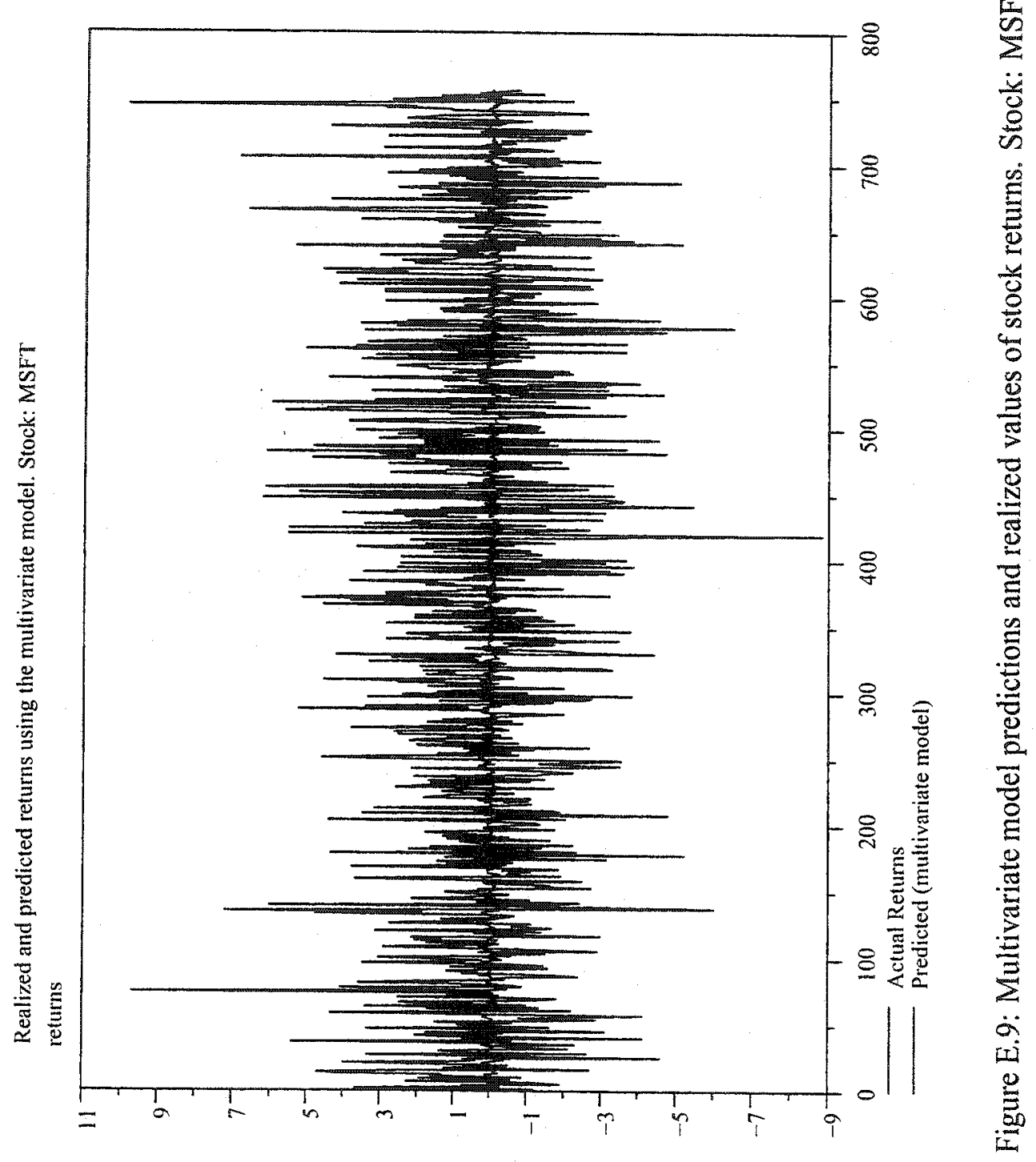




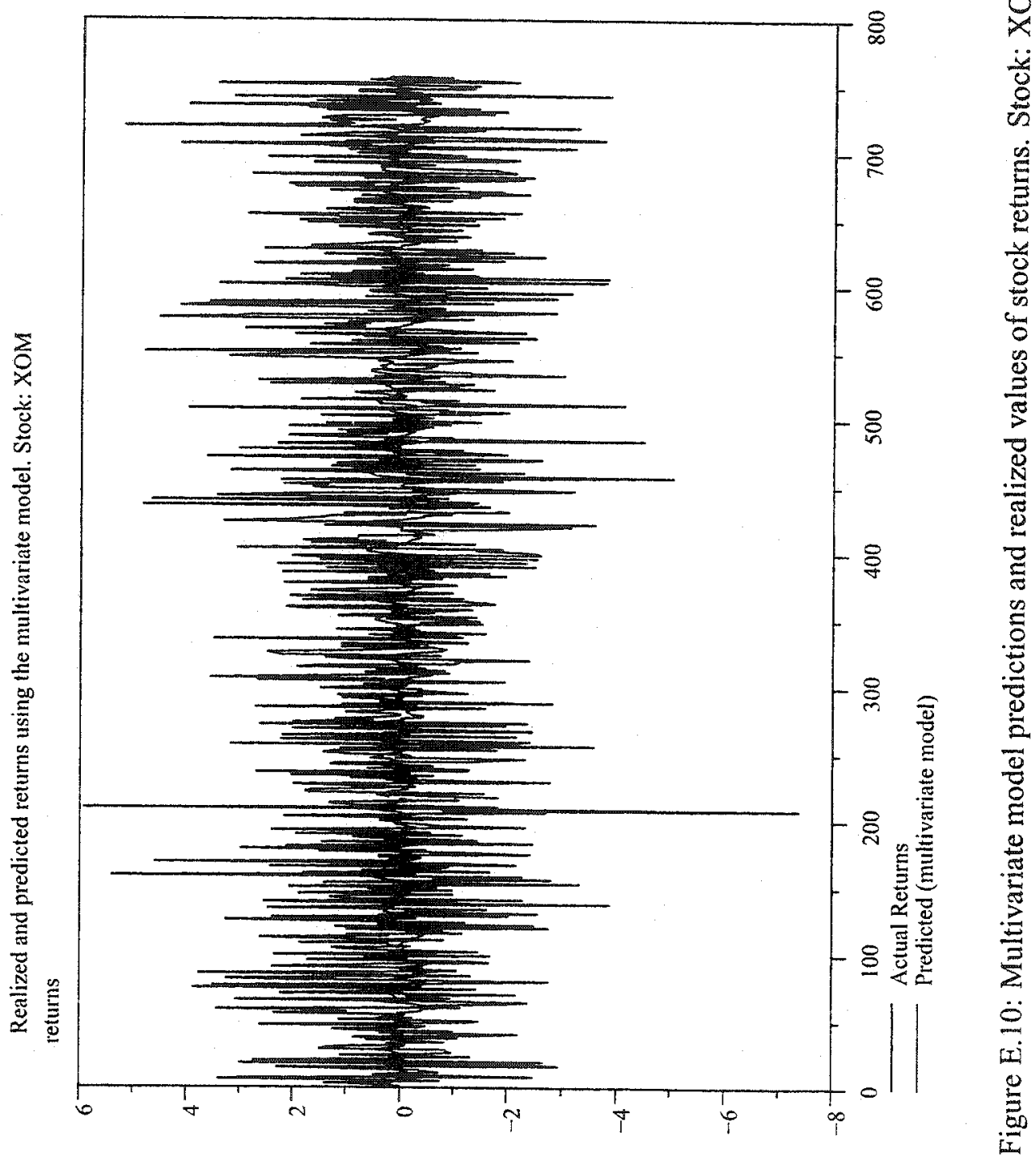


GARCH model return forecasts. Stock:BA

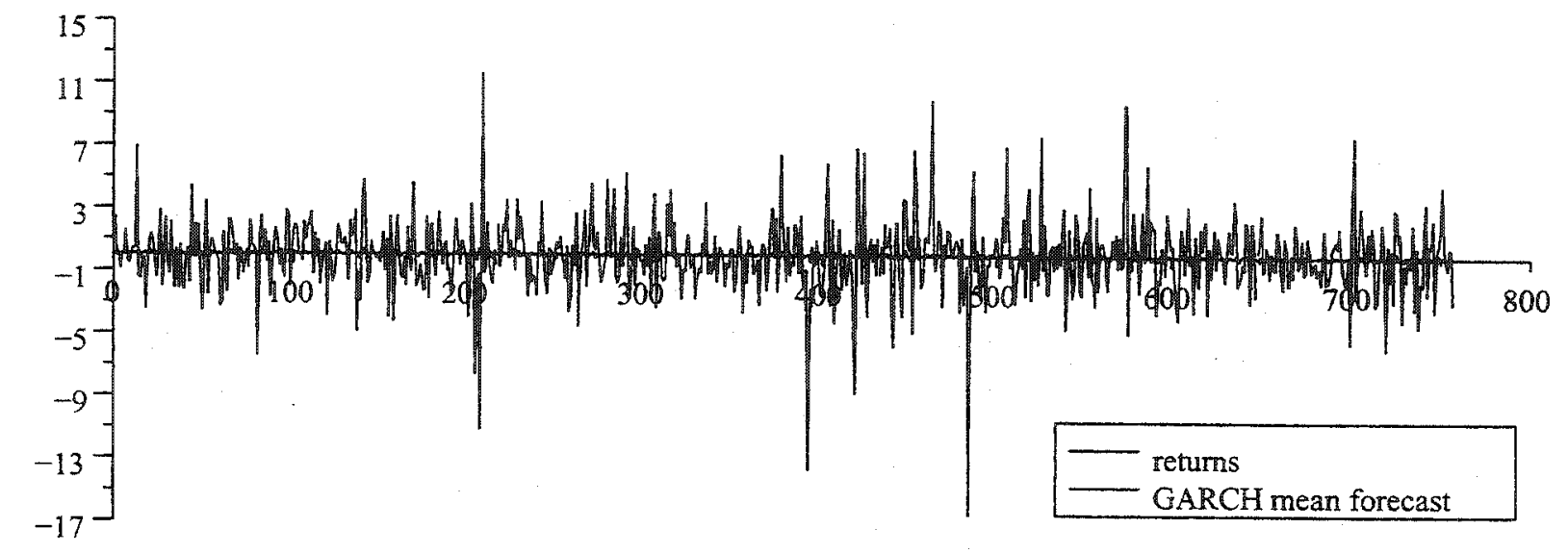

GJR-ARCH model return forecasts. Stock:BA

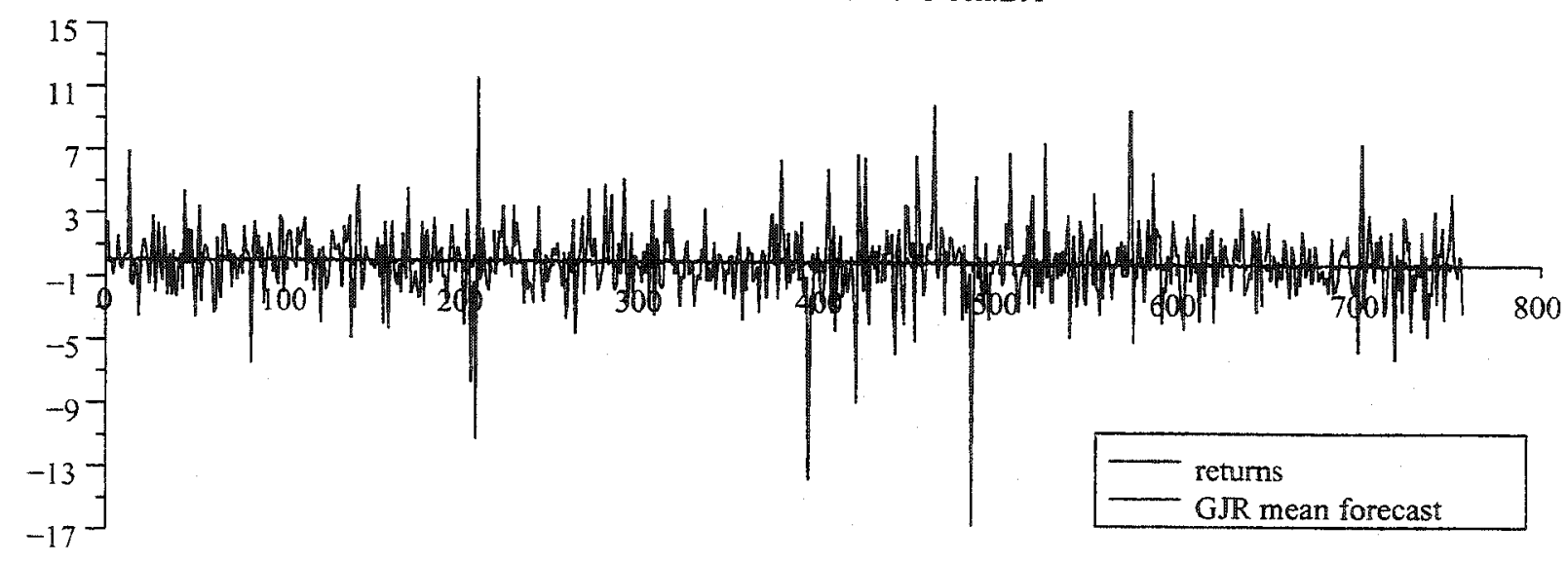

Figure E.11: GARCH and GIR-ARCH benchmark models stock returns forecasts. Stock: BA 

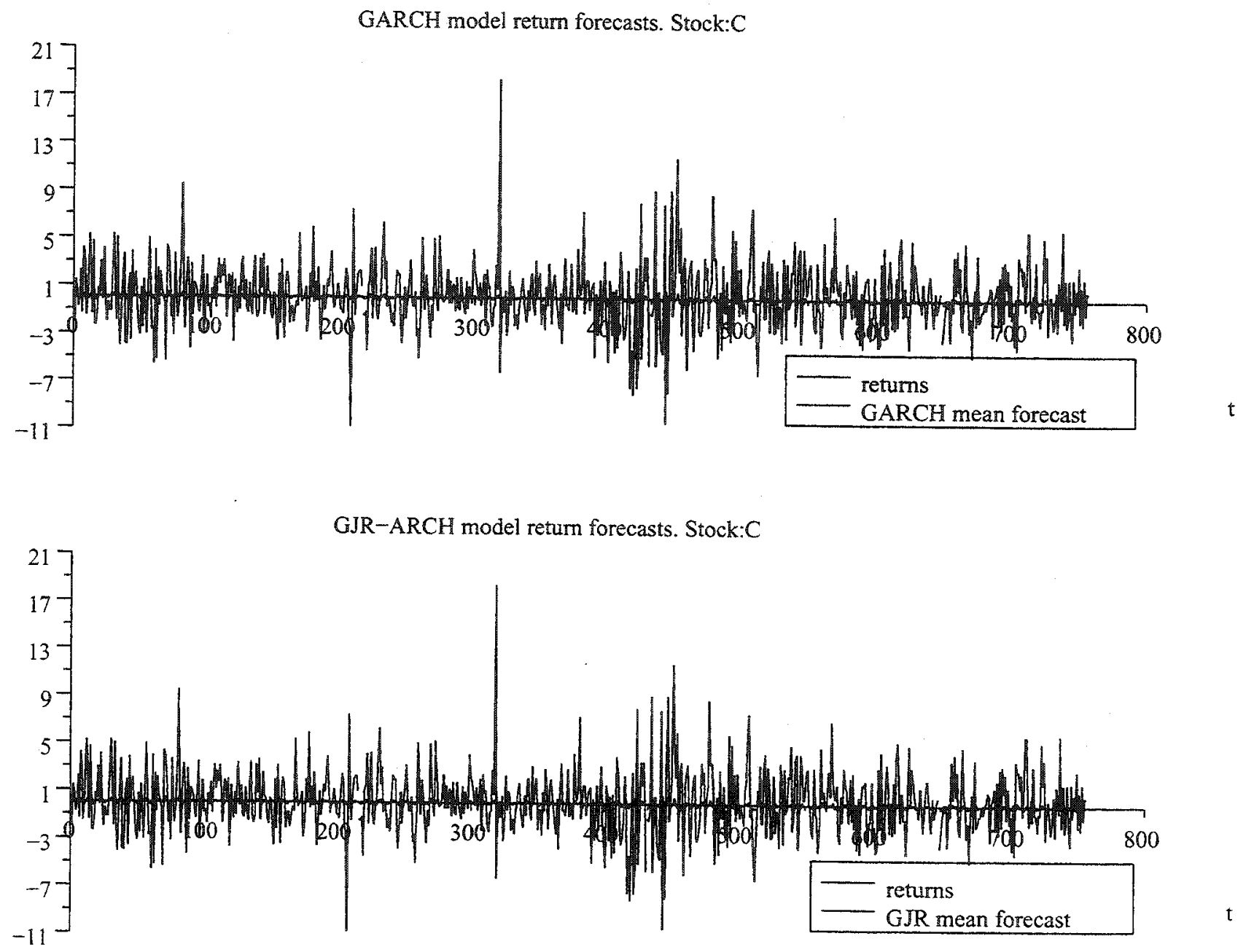

Figure E.12: GARCH and GJR-ARCH benchmark models stock returns forecasts. Stock: C 

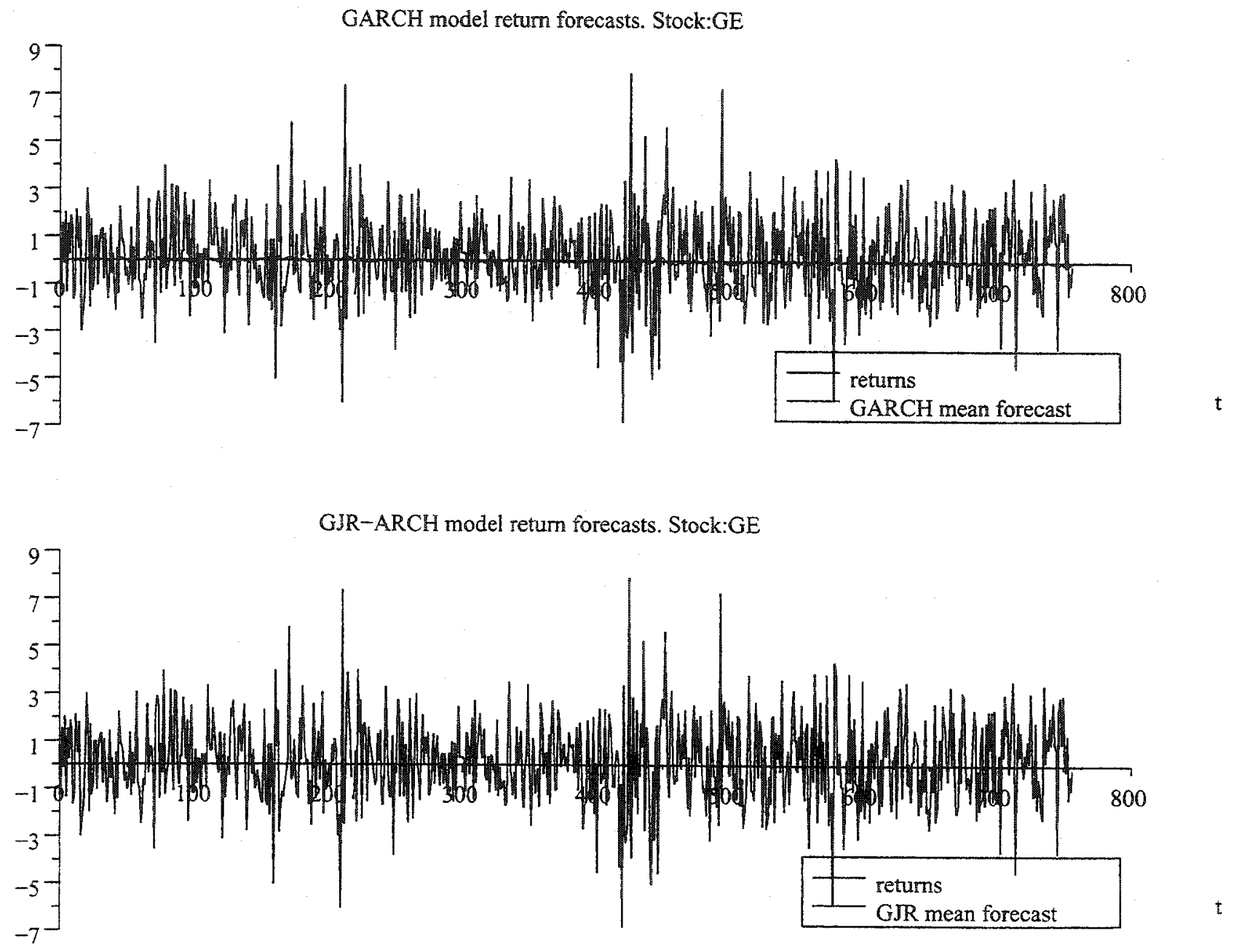

Figure E.13: GARCH and GJR-ARCH benchmark models stock returns forecasts. Stock: GE 

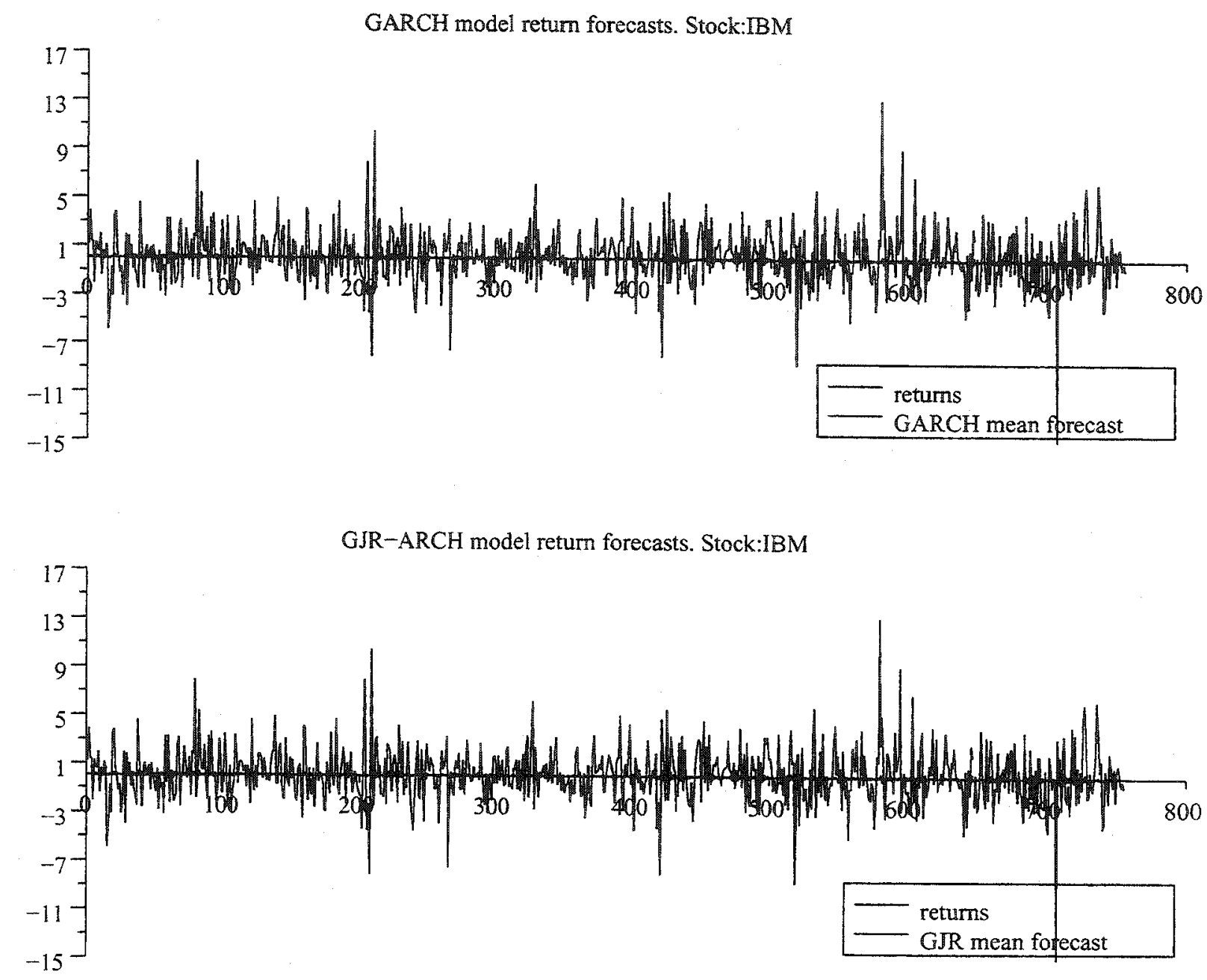

Figure E.14: GARCH and GJR-ARCH benchmark models stock returns forecasts. Stock: IBM 


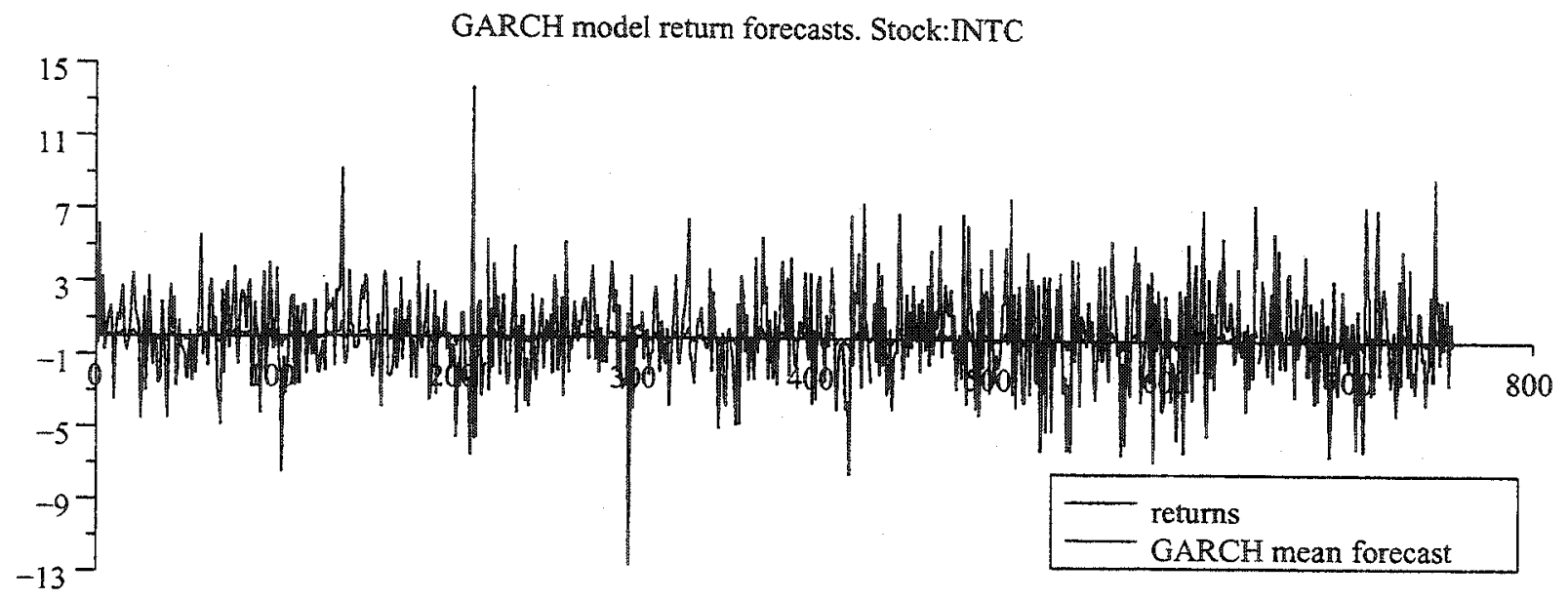

GJR-ARCH model return forecasts. Stock:INTC

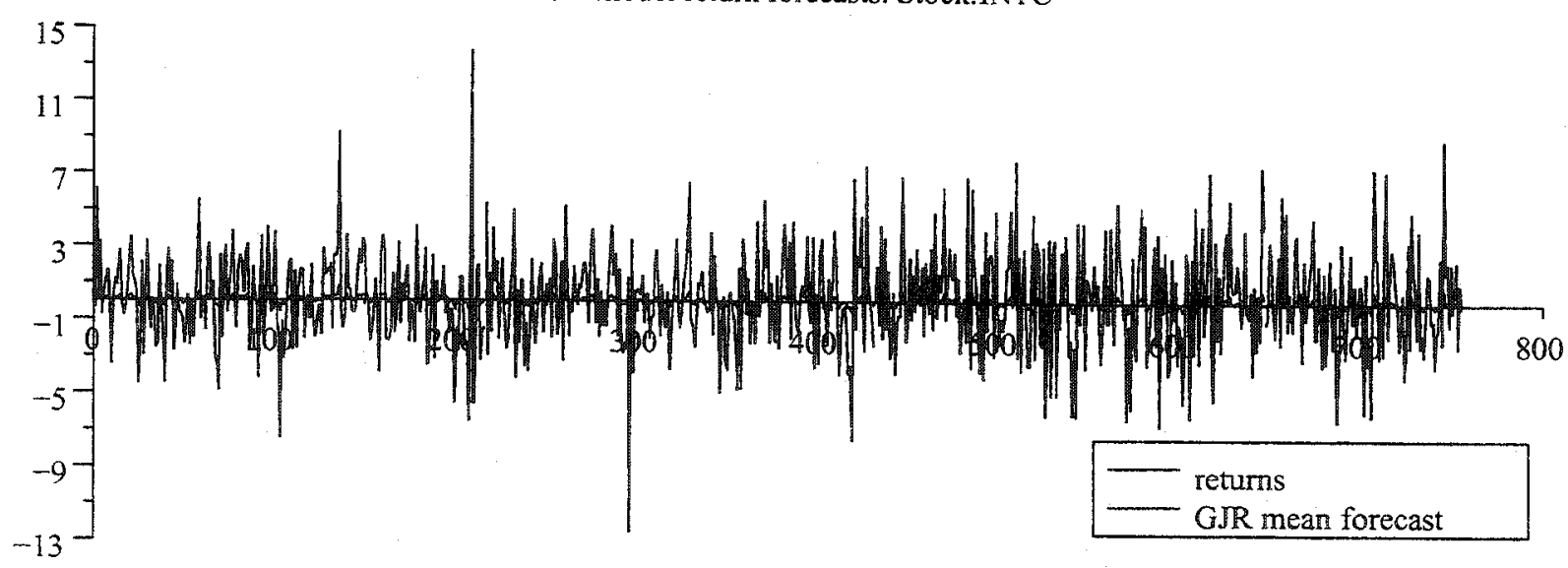

Figure E.15: GARCH and GJR-ARCH benchmark models stock returns forecasts. Stock: INTC 

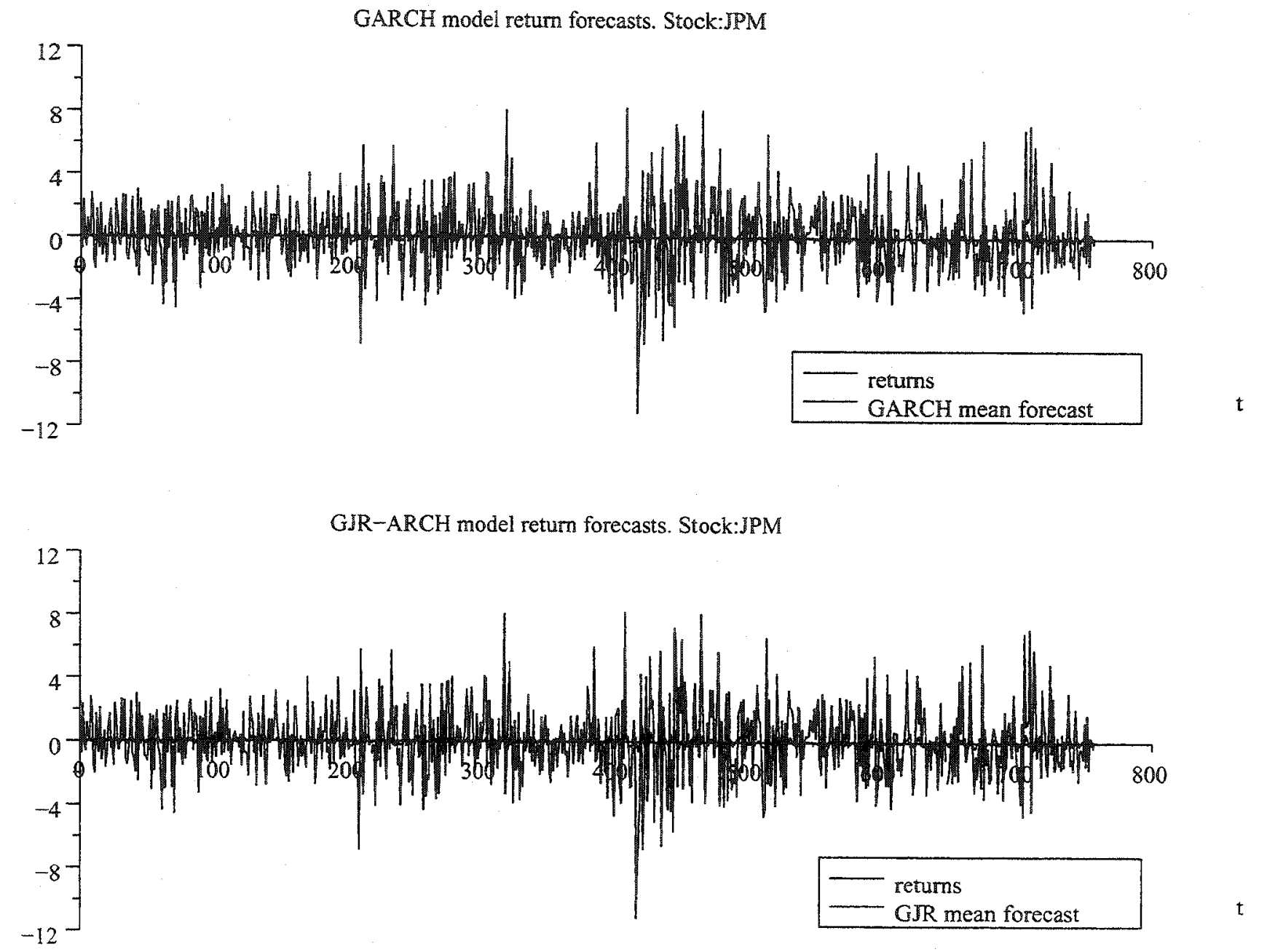

Figure E.16: GARCH and GJR-ARCH benchmark models stock returns forecasts. Stock: JPM 

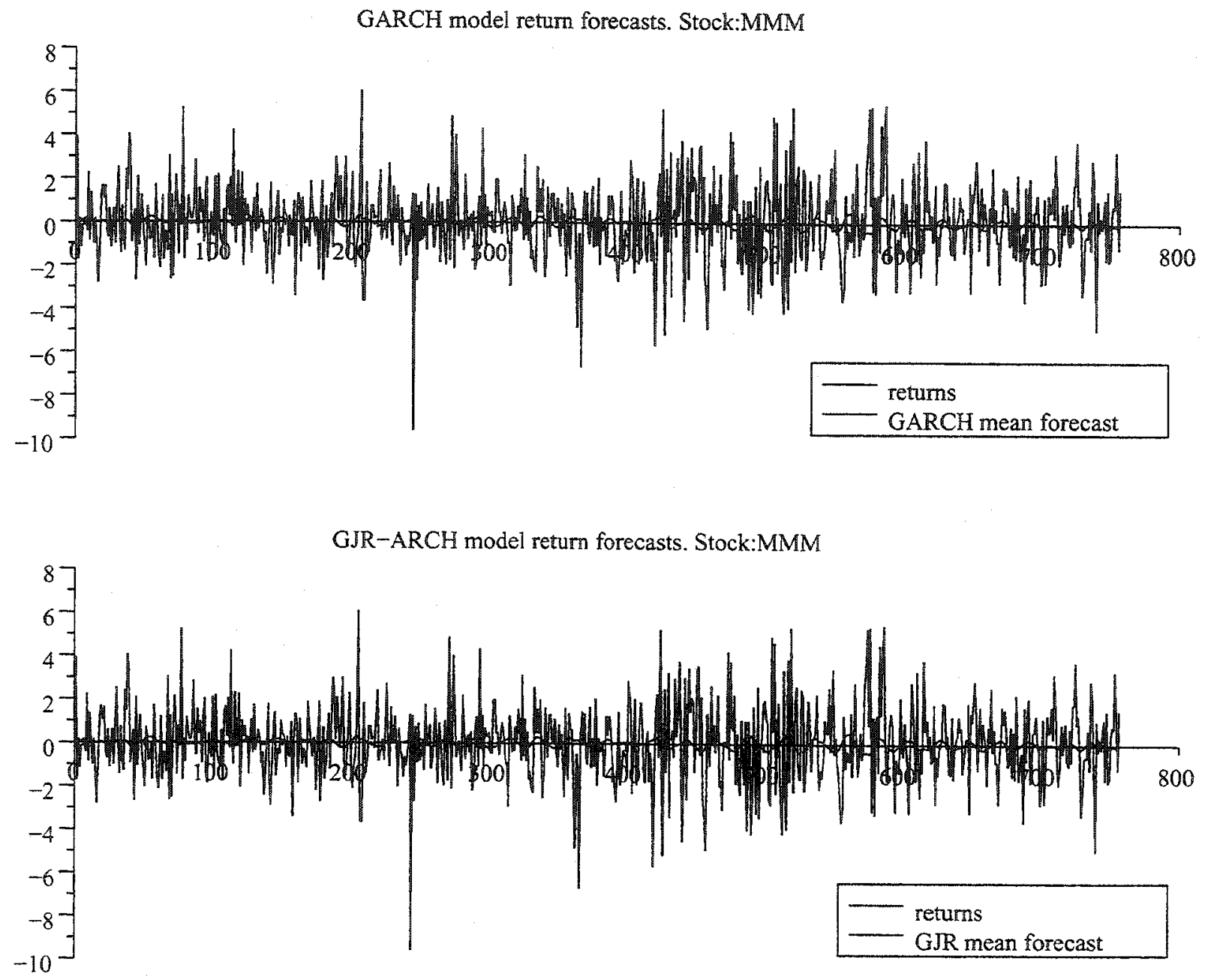

Figure E. 17: GARCH and GJR-ARCH benchmark models stock returns forecasts. Stock: MMM 

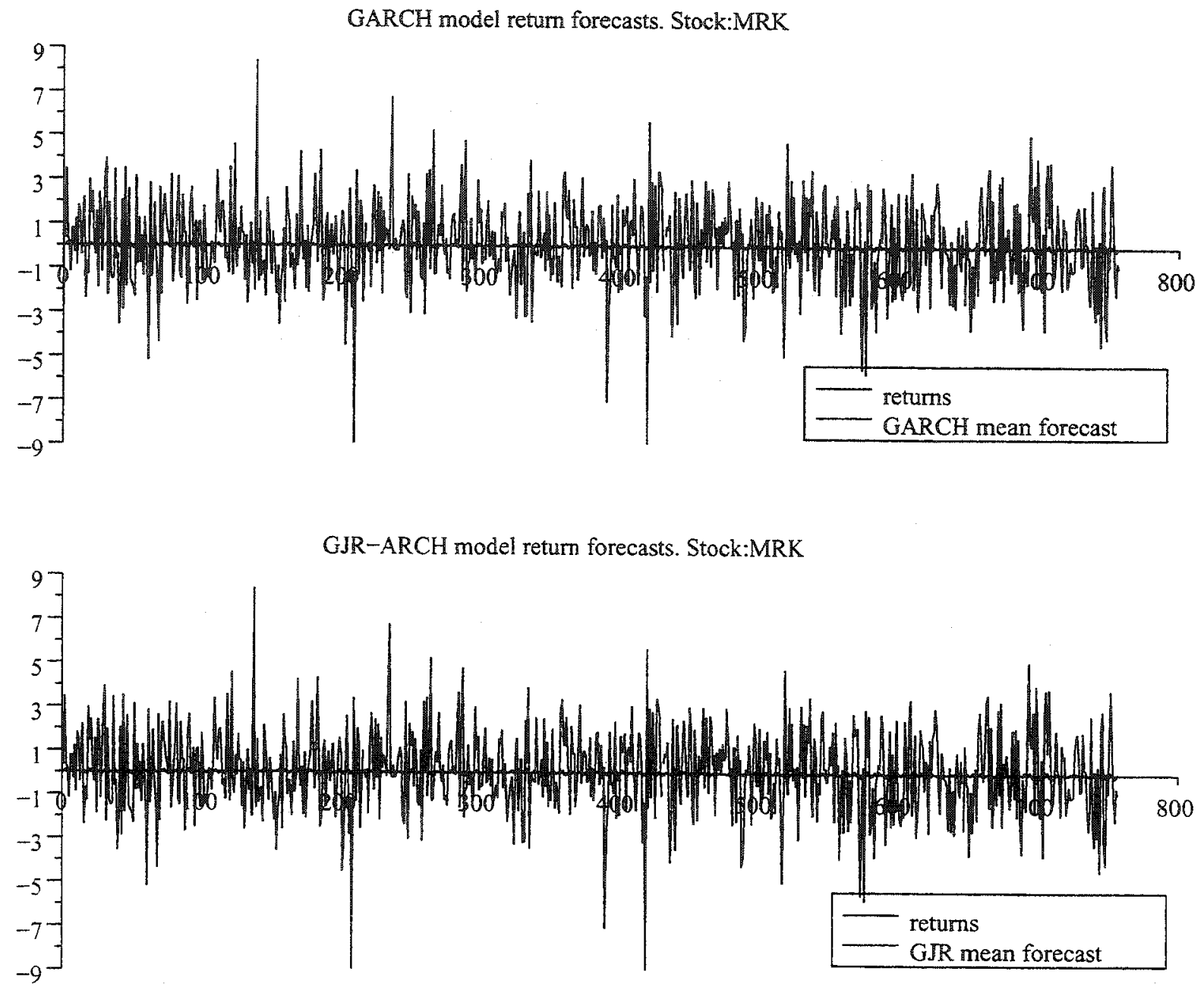

Figure E.18: GARCH and GJR-ARCH benchmark models stock returns forecasts. Stock: MRK 

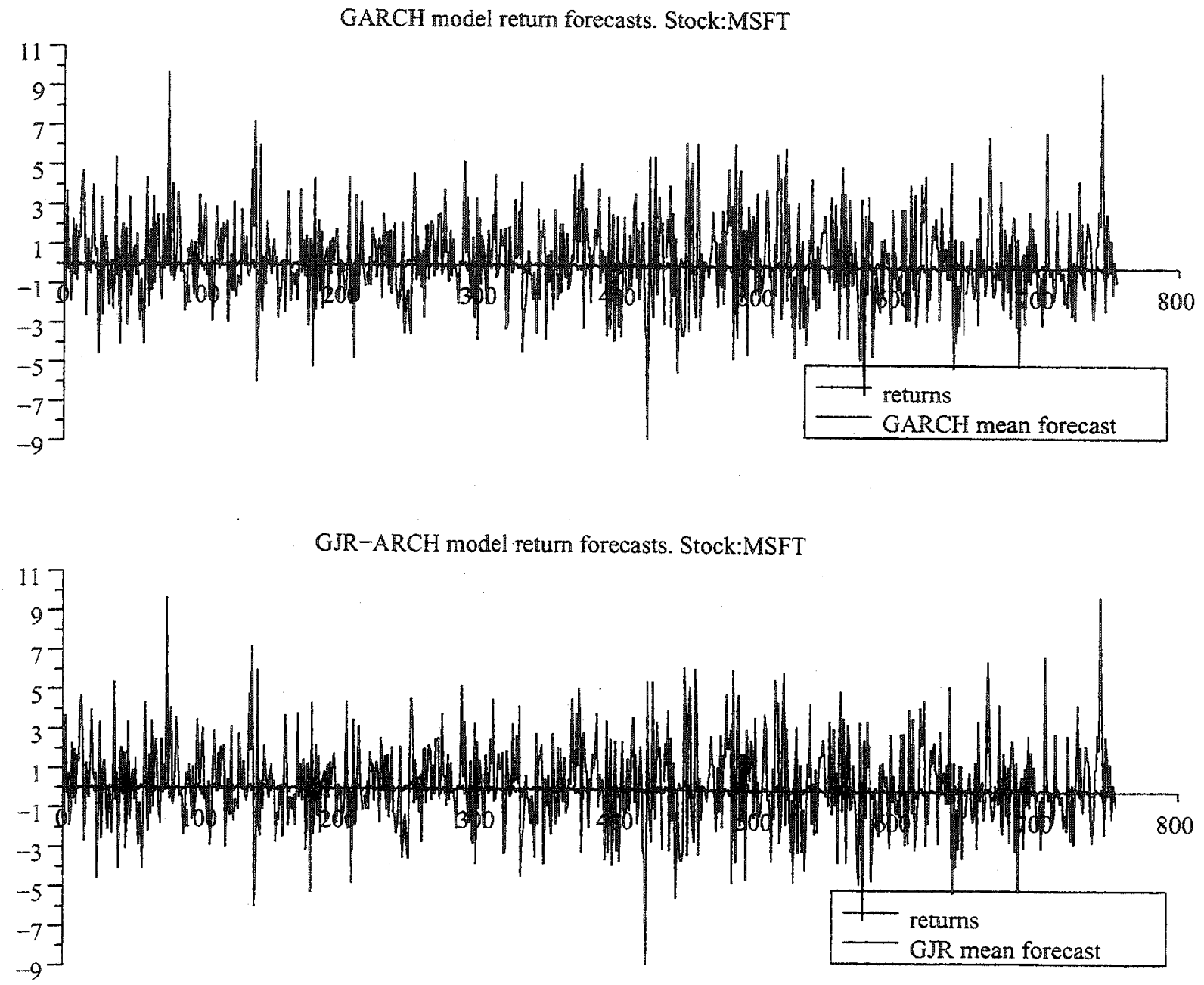

Figure E.19: GARCH and GJR-ARCH benchmark models stock returns forecasts. Stock: MSFT 

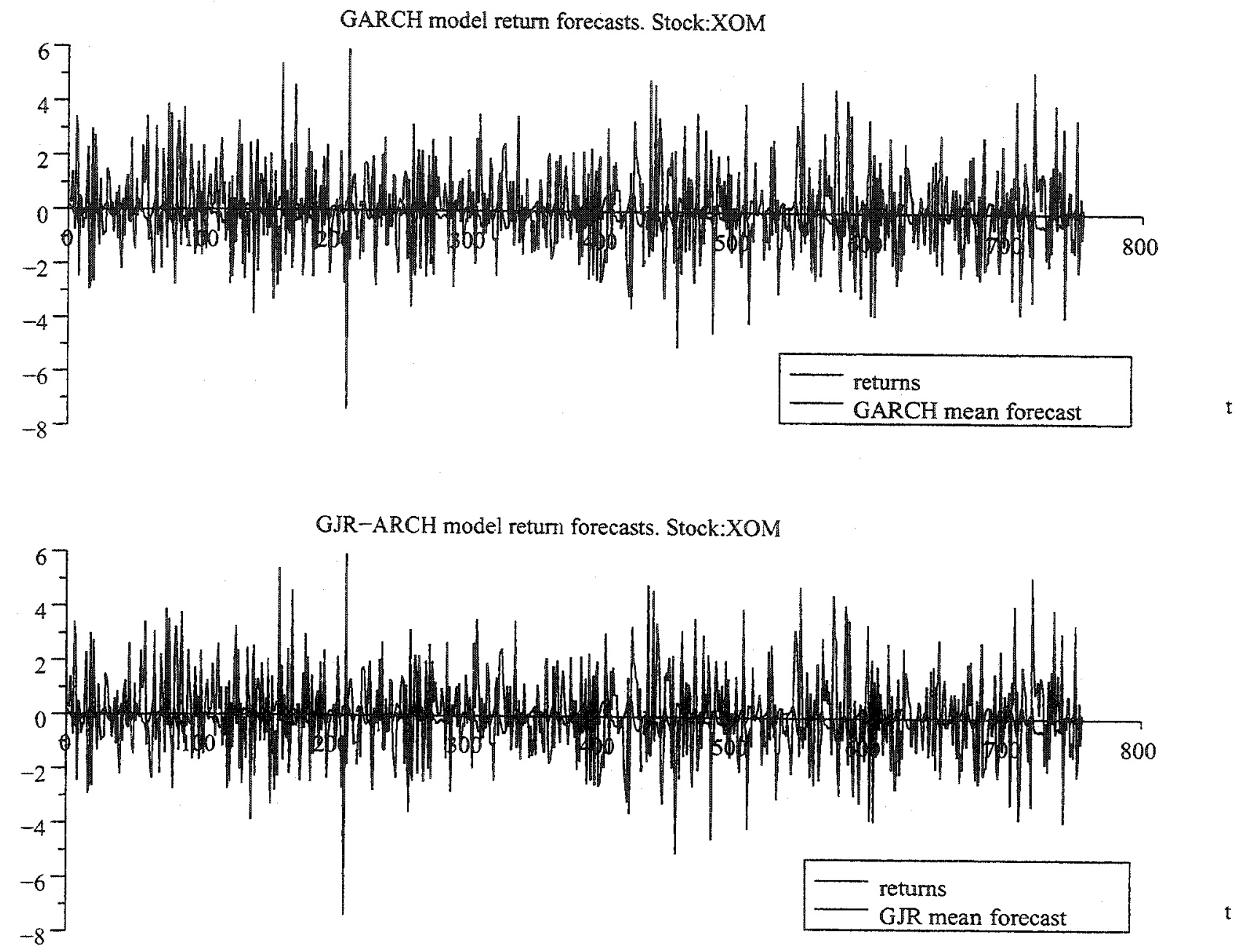

Figure E.20: GARCH and GJR-ARCH benchmark models stock returns forecasts. Stock: XOM 


\section{Appendix F}

\section{Sensitivity Analysis}

\begin{tabular}{|ll|l|l|l|l|l|lll|}
\hline \hline $\mathrm{p}$ & $\mathrm{q}$ & $\mathrm{AR} 1$ & $\mathrm{MA} 1$ & $\mathrm{MA} 2$ & $\mathrm{Q}$ & $\mathrm{R}$ & $\mathrm{LL}$ & $\mathrm{AIC}$ & $\mathrm{BIC}$ \\
\hline 1 & 2 & -0.276 & 0.309 & -0.031 & 2.474 & 0.01 & -3319 & 6646 & 6668 \\
1 & 2 & -0.218 & 0.250 & -0.039 & 2.433 & 0.05 & -3318.8 & 6645.7 & 6667.6 \\
1 & 2 & 0.215 & -0.182 & -0.064 & 2.279 & 0.2 & -3317.8 & 6643.7 & 6665.6 \\
1 & 2 & 0.351 & -0.317 & -0.069 & 2.178 & 0.3 & -3317.8 & 6643.6 & 6665.5 \\
1 & 2 & 0.292 & -0.254 & -0.075 & 1.977 & 0.5 & -3317.9 & 6643.8 & 6665.7 \\
1 & 2 & 0.298 & -0.248 & -0.094 & 1.474 & 1 & -3318.2 & 6644.4 & 6666.3 \\
\hline
\end{tabular}

Table F.1: Univariate model sensitivity analysis: parameter estimates for selected values of $R$. Stock:BA

Table features univariate model parameter estimates for selected values of the measurement noise variance $R$. The model's likelihood as well as the AIC and BIC fit criteria are presented. 


\begin{tabular}{|ll|l|l|l|l|l|lll|}
\hline \hline $\mathrm{p}$ & $\mathrm{q}$ & $\mathrm{AR} 1$ & $\mathrm{AR} 2$ & $\mathrm{MA} \mathrm{I}$ & $\mathrm{Q}$ & $\mathrm{R}$ & $\mathrm{LL}$ & $\mathrm{AIC}$ & $\mathrm{BIC}$ \\
\hline 2 & 1 & -0.300 & 0.021 & 0.368 & 3.924 & 0.01 & -3726.4 & 7460.8 & 7482.7 \\
2 & 1 & 0.193 & -0.026 & -0.123 & 3.883 & 0.05 & -3726.1 & 7460.2 & 7482.1 \\
2 & 1 & 0.694 & -0.068 & -1.597 & 1.460 & 0.2 & -3728.1 & 7464.2 & 7486.1 \\
2 & 1 & 0.639 & -0.065 & -1.755 & 1.176 & 0.3 & -3728.3 & 7464.6 & 7486.5 \\
2 & 1 & 0.618 & -0.067 & -1.839 & 1.013 & 0.5 & -3728.4 & 7464.9 & 7486.8 \\
2 & 1 & 0.600 & -0.075 & -1.949 & 0.768 & 1 & -3728.7 & 7465.3 & 7487.3 \\
\hline
\end{tabular}

Table F.2: Univariate model sensitivity analysis: parameter estimates for selected values of $R$. Stock:C

Table features univariate model parameter estimates for selected values of the measurement noise variance $R$. The model's likelihood as well as the AIC and BIC fit criteria are presented.

\begin{tabular}{|ll|l|l|l|l|l|lll|}
\hline \hline $\mathrm{p}$ & $\mathrm{q}$ & $\mathrm{AR} 1$ & MA 1 & MA 2 & $\mathrm{Q}$ & $\mathrm{R}$ & $\mathrm{LL}$ & $\mathrm{AIC}$ & $\mathrm{BIC}$ \\
\hline 2 & 1 & -0.384 & 0.012 & 0.425 & 1.524 & 0.01 & -2893.3 & 5794.6 & 5816.6 \\
2 & 1 & 0.045 & -0.036 & -0.020 & 1.483 & 0.05 & -2892.6 & 5793.2 & 5815.1 \\
2 & 1 & 0.506 & -0.053 & -0.473 & 1.330 & 0.2 & -2891.5 & 5791 & 5812.9 \\
2 & 1 & 0.605 & -0.057 & -0.570 & 1.229 & 0.3 & -2891.3 & 5790.5 & 5812.5 \\
2 & 1 & 0.655 & -0.064 & -0.616 & 1.028 & 0.5 & -2891.1 & 5790.3 & 5812.2 \\
2 & 1 & 0.792 & -0.089 & -0.730 & 0.526 & 1 & -2891.2 & 5790.3 & 5812.2 \\
\hline
\end{tabular}

Table F.3: Univariate model sensitivity analysis: parameter estimates for selected values of $R$. Stock:GE

Table features univariate model parameter estimates for selected values of the measurement noise variance $R$. The model's likelihood as well as the AIC and $\mathrm{BIC}$ fit criteria are presented. 


\begin{tabular}{|ll|l|l|l|lll|}
\hline \hline $\mathrm{p}$ & $\mathrm{q}$ & $\mathrm{AR} \mathrm{1}$ & $\mathrm{Q}$ & $\mathrm{R}$ & $\mathrm{LL}$ & $\mathrm{AIC}$ & $\mathrm{BIC}$ \\
\hline 1 & 0 & 0.033 & 2.975 & 0.01 & -3482 & 6967.9 & 6978.9 \\
1 & 0 & 0.034 & 2.935 & 0.05 & -3481.9 & 6967.9 & 6978.9 \\
1 & 0 & 0.036 & 2.784 & 0.2 & -3481.9 & 6967.8 & 6978.8 \\
1 & 0 & 0.038 & 2.683 & 0.3 & -3481.9 & 6967.7 & 6978.7 \\
1 & 0 & 0.041 & 2.482 & 0.5 & -3481.8 & 6967.6 & 6978.6 \\
1 & 0 & 0.049 & 1.979 & 1 & -3481.6 & 6967.2 & 6978.2 \\
\hline
\end{tabular}

Table F.4: Univariate model sensitivity analysis: parameter estimates for selected values of $R$. Stock:IBM

Table features univariate model parameter estimates for selected values of the measurement noise variance $R$. The model's likelihood as well as the AIC and $\mathrm{BIC}$ fit criteria are presented.

\begin{tabular}{|ll|l|l|l|l|l|lll|}
\hline \hline $\mathrm{p}$ & $\mathrm{q}$ & $\mathrm{AR} 1$ & $\mathrm{MA} 1$ & $\mathrm{MA} 2$ & $\mathrm{Q}$ & $\mathrm{R}$ & $\mathrm{LL}$ & $\mathrm{AIC}$ & $\mathrm{BIC}$ \\
\hline 1 & 2 & -0.363 & 0.452 & 0.016 & 5.439 & 0.01 & -4014.7 & 8037.4 & 8059.3 \\
1 & 2 & 0.004 & 0.081 & -0.033 & 5.392 & 0.05 & -4014.1 & 8036.1 & 8058 \\
1 & 2 & 0.299 & -2.529 & -0.416 & 0.725 & 0.2 & -4017 & 8041.9 & 8063.8 \\
1 & 2 & 0.116 & -4.577 & -0.752 & 0.423 & 0.3 & -4144.6 & 8297.2 & 8319.1 \\
1 & 2 & N/A & N/A & N/A & N/A & N/A & N/A & N/A & N/A \\
1 & 2 & -1.168 & 21.16 & 24.293 & 18.372 & 1 & -9856.9 & 19722 & 19744 \\
\hline \hline
\end{tabular}

Table F.5: Univariate model sensitivity analysis: parameter estimates for selected values of R. Stock:INTC

Table features univariate model parameter estimates for selected values of the measurement noise variance $R$. The model's likelihood as well as the AIC and $\mathrm{BIC}$ fit criteria are presented. 


\begin{tabular}{|ll|l|l|l|l|l|l|lll|}
\hline \hline $\mathrm{p}$ & $\mathrm{q}$ & $\mathrm{AR} 1$ & $\mathrm{MA} \mathrm{1}$ & $\mathrm{MA} 2$ & $\mathrm{MA} \mathrm{3}$ & $\mathrm{Q}$ & $\mathrm{R}$ & $\mathrm{LL}$ & $\mathrm{AIC}$ & $\mathrm{BIC}$ \\
\hline 1 & 3 & -0.604 & 0.685 & 0.024 & -0.014 & 2.075 & 0.01 & -3164.2 & 6338.5 & 6365.9 \\
1 & 3 & 0.455 & -0.376 & -0.065 & -0.040 & 2.026 & 0.05 & -3160.8 & 6331.5 & 6358.9 \\
1 & 3 & 0.543 & -0.458 & -0.078 & -0.039 & 1.873 & 0.2 & -3160.5 & 6331 & 6358.4 \\
1 & 3 & 0.574 & -0.484 & -0.085 & -0.040 & 1.772 & 0.3 & -3160.5 & 6331 & 6358.3 \\
1 & 3 & 0.616 & -0.516 & -0.100 & -0.042 & 1.568 & 0.5 & -3160.5 & 6331 & 6358.4 \\
1 & 3 & 0.699 & -0.555 & -0.155 & -0.052 & 1.053 & 1 & -3160.7 & 6331.3 & 6358.7 \\
\hline \hline
\end{tabular}

Table F.6: Univariate model sensitivity analysis: parameter estimates for selected values of R. Stock:JPM

Table features univariate model parameter estimates for selected values of the measurement noise variance $R$. The model's likelihood as well as the AIC and BIC fit criteria are presented.

\begin{tabular}{|ll|l|l|l|l|lll|}
\hline \hline $\mathrm{p}$ & $\mathrm{q}$ & $\mathrm{AR} 1$ & $\mathrm{MA} 1$ & $\mathrm{Q}$ & $\mathrm{R}$ & $\mathrm{LL}$ & $\mathrm{AIC}$ & $\mathrm{BIC}$ \\
\hline 1 & 1 & -0.291 & 0.275 & 1.389 & 0.01 & -2810.4 & 5626.8 & 5643.3 \\
1 & 1 & 0.371 & -0.406 & 1.347 & 0.05 & -2808.9 & 5623.9 & 5640.3 \\
1 & 1 & 0.683 & -0.732 & 1.193 & 0.2 & -2807.4 & 5620.8 & 5637.3 \\
1 & 1 & 0.708 & -0.761 & 1.092 & 0.3 & -2807.4 & 5620.8 & 5637.2 \\
1 & 1 & 0.711 & -0.776 & 0.890 & 0.5 & -2807.5 & 5621.1 & 5637.5 \\
1 & 1 & 0.714 & -0.850 & 0.384 & 1 & -2808.5 & 5622.9 & 5639.3 \\
\hline
\end{tabular}

Table F.7: Univariate model sensitivity analysis: parameter estimates for selected values of $R$. Stock:MMM

Table features univariate model parameter estimates for selected values of the measurement noise variance $R$. The model's likelihood as well as the AIC and $\mathrm{BIC}$ fit criteria are presented. 


\begin{tabular}{|ll|l|l|l|l|l|l|lll|}
\hline \hline $\mathrm{p}$ & $\mathrm{q}$ & $\mathrm{AR} 1$ & $\mathrm{MA} \mathrm{1}$ & MA 2 & MA 3 & $\mathrm{Q}$ & $\mathrm{R}$ & $\mathrm{LL}$ & $\mathrm{AIC}$ & $\mathrm{BIC}$ \\
\hline 1 & 3 & -0.904 & 0.904 & 0.002 & 0.002 & 2.378 & 0.01 & -3283.6 & 6577.2 & 6604.6 \\
1 & 3 & 0.074 & -0.078 & -0.004 & -0.053 & 2.328 & 0.05 & -3280.9 & 6571.9 & 6599.3 \\
1 & 3 & 0.223 & -0.227 & -0.004 & -0.058 & 2.177 & 0.2 & -3280.9 & 6571.8 & 6599.2 \\
1 & 3 & 0.224 & -0.229 & -0.004 & -0.061 & 2.077 & 0.3 & -3280.9 & 6571.8 & 6599.2 \\
1 & 3 & 0.231 & -0.237 & -0.005 & -0.066 & 1.875 & 0.5 & -3281 & 6572 & 6599.4 \\
1 & 3 & 0.258 & -0.267 & -0.009 & -0.080 & 1.372 & 1 & -3281.4 & 6572.7 & 6600.1 \\
\hline \hline
\end{tabular}

Table F.8: Univariate model sensitivity analysis: parameter estimates for selected values of R. Stock:MRK

Table features univariate model parameter estimates for selected values of the measurement noise variance $R$. The model's likelihood as well as the AIC and BIC fit criteria are presented.

\begin{tabular}{|ll|l|l|l|l|l|lll|}
\hline \hline $\mathrm{p}$ & $\mathrm{q}$ & $\mathrm{AR} 1$ & $\mathrm{MA} 1$ & $\mathrm{MA} 2$ & $\mathrm{Q}$ & $\mathrm{R}$ & $\mathrm{LI}$ & $\mathrm{AIC}$ & $\mathrm{BIC}$ \\
\hline 1 & 2 & -0.581 & 0.610 & -0.001 & 4.429 & 0.01 & -3832.9 & 7673.8 & 7695.8 \\
1 & 2 & -0.123 & 0.150 & -0.040 & 4.381 & 0.05 & -3831.1 & 7670.2 & 7692.1 \\
1 & 2 & 0.422 & -1.728 & -0.255 & 1.208 & 0.2 & -3832.5 & 7673 & 7694.9 \\
1 & 2 & 0.204 & -2.596 & -0.498 & 0.537 & 0.3 & -3833.7 & 7675.5 & 7697.4 \\
1 & 2 & -1.109 & -11.43 & -12.33 & 6.526 & 0.5 & -7764.3 & 15537 & 15559 \\
1 & 2 & N/A & N/A & N/A & N/A & N/A & N/A & N/A & N/A \\
\hline \hline
\end{tabular}

Table F.9: Univariate model sensitivity analysis: parameter estimates for selected values of R. Stock:MSFT

Table features univariate model parameter estimates for selected values of the measurement noise variance $R$. The model's likelihood as well as the AIC and BIC fit criteria are presented. 


\begin{tabular}{|ll|l|l|l|l|lll|}
\hline $\mathrm{p}$ & $\mathrm{q}$ & $\mathrm{AR} 1$ & $\mathrm{MA} \mathrm{1}$ & $\mathrm{Q}$ & $\mathrm{R}$ & $\mathrm{LL}$ & $\mathrm{AIC}$ & $\mathrm{BIC}$ \\
\hline 1 & 1 & 0.042 & -0.127 & 1.237 & 0.01 & -2704.6 & 5415.3 & 5431.7 \\
1 & 1 & 0.853 & -0.924 & 1.216 & 0.05 & -2699.8 & 5405.7 & 5422.1 \\
1 & 1 & 0.636 & -0.792 & 1.014 & 0.2 & -2691.1 & 5388.1 & 5404.6 \\
1 & 1 & 0.622 & -0.800 & 0.909 & 0.3 & -2691.2 & 5388.4 & 5404.8 \\
1 & 1 & 0.603 & -0.866 & 0.682 & 0.5 & -2691.7 & 5389.3 & 5405.7 \\
1 & 1 & 0.325 & -2.069 & 0.085 & 1 & -2713.3 & 5432.6 & 5449 \\
\hline \hline
\end{tabular}

Table F.10: Univariate model sensitivity analysis: parameter estimates for selected values of $R$. Stock:XOM

Table features univariate model parameter estimates for selected values of the measurement noise variance $R$. The model's likelihood as well as the AIC and $\mathrm{BIC}$ fit criteria are presented. 


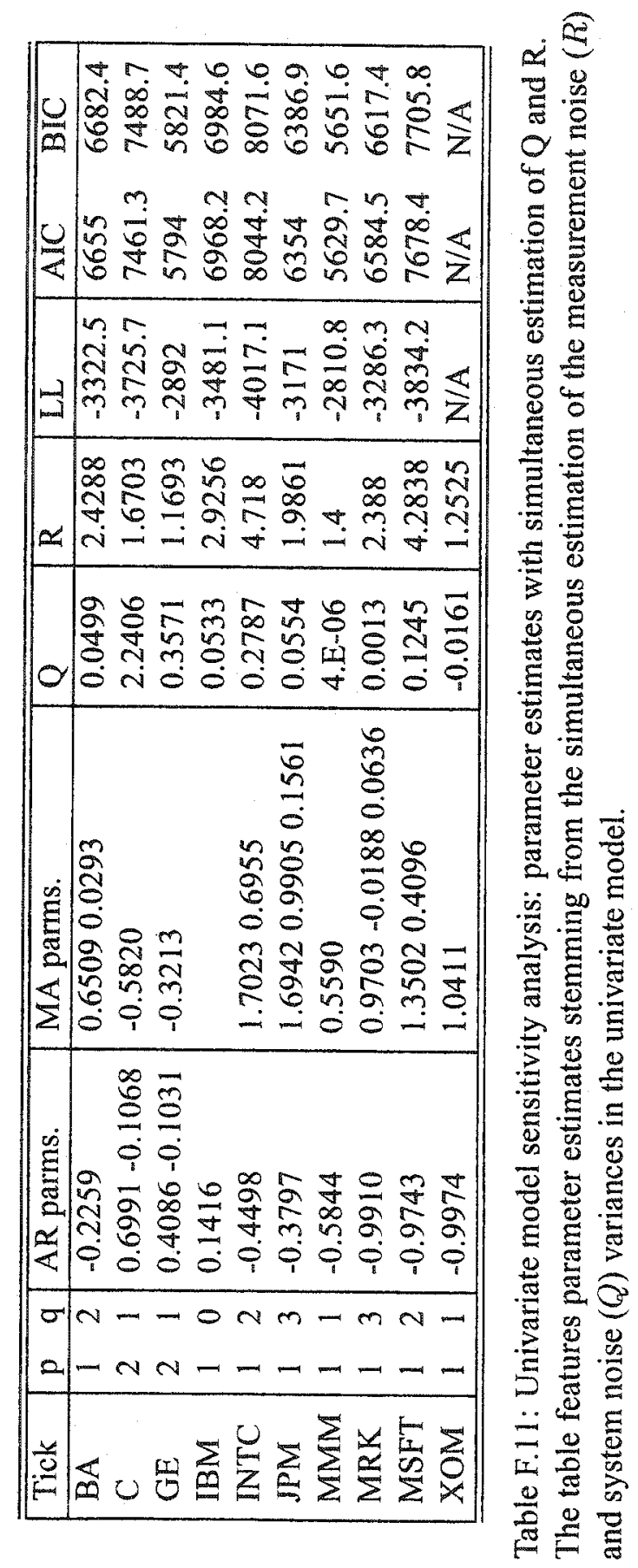


Appendix G

Summary Tables 


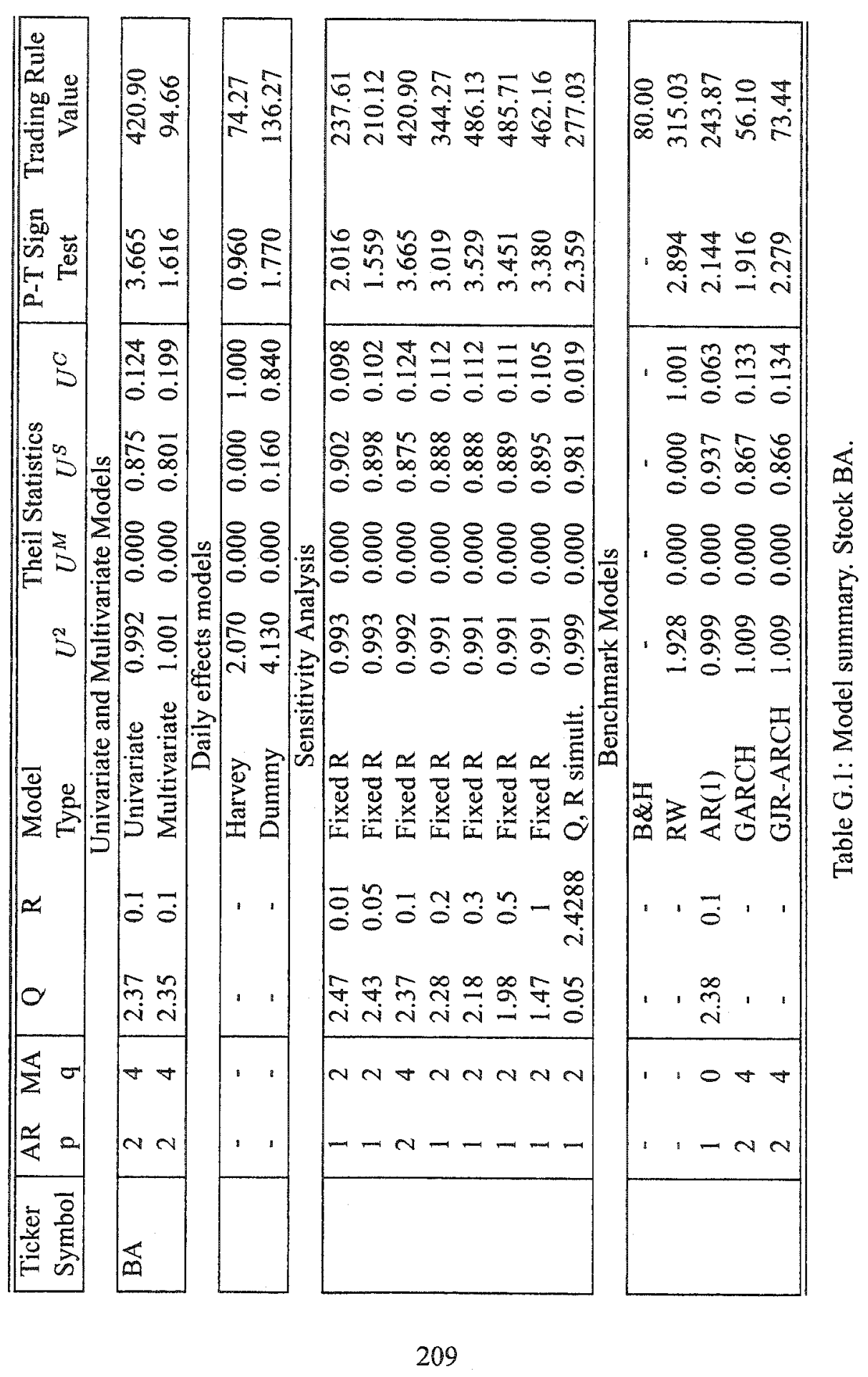




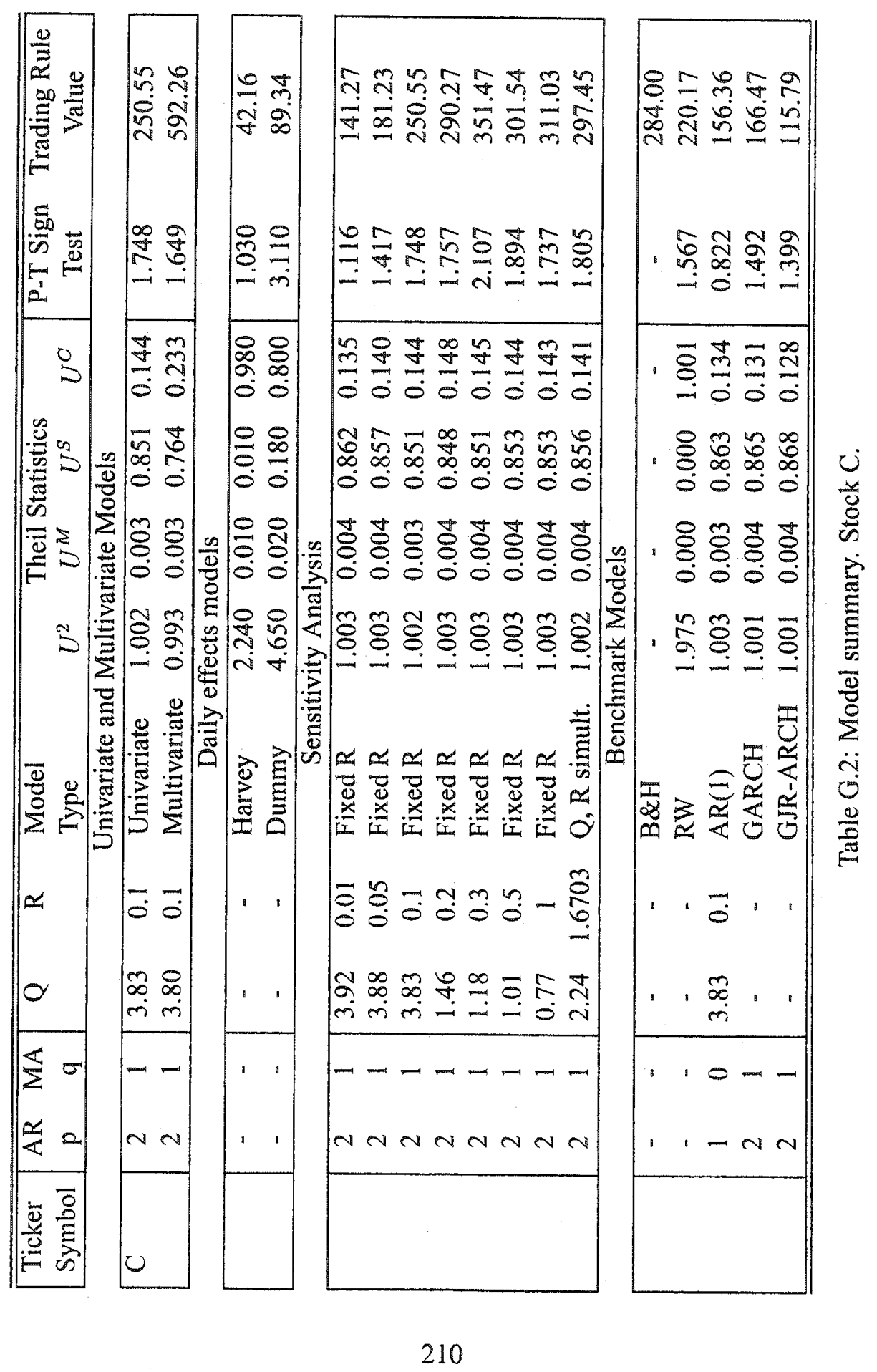




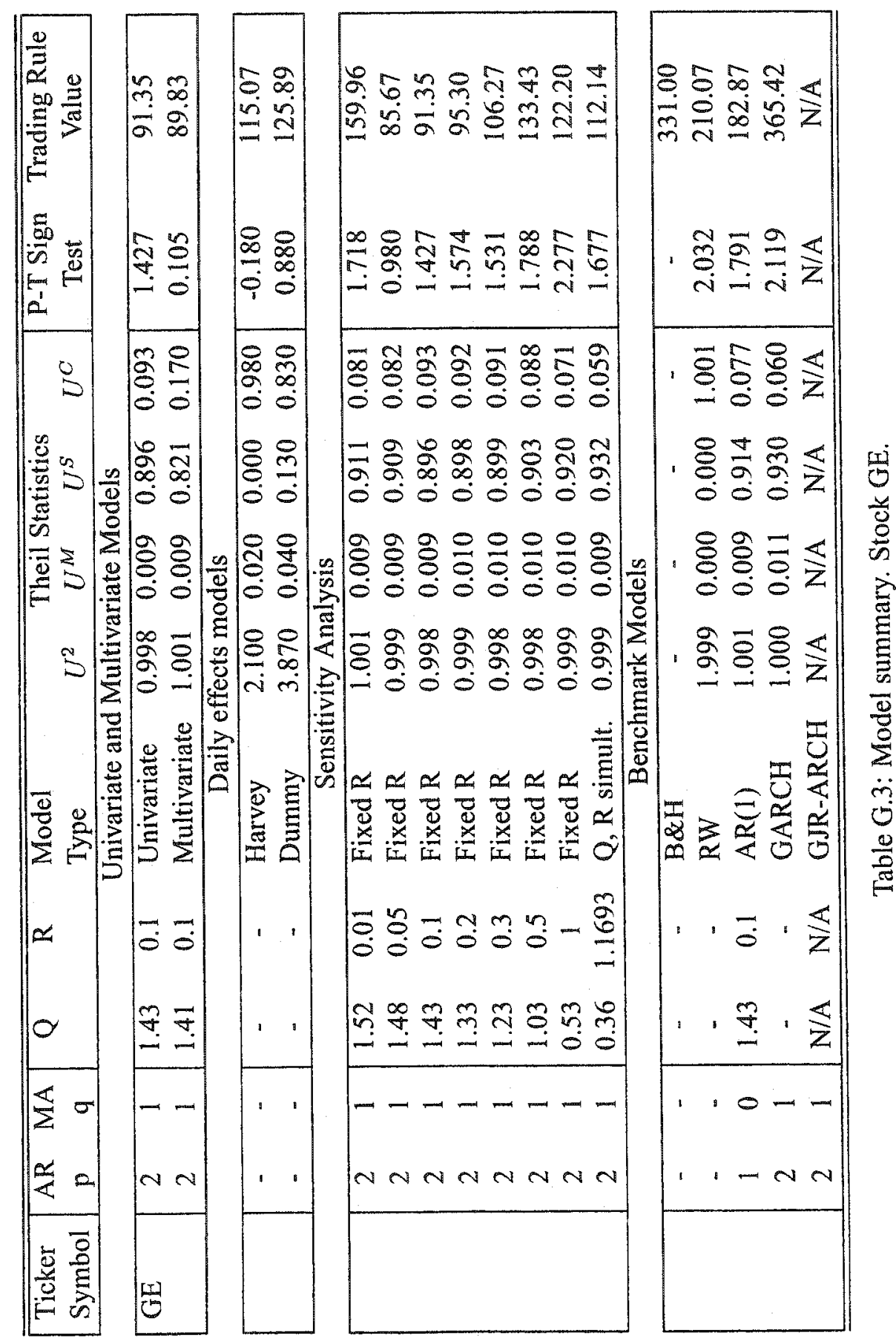




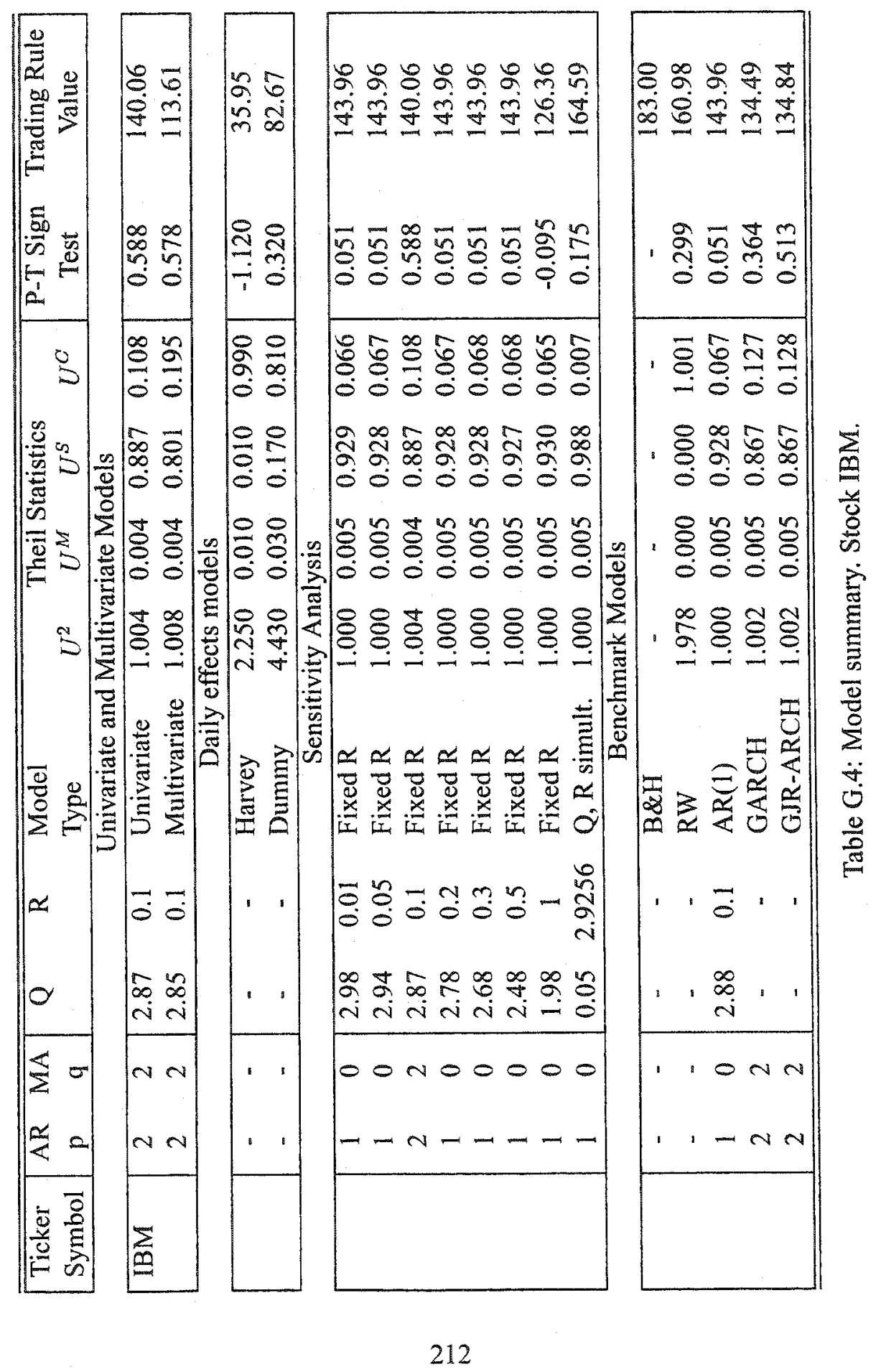




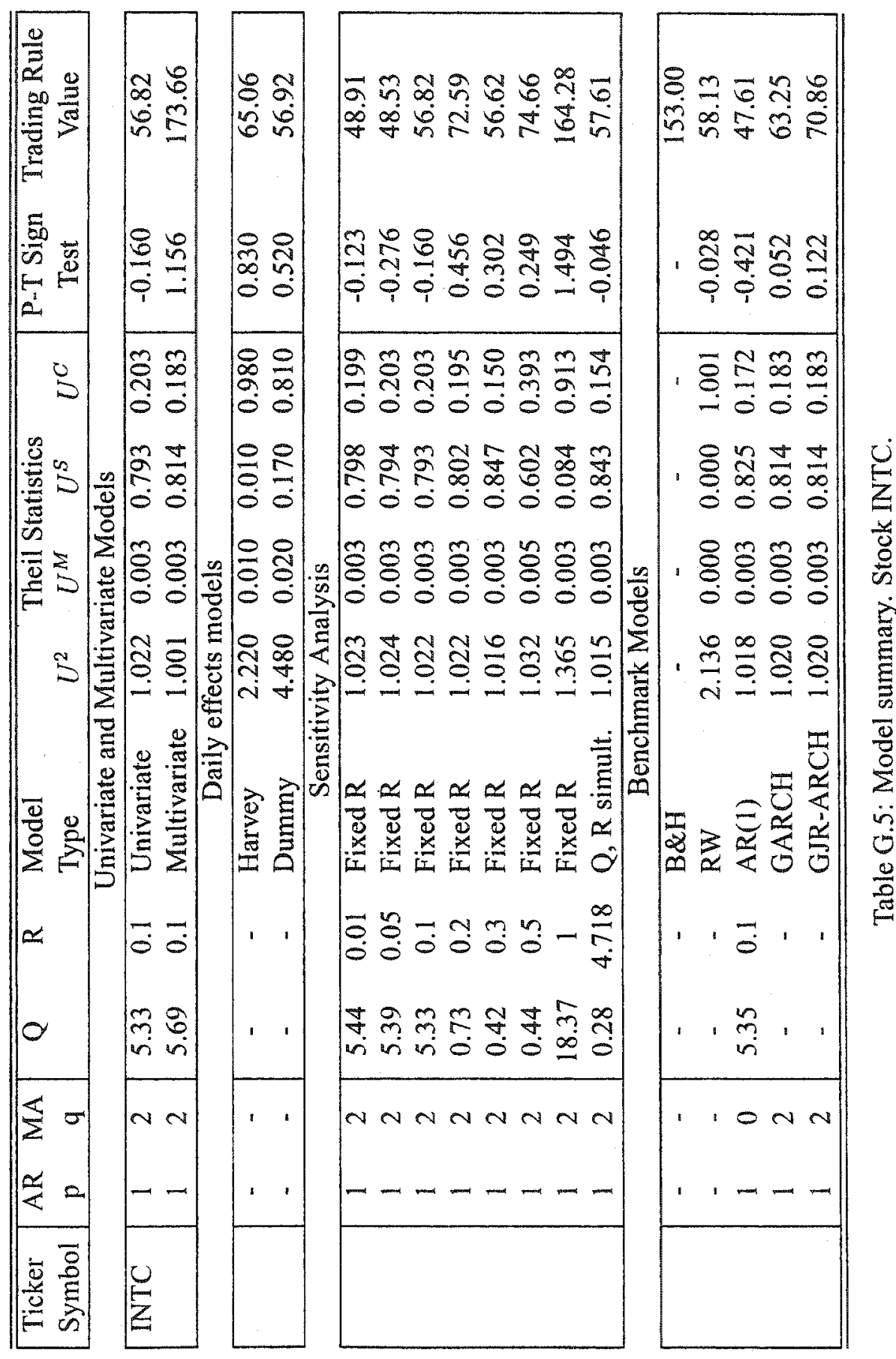




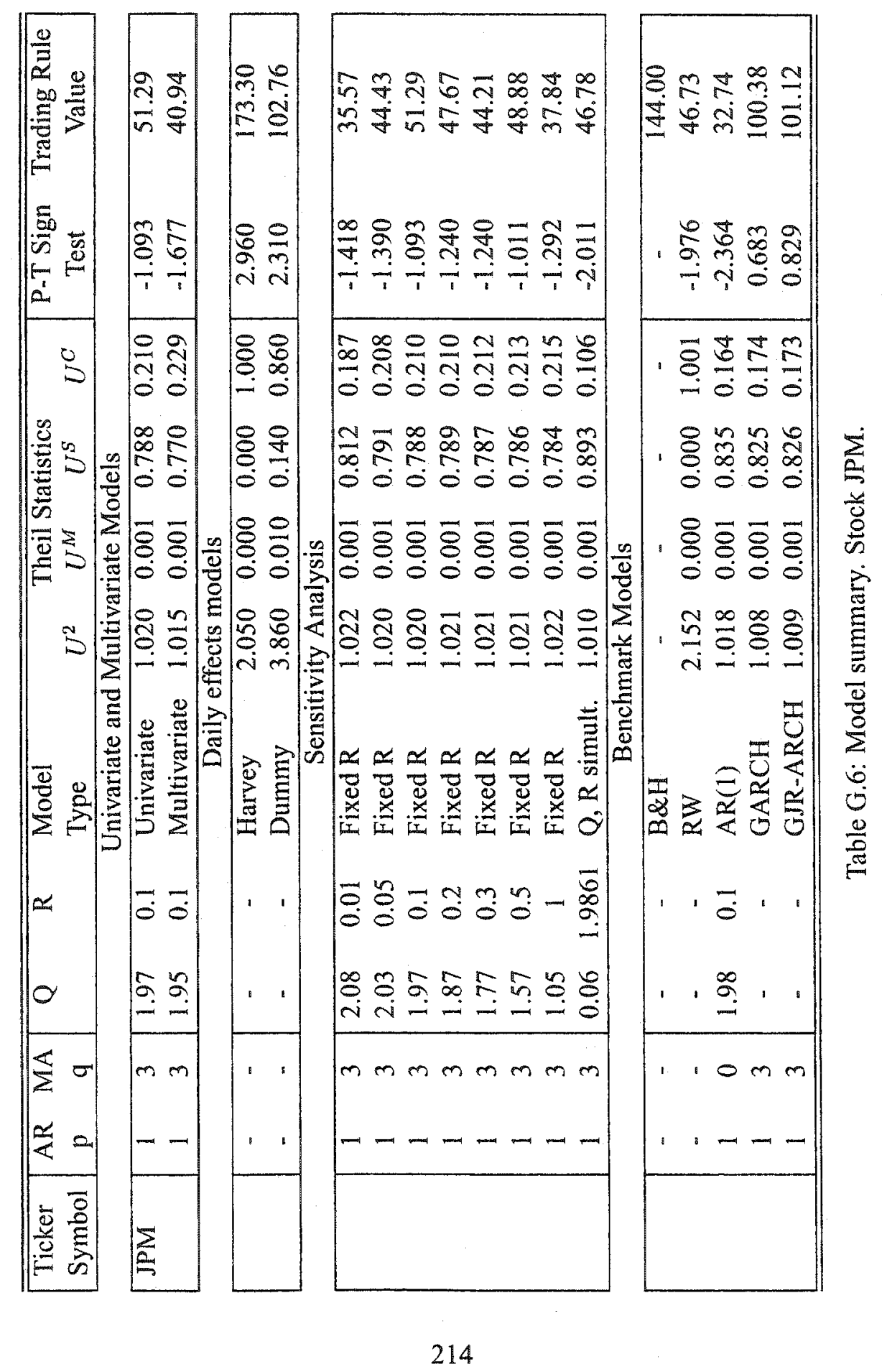




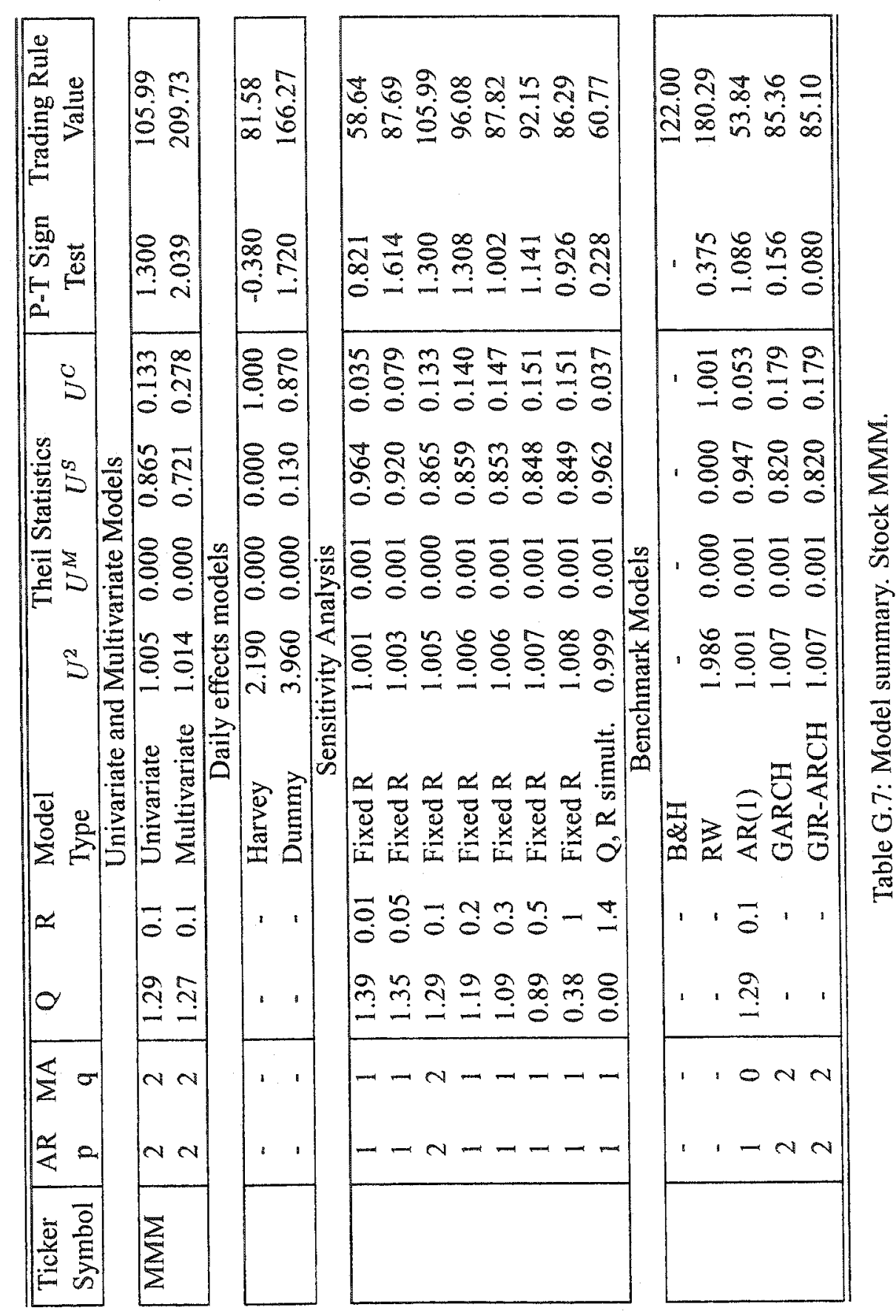




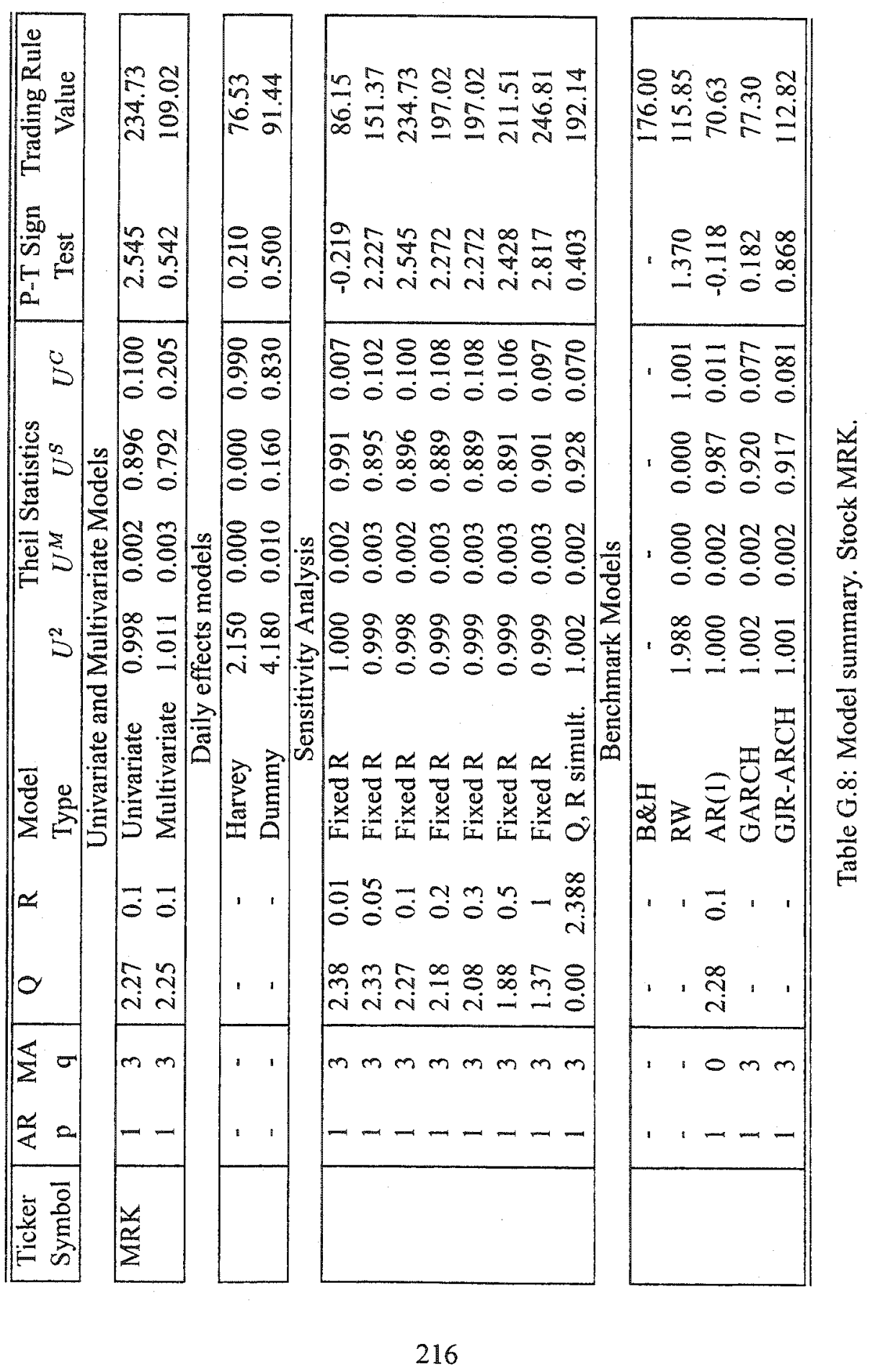




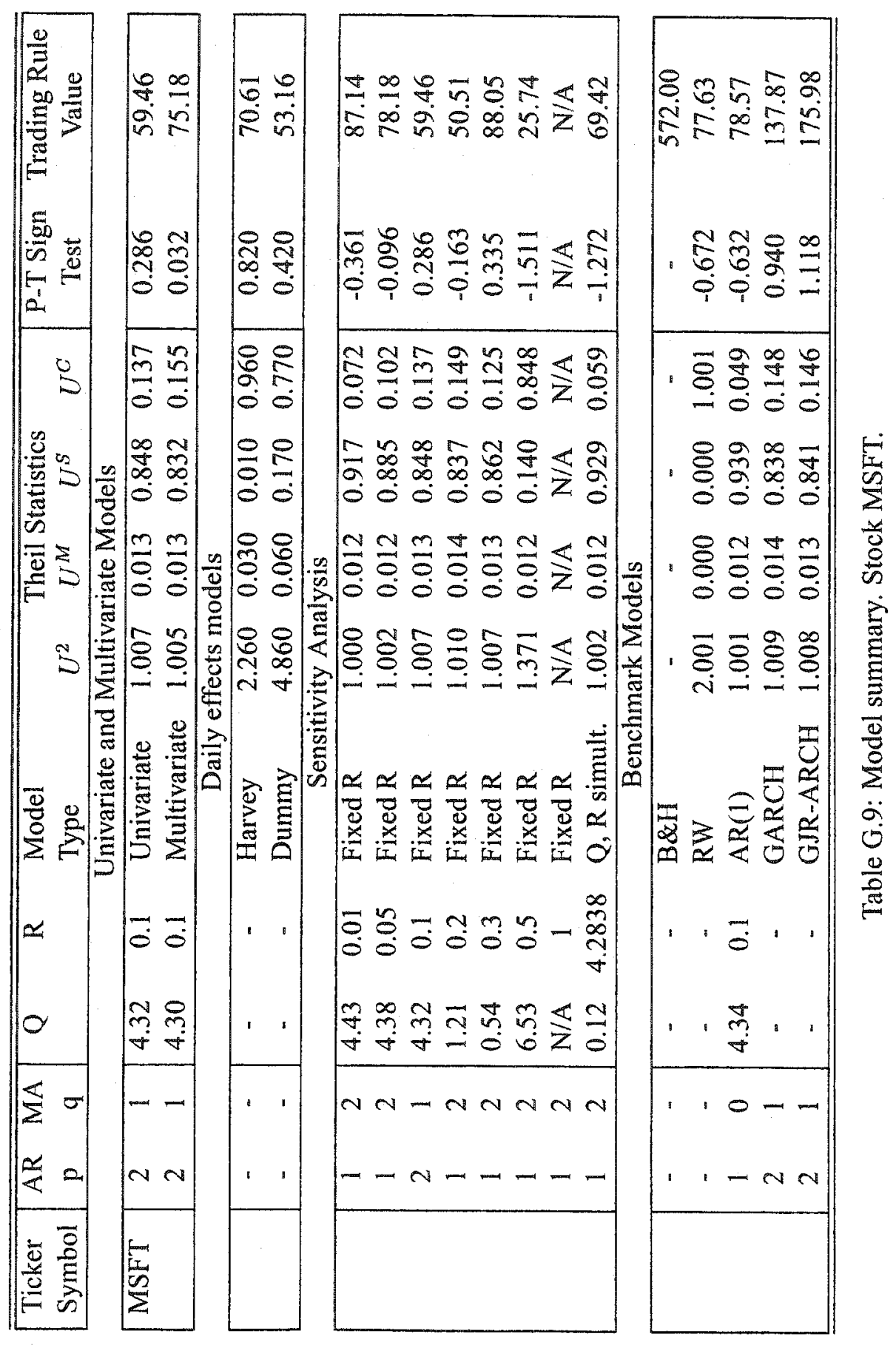




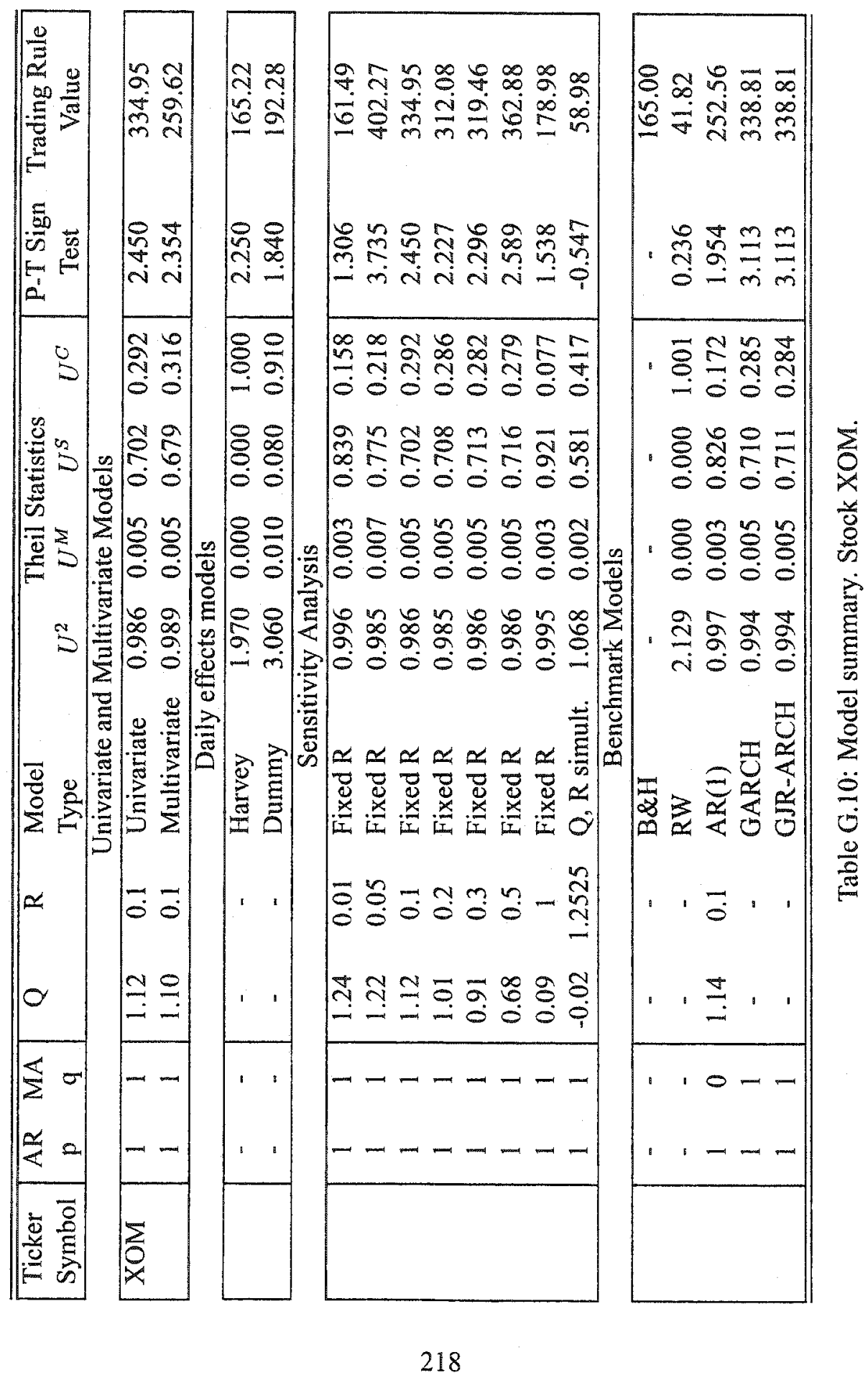




\section{Appendix $\mathbf{H}$}

\section{Time Varying Parameter Univariate Models}

\begin{tabular}{|c|c|c|c|c|c|c|}
\hline \multirow{2}{*}{$\begin{array}{l}\text { Ticker } \\
\text { Symbol }\end{array}$} & \multicolumn{4}{|c|}{ Theil Statistics } & \multirow{2}{*}{$\begin{array}{c}\text { P-T Sign } \\
\text { Test }\end{array}$} & \multirow{2}{*}{$\begin{array}{c}\text { Trading Rule } \\
\text { Value }\end{array}$} \\
\hline & $U^{2}$ & $U^{M}$ & $U^{S}$ & $U^{C}$ & & \\
\hline$\overline{\mathrm{BA}}$ & 1.284 & 0.001 & 0.222 & 0.778 & 2.305 & 67.877 \\
\hline $\mathrm{C}$ & 1.244 & 0.003 & 0.256 & 0.741 & 1.355 & 45.524 \\
\hline GE & 1.149 & 0.010 & 0.407 & 0.582 & -0.592 & 35.504 \\
\hline IBM & 1.113 & 0.003 & 0.388 & 0.608 & 0.919 & 84.496 \\
\hline INTC & 1.094 & 0.003 & 0.436 & 0.561 & 1.256 & 220.918 \\
\hline JPM & 1.108 & 0.001 & 0.463 & 0.536 & 0.895 & 37.297 \\
\hline MMM & 1.122 & 0.000 & 0.339 & 0.660 & 0.638 & 142.166 \\
\hline MRK & 1.129 & 0.004 & 0.386 & 0.610 & 0.692 & 66.414 \\
\hline MSFT & 1.160 & 0.006 & 0.367 & 0.628 & -1.231 & 46.217 \\
\hline XOM & 1.063 & 0.003 & 0.536 & 0.462 & -0.474 & 93.727 \\
\hline
\end{tabular}

Table H.1: Prediction results from the time varying univariate model with estimation window size of 20 observations. 


\begin{tabular}{|l|cccc|c|c|}
\hline \hline $\begin{array}{l}\text { Ticker } \\
\text { Symbol }\end{array}$ & $U^{2}$ & $U^{M}$ & $U^{S}$ & $U^{C}$ & P-T Sign & Trading Rule \\
Test & Value \\
\hline \hline BA & 1.185 & 0.000 & 0.352 & 0.648 & -0.181 & 28.075 \\
C & 1.157 & 0.003 & 0.382 & 0.615 & 1.051 & 31.699 \\
GE & 1.109 & 0.011 & 0.518 & 0.471 & 0.907 & 61.085 \\
IBM & 1.073 & 0.005 & 0.485 & 0.510 & 1.461 & 59.336 \\
INTC & 1.061 & 0.002 & 0.547 & 0.451 & -0.020 & 112.640 \\
JPM & 1.095 & 0.002 & 0.450 & 0.548 & 1.571 & 66.292 \\
MMM & 1.098 & 0.001 & 0.424 & 0.576 & 0.816 & 131.430 \\
MRK & 1.090 & 0.005 & 0.477 & 0.519 & 0.657 & 54.658 \\
MSFT & 1.087 & 0.008 & 0.508 & 0.484 & -2.121 & 32.166 \\
XOM & 1.031 & 0.003 & 0.608 & 0.389 & 0.671 & 134.771 \\
\hline \hline
\end{tabular}

Table H.2: Prediction results from the time varying univariate model with estimation window size of 30 observations.

\begin{tabular}{|c|c|c|c|c|c|c|}
\hline \multirow{2}{*}{$\begin{array}{l}\text { Ticker } \\
\text { Symbol }\end{array}$} & \multicolumn{4}{|c|}{ Theil Statistics } & \multirow{2}{*}{$\begin{array}{l}\text { P-T Sign } \\
\text { Test }\end{array}$} & \multirow{2}{*}{$\begin{array}{c}\text { Trading Rule } \\
\text { Value }\end{array}$} \\
\hline & $U^{2}$ & $U^{M}$ & $U^{S}$ & $U^{C}$ & & \\
\hline$\overline{\mathrm{BA}}$ & 1.101 & 0.000 & 0.472 & 0.528 & -0.592 & 55.825 \\
\hline $\mathrm{C}$ & 1.072 & 0.004 & 0.584 & 0.412 & 0.365 & 38.824 \\
\hline $\mathrm{GE}$ & 1.062 & 0.011 & 0.622 & 0.367 & -0.199 & 40.600 \\
\hline IBM & 1.054 & 0.003 & 0.585 & 0.411 & 1.159 & 82.190 \\
\hline INTC & 1.028 & 0.003 & 0.609 & 0.388 & 1.931 & 506.270 \\
\hline JPM & 1.058 & 0.002 & 0.512 & 0.486 & 2.880 & 144.416 \\
\hline MMM & 1.076 & 0.000 & 0.535 & 0.464 & 0.558 & 94.482 \\
\hline MRK & 1.054 & 0.004 & 0.571 & 0.425 & 1.341 & 74.003 \\
\hline MSFT & 1.055 & 0.009 & 0.616 & 0.375 & -3.179 & 16.848 \\
\hline XOM & 1.011 & 0.004 & 0.653 & 0.343 & 1.292 & 155.014 \\
\hline
\end{tabular}

Table H.3: Prediction results from the time varying univariate model with estimation window size of 50 observations. 


\begin{tabular}{|c|c|c|c|c|c|c|}
\hline \multirow{2}{*}{$\begin{array}{l}\text { Ticker } \\
\text { Symbol }\end{array}$} & \multicolumn{4}{|c|}{ Theil Statistics } & \multirow{2}{*}{$\begin{array}{l}\text { P-T Sign } \\
\text { Test }\end{array}$} & \multirow{2}{*}{$\begin{array}{c}\text { Trading Rule } \\
\text { Value }\end{array}$} \\
\hline & $U^{2}$ & $U^{M}$ & $U^{S}$ & $U^{C}$ & & \\
\hline$\overline{\mathrm{BA}}$ & 1.046 & 0.000 & 0.619 & 0.381 & 1.206 & 95.975 \\
\hline C & 1.043 & 0.004 & 0.707 & 0.289 & 1.074 & 56.085 \\
\hline GE & 1.034 & 0.009 & 0.753 & 0.237 & 0.307 & 83.494 \\
\hline $\mathrm{IBM}$ & 1.027 & 0.005 & 0.686 & 0.309 & 0.651 & 49.645 \\
\hline INTC & 1.010 & 0.003 & 0.683 & 0.314 & 2.731 & 510.804 \\
\hline JPM & 1.044 & 0.001 & 0.618 & 0.381 & 1.876 & 91.600 \\
\hline MMM & 1.032 & 0.001 & 0.698 & 0.301 & 1.089 & 106.256 \\
\hline MRK & 1.033 & 0.003 & 0.689 & 0.308 & 1.053 & 76.086 \\
\hline MSFT & 1.031 & 0.012 & 0.755 & 0.234 & -1.244 & 40.092 \\
\hline XOM & 1.001 & 0.005 & 0.716 & 0.280 & 2.142 & 143.683 \\
\hline
\end{tabular}

Table H.4: Prediction results from the time varying univariate model with estimation window size of 100 observations.

\begin{tabular}{|c|c|c|c|c|c|c|}
\hline \multirow{2}{*}{$\begin{array}{l}\text { Ticker } \\
\text { Symbol }\end{array}$} & \multicolumn{4}{|c|}{ Theil Statistics } & \multirow{2}{*}{$\begin{array}{c}\text { P-T Sign } \\
\text { Test }\end{array}$} & \multirow{2}{*}{$\begin{array}{c}\text { Trading Rule } \\
\text { Value }\end{array}$} \\
\hline & $U^{2}$ & $U^{M}$ & $U^{S}$ & $U^{C}$ & & \\
\hline$\overline{\mathrm{BA}}$ & 1.011 & 0.000 & 0.722 & $0 . \overline{278}$ & 1.053 & 158.413 \\
\hline $\mathrm{C}$ & 1.019 & 0.004 & 0.804 & 0.192 & 0.908 & 22.035 \\
\hline $\mathrm{GE}$ & 1.013 & 0.010 & 0.822 & 0.168 & 1.200 & 85.441 \\
\hline IBM & 1.012 & 0.006 & 0.755 & 0.239 & 1.774 & 118.024 \\
\hline INTC & 1.009 & 0.003 & 0.746 & 0.251 & 2.865 & 669.861 \\
\hline JPM & 1.020 & 0.001 & 0.731 & 0.268 & 0.558 & 47.078 \\
\hline $\mathrm{MMM}$ & 1.036 & 0.001 & 0.679 & 0.320 & 0.679 & 83.675 \\
\hline MRK & 1.015 & 0.003 & 0.800 & 0.197 & 0.872 & 102.493 \\
\hline MSFT & 1.014 & 0.013 & 0.853 & 0.134 & -1.162 & 38.550 \\
\hline XOM & 0.996 & 0.008 & 0.701 & 0.291 & 2.608 & 380.744 \\
\hline
\end{tabular}

Table H.5: Prediction results from the time varying univariate model with estimation window size of 200 observations. 


\begin{tabular}{|c|c|c|c|c|c|c|}
\hline \multirow{2}{*}{$\begin{array}{l}\text { Ticker } \\
\text { Symbol }\end{array}$} & \multicolumn{4}{|c|}{ Theil Statistics } & \multirow{2}{*}{$\begin{array}{l}\text { P-T Sign } \\
\text { Test }\end{array}$} & \multirow{2}{*}{$\begin{array}{c}\text { Trading Rule } \\
\text { Value }\end{array}$} \\
\hline & $U^{2}$ & $U^{M}$ & $U^{S}$ & $U^{C}$ & & \\
\hline$\overline{\mathrm{BA}}$ & 1.083 & 0.000 & 0.545 & $\overline{0.455}$ & 0.843 & 64.356 \\
\hline $\mathrm{C}$ & 1.072 & 0.004 & 0.597 & 0.399 & 1.586 & 61.838 \\
\hline $\mathrm{GE}$ & 1.054 & 0.011 & 0.680 & 0.310 & 0.627 & 57.097 \\
\hline IBM & 1.035 & 0.004 & 0.634 & 0.362 & 1.961 & 123.736 \\
\hline INTC & 1.025 & 0.003 & 0.651 & 0.346 & 1.719 & 336.096 \\
\hline JPM & 1.044 & 0.001 & 0.621 & 0.377 & 2.433 & 106.649 \\
\hline $\mathrm{MMM}$ & 1.041 & 0.001 & 0.619 & 0.381 & 0.555 & 155.231 \\
\hline MRK & 1.041 & 0.004 & 0.644 & 0.352 & 1.117 & 91.975 \\
\hline MSFT & 1.049 & 0.009 & 0.665 & 0.326 & -1.152 & 55.814 \\
\hline XOM & 1.006 & 0.004 & 0.714 & 0.282 & 1.107 & 172.516 \\
\hline
\end{tabular}

Table H.6: Prediction results from the "average" time varying univariate model. 


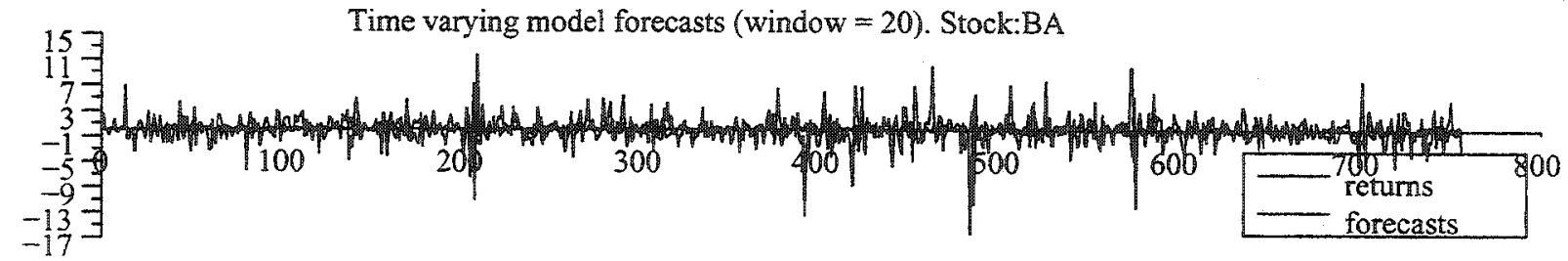

Time varying model forecasts (window $=30$ ). Stock: $\mathrm{BA}$

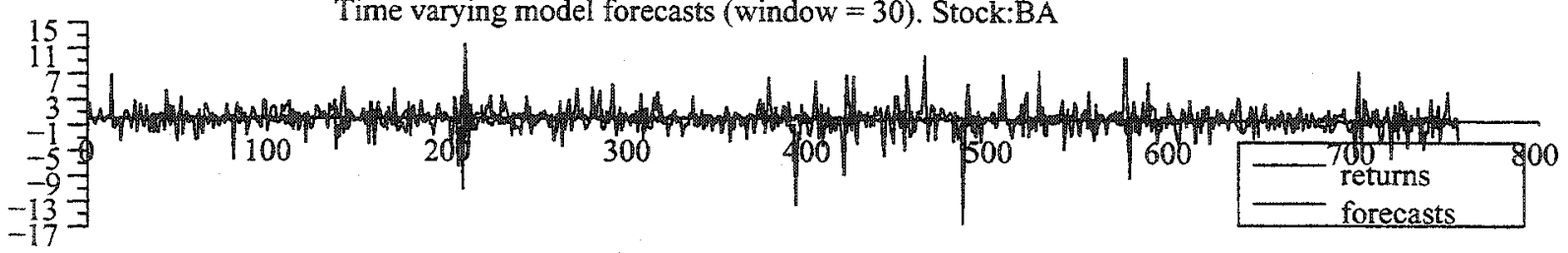

Time varying model forecasts (window $=50$ ). Stock:BA

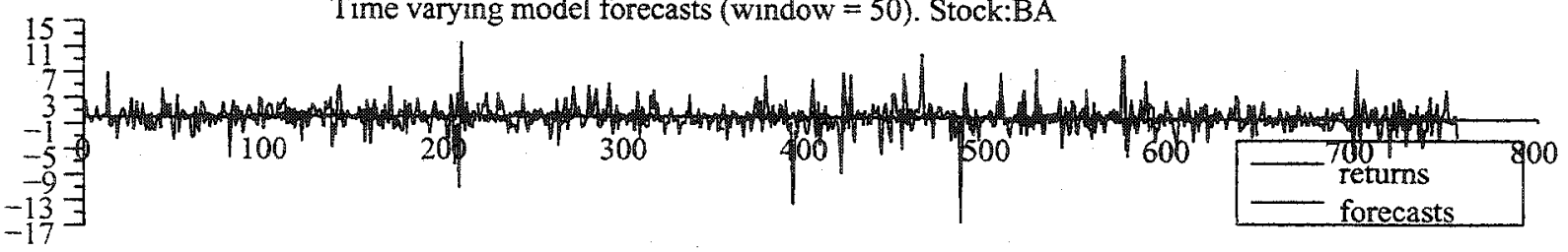

Time varying model forecasts (window $=100$ ). Stock: $\mathrm{BA}$

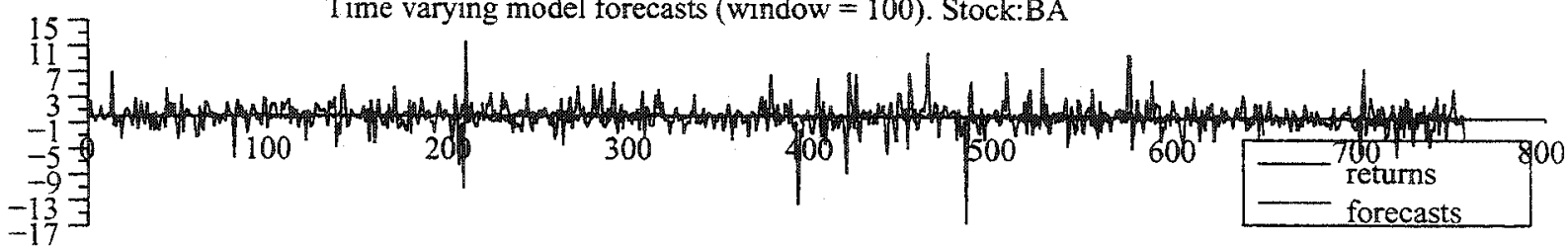

Time varying model forecasts (window $=200$ ). Stock: $B A$

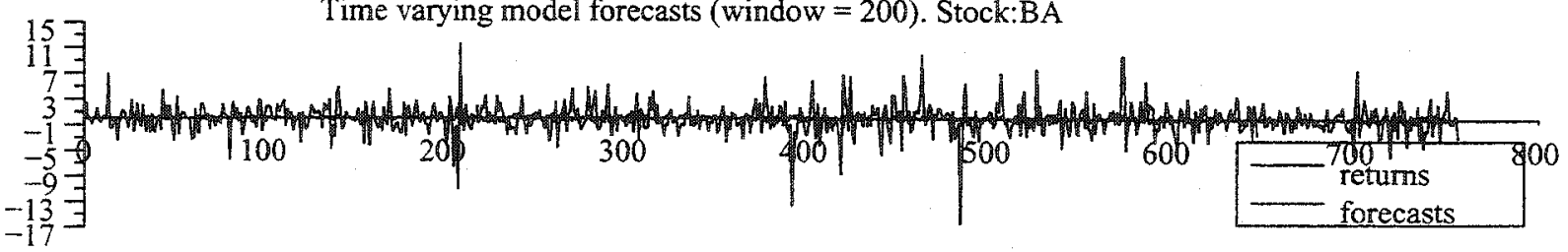

Figure H.1: Time varying parameter model forecasts for selected estimation window sizes. From top to bottom, window sizes are respectively, 20, 30, 50, 100 and 200 measurements. Stock: BA 


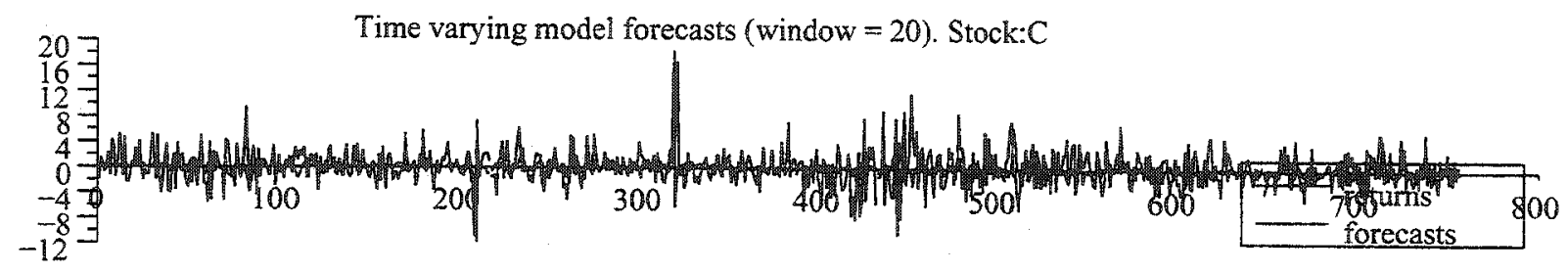

Time varying model forecasts (window $=30$ ). Stock: $\mathrm{C}$
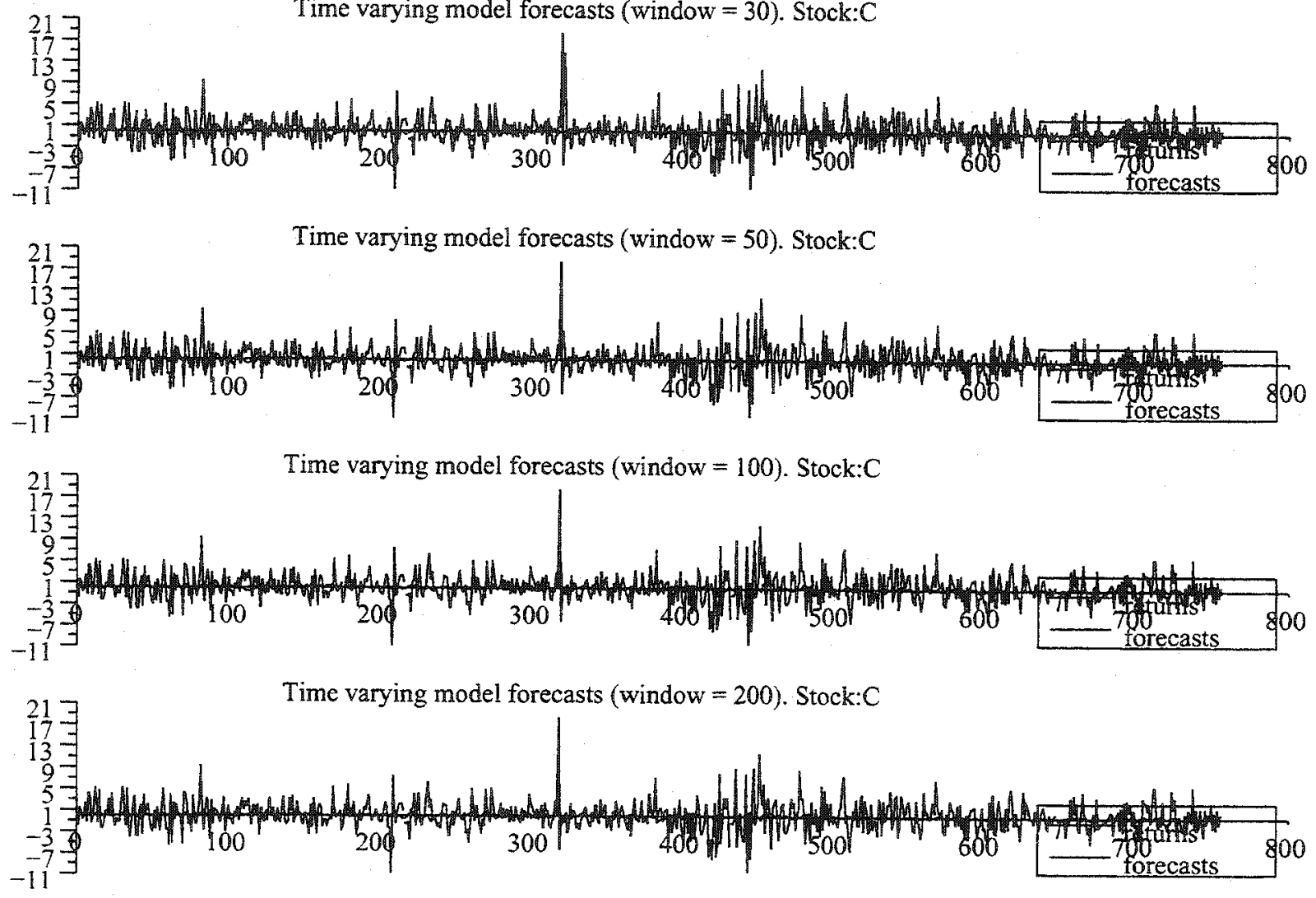

Figure H.2: Time varying parameter model forecasts for selected estimation window sizes. From top to bottom, window sizes are respectively, 20, 30, 50, 100 and 200 measurements. Stock: C 

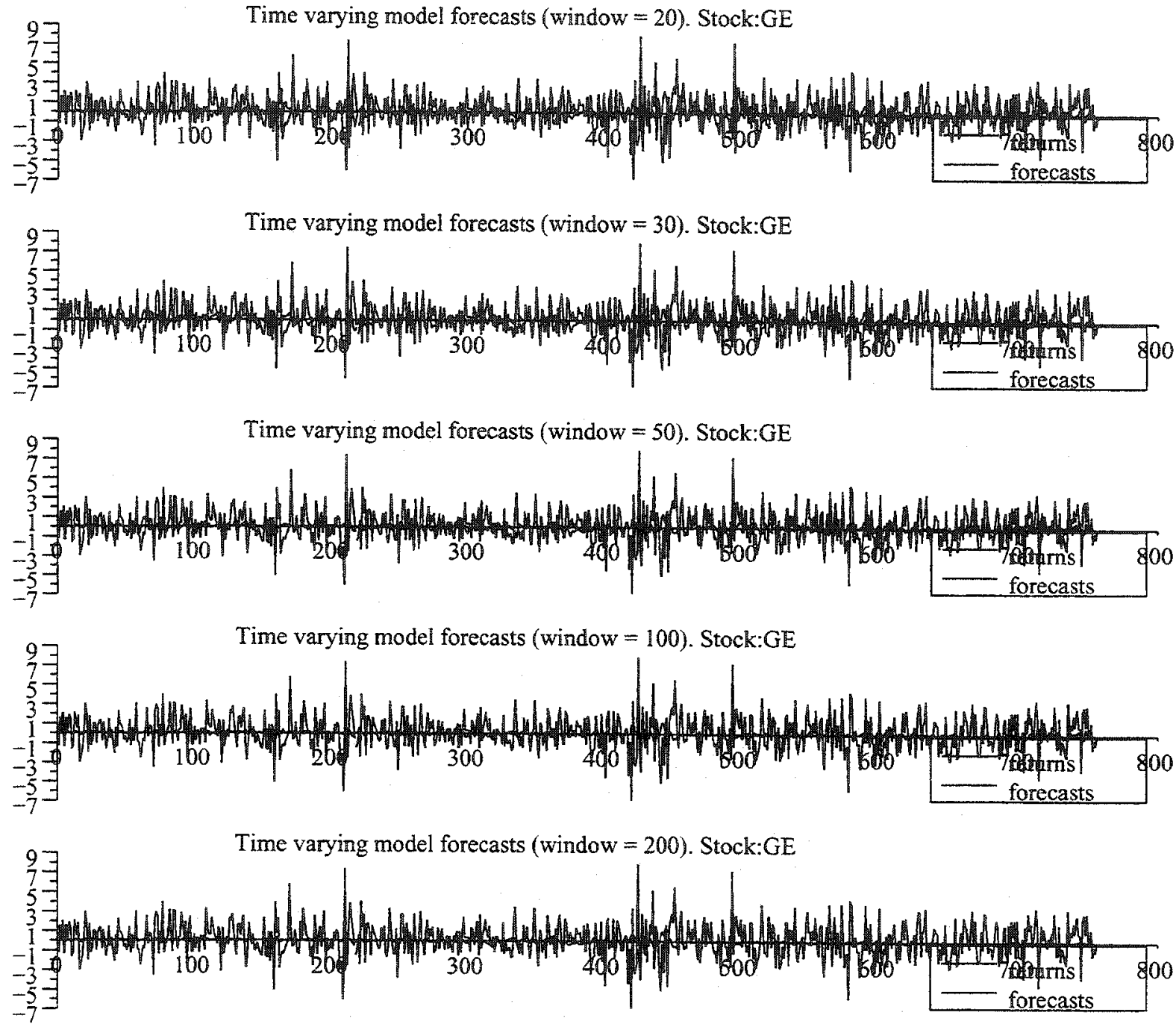

Figure H.3: Time varying parameter model forecasts for selected estimation window sizes. From top to bottom, window sizes are respectively, 20, 30, 50, 100 and 200 measurements. Stock: GE 


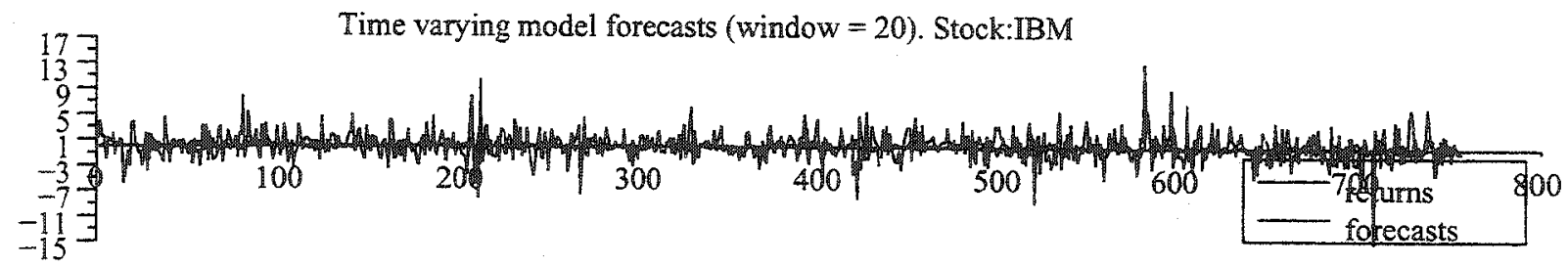

Time varying model forecasts (window $=30$ ). Stock:IBM
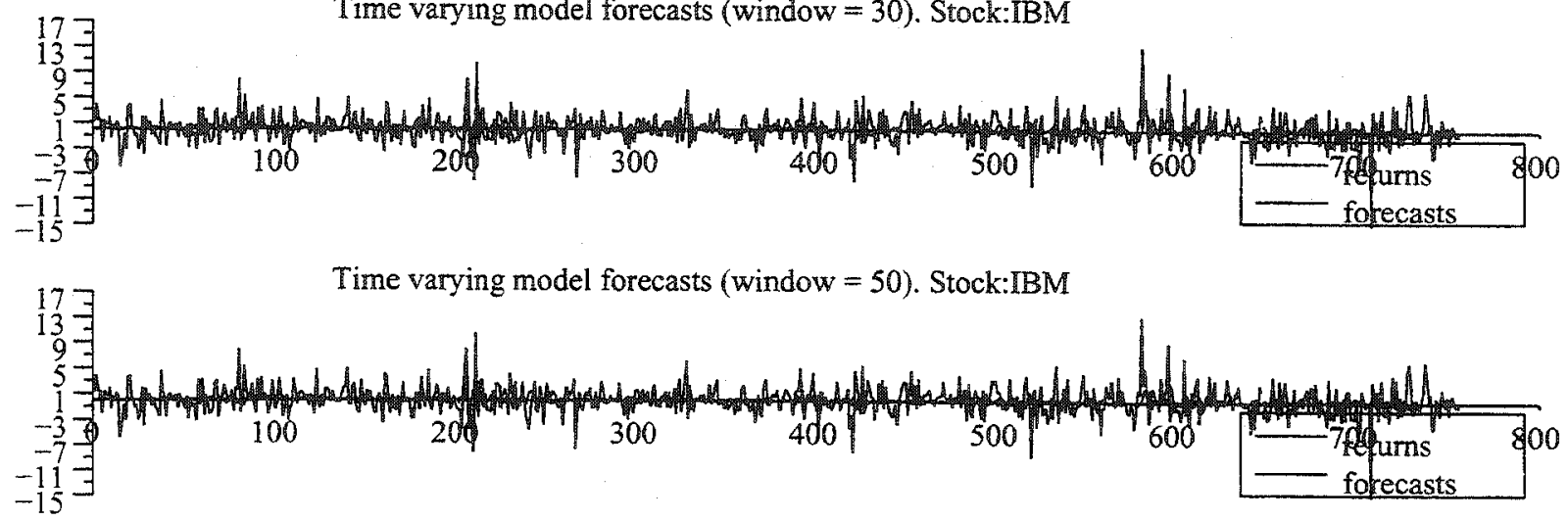

Time varying model forecasts $($ window $=100$ ). Stock:IBM

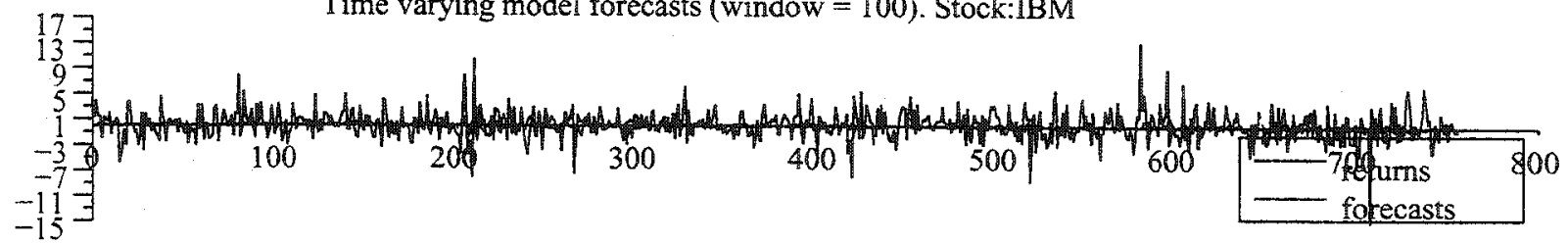

Time varying model forecasts (window $=200$ ). Stock:IBM

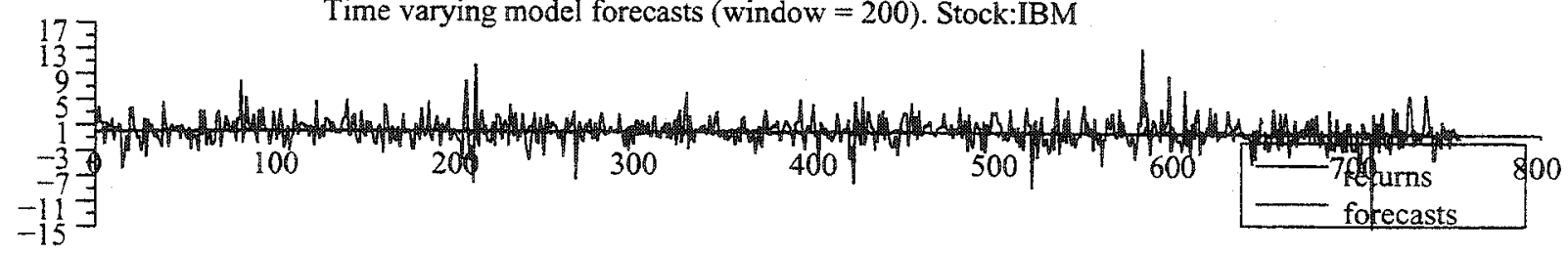

Figure H.4: Time varying parameter model forecasts for selected estimation window sizes. From top to bottom, window sizes are respectively, 20, 30, 50, 100 and 200 measurements. Stock: IBM 

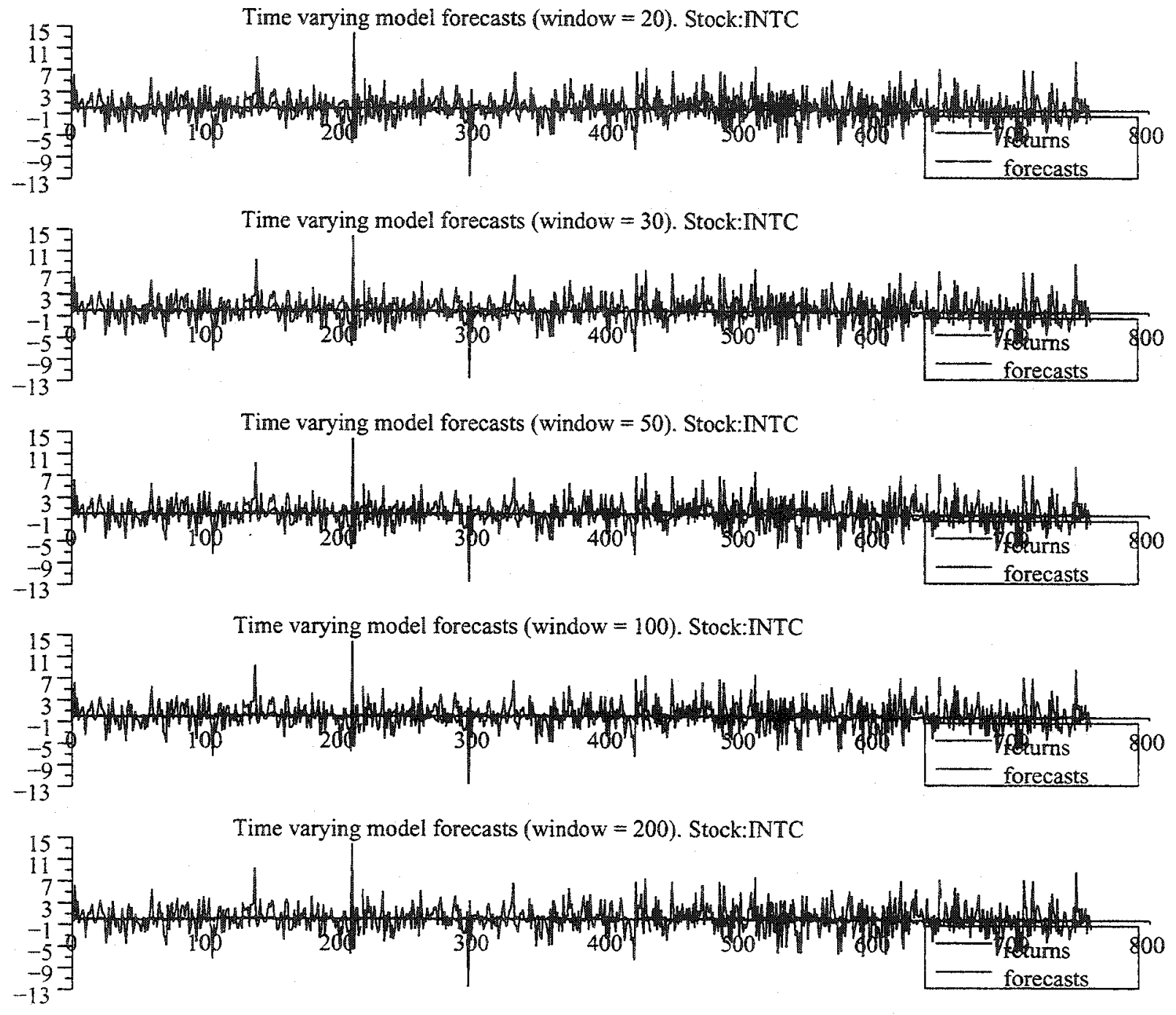

Figure H.5: Time varying parameter model forecasts for selected estimation window sizes. From top to bottom, window sizes are respectively, 20, 30, 50, 100 and 200 measurements. Stock: INTC 

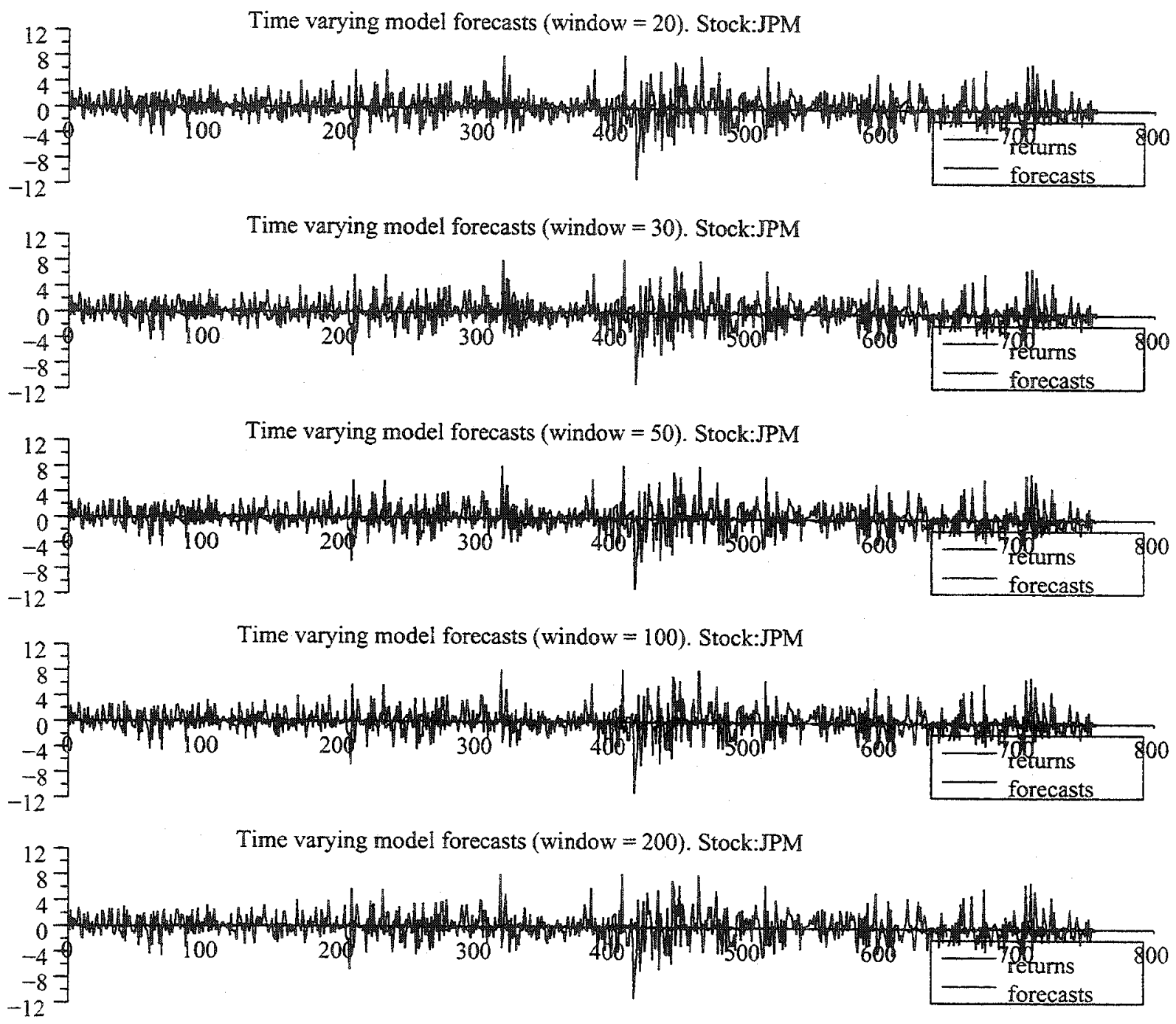

Figure H.6: Time varying parameter model forecasts for selected estimation window sizes. From top to bottom, window sizes are respectively, 20, 30, 50, 100 and 200 measurements. Stock: JPM 

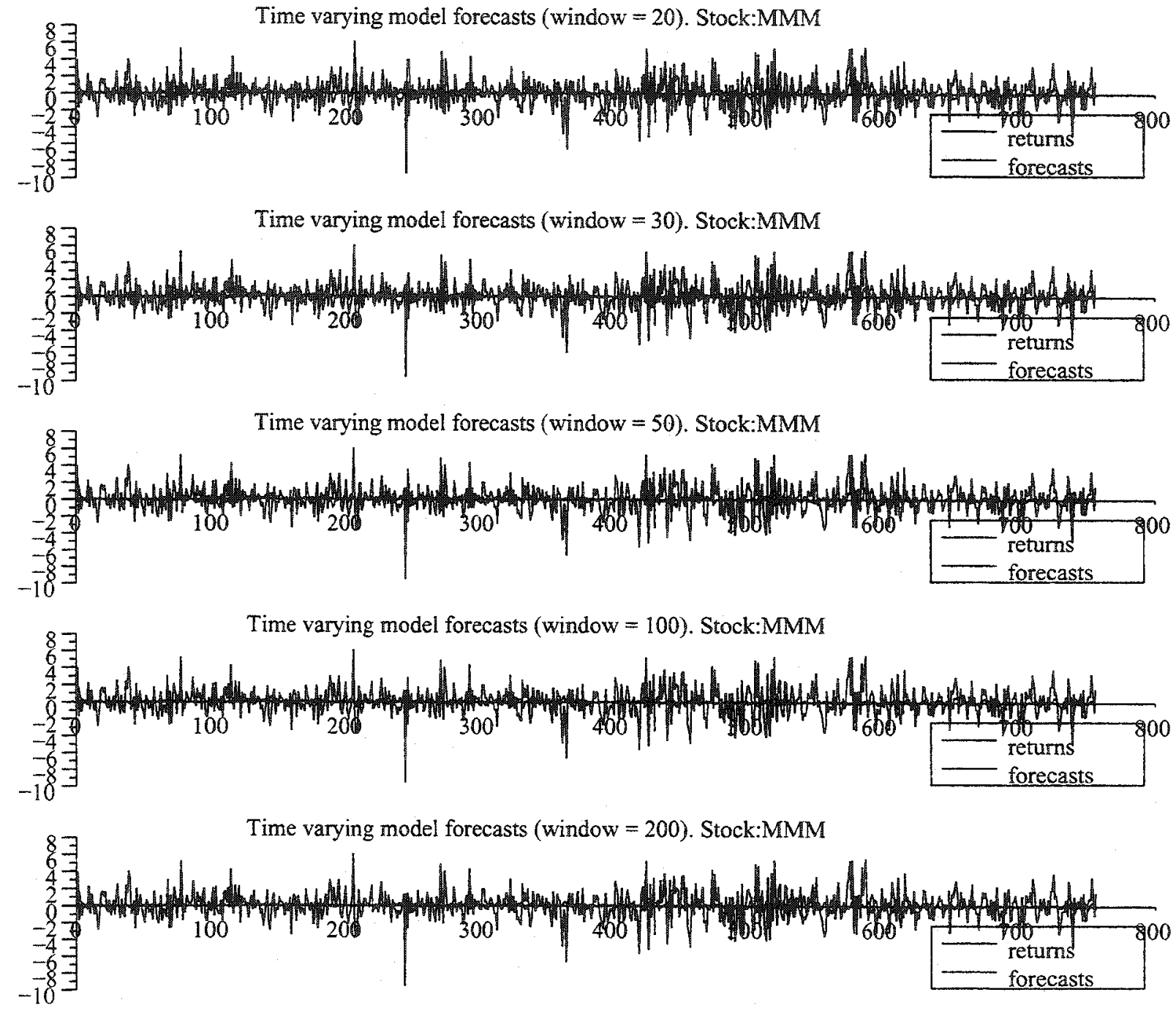

Figure H.7: Time varying parameter model forecasts for selected estimation window sizes. From top to bottom, window sizes are respectively, 20, 30, 50, 100 and 200 measurements. Stock: MMM 

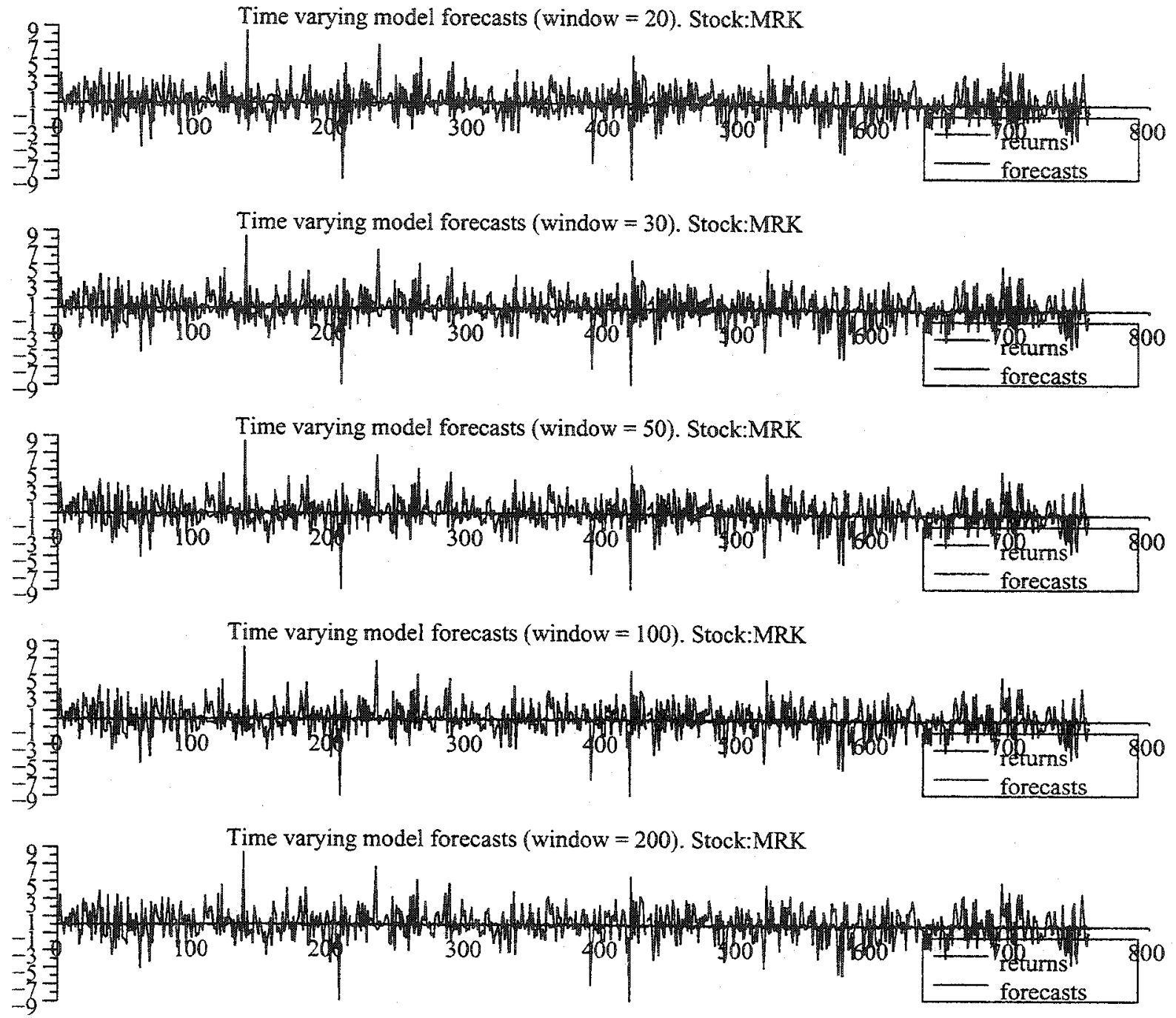

Figure H.8: Time varying parameter model forecasts for selected estimation window sizes. From top to bottom, window sizes are respectively, 20, 30, 50, 100 and 200 measurements. Stock: MRK 

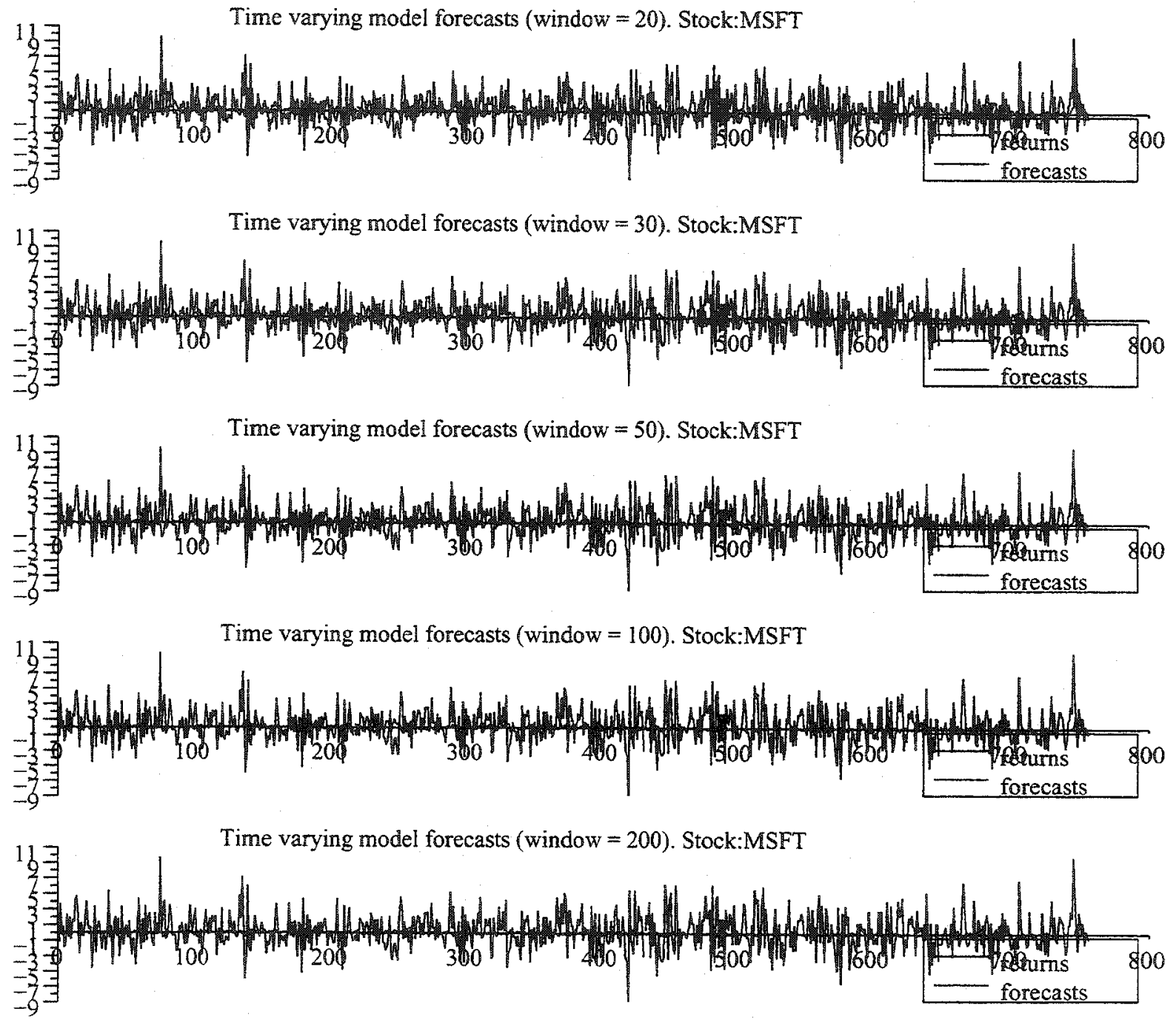

Figure H.9: Time varying parameter model forecasts for selected estimation window sizes. From top to bottom, window sizes are respectively, 20, 30, 50, 100 and 200 measurements. Stock: MSFT 


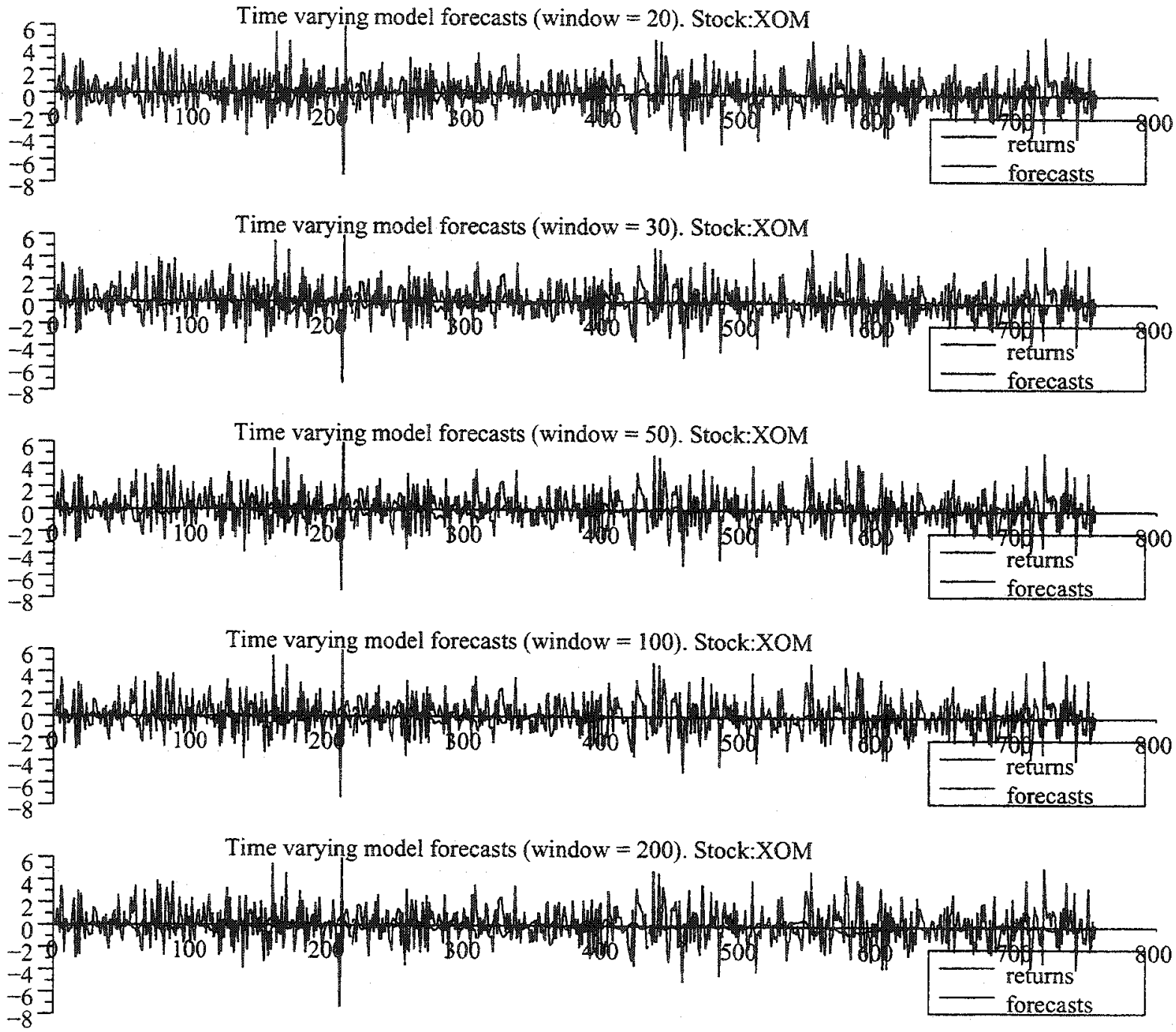

Figure H.10: Time varying parameter model forecasts for selected estimation window sizes. From top to bottom, window sizes are respectively, 20, 30, 50, 100 and 200 measurements. Stock: XOM 

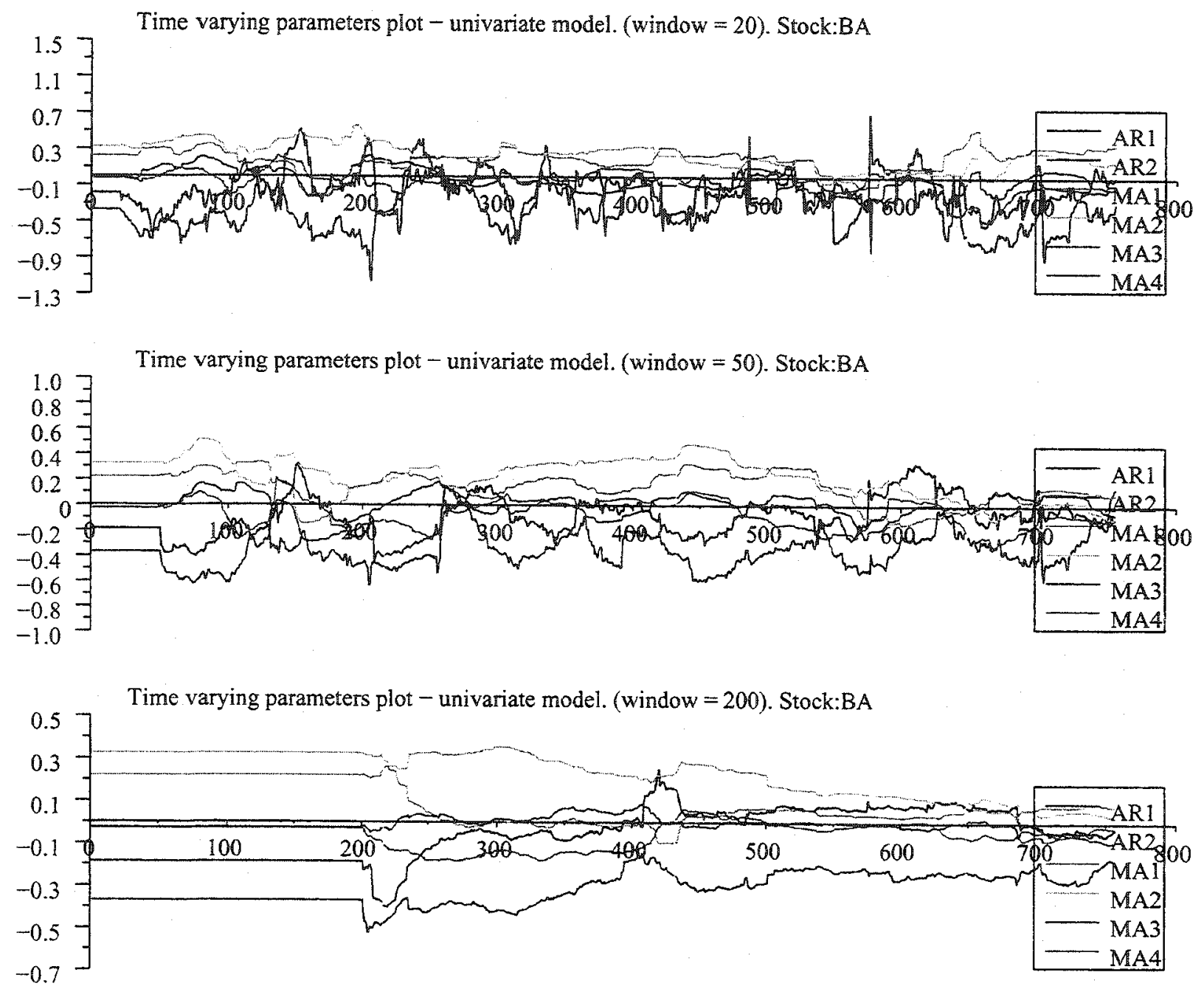

Figure H.11: Time varying parameter univariate model: parameter evolution over time for selected estimation window sizes. From top to bottom, window sizes are respectively, 20, 50 and 200 measurements. Stock: BA 

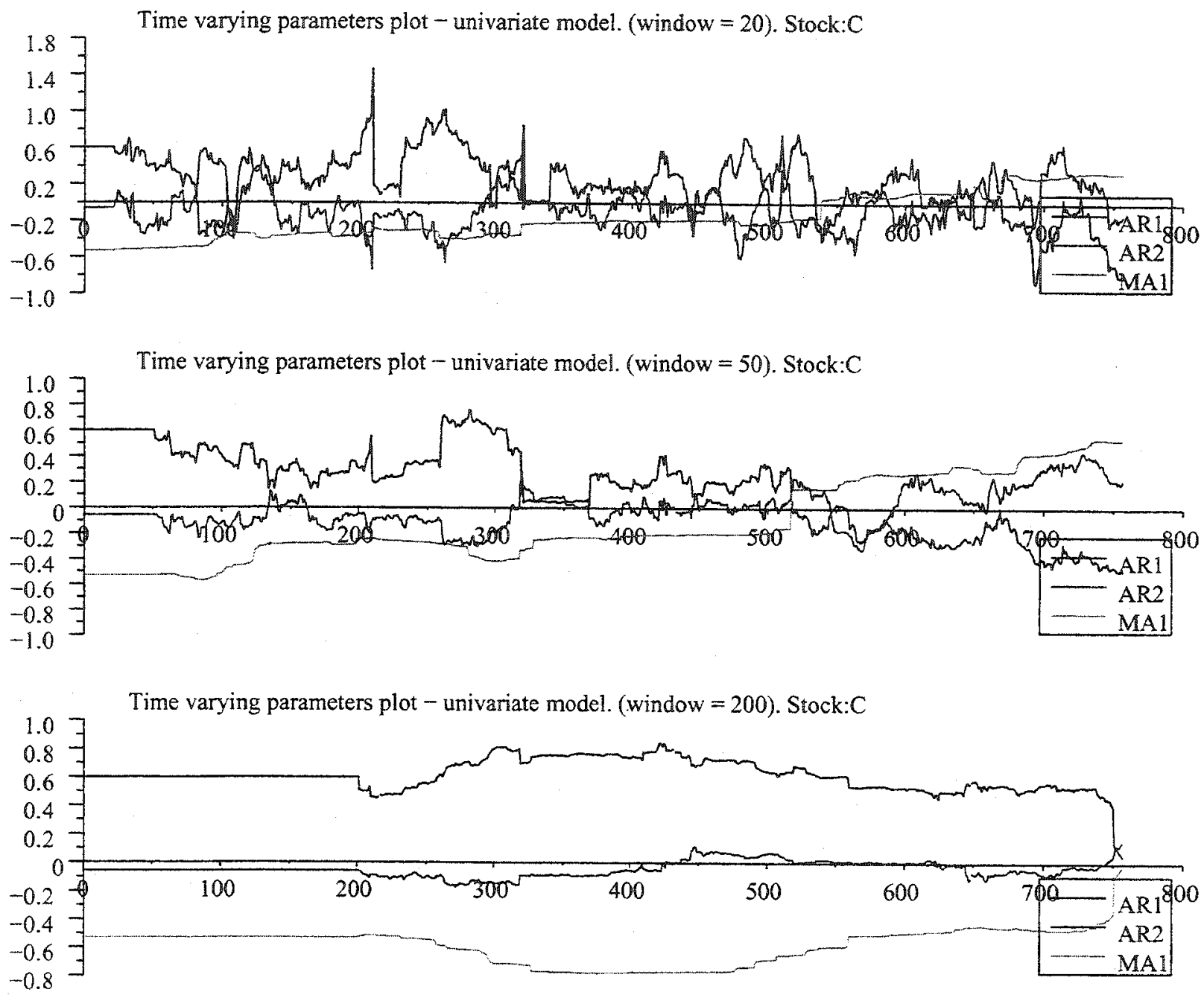

Figure H.12: Time varying parameter univariate model: parameter evolution over time for selected estimation window sizes. From top to bottom, window sizes are respectively, 20, 50 and 200 measurements. Stock: C 

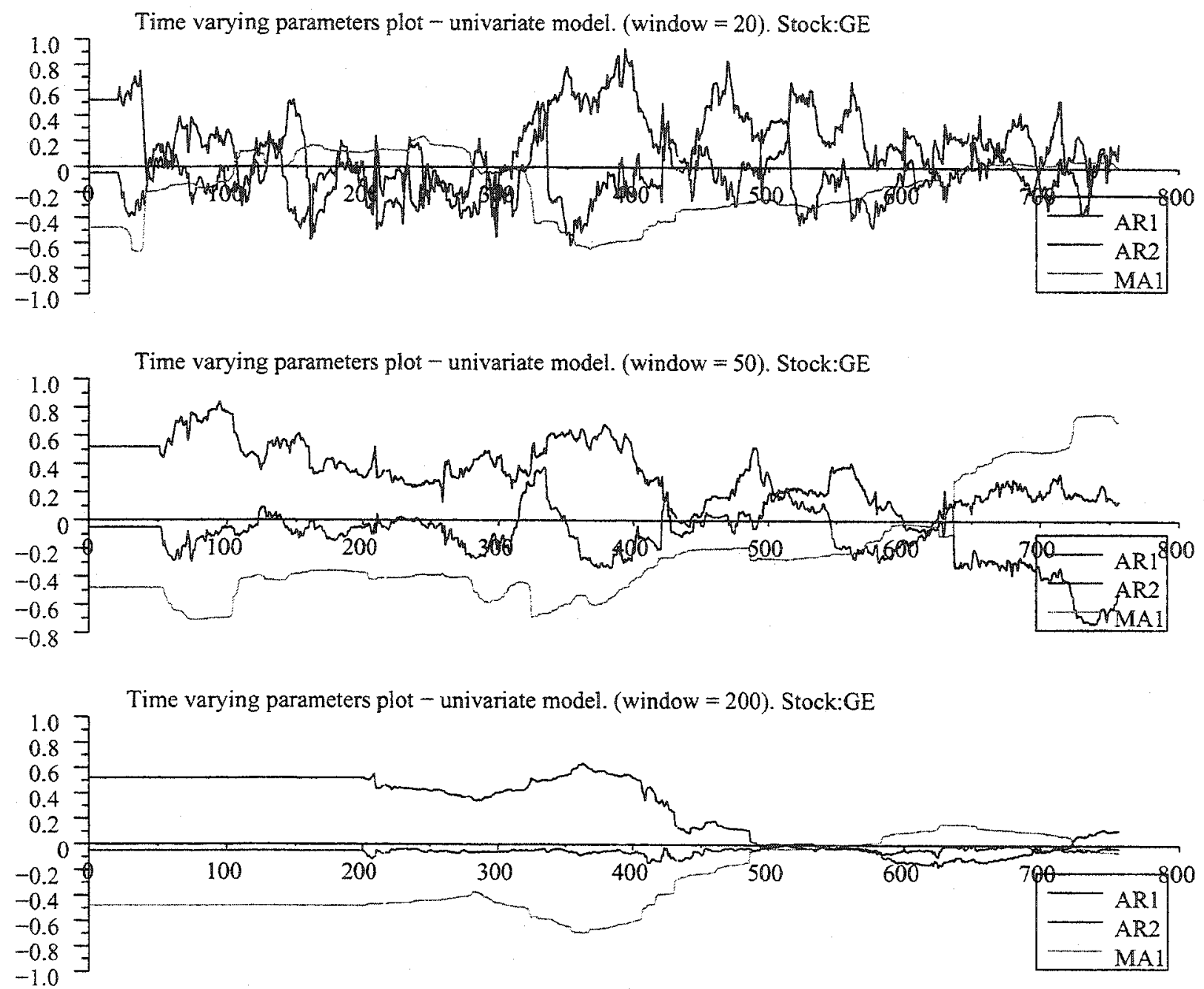

Figure H.13: Time varying parameter univariate model: parameter evolution over time for selected estimation window sizes. From top to bottom, window sizes are respectively, 20, 50 and 200 measurements. Stock: GE 

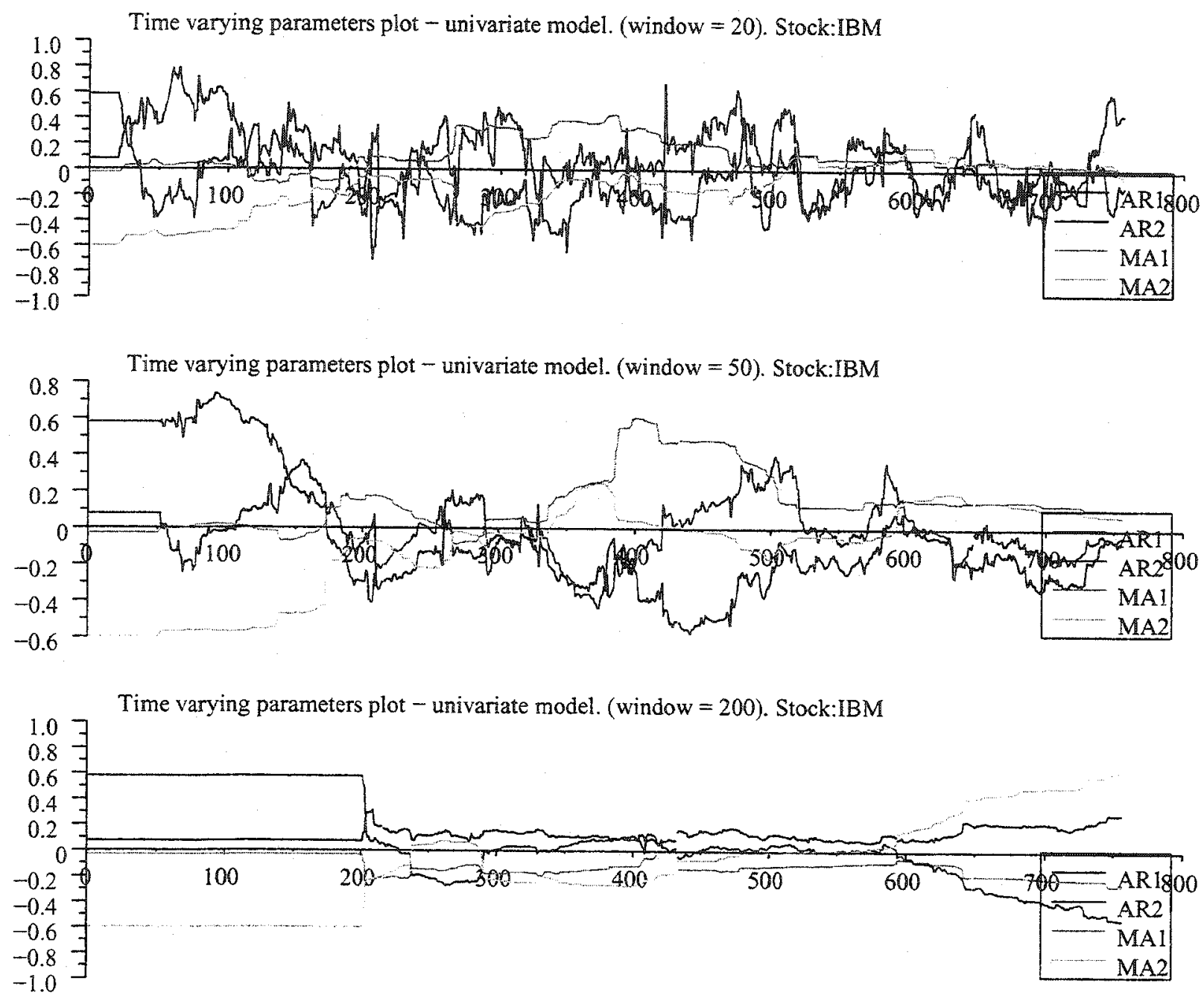

Figure H.14: Time varying parameter univariate model: parameter evolution over time for selected estimation window sizes. From top to bottom, window sizes are respectively, 20, 50 and 200 measurements. Stock: IBM 

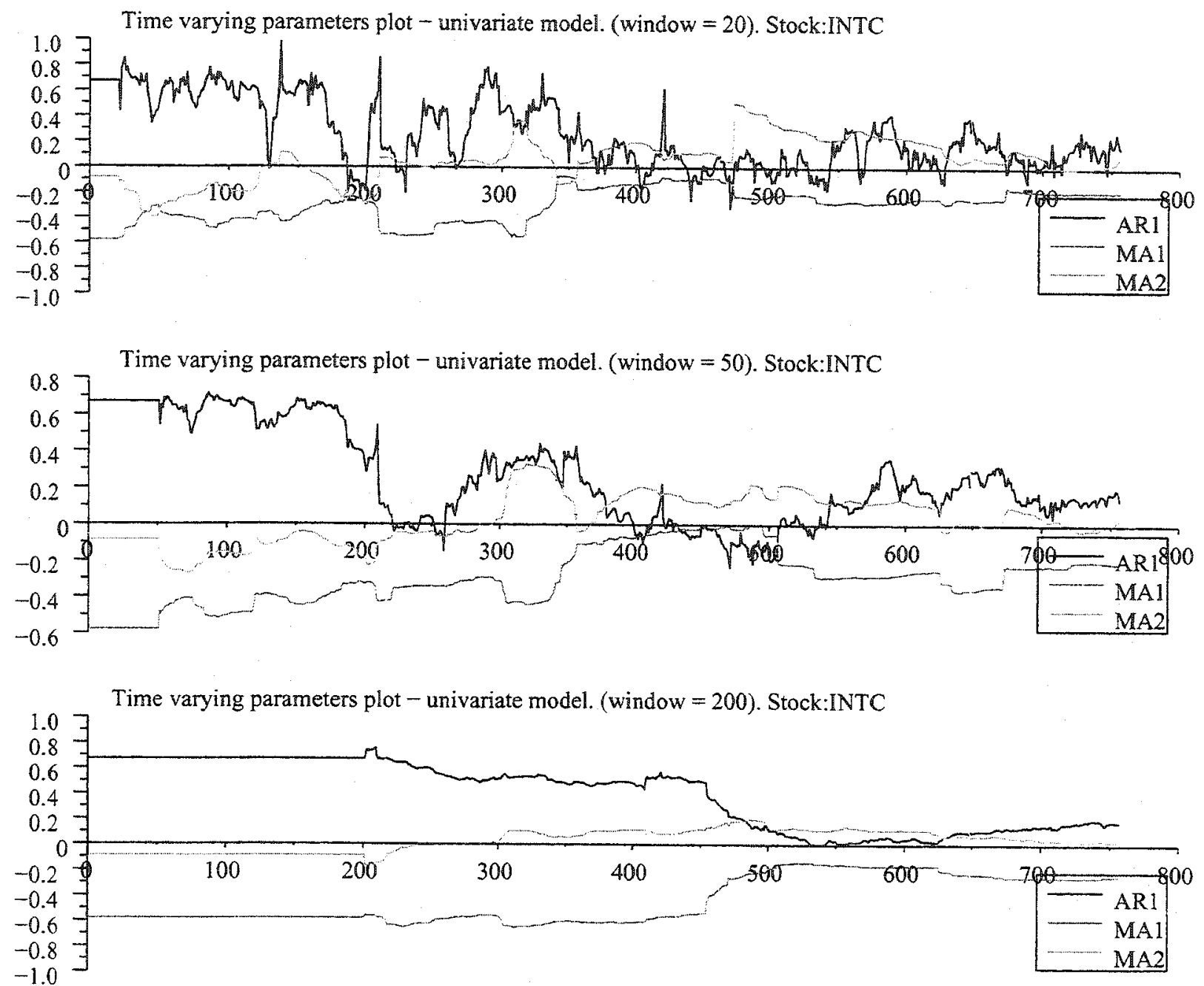

Figure H.15: Time varying parameter univariate model: parameter evolution over time for selected estimation window sizes. From top to bottom, window sizes are respectively, 20, 50 and 200 measurements. Stock: INTC 

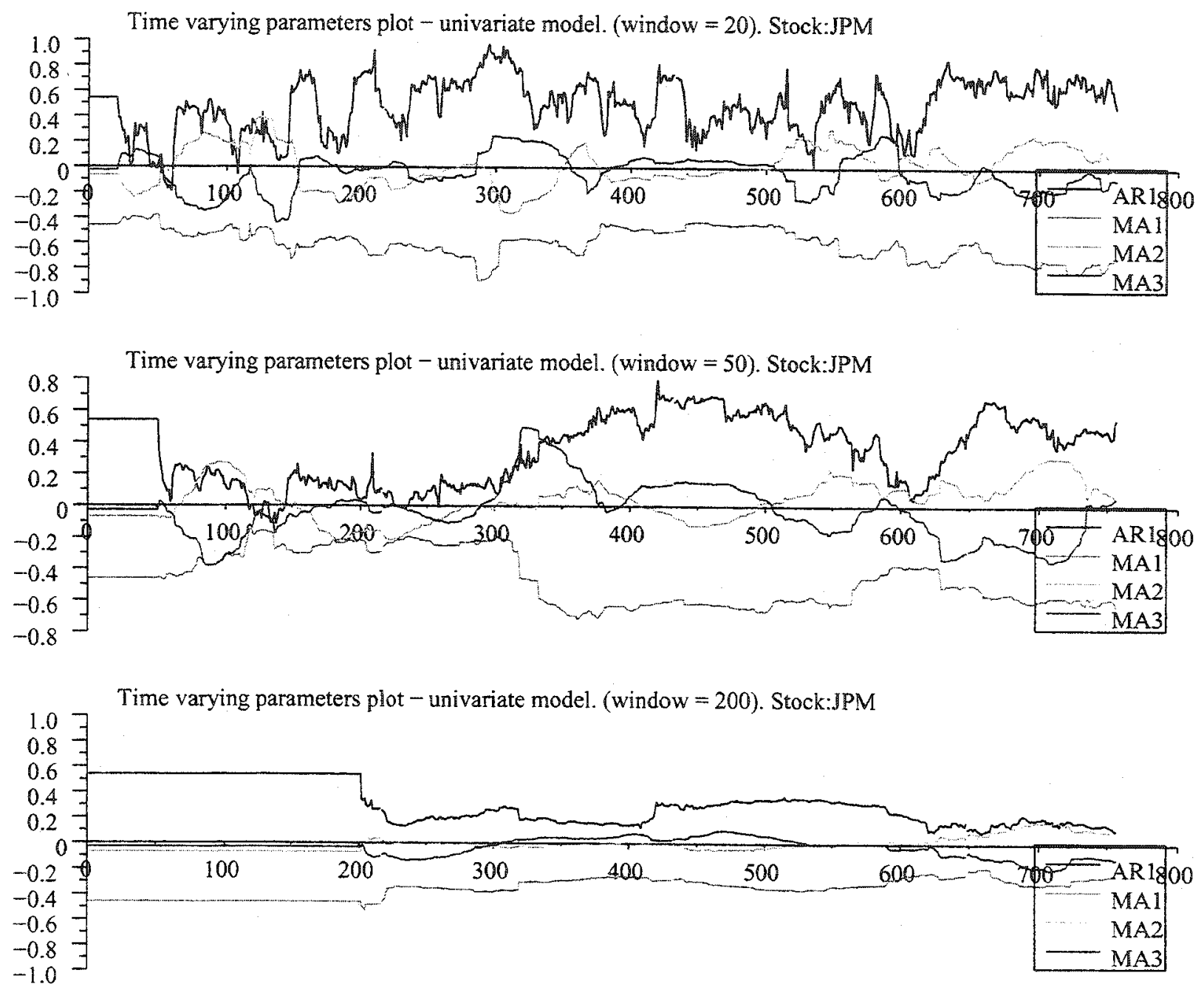

Figure H.16: Time varying parameter univariate model: parameter evolution over time for selected estimation window sizes. From top to bottom, window sizes are respectively, 20, 50 and 200 measurements. Stock: JPM 

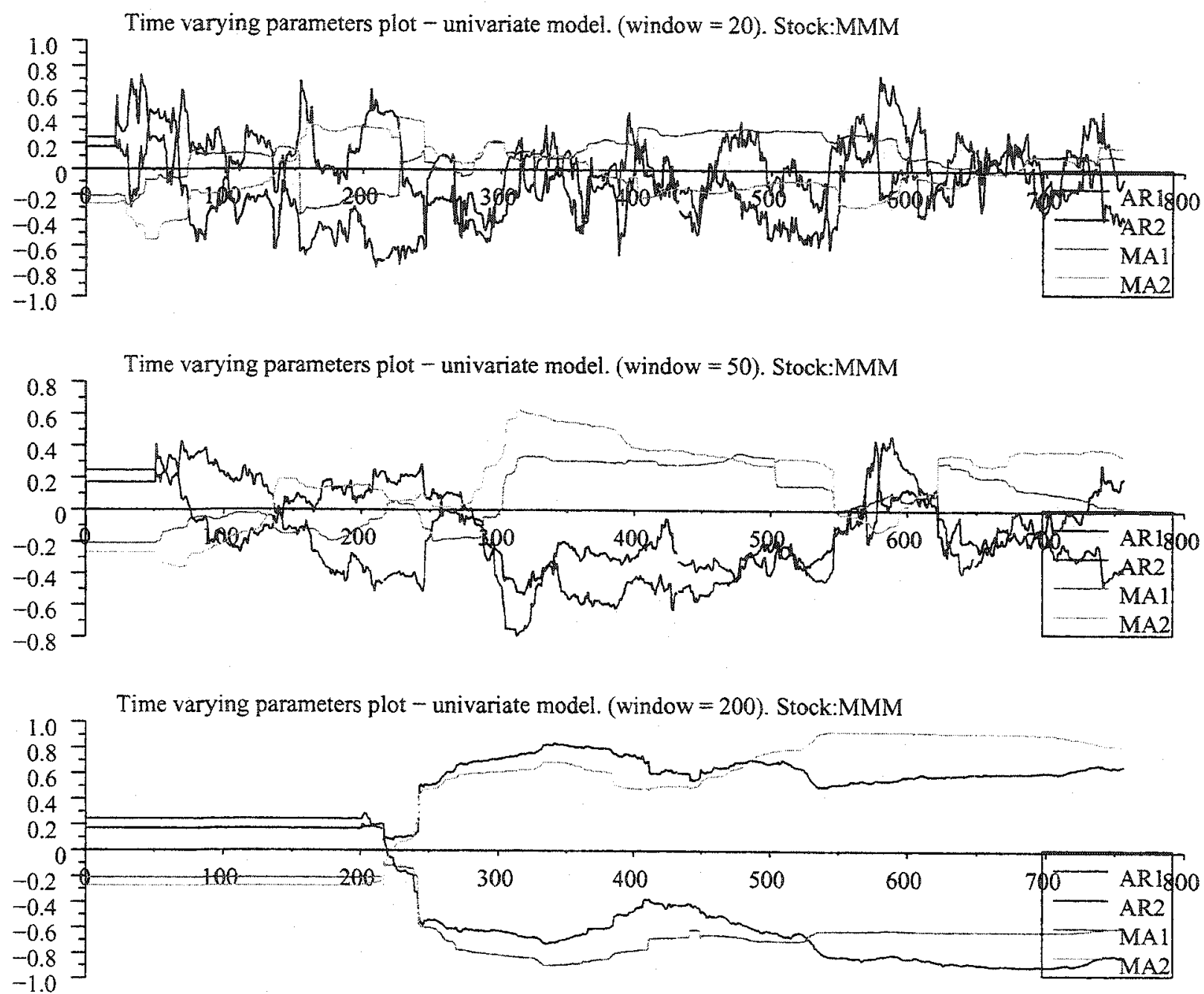

Figure H.17: Time varying parameter univariate model: parameter evolution over time for selected estimation window sizes. From top to bottom, window sizes are respectively, 20, 50 and 200 measurements. Stock: MMM 

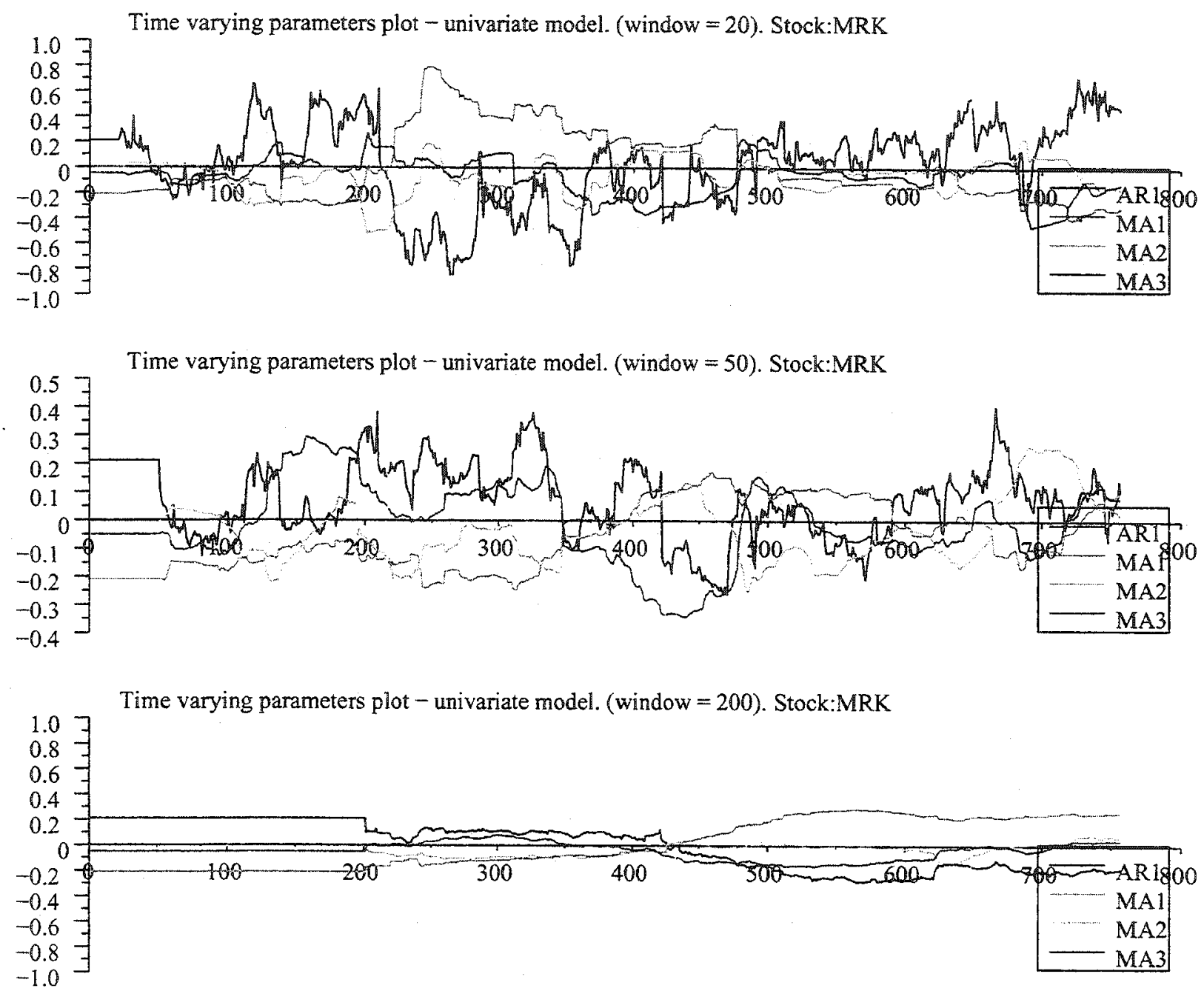

Figure H.18: Time varying parameter univariate model: parameter evolution over time for selected estimation window sizes. From top to bottom, window sizes are respectively, 20, 50 and 200 measurements. Stock: MRK 

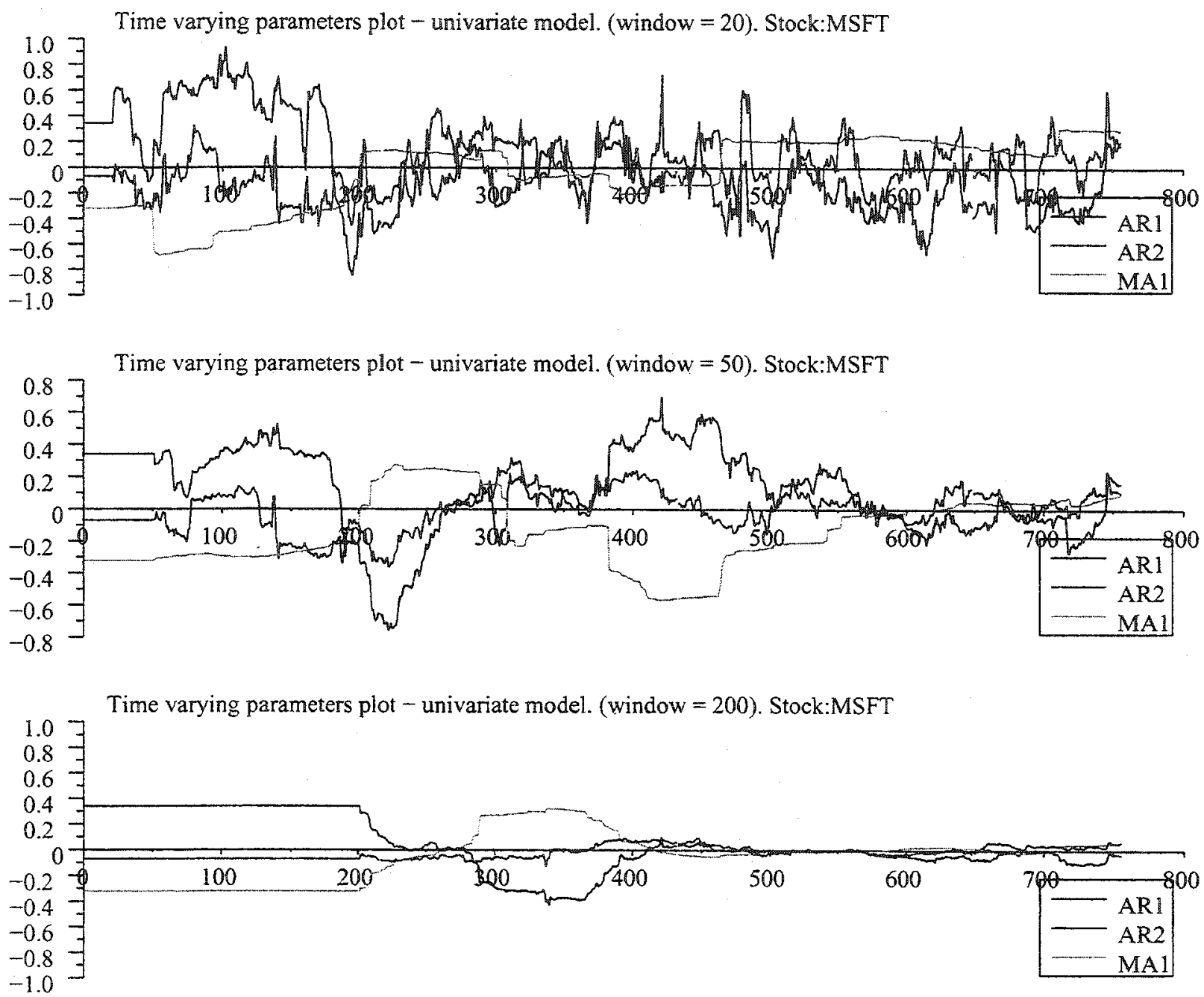

Figure H.19: Time varying parameter univariate model: parameter evolution over time for selected estimation window sizes. From top to bottom, window sizes are respectively, 20, 50 and 200 measurements. Stock: MSFT 

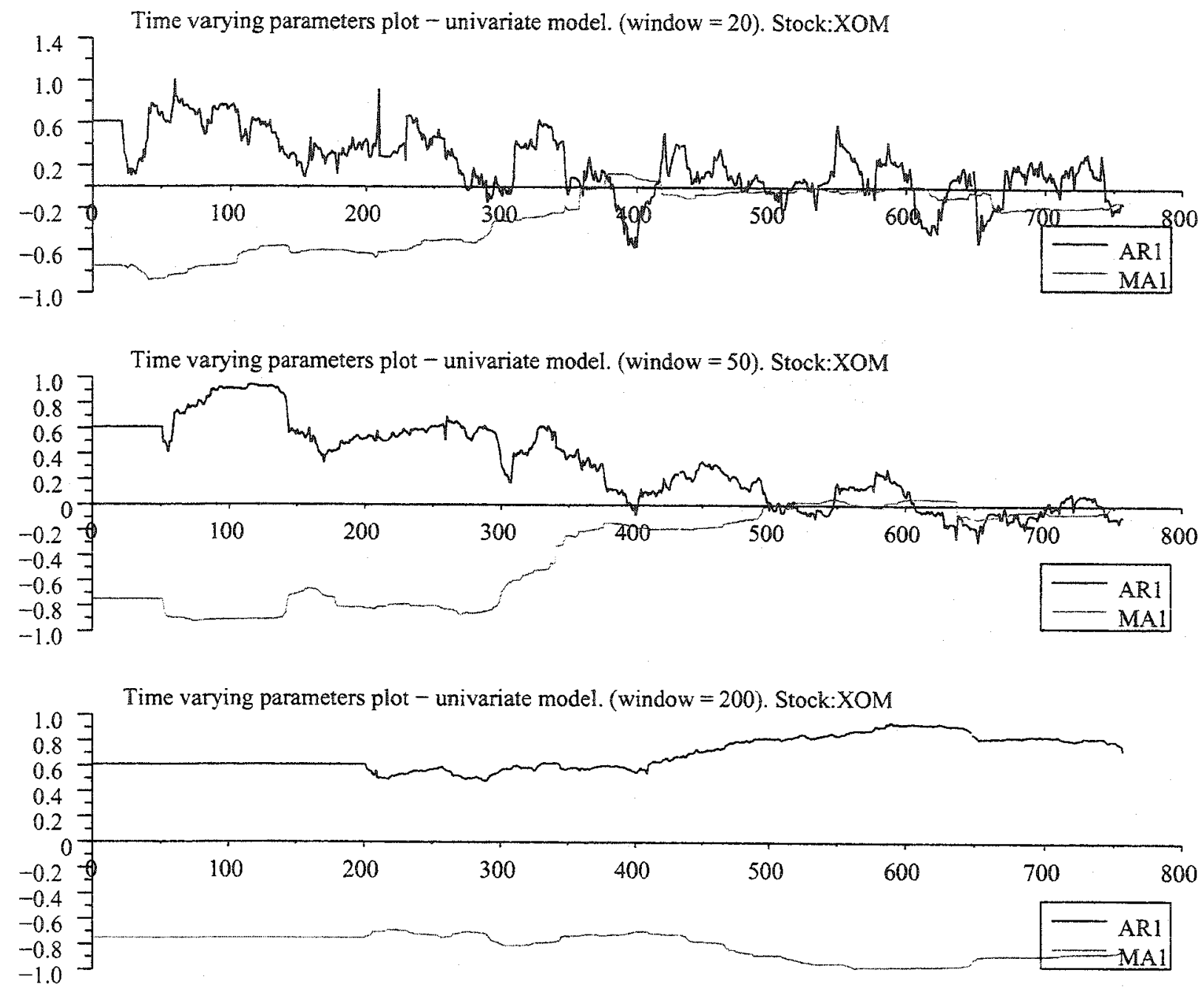

Figure H.20: Time varying parameter univariate model: parameter evolution over time for selected estimation window sizes. From top to bottom, window sizes are respectively, 20, 50 and 200 measurements. Stock: XOM 


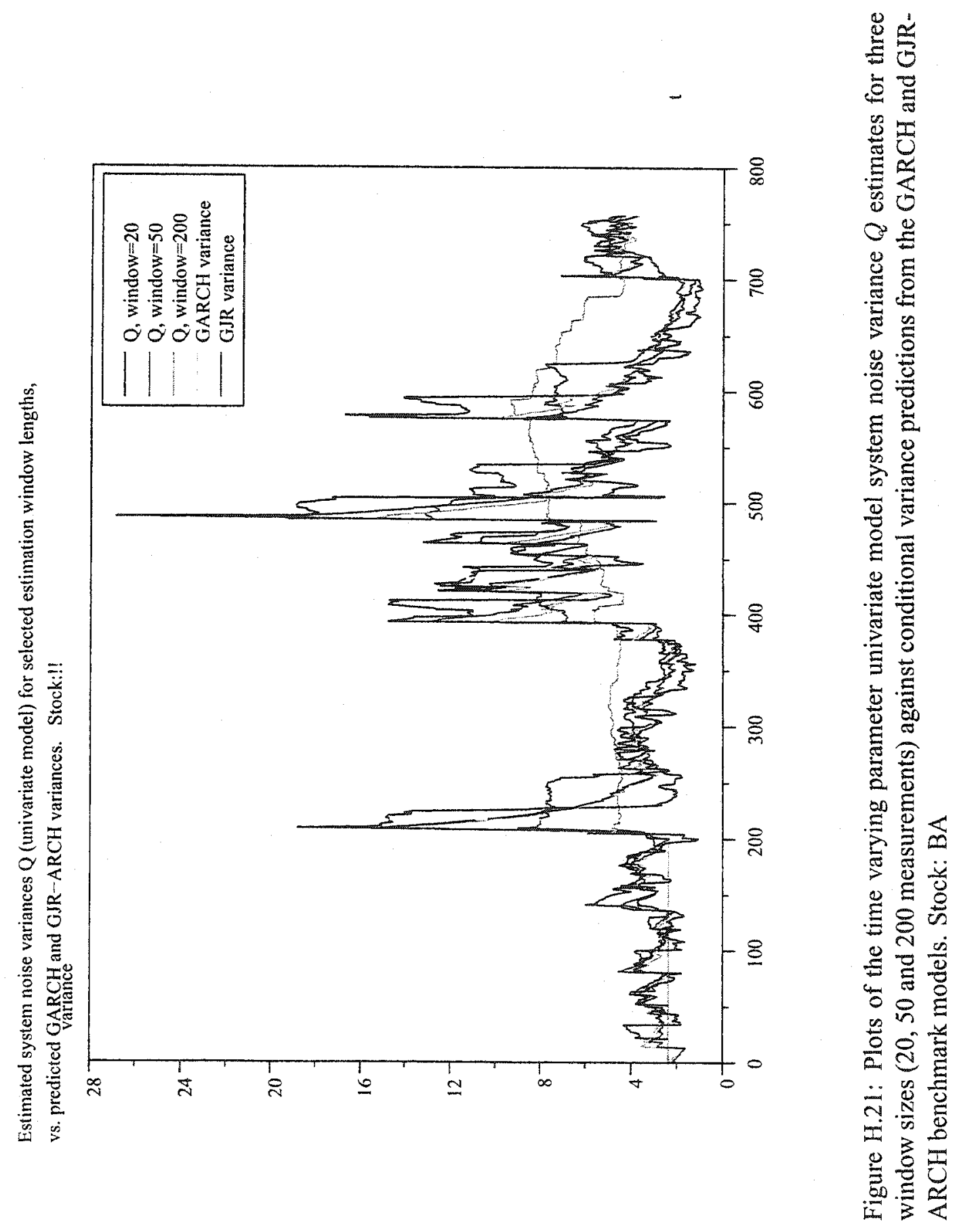




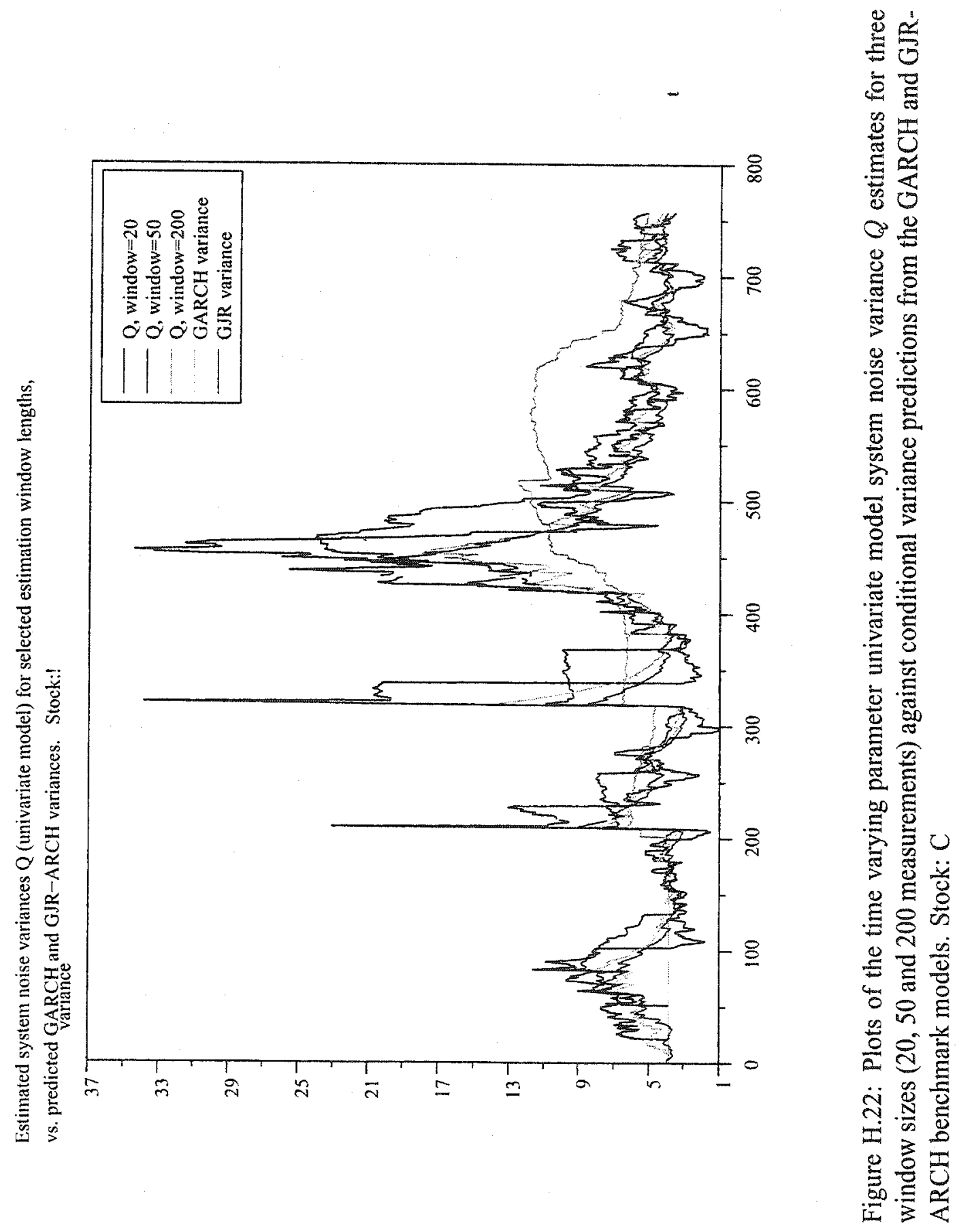




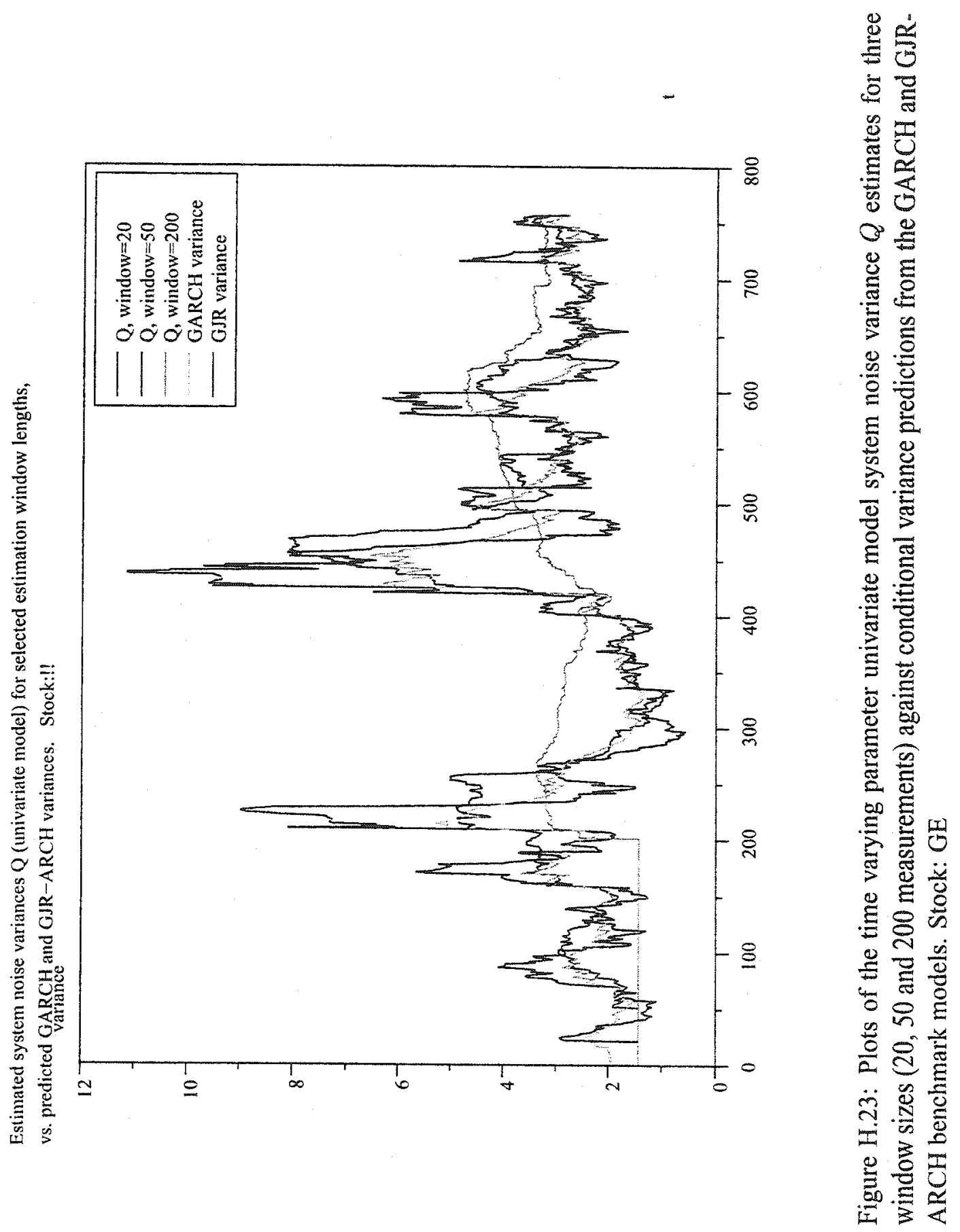




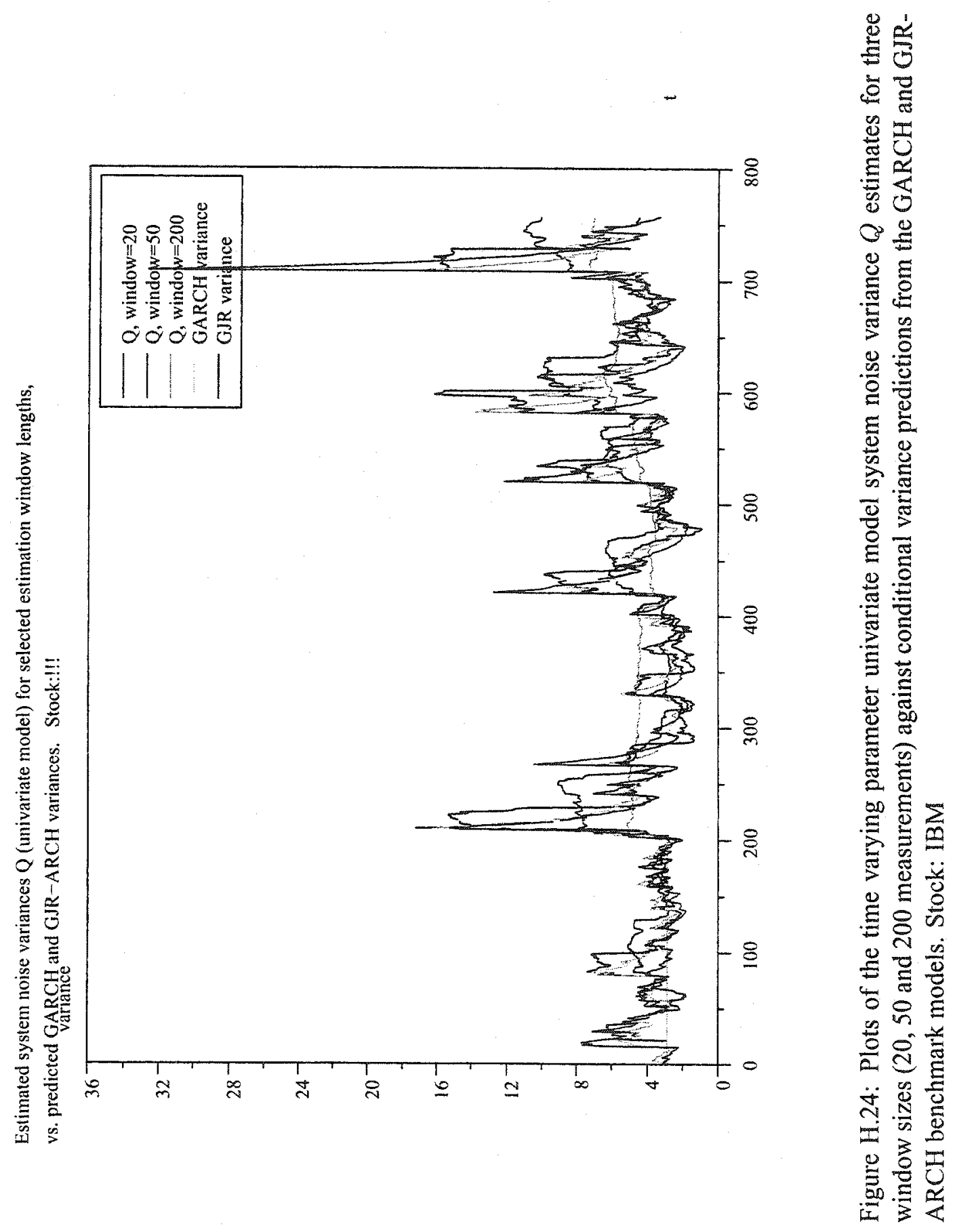




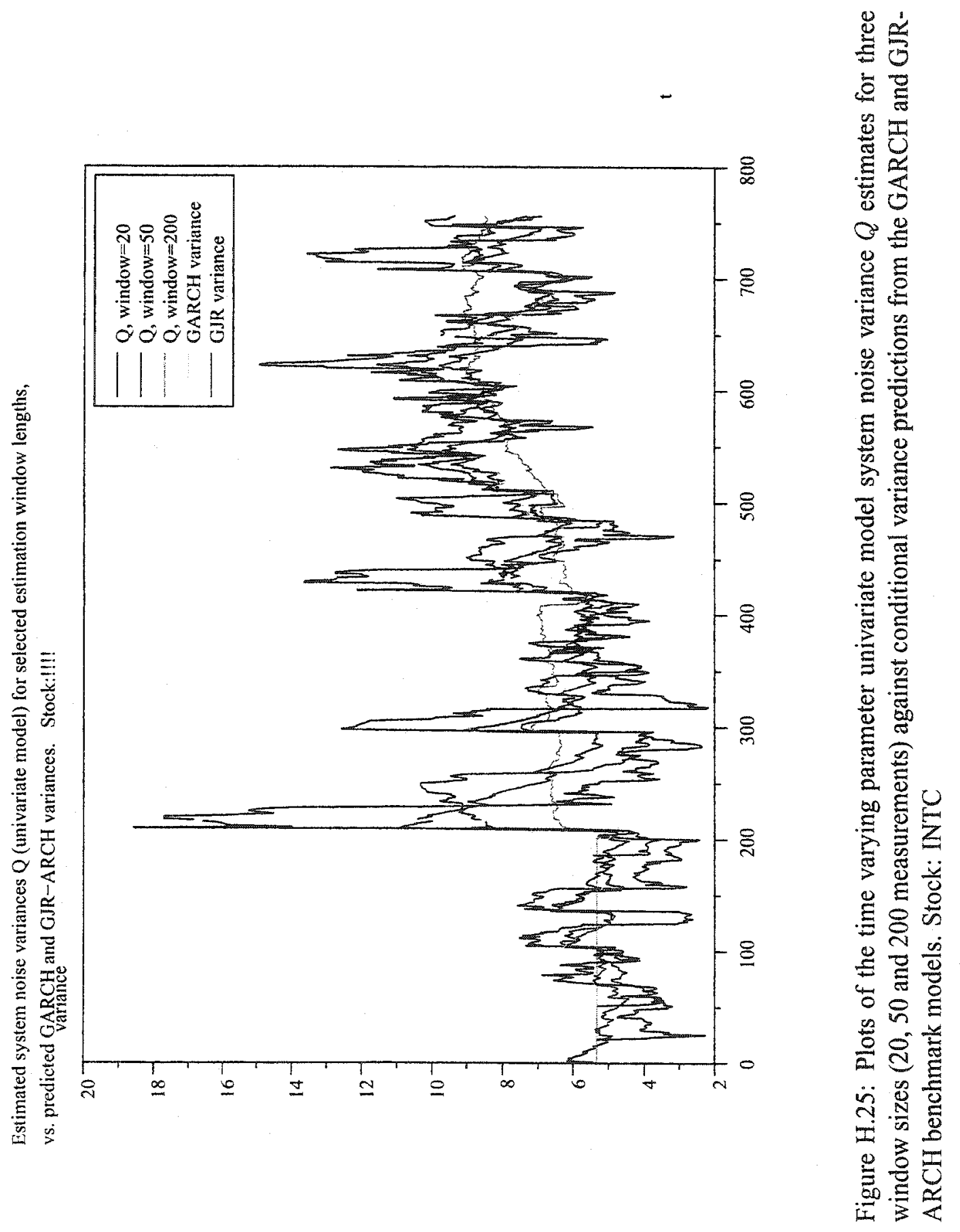




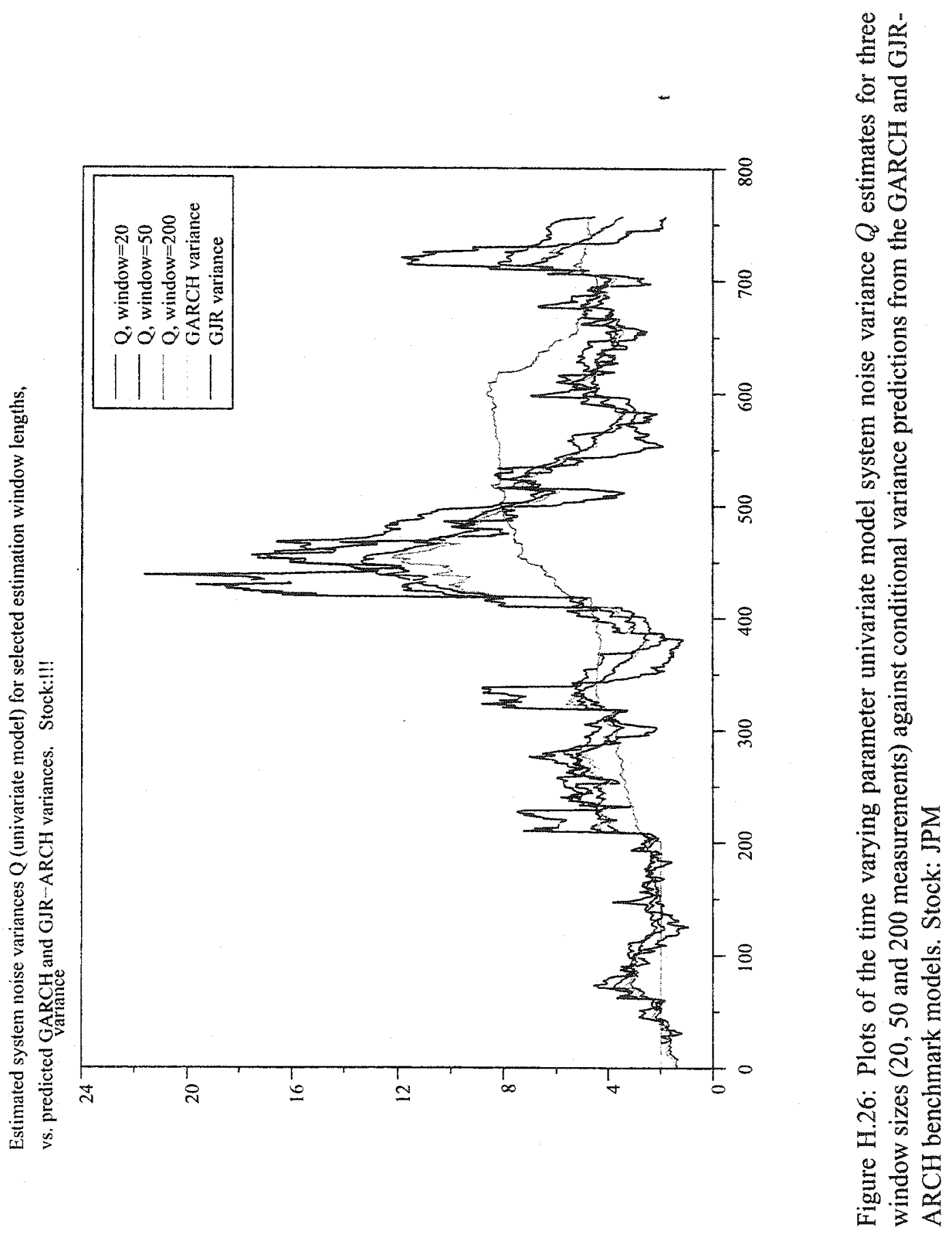




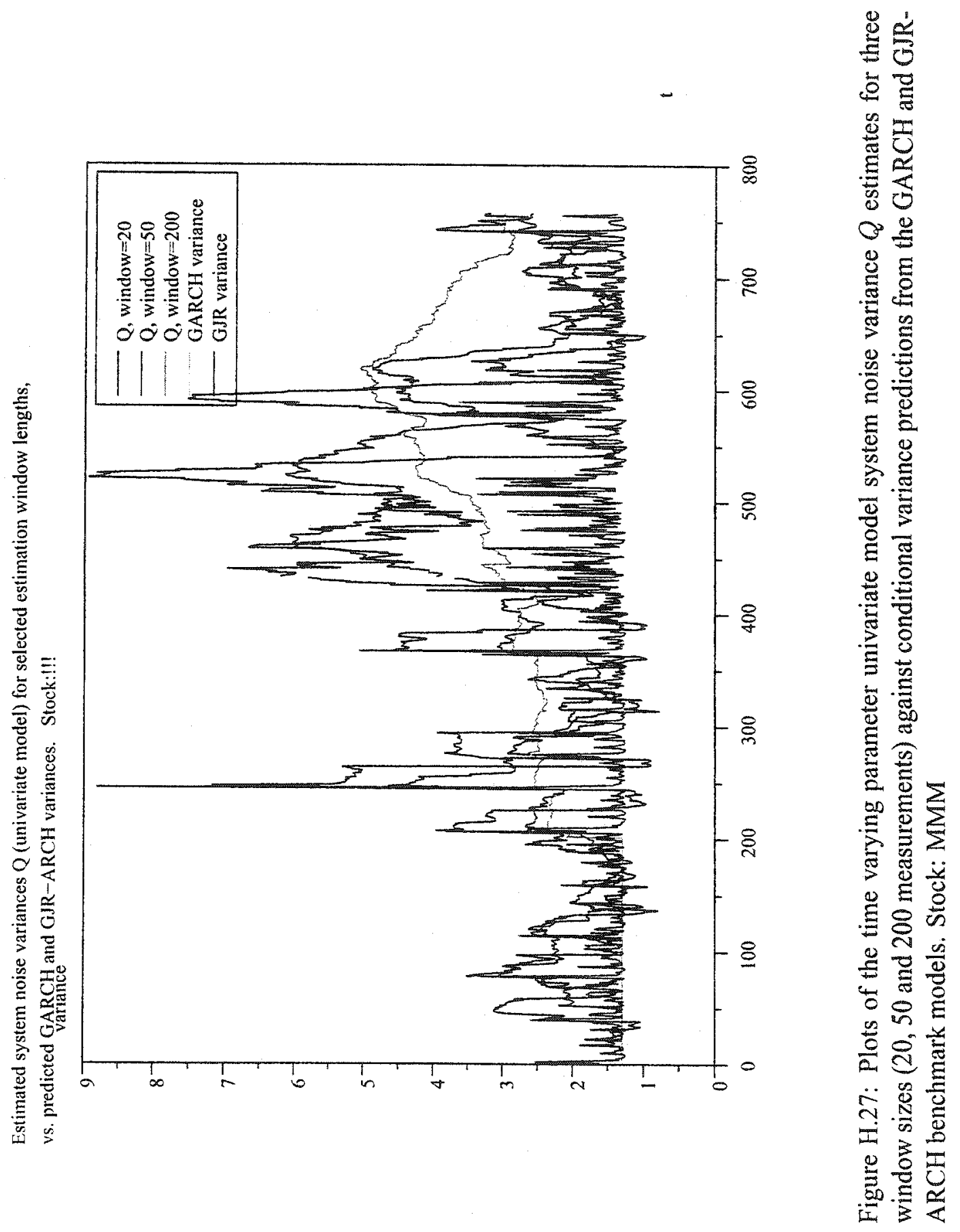




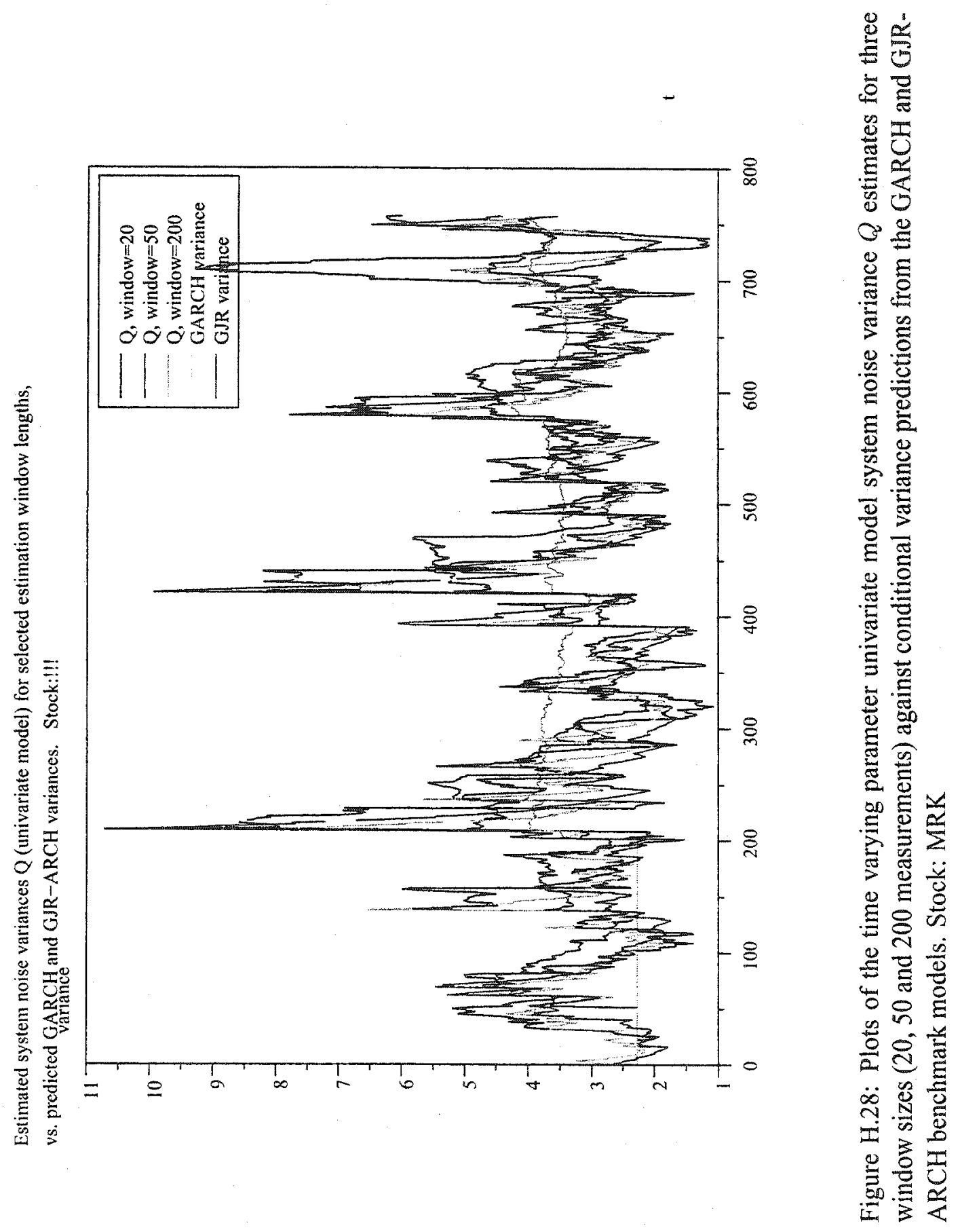




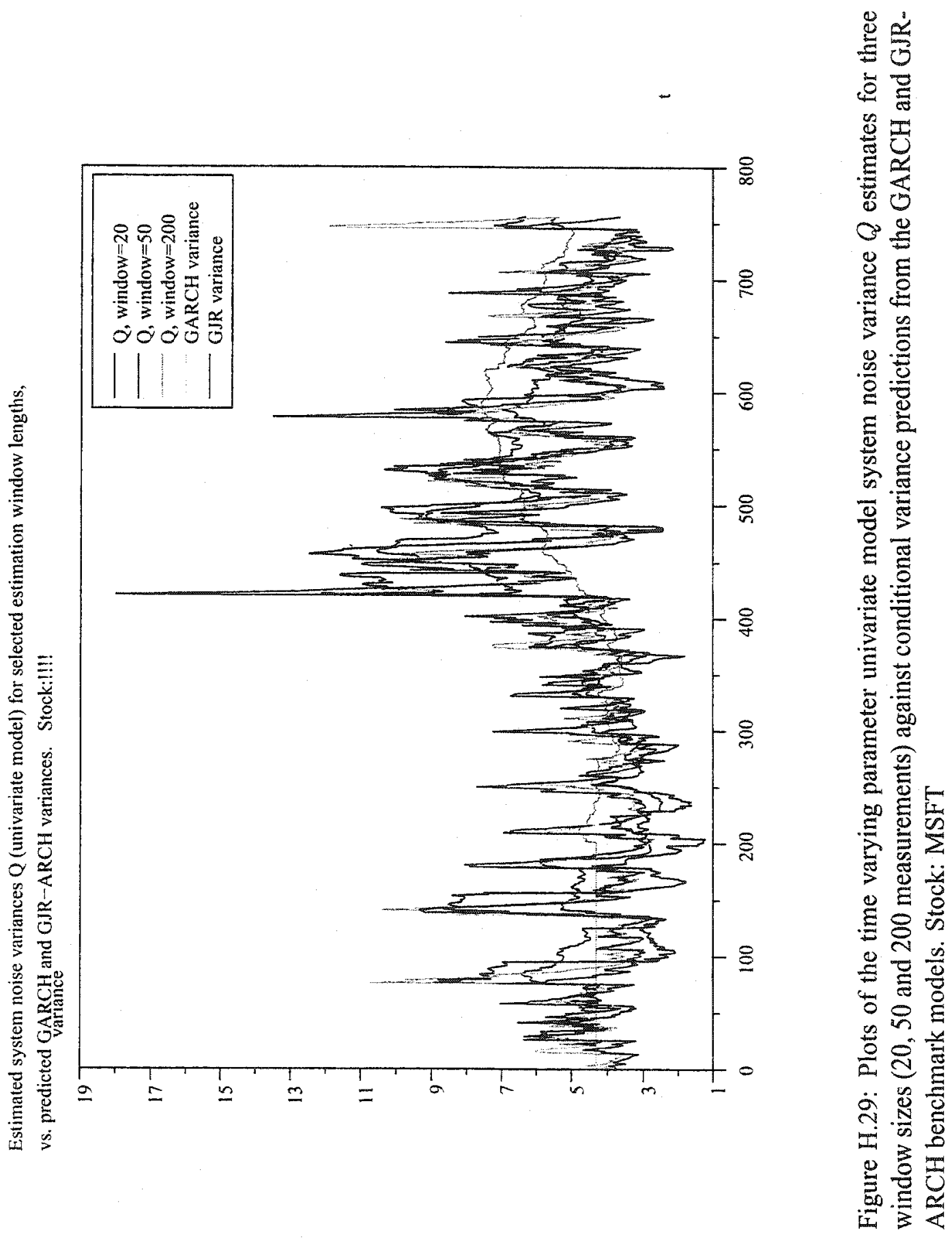




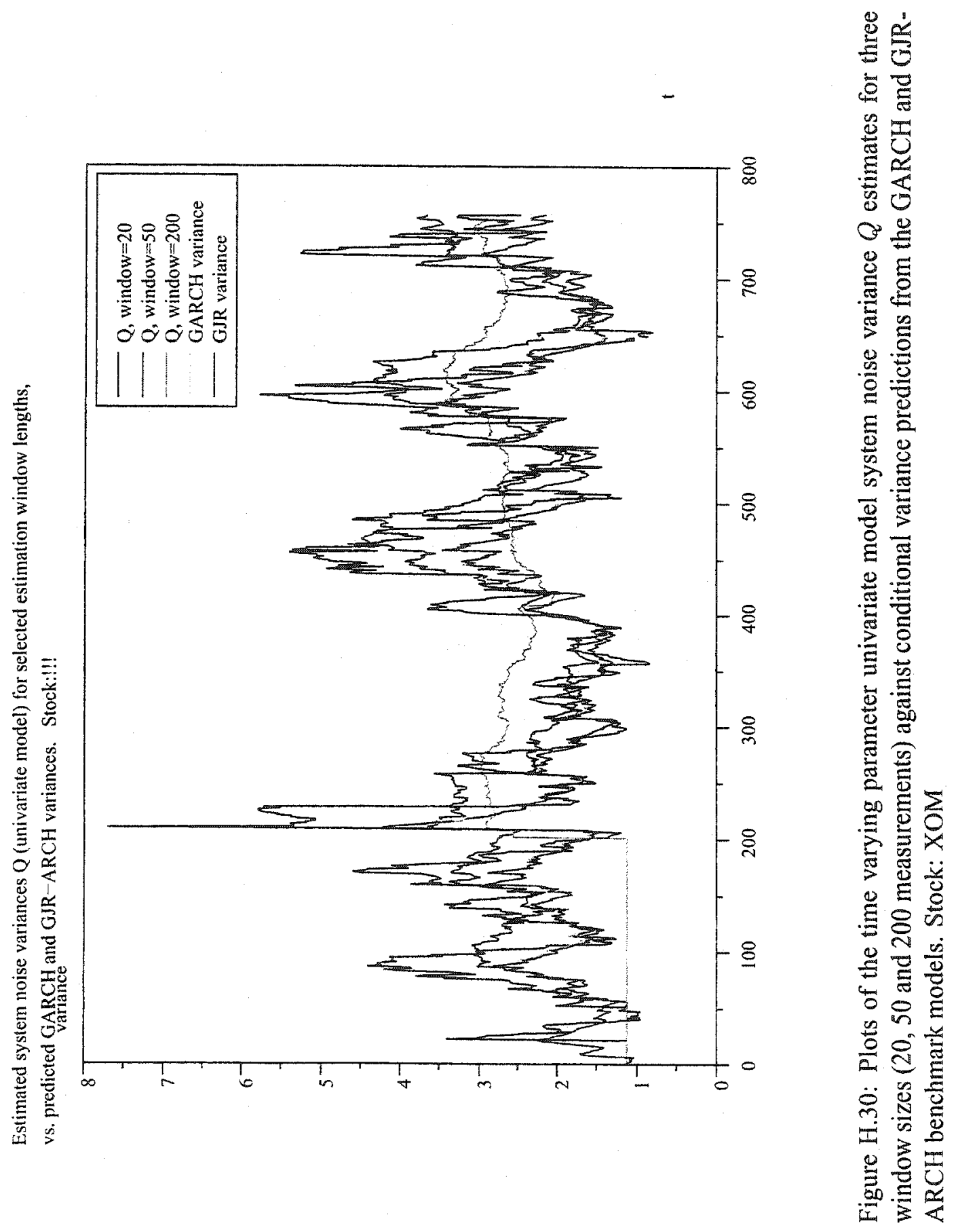




\section{Bibliography}

[Alexander, 1961] Alexander, S. S. (1961). Price movements in speculative markets. Industrian Management Review, 2(2):7-26. in Cootner ([Cootner, 1964]).

[Alexander, 1964] Alexander, S. S. (1964). Price movements in speculative markets. pages 338-372. in Cootner ([Cootner, 1964]).

[Allen and Karjalainen, 1999] Allen, F. and Karjalainen, R. (1999). Using genetic algorithms to find technical trading rules. Journal of Financial Economics, 51:245-275.

[Bachelier, 1900] Bachelier, L. (1900). Theory of Speculation. PhD thesis, Faculty of Sciences of the Academy of Paris. in Cootner ([Cootner, 1964]).

[Ball, 1988] Ball, R. (1988). What do we know about stock market 'efficiency'? In Guimaraes, R. M. C., Kingsman, B. G., and Taylor, S. J., editors, A Reappraisal of the Efficiency of the Financial Markets, volume 54 of NATO ASI Series F: Computer and Systems Science, pages 25-56. Springer-Verlag.

[Benartzi and Thaler, 1995] Benartzi, S. and Thaler, R. H. (1995). Myopic loss aversion and the equity premium puzzle. Quarterly Journal of Economics, pages $73-92$.

[Bikhchandani et al., 1992] Bikhchandani, S., Hirshleifer, D., and Welch, I. (1992). A theory of fads, fashion, custom, and cultural change and informational cascades. Journal of Political Economy, 100(5):992-1026.

[Black, 1976] Black, F. (1976). Studies of stock price volatility changes. In Proceedings of the American Statistical Association, volume Business and Economic Statistics, pages 177-181. 
[Blattberg and Gonedes, 1974] Blattberg, R. C. and Gonedes, N. J. (1974). A comparison of the stable and student distributions as statistical models of stock prices. Journal of Business, 47:244-280.

[Bollerslev, 1986] Bollerslev, T. (1986). Generalized autoregressive conditional heteroscedasticity. Journal of Econometrics, 31:307-327.

[Bollerslev et al., 1988] Bollerslev, T., Engle, R. F., and Woolridge, J. M. (1988). A capital asset pricing model with time varying covariances. Journal of Political Economy, 96(1):116-131.

[Boness et al., 1974] Boness, A. J., Chen, A. H., and Jatusipitak, S. (1974). Investigations of nonstationarity in prices. Journal of Business, 47:518-537.

[Breen et al., 1989] Breen, W., Glosten, L. R., and Jagannathan, R. (1989). Economic significance of predictable variations in stock index returns. Journal of Finance, 44(5):1177-1189.

[Brock et al., 1992] Brock, W., Lakonishok, J., and LeBaron, B. (1992). Simple technical trading rules and the stochastic properties of stock returns. Journal of Finance, 47(5):1731-1764.

[Brooks et al., 2001] Brooks, C., Burke, S. P., and Persand, G. (2001). Benchmarks and the accuracy of garch model estimation. International Journal of Forecasting, 17(1):45-56.

[Brown et al., 1998] Brown, S. J., Goetzmann, W. N., and Kumar, A. (1998). The dow theory: William peter hamilton's track record reconsidered. Journal of Finance, 53.

[Campbell, 1987] Campbell, J. Y. (1987). Stock returns and the term structure. Journal of Financial Economics, 18:373-399.

[Cergrene, 2001] Cergrene, S. G. I. M. P. (2001). Scilab software. http://wwwrocq.inria.fr/scilab/.

[Chan et al., 1998] Chan, L. K. C., Kacerski, J., and Lakonishok, J. (1998). The risk and return from factors. Journal of Financial and Quantitative Analysis, 33(2):159-188.

[Conrad and Kaul, 1988] Conrad, J. and Kaul, G. (1988). Time variation in expected returns. Journal of Business, 61(4):409-425. 
[Cootner, 1964] Cootner, P. H. (1964). The Random character of stock market prices. The MIT Press.

[Copeland and Mayers, 1982] Copeland, T. E. and Mayers, D. (1982). The value line enigma (1965-1978): A case study of performance evaluation issues. Journal of Financial Economics, pages 289-321.

[Copeland and Weston, 1992] Copeland, T. E. and Weston, J. F. (1992). Financial Theory and Corporate Policy. Addison-Wesley Publishing Company, third edition.

[Cowles, 1960] Cowles, A. (1960). A revision of previous conclusions regarding stock price behaviour. Econometrica, 28(4):909-915. in Cootner ([Cootner, 1964]).

[Cutler et al., 1989] Cutler, D. M., Poterba, J. M., and Summers, L. H. (1989). What moves stock prices? Journal of Portfolio Management, pages 4-12.

[Cutler et al., 1991] Cutler, D. M., Poterba, J. M., and Summers, L. H. (1991). Speculative dynamics. The Review of Economics and Statistics, 58:529-546.

[Daniel and Titman, 1999] Daniel, K. and Titman, S. (1999). Market efficiency in an irrational world. Financial Analysts Journal.

[Daniel et al., 1998] Daniel, K., Hirshleifer, D., and Subrahmanyam, A. (1998). Investor psychology and security market under and overreactions. Journal of Finance, 52(6):1839-1886.

[DeBondt and Thaler, 1985] DeBondt, W. F. M. and Thaler, R. (1985). Does the stock market overreact? Journal of Finance, 40(3):793-808.

[Doornik, 1999] Doornik, J. A. (1999). Object-Oriented Matrix Programming Using $O x$, 3rd ed. Timberlake Consultants Press and Oxford. www.nuff.ox.ac.uk/Users/Doornik.

[Engle, 1982] Engle, R. F. (1982). Autoregressive conditionasl heteroscedasticity with estimates of the variance of united kingdom inflation. Econometrica, 50(4):987-1006.

[Engle et al., 1987] Engle, R. F., Lilien, D. M., and Robins, R. P. (1987). Estimating time varying risk premia in the term structure: the arch-m model. Econometrica, 55(2):391-407. 
[Engle and Ng, 1993] Engle, R. F. and Ng, V. K. (1993). Measuring and testing the impact of news on volatility. Journal of Finance, 48(5):1749-1778.

[Fama, 1965] Fama, E. F. (1965). The behaviour of stock market prices. Journal of Finance, 38(1):34-105.

[Fama, 1970] Fama, E. F. (1970). Efficient capital markets: A review of theory and empirical work. Joumal of Finance, 25:383-417.

[Fama, 1991] Fama, E. F. (1991). Efficient capital markets: 2. Journal of Finance, 46(5).

[Fama and Blume, 1966] Fama, E. F. and Blume, M. E. (1966). Filter rules and stock market trading. Journal of Business, 39:226.

[Fama et al., 1969] Fama, E. F., Fisher, L., Jensen, M., and Roll, R. (1969). The adjustment of stock prices to new information. International Economic Review, 10:1-21.

[Fama and French, 1988a] Fama, E. F. and French, K. R. (1988a). Permanent and temporary components of stock prices. Journal of Political Economy, $96(2): 246$.

[Fama and French, 1988b] Fama, E. F. and French, K. R. (1988b). Permanent and temporary components of stock prices. Journal of Political Economy, $96(2): 246-273$.

[Fama and Roll, 1968] Fama, E. F. and Roll, R. (1968). Some properties of symmetric stable distributions. Journal of the American Statistical Association, 63:817-836.

[Fama and Roll, 1971] Fama, E. F. and Roll, R. (1971). Parameter estimates for symmetric stable distributions. Journal of the American Statistical Association, 66:331-338.

[Fama and Schwert, 1977] Fama, E. F. and Schwert, G. W. (1977). Asset returns and inflation. Journal of Econometrics, 5:115-146.

[French, 1980] French, K. (1980). Stock returns and the weekend effect. Journal of Financial Economics, 58. 
[French et al., 1987] French, K. R., Schwert, G. W., and Stambaugh, R. F. (1987). Expected stock returns and volatility. Journal of Financial Economics, 19:329.

[Glosten et al., 1993] Glosten, L. R., Jagannathan, R., and Runkle, D. (1993). On the relation between the expected value and the volatility of the nominal excess return on stocks. Journal of Finance, 48:1779-1802.

[Harrison and Stevens, 1976] Harrison, P. J. and Stevens, C. F. (1976). Bayesian forecasting. Journal of the Royal Statistical Society, B, 38:205-247.

[Harvey, 1989] Harvey, A. C. (1989). Forecasting structural time series models and the Kalman filter. CUP.

[Hong et al., 2000] Hong, H., Lim, T., and Stein, J. C. (2000). Bad news travels slowly: Size, analyst coverage and the profitability of momentum strategies. Journal of Finance, 55(1):265-295.

[Hong and Stein, 1999] Hong, H. and Stein, J. C. (1999). A unified theory of underreaction, momentum trading and overreaction in asset markets. Journal of Finance, 54(6):2143-2184.

[Hsu et a1., 1974] Hsu, D.-A., Miller, R. B., and Wichern, D. W. (1974). On the stable paretian behaviour of stock market prices. Journal of the American Statistical Association, 69(345):108-113.

[Jegadeesh, 1990] Jegadeesh, N. (1990). Evidence of predictable behaviour of security returns. Journal of Finance, 45(3):881-898.

[Jensen, 1978] Jensen, M. C. (1978). Some anomalous evidence regarding market efficiency. Joumal of Financial Economics, 6:95-101.

[Keim and Stambaugh, 1986] Keim, D. B. and Stambaugh, R. F. (1986). Predicting returns in the stock and bond markets. Journal of Financial Economics, $17: 357-390$.

[Kendall, 1953] Kendall, M. G. (1953). The analysis of economic time-series part 1: Prices. Joumal of the Royal Statistical Society, A, 96:11-25. in Cootner ([Cootner, 1964]). 
[Kim et al., 1991] Kim, M. J., Nelson, C. R., and Startz, R. (1991). Mean reversion in stock prices? a reappraisal of the empirical evidence. The Review of Economics and Statistics, 58:515-528.

[Kon, 1984] Kon, S. J. (1984). Models of stock returns - a comparison. Journal of Finance, 39(1):147.

[Laurent and Peters, 2002] Laurent, S. and Peters, J. P. (2002). G@rch 2.2: An ox package for estimating and forecasting various arch models. Journal of Economic Surveys, 16(3):447-484.

[LeRoy, 1989] LeRoy, S. F. (1989). Efficient capital markets and martingales. Journal of Economic Literature, 27:1583-1621.

[Lo and MacKinlay, 1988] Lo, A. W. and MacKinlay, A. C. (1988). Stock market prices do not follow random walks: Evidence from a simple specification test. Review of Financial Studies, 1(1):41-66.

[Lo and Mackinlay, 1990] Lo, A. W. and Mackinlay, A. C. (1990). When are contrarian profits due to stock market overreaction? Review of Financial Studies, 3(2):175-205.

[Mandelbrot, 1963] Mandelbrot, B. (1963). The variation of certain speculative prices. Journal of Business, 34(4):394-419. in Cootner ([Cootner, 1964]).

[Mandelbrot, 1967] Mandelbrot, B. B. (1967). The variation of the price of cotton, wheat, and railroad stocks, and some financial rates. Journal of Business, $40: 393-413$.

[Maybeck, 1972] Maybeck, P. S. (1972). Combined state and parameter estimation for on-line applications. $\mathrm{PhD}$ thesis, MIT.

[Maybeck, 1979] Maybeck, P. S. (1979). Stochastic Models, Estimation, and Control, volume 1. Academic Press.

[Maybeck, 1981] Maybeck, P. S. (1981). Stochastic Models, Estimation, and Control, volume 2. Academic Press.

[Meinhold and Singpurwalla, 1983] Meinhold, R. J. and Singpurwalla, N. D. (1983). Understanding the kalman-filter. The American Statistician, 37:123127. 
[Neftci, 1991] Neftci, S. N. (1991). Naive trading rules in financial markets and wiener-kolmogorov prediction theory: a study of 'technical analysis'. Journal of Business, 64(4):549-571.

[Nelson, 1991] Nelson, D. B. (1991). Conditional heteroscedastisity in asset returns: A new approach. Econometrica, 59(2):347-370.

[Osborne, 1959] Osborne, M. F. M. (1959). Brownian motion in the stock market. Operations Research, 7:145-173. in Cootner ([Cootner, 1964]).

[Pesaran and Timmerman, 1992] Pesaran, M. H. and Timmerman, A. (1992). A simple nonparametric test of predictive performance. Joumal of Business and Economic Statistics, 10:461-465.

[Pesaran and Timmerman, 1994] Pesaran, M. H. and Timmerman, A. (1994). Forecasting stock returns: and examination of stock market trading in the presence of transaction costs. Journal of Forecasting, 13:335-337.

[Pesaran and Timmerman, 1995] Pesaran, M. H. and Timmerman, A. (1995). Predictability of stock returns: Robustness and economic significance. Journal of Finance, 50(4):1201-1228.

[Pesaran and Timmerman, 1999] Pesaran, M. H. and Timmerman, A. (1999). A recursive modelling approach to predicting uk stock returns. Working Paper.

[Poterba and Summers, 1988] Poterba, J. M. and Summers, L. H. (1988). Mean reversion in stock prices. Journal of Financial Economics, 22:27-59.

[Roll, 1988] Roll, R. (1988). $r^{2}$. Journal of Finance, 43(3).

[Rozeff, 1974] Rozeff, M. S. (1974). Money and stock prices. Journal of Financial Economics, 1:245.

[Samuelson, 1965] Samuelson, P. A. (1965). Proof that properly anticipated prices fuctuate randomly. Industrial Management Review, 6:41-50.

[Samuelson, 1973] Samuelson, P. A. (1973). Mathematics of speculative price. SIAM Review, 15(1):1-42.

[Shelton, 1967] Shelton, J. (1967). The value line contest: A test of the predictability of stock price changes. Journal of Business, pages 251-269. 
[Shiller, 1981] Shiller, R. J. (1981). Do stock prices move too much to be justified by subsequent changes in dividends? American Economic Review, pages 421436.

[Stickel, 1985] Stickel, S. (1985). The effect of value line investment survey rank changes on common stock prices. Journal of Financial Economics, pages 121 144.

[Sweeney, 1988] Sweeney, R. J. (1988). Some new filter rule tests: Methods and results. Journal of Financial and Quantitative Analysis, 23(3):285-300.

[Taussig, 1921] Taussig, F. W. (1921). Is market price indeterminate? Quarterly Journal of Economics, 35:394-411.

[Taylor, 1980] Taylor, S. J. (1980). Conjectured models for trends in financial prices, tests and forecasts. Journal of the Royal Statistical Society, A, 143:338362.

[Thaler, 1999] Thaler, R. H. (1999). The end of behavioural finance. Financial Analysts Journal.

[Theil, 1966] Theil, H. (1966). Applied Econometric Forecasting, volume 4. North-Holland Publishing Company.

[Working, 1934] Working, H. (1934). A random difference series for use in the analysis of time series. Journal of the American Statistical Association, 29:1124.

[Working, 1958] Working, H. (1958). A theory of anticipatory prices. American Economic Review, 38:189-199.

[Zhou, 1996] Zhou, C. (1996). Forecasting long and short horizon stock returns in a unified framework. Technical report, Federal Reserve Board. czhou@frb.gov. 